\title{
Optical Imaging
}

Citation for published version (APA):

Alves, F., Bode, J., Cimalla, P., Hilger, I., Hofmann, M., Jaedicke, V., Koch, E., Licha, K., Rademakers, T., Razansky, D., \& Van Zandvoort, M. A. M. J. (2017). Optical Imaging. In F. Kiessling, B. J. Pichler, \& P. Hauff (Eds.), Small Animal Imaging: Basics and Practical Guide (2 ed., pp. 403-490). Springer. https://doi.org/10.1007/978-3-319-42202-2_16

\section{Document status and date:}

Published: 23/05/2017

DOI:

10.1007/978-3-319-42202-2_16

Document Version:

Publisher's PDF, also known as Version of record

\section{Document license:}

Taverne

\section{Please check the document version of this publication:}

- A submitted manuscript is the version of the article upon submission and before peer-review. There can be important differences between the submitted version and the official published version of record.

People interested in the research are advised to contact the author for the final version of the publication, or visit the DOI to the publisher's website.

- The final author version and the galley proof are versions of the publication after peer review.

- The final published version features the final layout of the paper including the volume, issue and page numbers.

Link to publication

\footnotetext{
General rights rights.

- You may freely distribute the URL identifying the publication in the public portal. please follow below link for the End User Agreement:

www.umlib.nl/taverne-license

Take down policy

If you believe that this document breaches copyright please contact us at:

repository@maastrichtuniversity.nl

providing details and we will investigate your claim.
}

Copyright and moral rights for the publications made accessible in the public portal are retained by the authors and/or other copyright owners and it is a condition of accessing publications that users recognise and abide by the legal requirements associated with these

- Users may download and print one copy of any publication from the public portal for the purpose of private study or research.

- You may not further distribute the material or use it for any profit-making activity or commercial gain

If the publication is distributed under the terms of Article $25 \mathrm{fa}$ of the Dutch Copyright Act, indicated by the "Taverne" license above, 


\title{
Optical Imaging
}

\author{
Frauke Alves, Julia Bode, Peter Cimalla, \\ Ingrid Hilger, Martin Hofmann, Volker Jaedicke, \\ Edmund Koch, Kai Licha, Timo Rademakers, \\ Daniel Razansky, and Marc A.M.J. van Zandvoort
}

\section{Contents}

16.1 Fluorescence Imaging

16.1.1 Introduction

16.1.2 In Vivo Fluorescence Imaging Systems ....

16.1.3 Contrast Agents for Fluorescence Imaging .

16.1.4 Prospects of Macroscopic Fluorescence Imaging .....

16.1.5 Conclusion

16.2 Bioluminescence Imaging

16.2.1 General Introduction

16.2.2 Production of Light by Living Organisms

16.2.3 Production of Light and Mechanisms

000

000

000

000

000 000 000

Frauke Alves

Department of Haematology and Medical Oncology and Institute for Diagnostic and Interventional Radiology, University Medical Center, Goettingen, Germany

Department Molecular Biology of Neuronal Signals, Max Plank Institute for Experimental Medicine, Goettingen, Germany

J. Bode $(\bowtie)$

German Cancer Research Center (DKFZ), Molecular Mechanisms of Tumor Invasion (V077), Im Neuenheimer Feld 581, Heidelberg 69120, Germany

Department of Molecular Biology of Neuronal Signals, Max-Planck-Institute for Experimental Medicine, Herrmann-Rein-Str. 3, Goettingen 37075 , Germany e-mail: j.bode@dkfz.de

P. Cimalla • E. Koch

Klinisches Sensoring und Monitoring, Klinik für Anaesthesiologie und Intensivtherapie, TU Dresden,

16.2.4 Luciferase as a Reporter Gene for In Vivo Imaging . 000

16.2.5 Functions of Bioluminescence ................... 000

16.2.6 Conclusions ................................................ 000

\subsection{Optical Coherence Tomography} (OCT)

16.3.1 Tissue Properties and Ballistic Photons ..... 000

16.3.2 Coherence, Interferometry, and Diffraction

16.3.3 Fundamental Principles of Optical Coherence Tomography

16.3.4 Extended Functionalities ............................. 000

16.3.5 OCT for Small Animal Imaging ................. 000

16.3.6 Discussion and Conclusion ......................... 000

I. Hilger $(\bowtie) \bullet$ Marc A.M.J. van Zandvoort Universitätsklinikum Aachen, Aachen, Germany

M. Hofmann $(\bowtie) \bullet$ V. Jaedicke

Photonik und Terahertztechnologie, Ruhr Universität Bochum, Bochum, Germany

K. Licha $(\bowtie)$

Mivenion GmbH, Robert-Koch-Platz 4, Berlin 10115, Germany

e-mail: licha@mivenion.com

T. Rademakers $(\bowtie)$

Cardiovascular Research Institute Maastricht (CARIM), Maastricht University, Maastricht, The Netherlands

D. Razansky $(\bowtie)$

Institute for Biological and Medical Imaging, Technical University of Munich and Helmholtz Center Munich, Munich, Germany 
16.4 Intravital Imaging in Small Animals ..... 000

16.4.1 Classical Intravital Imaging ........................ 000

16.4.2 Intravital Multiphoton Imaging ................... 000

16.4.3 Perspectives and Considerations ................ 000

16.5 Optoacoustic Imaging ................................. 000

16.5.1 Introduction ................................................. 000

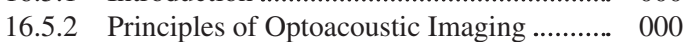

16.5.3 Multispectral Optoacoustic Tomography .... 000

16.5.4 Optoacoustic Imaging Instrumentation ...... 000

16.5.5 Small Animal Imaging Applications ......... 000

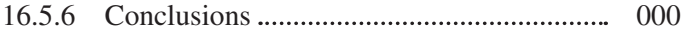

16.6 Optical Probes ............................................. 000

16.6.1 Introduction: Optical Probes for

Animal Imaging ..... 000

16.6.2 State-of-the-Art Technologies ..................... 000

16.6.3 The Choice of Parameters ............................ 000

16.6.4 Conclusion and Outlook ................................. 000

16.6.5 Laboratory Protocols .................................... 000

References ........................................................... 000

\begin{tabular}{|c|c|c|c|}
\hline \multicolumn{2}{|c|}{ Abbreviations } & PSMA & Prostate-specific membrane antigen \\
\hline & & $\begin{array}{l}\text { QDS } \\
\text { SPECT }\end{array}$ & Single-photon emission computed \\
\hline $\begin{array}{ll}\mu \mathrm{M} \\
\mathrm{AP} 23\end{array}$ & Mlicromolar & SPEC I & $\begin{array}{l}\text { Single-photon emission computed } \\
\text { tomography }\end{array}$ \\
\hline$A D$ & Amyiora precursor protent-25 & TRP & Tumor-to-backor \\
\hline $\begin{array}{l}\text { AD } \\
\text { apoE }\end{array}$ & $\begin{array}{l}\text { Alzheimer's disease } \\
\text { Apoenzyme }\end{array}$ & uMUC-1 & Underglycosylated mucin-1 \\
\hline$A B$ & Amyloid beta & UV-vis & Ultraviolet-visible \\
\hline BODIPY & Boron-dipyrromethene & VEGF & Vascular endothelial growth factor \\
\hline $\mathrm{CCD}$ & Charged-coupled device & & receptor \\
\hline CCK2 & Cholecystokinin-2 & WT & Wild type \\
\hline CEA & Carcinoembryonic antigen & Zn-DPA & Zinc-trisodium-pentetate \\
\hline $\mathrm{CT}$ & Computed tomography & $\mu \mathrm{m}$ & Micrometer \\
\hline CysLT1 & Cysteinyl leukotriene-1 & & \\
\hline $\mathrm{Da}$ & Dalton & & \\
\hline DNA & Deoxyribonucleic acid & & \\
\hline
\end{tabular}

EGFR Epidermal growth factor receptor

EPR Enhanced permeability and retention effect

FDA Food and Drug Administration

fmol Femto mol

FMT Fluorescence-mediated tomography

FRET Fluorescence energy transfer

IC50 Inhibitory concentration of how much of a particular drug (inhibitor) is needed to inhibit a given biological process by half

ICG Indocyanine green

MMP Matrix metalloproteinase

MRI Magnetic resonance imaging

NADPH Nicotinamide adenine dinucleotide phosphate

NIR Near-infrared

nM Nanomolar

PBS Phosphate buffered saline

$\mathrm{pH} \quad$ Potential of hydrogen (strength of hydrogen)

\subsection{Fluorescence Imaging}

Ingrid Hilger

This chapter sets out to (a) describe the main techniques employed in current preclinical and clinical applications of fluorescence imaging, (b) define important aspects of using fluorescence reporters, and (c) describe current applications to diseases with high socioeconomic relevance.

\subsubsection{Introduction}

Fluorescence is the phenomenon by which certain molecules under illumination absorb light of a specific wavelength and normally react by emitting light of a comparatively longer wavelength.

This phenomenon relies on the production of excited single states. An electron in the excited 
orbital is paired with a second electron (of opposite spin) in the ground-state orbital (Lakowicz 1999). A photon is emitted as a result, causing a rapid return to the ground state. Compared to phosphorescence (i.e., the emission of light from triplet excited states), fluorescence emission rates are very high, with fluorescence lifetimes in the $10 \mathrm{~ns}$ range.

Fluorescence occurs quite frequently in nature, in minerals such as benitoite, gypsum, and scapolite, for example. They emit visible light upon being illuminated by invisible ultraviolet light, X-rays, or electron beams. A number of marine animals - for example, lancelets, corals, jellyfish, and marine fish (Sparks et al. 2014) - are also capable of fluorescing, as are some land animals, namely, butterflies, parrots, etc.

Humans have exploited fluorescence by constructing chemical sensors, biological detectors, and cosmic ray detectors as well as by designing fluorescent lamps, etc. Fluorescence has also entered the field of life sciences and medicine, particularly for imaging purposes, as in microscopy. Initially, during the early twentieth century, this technique was primarily used to analyze selected tissue and cell samples with corresponding fixation. About 20 years later, a fluorescent contrast agent, fluorescein, was developed for use in neurosurgery applications (Moore et al. 1948) to improve the detection of brain tumors in patients. Expanding beyond microscopy, fluorescence featured prominently in the development of sophisticated mesoscopic and macroscopic optical imaging systems. This classification of optical imaging modalities from microscopy to macroscopy was originally proposed by (Ntziachristos 2010) and was based on the tissue depth at which they operate (see below).

The success of fluorescence optical imaging methodology is in large part attributable to its (1) ease of use, (2) low cost, and (3) high sensitivity (up to the fmol range of detected dye molecule, depending on the method used) without requiring the use of radioactive tracers.

Nevertheless, even with all the technical developments of the last years, researchers and users still face the basic challenge that all tissues scatter light, which also leads to reduced resolution and difficulties in image formation (Yodh and Chance 1995). In particular, when exciting a specific optical probe localized within tissue, the signal intensity emerging from it will be affected by absorption and scattering (Arridge 1999; Schotland and Leigh 1992). To be exact, this means that the fate of excitation and emission photons depends on the tissue's scatter, anisotropy, and refractive indexes (Alfano et al. 1997). Therefore, the detection limits of fluoro-optical technologies within tissues are determined by a photon's main free path and the average angles at which photons scatter during each scattering event as stated by Ntziachristos et al. (2011).

Wavelengths are known to determine the degree of absorption and scattering in tissue and therefore the penetration depth in tissues. Using near-infrared light permits detecting tissue structures deeper under the surface than using visible light. In particular, near-infrared photons (wavelengths between 650 and $900 \mathrm{~nm}$ ) were observed to travel more efficiently through tissues than ones in the visible light spectrum (Jobsis 1977). When wavelengths increase (i.e., red or infrared region of the optical spectrum), absorption and scattering of light in tissue are known to diminish. The longer the wavelength is, the lower is the delivered energy of the photons. Absorption is at its lowest between 500 and $800 \mathrm{~nm}$ (Osterman and Schutz-Geschwender 2007). By contrast, when increasing wavelength above $900 \mathrm{~nm}$, increasing absorption by water results in markedly reduced transmission of light.

In normal tissue, the principal absorbers are water, oxyhemoglobin, deoxyhemoglobin, and lipids. The extent of absorption depends on the molar concentration of each constituent. For example, in a tissue with $8 \%$ blood volume and $29 \%$ lipid content, the dominant absorber will be hemoglobin, accounting for $39-64 \%$ of total absorption at NIR wavelengths (Lim et al. 2003).

In addition to photon absorption and scattering in living tissues, tissue autofluorescence can also noticeably limit contrast in fluorescence optical imaging. The absorption of a photon is obtained by summing up all absorbing constitu- 
ents present in tissue. Under visible light, fluorescence of intrinsic fluorophores such as NADPH, collagen, elastin, and flavin, etc., may occur (Frangioni 2003; Kovar et al. 2007), potentially interfering with that of xenogen reporters in the visible range of the light spectrum. NIR light solves this problem by reducing the autofluorescence background of intrinsic fluorophores.

With respect to NIR regions, tissue depths of several centimeters can be achieved, depending on the type of tissue, e.g., $10 \mathrm{~cm}$ in the human breast (Buehler et al. 2010a).

Most applications in recent years focused on preclinical whole-body imaging using nearinfrared light. In this context, strategies employing NIR light typically included labeling specific high-affinity ligands with NIR fluorochromes, nanoparticles, and the like. Depending on the macroscopic imaging method used, less than 100 fmol of an NIR dye can be detected (Ntziachristos 2011). At present, a growing number of papers describe the introduction to the clinical field of fluorescence imaging, particularly for intraoperative (van Dam et al. 2011), endoscopic, and laparoscopic imaging applications (van der Poel et al. 2011) and intra-arterial imaging (Yoo et al. 2011).

\subsubsection{In Vivo Fluorescence Imaging Systems}

In this section, the focus will be on macroscopic fluorescence imaging that allows probing tissues to depths greater than $1 \mathrm{~cm}$. Macroscopic imaging lets researchers probe organs, tissues, or entire small animals. For these applications, macroscopic fluorescence imaging systems have been used primarily as stand-alone devices for preclinical imaging. Recently, combining them with other imaging methods, such as MRI and CT, has also been suggested as a means for correlating the fluorescence signals with anatomical information. A comprehensive review of the advantages and current limitations of macroscopic imaging is found in (Ntziachristos 2010, 2011). The main imaging strategies are fluorescence reflectance imaging and fluorescence-mediated tomography, both of which will be discussed in greater detail below.

\subsubsection{Epi-illumination or Reflectance Imaging}

Preclinical fluorescence imaging devices include, among others, quite basic epi-illumination (photographic) systems. The typical epi-illumination system consists of a sensitive charge-coupled device (CCD) camera, a filter system operating at defined wavelengths, illumination systems for fluorophore excitation, macro lens systems, a lighttight imaging chamber, and the software needed to control the device. Among CCD cameras, silicon CCD cameras offer the best cost/ performance ratio, but their sensitivity is rather poor above $800 \mathrm{~nm}$. Increased sensitivity up to $900 \mathrm{~nm}$ can be achieved with more expensive arsenide CCD cameras. Also available are gallium arsenide cameras, but their sensitivity below $1,000 \mathrm{~nm}$ is poor (Frangioni 2003).

Typically, spectral bandwidths - resulting from filtering white light or use of a laser source or laser diode - are used to excite the fluorescing probes. The light source can also be used to produce an illumination image of an animal. The light falling on the surface of the tissue or animal will propagate into it for several millimeters (i.e., in the NIR) and excite the fluorophores. Similarly, the produced fluorescence signals will propagate to the surface. Epi-illumination systems can be used for both fluorescence and bioluminescence imaging.

Characteristic of epi-illumination systems is that the components used to illuminate and detect emitted fluorescence are lined up on the same side of the object of interest. The technique is mainly useful in detecting superficial tumors (e.g., tumor xenografts) in laboratory animals for preclinical research, since, depending on the type of tissue, probing is limited to depths of 3-7 mm from the tissue surface. In clinical practice, these systems are appropriate for open surgery and endoscopic procedures. The particular advantages are that the systems are simple to implement and operate. However, the fluorescence images will not exactly reflect probe localization since, as mentioned earlier, their fluorescence emission will be closely affected by absorption and scattering. Absorption and scattering will also impair full quantification of fluorescence signals (Boot et al. 2008). Therefore, semiquanti- 
tative analysis of the fluorescence intensities is often performed to obtain rough approximations, particularly when taking repeated images from the same animal during the course of the disease (Haedicke et al. 2013; Kossatz et al. 2013). With this, the relative changes in probe accumulation can be deduced. Nevertheless, it must be taken into account that areas of high vascularization in tumors will certainly favor unspecific probe accumulation but will also absorb fluorescence emission due to the presence of blood (Huisken et al. 2004). The resolution of epi-illumination technique is approximately $2-3 \mathrm{~mm}$ with sensitivity in the $\mathrm{nM}$ to $\mu \mathrm{M}$ range.

\subsubsection{Fluorescence-Mediated Tomography}

Another type of fluorescence imaging methods utilizes fluorescence-mediated molecular tomography (FMT). In this method, the tissue of interest is illuminated from multiple angles, then the emitted fluorescence and excitation photons that have propagated through the tissue are collected and registered in different spectral bands. Using appropriate mathematical processing tools on the raw data, this method permits creating three-dimensional quantitative images of fluorescent probe localizations in the area of interest (Ntziachristos 2010). A comparatively high resolution can be achieved by illuminating the tissue surface with point light sources and by applying theoretical models and assumptions of photon propagation. In general, the sensitivity is in the nM-range, with an in vivo detection limit of 1 picomol fluorochrome $(10 \mathrm{nM})$ (Ntziachristos et al. 2002). The resolution is at least $500 \mu \mathrm{m}$, and, despite some improvements, photon scattering remains a challenge. Consequently, to correlate the fluoro-optical signals with anatomical localization, the use of hybrid systems has been suggested. Feasible combinations include FMT with CT or MRI. These combinations can also be used to further improve image reconstruction and visualization (Ale et al. 2013; Gremse et al. 2014).

\subsubsection{Multispectral Imaging}

A particular advantage of fluorescence optical imaging is its ability to generate multiple images using different spectral regions or, alternatively, by applying methods for resolving different spectra (multispectral imaging). Different multispectral decomposition methods suggested can be applied either to the excitation light or to the fluorescing light. Corrections for absorption characteristics of various tissues, scattering variations, and depths are feasible. These methods have been summarized in the following review (Ntziachristos 2010). The method has been applied to detect more than one molecular marker in cancer (Kossatz et al. 2013), cancer therapeutic efficiency (Haedicke et al. 2013) or for in vivo imaging of FRET (Busch et al. 2012) in edemas, etc. (Fig. 16.1).

\subsubsection{Other Fluorescence Imaging Methods}

Optoacoustic imaging is not based directly on fluorescence emissions by fluorophores but instead involves the detection of signals via optoacoustic impulses (for more details, see Sect. 16.5). The method allows the identification of intrinsic chromophores in tissue, such as oxyhemoglobin and deoxyhemoglobin (Allen and Beard 2006a; Eghtedari et al. 2007; Kolkman et al. 2004; Zemp et al. 2007). Optoacoustic imaging can also detect fluorochromes and fluorescent nanoparticles familiar from epiillumination techniques (Razansky et al. 2007). Sensitivity is in the $\mathrm{nM}$ to $\mu \mathrm{M}$-range, resolution is above $20 \mathrm{~nm}$, the latter being limited by the attenuation of acoustic frequencies in the tissue (Ntziachristos 2010).

Further details on other fluorescence imaging methods such as bioluminescence imaging, optical coherence tomography, intravital microscopy can be seen in the following chapters of these book Sects. 16.2, 16.3, 16.4, 16.5, and 16.6.

\subsubsection{Contrast Agents for Fluorescence Imaging}

\subsubsection{General Features}

In addition to the fluorescence imaging devices themselves, appropriate contrast agents are an important complementary tool needed for probing organs, tissues, and whole animals. Most of the fluorescing contrast agents are aromatic 


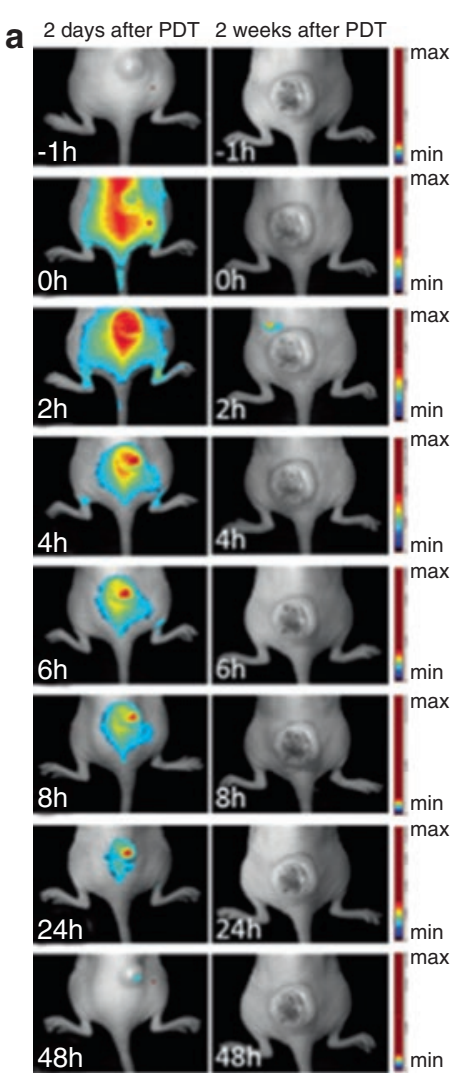

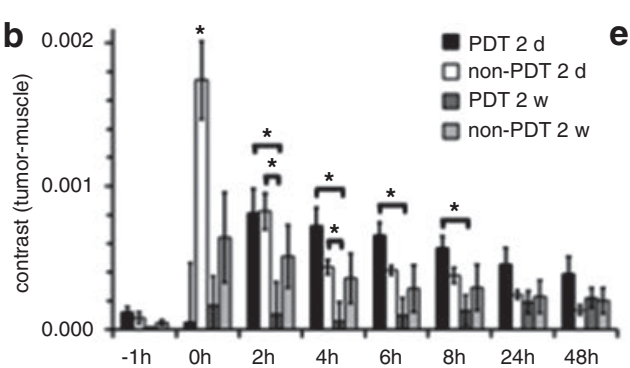

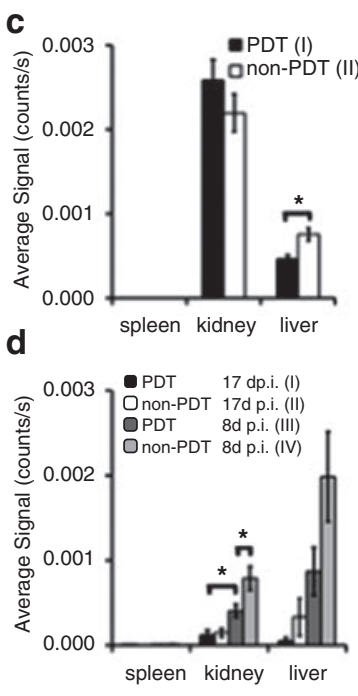

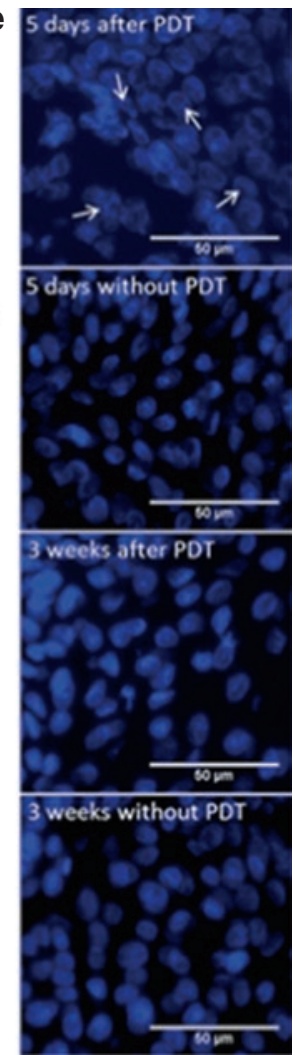

AU5 Fig. 16.1 Multispectral fluorescence imaging of apoptotic cells in tumors via photodynamic therapy. (a) Representative composite images of tumor-bearing mice injected with a fluorescent annexin $\mathrm{V}$ probe at 2 days (left column) or 2 weeks (right column) after therapy. (b) Semiquantitative analysis of fluorescence signals in tumors in treated or non-treated mice up to $48 \mathrm{~h}$ postinjection, $2 \mathrm{~d}$, 2 days, or $2 \mathrm{w}, 2$ weeks $(* p<0.05)$. (c) Biodistribution of the fluorescent annexin V 5 days after therapy and 3 days post probe injection in PDT-treated (black) and non-treated (white) mice, ex vivo organ fluorescence, ${ }^{*} p<0.05$. (d) Biodistribution of the annexin $\mathrm{V}$ probe at 3 weeks after therapy and 17 days (black bars) and 8 days (dark gray bars) after probe injection in treated mice or 17 days (white bars) and 8 days (light gray bars) after probe injection in non-treated mice, fluorescence signals of the organs, $* p<0.05$. (e) Cell nuclei of the cryo-frozen tumor slices 5 days (upper images) and 3 weeks (lower images). Arrow: condensed chromatin (Hoechst stain) (From Haedicke et al. (2013) with permission from Elsevier) organic molecules (Fig. 16.2). Ideal fluorescence reporters should emit fluorescence in the nearinfrared spectral range, as already noted. The fluorescent dyes most in use for in vivo imaging range between 550 and $900 \mathrm{~nm}$ in the diagnostic window. Typical examples are cyanines and their derivatives (i.e., Cy5.5 with an emission wavelength of $695 \mathrm{~nm}$ (Kovar et al. 2007), hemicyanine dyes (Busch et al. 2012), and others. Increasingly popular are also Alexa Fluor 680, IRDye 680, and IRDye 700DX as well as the red chromophores Alexa 750 and IRDye $800 \mathrm{CW}$ (Kovar et al. 2007; Panchuk-Voloshina et al. 1999). Other fluorescent dyes are derivatives of squaraines, phthalocyanines, porphyrin, and borondipyrromethene analogues (BODIPY). In recent years, much attention has been paid to the advent of quantum dots (QDs), nanoparticles constituted of heavy metals such as cadmium and selenite (see below).

A shared characteristic of fluorescent dyes is their high applicability potential, since (1) large-scale chemical synthesis is fundamentally feasible and (2) they are suited for rapid and efficient conjugation with specific molecules, such as DNA primers, nucleotides, proteins, small molecules, amino acids, etc. Most recently, modifications of their basic chemical structure 


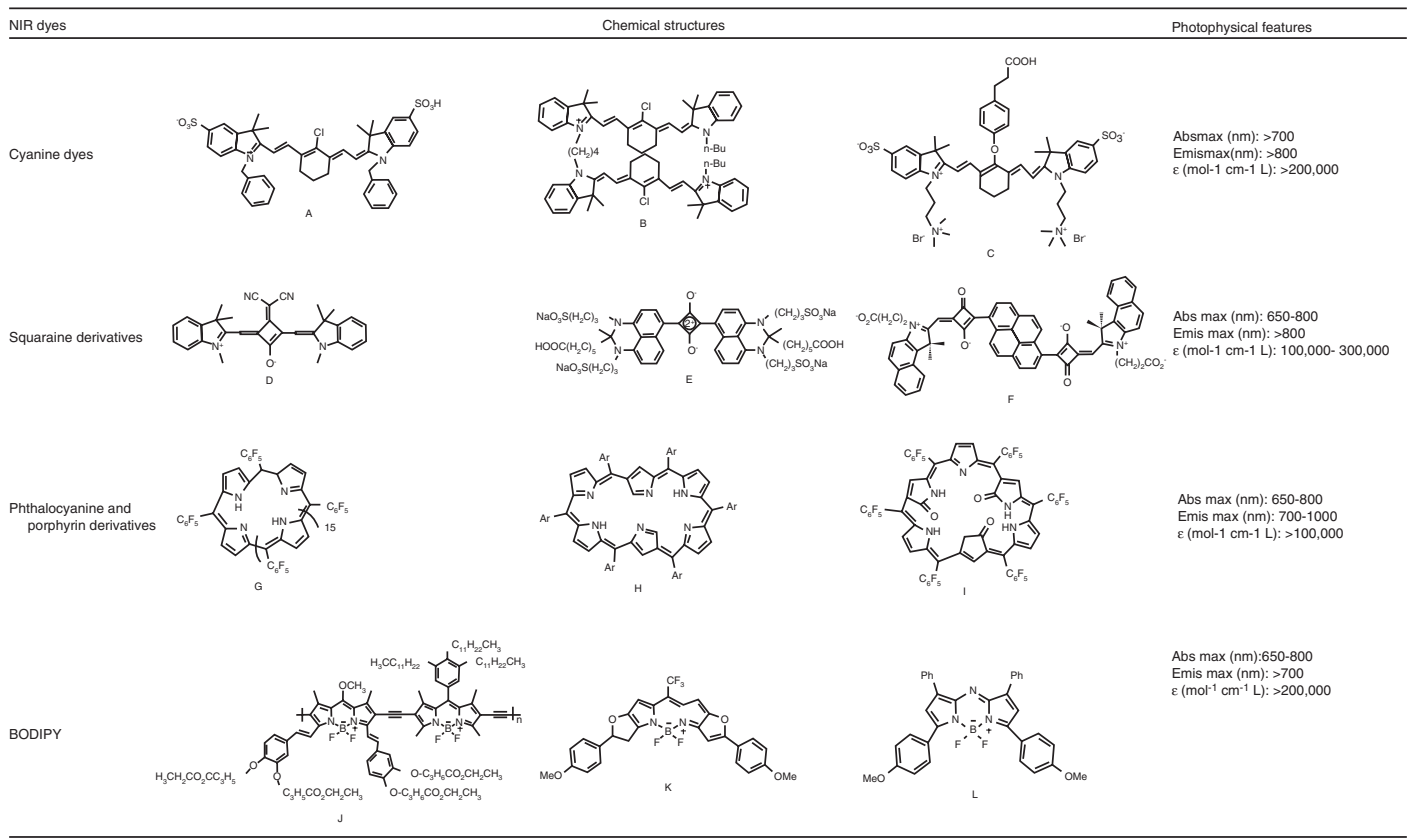

Fig. 16.2 Several of the newly developed NIR fluorescent dyes (From Luo et al. (2011a) with permission from Elsevier)

have produced improved chemical stability and photostability, long fluorescence life, and increased solubility with less aggregation propensity in biological systems (see below). Of particular significance is their adequate separation between excitation and emission wavelengths (Stokes shift), permitting effective filtering of emitted fluorescence from excitation light.

To be detected properly, fluorescence dyes should exhibit high quantum yields. The fluorescence quantum yield is defined as the efficiency of the fluorescence process. Mathematically, it is the ratio of the number of photons emitted to the number of absorbed photons. The ideal quantum yield is 1.0, indicating that every absorbed photon leads to a photon emission. In general, one can assume that all dyes between the values of 0.1 and 1 are fluorescent. Accordingly, this holds true for most of the NIRF dyes applied for in vivo imaging purposes, with values between 0.13 and 0.27 (Ohnishi et al. 2005). The highest quantum yields are achieved with QDs (around 0.50) (Frangioni 2003). All compounds below a value of 0.1 lack a detectable fluorescence emission. The values cited here should be regarded as rough estimates of fluorescence detection intensity, since the determination of quantum yield is highly susceptible to the features of the surrounding medium and lack of standardization.

Solubility of fluorescent probes is an important characteristic, as it assures an adequate dispersion in the body after intravenous application. Because of their basic molecular structure, hemicyanine and cyanine dyes lack solubilization. In order to boost it, attempts have been made to couple polar groups, such as sulfonates and others, with the basic configuration. Lack of solubility can lead to aggregation and agglomeration of the dye molecules, resulting in a reduction of the quantum yield (see below). As a result, the quantum dots produced initially were fairly insoluble, having been constructed with inorganic shells. New formulations use polar organic layers to make them water soluble. Due to their heavy metal constituents leading to low biocompatibility and degradability, QDs are restricted to preclinical applications.

Photobleaching is another phenomenon that needs to be considered in relation to the use of fluorescent probes. It is known to be associated with the reduction of fluorescence due to the molecule's destruction in the presence of light required to induce emission of fluorescence. Photobleaching is closely linked to the compound's stability. Most 
of the known fluorophores are susceptible to it, although the light emitted by most fluorophores is adequate for successful optical imaging applications. QDs are more resistant to photobleaching than ICG and hemicyanine dyes.

Nonspecific binding is another important aspect, particularly in connection with detecting target-affine fluorescent probes. Nonspecific binding can occur as a result of interaction with cellular membranes as well as with extracellular matrix materials, etc. Nonspecific binding generally lowers the signal-to-noise ratio. Depending on their molecular configuration, most of the cyanines and hemicyanines have a tendency to bind proteins. For example, four members of the DY-67x cyanine family, composed of the same main chromophore but substituted with a sequentially increasing number of sulfonate groups (n 5 1-4; DY-675, DY-676, DY-677, DY-678, respectively), were shown to exhibit different bovine serum albumin binding constants: that of the most hydrophobic dye, DY-675, was 18 times higher than that of DY-678, the most hydrophilic fluorophore. In vivo biodistribution analysis revealed a considerable influence of dye hydrophilicity on biodistribution and excretion pathways. In this connection, the more hydrophobic dyes, DY-675 and DY-676, accumulate preferentially in the liver, followed by strong fluorescence signals in the bile and gut owing to accumulation in feces, while comparatively hydrophilic DY-678-COOH accumulates in the bladder (Hamann et al. 2011) (Fig. 16.3). QDs with a high number of charged surface groups exhibit pronounced nonspecific binding behavior (Bentzen et al. 2005). In contrast, hydroxylcoated QDs were shown to be involved in a distinct reduction of nonspecific binding compared to carboxylated QDs (Kairdolf et al. 2008).

In order to achieve good signal-to-background ratios, fluoro-optical contrast agents should basically possess high-target affinity, good accessibility to the target molecule, and rapid clearance, since any uncleared molecule will contribute to the background signal. The commonly used dyes ICG, Cy5.5, and Cy7, IRdye800 $\mathrm{CW}$, etc., are cleared very rapidly from the blood (Frangioni 2003). Polar hemicyanine dyes are

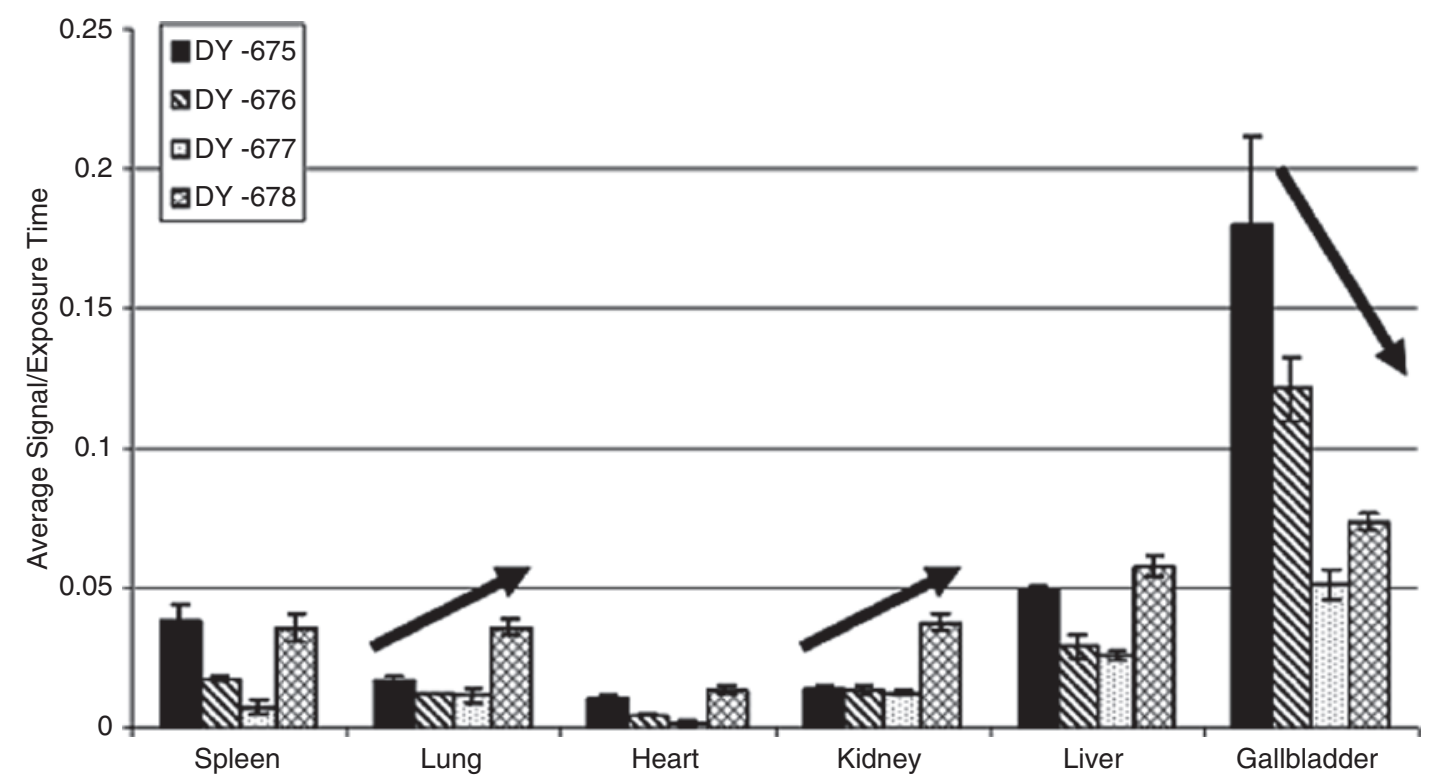

Fig. 16.3 Time-dependent dye organ distribution of one member of the DY-67x cyanine family composed of the same main chromophore, but substituted with a sequentially increasing number of sulfonate groups (DY-675, DY-676, DY-677, or DY-678, respectively) in living male
NMRI mice. Arrows indicate the main accumulation behavior of the dyes with increasing hydrophilicity in selected organs (From Hamann et al. (2011) with permission from Decker Publishing) 
cleared by renal filtration, nonpolar ones, such as ICG and nonpolar hemicyanines via the bile (Hamann et al. 2011; Recknagel et al. 2012). QDs with diameters over $3.5 \mathrm{~nm}$ cannot be cleared by renal filtration, which means that they circulate longer (Frangioni 2003).

Most fluorescent dyes have been shown to have low toxicity. However, so far, ICG is the only fluorescent dye approved by the FDA (Benya et al. 1989). The toxicity of hemicyanine dyes and ICG derivatives, such as Cy5.5, has not been fully evaluated. In some studies, the cytotoxicity and toxicity of Cy5.5 and the hemicyanine dyes were found to be lower than with ICG (Pauli et al. 2009a). QDs might be potentially dangerous because of the presence of cadmium and selenium (Hardman 2006). The features of the fluorescent dyes and QDs have been nicely summarized by te Velde et al. (2010). For more details, see Sect. 16.6.

\subsubsection{The Most Popular Fluorescent Probes: Cyanine Dyes, Their Derivatives, and QDs}

Cyanine dyes are synthetic dyes belonging to the group of polymethines. Other than cyanines with open chains, most of the cyanine molecules for biomedical applications are characterized by the presence of two aromatic heterocycles (symmetric cyanines), each of them containing a nitrogen molecule. Both nitrogen molecules can be part of a heteroaromatic moiety (e.g., pyrrole, pyridine, etc.). The heterocycles are linked by a polymethine bridge. The extension of the molecule by a vinylene moiety leads to a shift of absorption/ emission to a longer wavelength (bathochromic shift) of about $100 \mathrm{~nm}$ (Mishra et al. 2000). In this context, mono- and trimethine cyanines absorb and emit in the visible region of the light spectrum and pentamethines in the near-infrared region.

In general, cyanine dyes and their derivatives are associated with a small Stokes shift and some aggregation in aqueous media (Landsman et al. 1976), which leads to rapid elimination from the blood through the liver. Despite being approved, ICG has in fact recently been reported as inducing transient cytotoxic effects (Ho et al. 2003; Ikagawa et al. 2005; Skrivanova et al. 2006). The introduction of carboxylic and sulfonic acid groups to the basic cyanine structure to address its low solubility in water led to a distinct improvement (Peng et al. 2005; Zhou et al. 2007). Interestingly, the heptamethine cyanine $\mathrm{BHmC}$ that is made up of two cyanine subunits exhibits a distinct increase in fluorescence after protein binding in contrast to solubilization in water (Kim et al. 2005).

Among currently available cyanines, indocyanine green (ICG, Cardiogreen) was already approved in 1958 for use in patients. The small molecule (775 Da) exhibits a short plasma halflife (1-2 min). It is heavily protein bound, has a low quantum yield in plasma, and diffuses readily from the vessels into the interstitium. It has been used for routine tests in retinal angiography (Chang et al. 1995) among others. It is also used in liver function testing (Cherrick et al. 1960; Weissleder and Ntziachristos 2003), for the identification of small and macroscopically invisible liver cancers in real time (Gotoh et al. 2009; Ishizawa et al. 2009), and in rheumatoid arthritis (Schafer et al. 2013). Typically, intravenous dosages of 25-50 mg ICG in saline are used. ICG has also been used to image the cerebrospinal fluid (Sakatani et al. 1997).

Hemicyanines are made up of at least one heterocycle instead of the two that are normally present in cyanine molecules (asymmetric cyanines). The fluorescence quantums of these dyes in aqueous solution depend on the substitution pattern of the benzopyrylium-type end group, which also significantly determines the aggregation tendency. Sterically demanding substituents and negatively charged sulfonate groups reduce the aggregation tendency (Pauli et al. 2011).

Squaraines are made up of an oxocyclobutenolate core and aromatic heterocyclic components at the two ends of the molecule (Volkova et al. 2007). These molecules present a high molar extinction coefficient and good photoconductivity (Dilek and Akkaya 2000; Oswald et al. 2000). However, water solubility is very poor due to their planar and hydrophobic molecular structure. Recently, stable and non-cytotoxic supra- 
molecular adducts of squaraines and the natural carrier protein, i.e., serum albumin, have been suggested for tumor-targeted imaging and photothermal therapy in vivo (Gao et al. 2014).

Phthalocyanines are porphyrin derivatives made up of four pyrrole moieties that were linked together through nitrogen atoms. These molecules are thermally and chemically very stable. Due to the feasibility of replacing the two central hydrogen atoms with metal atoms, plus the added possibility of incorporating substituents at the periphery and axial positions of the molecules, these molecules have been also used in optoelectronics and biomedicine applications (Cammidge and Gopee 2002). Of particular interest are applications as photosensitizers. When illuminated, they produce toxic singlet oxygen capable of killing target (tumor) cells (Haedicke et al. 2013). Several porphyrin derivatives such as porfimer sodium (Photofrin ${ }^{\circledR}$ ), 5-aminolevulinic acid or ALA (Levulan $®$ ), and methyl aminolevulinate (Metvix $\AA$ ), chlorin (Foscan $\left.{ }^{\circledR}\right)$, temoporfin, etc., are FDA-approved photosensitizers for photodynamic therapy. Photofrin is used intravenously for internal cancers, while Levulan $₫$ and Metvix $®$ are applied in skin therapy (MedicineNet).

BODIPY (borondipyrromethene) dyes exhibit high quantum yield and excellent thermal and photochemical stability. However, most BODIPY dyes emit from yellow to deep-red emission with low extinction coefficients. Recent years have seen several attempts to shift the absorption spectrum to the NIR region of the spectrum (Rickert et al. 2010).

Quantum dots (QDs) Compared to organic dyes, QDs possess a series of interesting features such as high-absorption cross section, broadband absorption, narrow and symmetric luminescence band, simultaneous excitation with different emission wavelengths using a single excitation wavelength, etc. (Bruchez et al. 1998; Chan et al. 2002; Wu et al. 2003). Interestingly, by modulating the size of QDs made of a given material, different colors can be achieved. Doing so might make multiplexed imaging with differently sized QDs with the same excitation wavelength a versatile tool in preclinical imaging approaches. These nanoparticles are made up of heavy metal ingredients such as cadmium and selenite. To increase suspension in aqueous solution, nanoparticles are increasingly being coated with an organic material. They exhibit a high extinction coefficient combined with a quantum yield comparable to fluorescent dyes. They are highly stable (low photobleaching effects). In particular, QDs are 20 times brighter and 100 times more stable than traditional fluorescent reporters (Walling et al. 2009). However, their cytotoxicity in biological systems remains to be fully clarified. Additionally, they are quite expensive to prepare; moreover, difficulties in reproducibility and quantification have also been reported (e.g., (Nikoobakht and El-Sayed 2003; Tsung et al. 2006)).

\subsubsection{Prospects of Macroscopic Fluorescence Imaging}

Since it is a macroscopic imaging modality, fluorescence imaging is expected to be limited to the noninvasive detection in vivo of fluorescence signals in preclinical application to whole animals. In clinical settings, it has potential for use in imaging not only parts of the body with good accessibility to light, such as arthritic joints in hands, but also abnormalities in the gastrointestinal tract, gynecological cancers, cardiovascular diseases using specialized catheters, imaging of the eye, and intrasurgical imaging. In addition, it may serve as a valuable tool in the following applications: drug testing, understanding disease mechanisms in basic research, supporting translation of imaging approaches to clinical practice, pre-screening at key points in time during the course of diseases, etc.

\subsubsection{Oncological Imaging}

Most of the original preclinical oncological fluorescence imaging approaches aimed at demonstrating the feasibility of detecting vascular permeability and diffusion via nontargeted probes by exploiting the enhanced permeability and retention effect (EPR) (e.g., Duncan et al. 2006; Maeda et al. 2009) of tumor microvasculature and also at demonstrating the reliability of detecting molecular markers using targeted opti- 
cal probes in whole animals. Active targeting has been achieved by conjugating fluorescent dyes (mostly cyanines or hemicyanines) with specific ligands made up of peptides, proteins, synthetic molecules, antibodies, aptamers, and others. Higher signal-to-background ratios were achieved with this approach when compared to nontargeted probes. Disadvantages of using target-affine probes in oncological imaging include bioconjugation that can affect the fluorescence of several dye molecules, impairment of ligand specificity because of the presence of comparatively large dye molecules when compared to radioactive tracers, and pharmacokinetics of conjugated ligands that may differ from those with free molecules. When using nanoparticles instead of small molecules, accessibility to the tumor interstitium may be impaired due to their comparatively large size (several $\mathrm{nm}$ ). Particularly when imaging structures of the tumor interstitium, using small molecules is advantageous because of their high tissue penetrating behavior and rapid clearance of non-bound molecules from the tumor. Typical examples for active targeting in oncological fluorescence imaging address the tumor cell compartment per se, the tumor environment, extracellular compartment, and neovascularization, such as the detection of PSMA (Liu et al. 2010), folate (Tung et al. 2002), EGFR (Becker et al. 2000), CEA (Hilger et al. 2004), Her-1 and Her-2 (Ogawa et al. 2009), CCK2 receptor and Gut-1-receptor (Kossatz et al. 2013), apoptotic cells (Haedicke et al. 2013), uMUC-1 antigen (Pham et al. 2005), $\alpha v \beta 3$-integrin (Wang et al. 2007a), etc. (Fig. 16.4).

Additional strategies for oncological imaging include the utilization of activatable probes that fluoresce upon activation or the incorporation of fluorescent dyes into (polymeric) nanoparticles with or without targeting moiety. Here, the encapsulation of dye molecules in nanoparticles offers the advantage of having a protective matrix cover the dyes and the ability to amplify the fluorescence signal. Moreover, encapsulation could help improve the stability of the dyes. Nevertheless, nanoparticles have a limited potential for extravasation into the tumor interstitium and might be of particular interest when address-
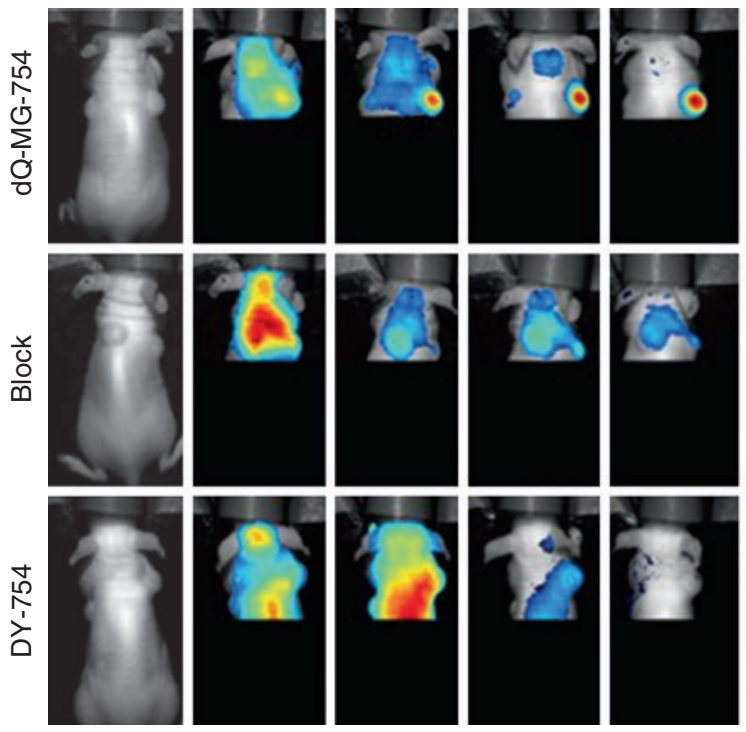

white light

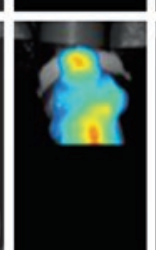

0 h p.i.

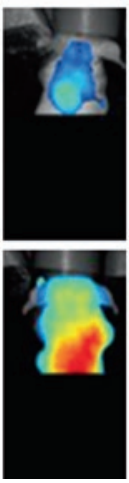

2 h p.i.

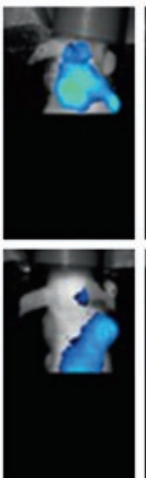

4 h p.i.

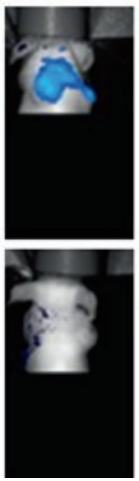

6 h p.i.
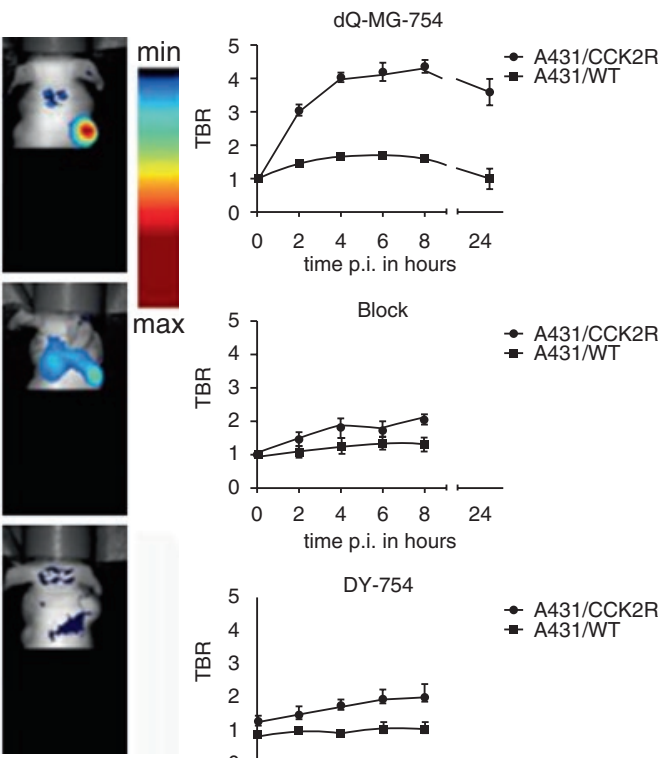

8 h p.i.
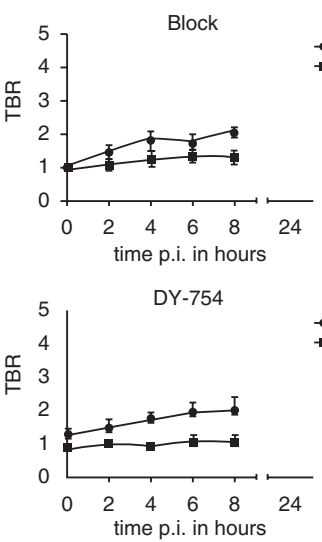

Fig. 16.4 Fluorescence imaging of the cholecystokinin-2 receptor in tumors. NIR fluorescence reflectance images of CCK2R expression in an A431/CCK2R (right flank) and A431/WT (left flank) xenograft model. The left panel displays representative intensity-scaled fluorescence images for each experimental group at indicated time points after probe injection. TBR tumor-to-background ratios (From Kossatz et al. (2013) with permission from Elsevier) 
ing intravascular targets in the tumor. Numerous recent investigations focus on providing nanoparticles with multimodal capabilities for imaging and therapy, given that nanoparticles provide a good platform for multifunctional approaches. Nevertheless, uptake by the mononuclear phagocyte system limits specificity in several organs (liver, spleen, etc.), and reproducibility and the large-scale preparation of such formulations are challenging. For these reasons, application has been limited to the preclinical stage. Typically, silica nanoparticles conjugated with cyanines (He et al. 2007; Haedicke et al. 2015), liposomal nanoparticles for tumor imaging via the EPR effect in combination with photodynamic therapy (e.g., (Bendsoe et al. 2007), chitosan polymers (Backer et al. 2005), etc., have been proposed.

The tumor environment specifically has been addressed with so-called fluorescent activatable probes. The approach exploits the fact that tumor cells exhibit a different $\mathrm{pH}$ and different enzyme activity than normal cells. The corresponding probe does not fluoresce unless activated at the target site, which emits fluorescence from dequenching of the probe's dye molecules. Probes that are $\mathrm{pH}$ sensitive are based on the high sensitivity exhibited by chromophores to protonation and deprotonation (Wang et al. 2010a). Enzymeactivatable probes contain at least two chromophores in proximity to each other and connected by a specific peptide linker that serves as enzyme substrate. Due to the quenching (identical chromophores) or resonance energy transfer (differing chromophores) effects, no or very little fluorescence emission takes place in the non-activated stage. Sample applications include the detection of proteases in tumors such as matrix metalloproteinases, caspases, cathepsins, etc. (Bremer et al. 2001a; Messerli et al. 2004; Tung 2004).

\subsubsection{Imaging of Infections}

Infections represent a profound medical challenge worldwide, particularly when local infections turn into systemic ones. Here (fluorescence) optical imaging could help obtain new insights into the mechanisms of disease and prevention. For example, by utilizing bioluminescence sourced from the luc operon (e.g., from the firefly Photinus pyralis or the sea pansy Renilla reniformis) or lux operons
(Photorhabdus luminescens, Xenorhabdus luminescens) on the bacterial strains of interest, their fate in the body and the disease mechanisms can be tracked via imaging. These operons express the luciferase enzyme which is not naturally present in mammalian cells. To produce light, the enzyme substrate luciferin is applied to the animals. In this context, several researchers found that bioluminescent bacteria could be tracked in vivo in murine models and that the bioluminescence intensity was closely related to the number of viable bacteria (Contag and Bachmann 2002). More recently, in vivo infection with Pseudomonas aeruginosa in an acute murine airway infection model was efficiently tracked with bioluminescence imaging (Munder et al. 2014). To study the liver stage development of malaria in humans and rodents, modified luciferase containing malaria parasites was used to analyze both hepatocytes in culture and in the livers of living mice (Ploemen et al. 2009).

In contrast to the analysis via utilization of genetic reporters, a more straightforward strategy designed for clinical applications is imaging with exogenous synthetic probes that target bacteria selectively. This methodology has a comparatively high potential for clinical translation at least for organs with good accessibility to light. In this connection, a deep-red fluorescent squaraine rotaxane scaffold with two appended (bis(zinc(II)dicolylamine) (bis(Zn-DPA)) targeting ligands were used to track Gram-positive Staphylococcus aureus and Gram-negative Salmonella enterica serovar typhimurium in mice. The bis(Zn-DPA) ligands of the probe had high affinity for the anionic phospholipids and related biomolecules that reside within the bacterial envelope, and they are known to selectively target bacterial cells over the nearly uncharged membrane surfaces of healthy mammalian cells. Imaging of localized infections was possible, and the signal was independent of mouse humoral immune status. The probes did not respond to nonbacterial infections (White et al. 2010). Similarly, optical nanoprobes were designed that were composed of concanavalin $\mathrm{A}$ (Con A) as a bacterial targeting ligand, a nanoparticle carrier, and a near-infrared fluorescent dye. Using a murine wound and catheter infection model, nanoprobes could rapidly detect and quantify the extent of bacterial colonization 
on wounds and catheters in real time (Tang et al. 2014). Additionally, detection of Staphylococcus aureus infection $\left(5 \times 10^{7}\right.$ cells $)$ in a mouse is possible using a cationic antimicrobial peptide conjugated to a near-infrared dye ICG that targets the anionic surfaces of bacteria. This probe selectively accumulates in bacteria (Liu and Gu 2013).

Similarly, it was found that uptake and hepatobiliary excretion ICG and the hemicyanine dye DY635 are altered during sepsis, namely, by considering different variables including hepatic perfusion, hepatocellular energy state, and functional integrity of transporter proteins. In particular, with respect to hepatocellular transport of both dyes, excretion into bile was significantly delayed for both dyes and resulted in net accumulation of potentially cytotoxic xenobiotics in the liver parenchyma. Transcutaneous assessment of ICG fluorescence by whole-body NIRF imaging revealed a significant increase starting from the 30th minute of ICG fluorescence in the bowel region of the abdomen in sham but not in septic animals, confirming a sepsis-associated failure of canalicular excretion. These results have potential implications for monitoring liver function, critical care pharmacology, and the understanding of druginduced liver injury in the critically ill (Recknagel et al. 2012; Gonnert et al. 2013) (Fig. 16.5).
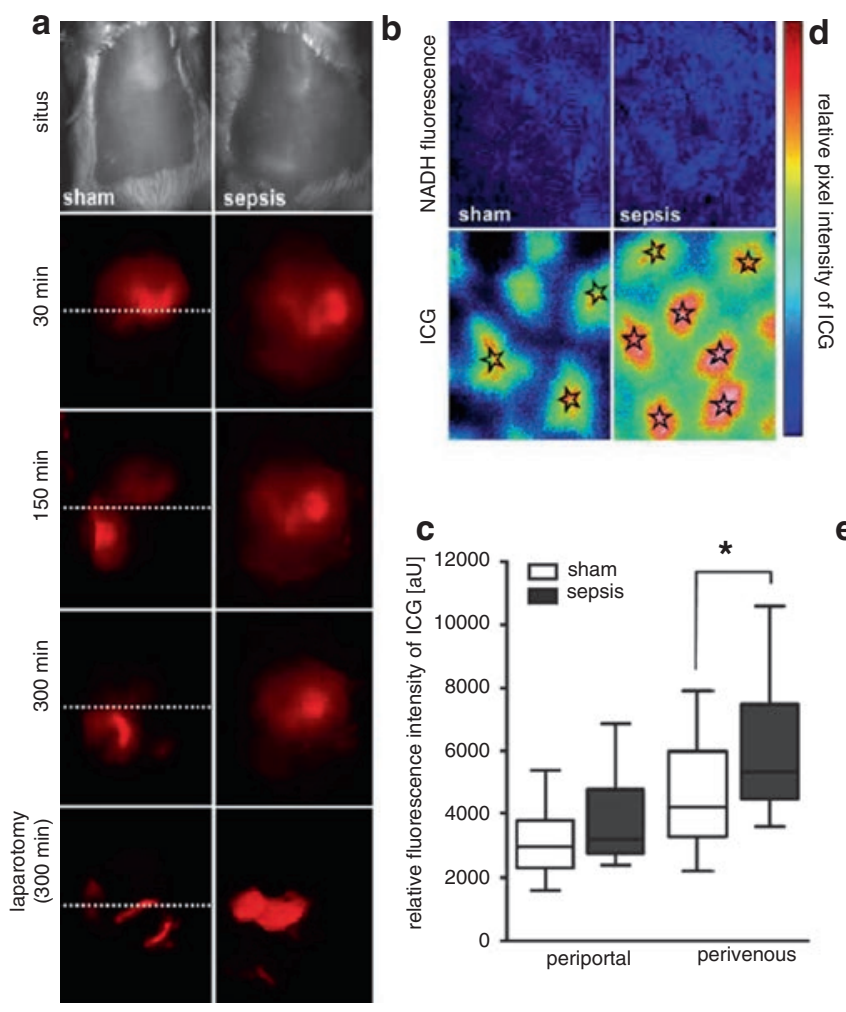

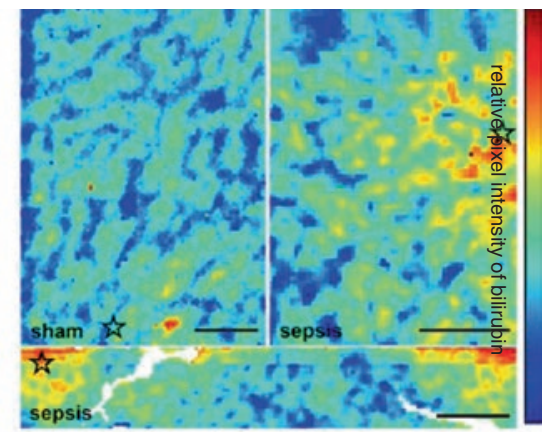

e

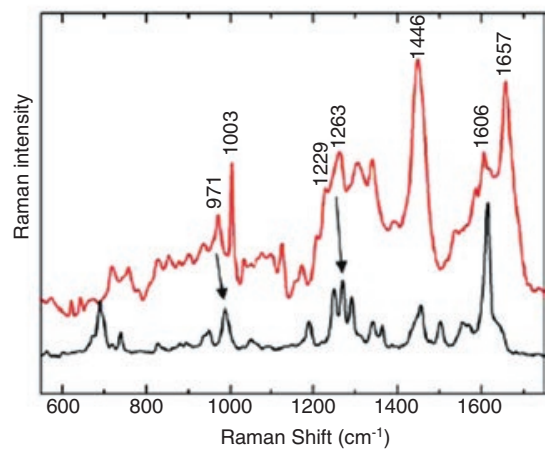

Fig. 16.5 Imaging of sepsis-induced excretory dysfunction, as visualized by accumulation of the xenobiotic indocyanine green and bilirubin. (a) NIRF imaging of ICG at $15 \mathrm{~h}$ after sepsis induction: ICG is eliminated via hepatobiliary excretion into the duodenum in sham animals, whereas the dye accumulates in the livers of septic animals, with an almost absence of fluorescence signal in the gut (the dotted line divides upper and lower abdominal quadrants for orientation). (b) Subsequent epifluorescence microscopic examination of liver surfaces after ICG administration in sham and septic animals. Stars: central veins (pseudo-colored). (c) ICG fluorescence intensities around central veins were significantly higher in septic compared to sham-operated animals $(* p=0.031$ compared to sham). (d) Micro-Raman images of tissue sections from liver sections obtained from sham-operated and septic rats. In the livers of the septic rats, elevated relative intensities of the bilirubin component were found in the perivenous region (stars), but only minor local spots of bilirubin were observed of control animals. Scale bars: $50 \mathrm{~mm}$. (e) Raman spectrum of the bilirubin component (red trace) with crystalline bilirubin for comparison (black trace) (From Recknagel et al. (2012) (open-access article)) 


\subsubsection{Preclinical Attempts at Alzheimer Imaging}

Diagnosis of many neurodegenerative diseases continues to pose challenges. For example, a reliable discrimination of Morbus Alzheimer from other neurodegenerative diseases can only be accomplished post-mortem. For this purpose, the presence of amyloid plaques and tau fibrils in brains of $\mathrm{AD}$ patients is assessed. In living patients, the utilization of Florbetaben $\AA$, an 18 F-labeled amyloid ligand for image-based detection via PET, is currently in a phase III clinical trial (Barthel et al. 2011). The use of optical methods would bypass the use of radioactive tracers in AD image-based detection, which present a challenge given that they require a local cyclotron for production of positron-emitting radionuclides and a synthesis unit to produce radiolabeled agents.

Interestingly, tau and amyloid have been detected not only in the brain (e.g., cortex) but also in the retina (Koronyo-Hamaoui et al. 2011). In contrast to the brain, the eye is easily accessible to light thanks to its natural functions. Therefore, fluorescing small molecules with high affinity for aggregated proteins might be used to report protein aggregation in the retina. In this context, after intravenous injection of a fluorescent probe targeting amyloid plaques and/or tau fibrils, the subsequent illumination of the eye with light of defined wavelengths would presumably reveal the presence of molecular alteration in AD. Nevertheless, there are still several challenges to be overcome with regard to (1) passage of the fluorescence reporter to the brain barrier, (2) clearance of non-bound reporter molecules from the tissue, and (3) demonstrating a verified correlation of pathological changes in the brain and (cognitive) symptoms with pathological abnormalities in the retina. Of interest in this connection are several novel probes based on the bis(arylvinyl)pyrazine, bis(arylvinyl)pyrimidine, and the asymmetric hemicyanine family molecular structure. Among these, bis(arylvinyl)pyrazines represented the most promising UV-vis probes in terms of biocompatibility. Regarding their affinity to $A \beta$ and tau, defined bis(arylvinyl) pyrimidines are the best ligands, endowed with good prospects for AD detection. Additionally, asymmetric hemicyanine exhibited good selectivity for amyloid plaques and tau fibrils at a level similar to that of bis(arylvinyl)pyrazines as well as low cytotoxic effects on human cell lines within the investigated NIR dyes. The data provides a solid base for further investigations of their applicability to image-based detection of AD in vivo (Bolander et al. 2012). Moreover, $a$ series of rhodanine-3-acetic acids was synthesized and shown to bind to neurofibrillary tangles with a comparably high binding affinity (e.g., IC50 = $19 \mathrm{nM}$ ) (Anumala et al. 2013). Lastly, trimethine cyanine dyes bind to tau fibrils ( $\mathrm{Gu}$ et al. 2013).

In other studies, oxazine dyes (Hintersteiner et al. 2005) have been used to track Aß in AAP23 transgenic mice, as have thiobarbitals (Okamura et al. 2011). The dimethylamine structural signature seems to favor binding affinity of compounds to $A \beta$, and it is likely that this functionality is important for $\mathrm{A} \beta$ interaction. Dimethylamines can be found in chalcones, benzothiazoles, or imidazopyridines among others (Eckroat et al. 2013). In addition, other small-molecule fluorescent probes as reporters of amyloid formation, including single-molecule determinations such as JC-1, DCVJ, ANS derivatives, and luminescent conjugated polymers as well as site-specific probes such as pyrenes, have been suggested (Bertoncini and Celej 2011).

\subsubsection{Imaging of Inflammation}

Inflammation is basically associated with the release of exogenous and endogenous chemical mediators after tissue injury. Exogenous mediators (e.g., microbial peptides) act as chemoattractants which in turn recruit neutrophils to the target site. Typically, their aim is to phagocytize invading microorganisms and cellular debris. These response functions protect the host and normally they are self-limiting. Once the xenogen material has been removed, the inflammatory reaction will be resolved. In this context, leukocytes and debris from inflamed sites are removed in order to return to homeostasis. This tightly regulated biochemical and metabolic process can become chronic if the resolution of 
the inflammation process is altered (Serhan et al. 2008).

Inflammation in the body can also be triggered by diverse intrinsic pathophysiological alterations leading to tissue lesions. A typical example is atherogenesis in blood vessels. In such a case, the inflammation process is triggered by infiltration of atherogenic lipoproteins into endothelial cells. This process ultimately leads to the formation of plaques. Acute thrombotic closures of coronary arteries can occur as a result of ruptures of the fibrous cap and exposure of the thrombogenic core to the blood (Jackson 2011).

For this reason, special attention was devoted to the examination of inflammatory plaques via imaging, since early detection and therapy can help reduce cardiovascular mortality and morbidity. To this end, fluorescence imaging was related to the development of fluorescence cardiovascular catheters for clinical application and/or to unveil important mechanisms of plaque formation in preclinical research activities designed to provide new insights into prevention and therapy. Specific fiber systems have been proposed; initially, they were based on one-dimensional systems and more recently on rotational ones. These developments have been summarized in several technical reviews (Jaffer et al. 2007; Lipinski et al. 2006; Nahrendorf et al. 2007). From the pathobiological point of view, the activity of matrix metalloproteinases and cathepsins, which are known to play an important role in rupture of vulnerable plaques, has been assessed (Chen et al. 2002). Subsequently, MMP-2, MMP-9, and cathepsin $\mathrm{K}$ und $\mathrm{S}$ were also efficiently imaged in atherosclerotic apoE mice (Jaffer et al. 2007; Deguchi et al. 2006; Galande et al. 2006) in preclinical research approaches using FMT, which provided deeper tissue penetration capabilities compared to epi-illumination techniques. Additionally, intensive research on the mechanisms of atherosclerosis and thrombosis formation is being performed at the high-resolution and high-speed intravital microscopic level, as reviewed by (Taqueti and Jaffer 2013).

Rheumatoid arthritis, a serious disease which can lead to pronounced activity limitation, disability, and reduced quality of life, is also known to be associated with inflammation. Diagnosis and individualized therapies are important factors in the fight against this disease. Primarily it is the wrists, hands, elbows, shoulder, knees, and ankles that are affected. Even with different imaging technologies in radiologic routines in use for detecting inflammatory arthritis and osteoarthritis, fluorescence imaging holds great promise since affected joints are located near the body surface and thus are mostly well accessible to light. It is expected that (fluorescence) optical imaging can contribute to detection and quantification of pathomorphologies and pathophysiologies in inflammatory rheumatic diseases such as angiogenesis, hypervascularization, hypoxia, and hypermetabolism - not only in the preclinical but also in the clinical stage. To this end, different optical modalities, such as spectroscopic methods, transillumination imaging, diffuse optical imaging, fluorescence and bioluminescence imaging, photoacoustic imaging, etc., have been analyzed. These research activities have been reviewed by Chamberland 2010. With regard to fluorescence optical imaging, the following have been studied: folate receptors (Chen et al. 2005), the F4/80 antigen present on the surface of macrophages (Hansch et al. 2004), cathepsin B (Lai et al. 2004), fluorescent-labeled leukocytes (Simon et al. 2006), perfusion in arthritic joints depending on the molecular weight of the probes (Dietzel et al. 2013), targeting glycosaminoglycans in cartilage degeneration via dipicolylamine (DPA) probes (Hu et al. 2014), and E-selectin (Jamar et al. 2002; Marshall and Haskard 2002). Image-based detection of rheumatoid arthritis in patients by using ICG as contrast agent is being analyzed in several clinical studies (Schafer et al. 2013).

In other studies, the mechanisms of inflammation have been investigated in different animal models. These encompassed granuloma formation using fluorescence-labeled macrophages (Eisenblatter et al. 2009), presence of CysLT1 in edema models (Busch et al. 2011), selective visualization of liposomal uptake by macrophages upon dequenching in endolysosomes) (Tansi et al. 2013), the occurrence of FRET upon uptake of two exogenous probes in endolysosomes of phagocytes (Busch et al. 2012), and others (Fig. 16.6). 


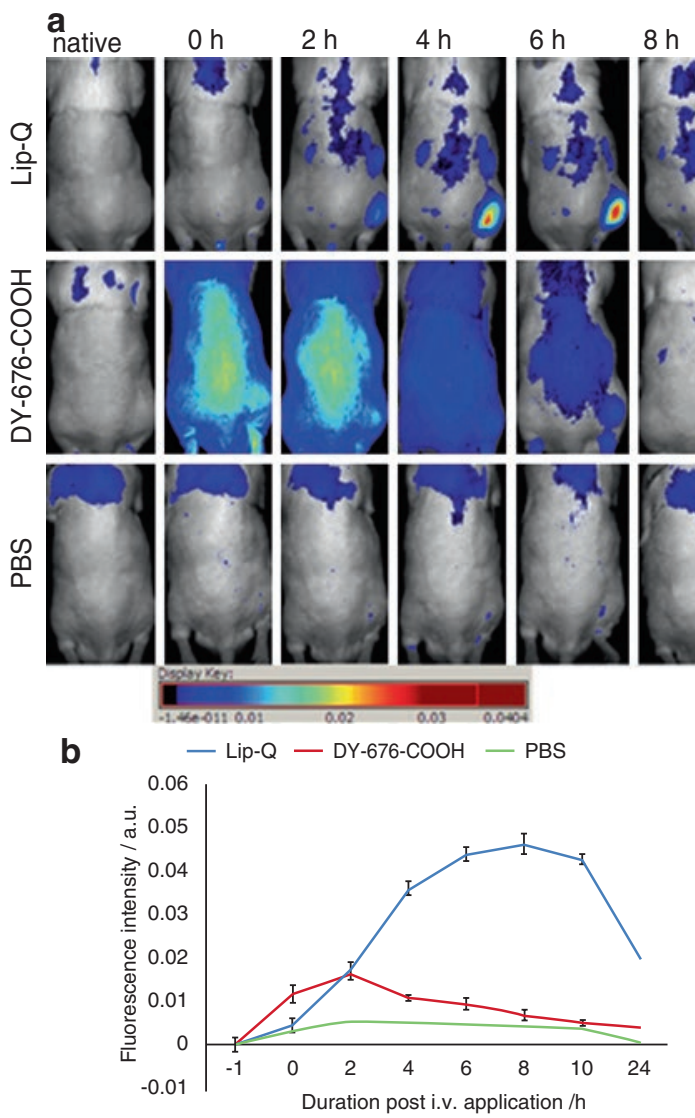

Fig. 16.6 Selective detection of liposomal uptake by macrophages upon dequenching endolysosomes. (a) Representative in vivo NIR fluorescence imaging of edema using quenched liposomes (Lip-Q) or the hemicyanine dye DY-676-COOH (concentration equivalent to Lip-Q content) or PBS, $\mathrm{pH}$ 7.2. (b) Semiquantitative

\subsubsection{Conclusion}

Fluorescence imaging is an active and promising area of research and application in the preclinical and clinical field. NIRF imaging is especially promising thanks to greater tissue penetration depths of light and low interference by tissue autofluorescence. A wide spectrum of NIR fluorophores is now available. When utilizing contrast agents in vivo, several parameters influencing signal-to-background ratio are important for effective imaging applications. These include selection of excitation and emission wavelengths, solubility, photobleaching, target affinity, and pharmacokinetics, to name a few. Although sev-
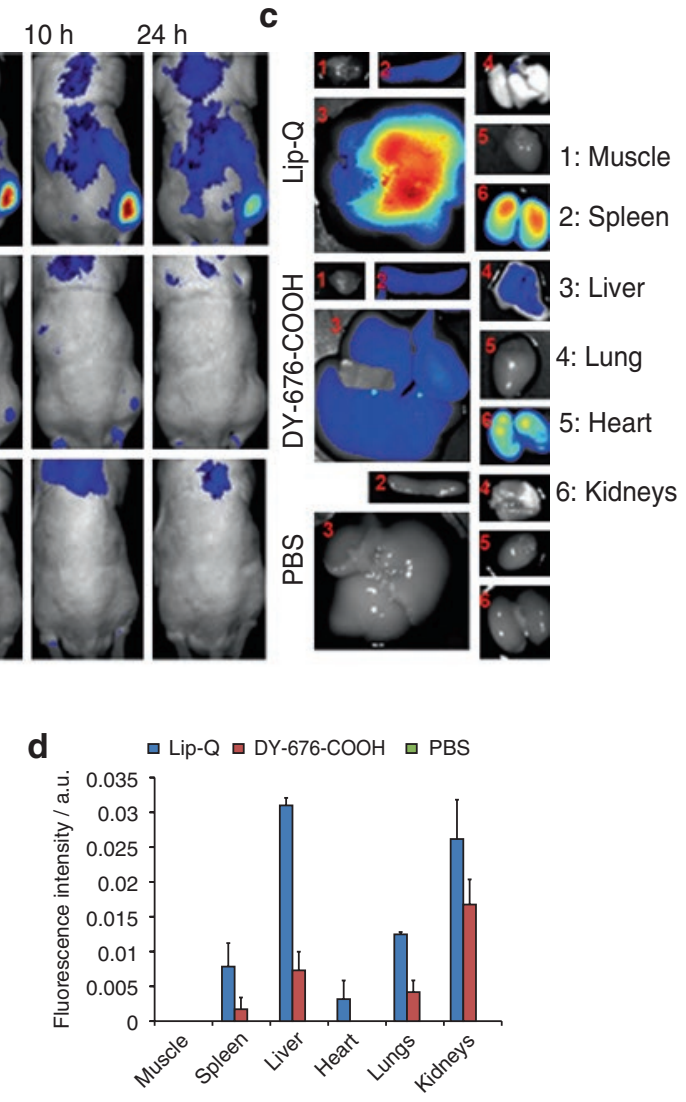

analysis of fluorescence intensities of edema. (c) Biooptical ex vivo images of organs from mice $6 \mathrm{~h}$ post probe application. (d) Semiquantitative analysis of fluorescence intensities of organs after ex vivo NIR imaging (From Tansi et al. (2013) with permission from Wiley and Sons)

eral clinical studies with ICG are under way with a focus on detection of rheumatoid arthritis and certain tumors, the approval of other fluorescent probes, particularly for target-affine reporters, is a large undertaking. In this regard, probes with applicability in several disease frameworks (e.g., vascular targets in inflammation and cancer) will be of higher interest to pharmaceutical companies. With respect to hardware, epi-illumination techniques are widely used in preclinical research, although substantial advances have been made in producing tomographic systems, as well as other sophisticated systems (e.g., optoacoustic imaging). In the long term, clinical research, applications, and discrete fiber and 
intraoperative systems will markedly improve the accuracy of interventional procedures.

\subsection{Bioluminescence Imaging}

Frauke Alves and Julia Bode

\subsubsection{General Introduction}

Optical imaging offers many unique opportunities in order to study biological processes in intact organisms. One option for the measurement of in vivo optical signals is bioluminescence. There has been a rapid growth of bioluminescence imaging applications in small animal models propelled by the approaches in technology during the last years.

In general, bioluminescence refers to the process of visible light emission in living organisms. Light as a form of energy consists of photons with different wavelengths. Light is not only the visible light that can be seen by the human eye with wavelength ranges from around $380 \mathrm{~nm}$ (violet) to about $740 \mathrm{~nm}$ (red), it also means that visible light is only a small part of the total electromagnetic light. This extends from gamma rays $\left(10^{-12} \mathrm{~m}\right)$ through $\mathrm{X}$-rays, ultraviolet, visible, and infrared or near-infrared light up to microwaves and radio waves $\left(10^{3} \mathrm{~m}\right)$ (Sadikot et al. 2005).

There are two major groups where the emission of light can be classified in; one is thermal or heat radiation, whereas the other class consists of nonthermal radiation (Moreno et al. 2014).

\subsubsection{Production of Light by Living Organisms}

A variety of different bioluminescent systems have been identified in nature, every single one requires a specific enzyme and substrate. Living organisms produce light via luminescence. These luminescent species can be found across almost all major taxonomic groups ranging from bacteria via hydrozoa, fungi, or insects up to higher organisms such as fishes. Only few groups exist with no known luminescent forms such as spiders, birds, mammals, or flowering plants (Contag et al. 1998; Haddock et al. 2005, 2010).

The majority of luminescent organisms is of marine origin and indwells the ocean. They can be found all over the ocean depth, but the majority lives in the disphotic zone from 200 to $1000 \mathrm{~m}$ where only a small part of the sunlight from the surface reaches this area. The seawater above absorbs most wavelengths of light in the range of red, orange, and yellow. Only the light with range of blue or blue green light with a short wavelength of about $475 \mathrm{~nm}$ and more energy reaches the deeper area of the ocean, an almost completely dark place. That is why many of the organisms adapted to life and now have the ability to produce their own light (Warrant and Locket 2004). Forms of luminescent terrestrial life can be found all over the world where they indwell different habitats and where they use light for diverse reasons. For example, swarms of glowworms or fireflies dance in the dark in order to impress the sexual partner with the blinking light (Li et al. 2013a; Close et al. 2011).

Armillaria species are other examples for terrestrial luminescence that is the most common source of foxfire. Out of about 40 known Armillaria species, five have been described as luminescent. The mycelium of one single Armillaria is able to reach tremendous dimensions with cases describing covering an area as large as $9 \mathrm{~km}^{2}$ (Zhao et al. 2008; Mendes et al. 2008; Desjardin et al. 2008; Miller et al. 2005).

\subsubsection{Production of Light and Mechanisms}

In 1885, a French scientist, Raphael Dubois, verified the idea of bioluminescence being a result of a chemical process. He made a paste of the luminescent material from the clam Pholas and suspended it in cold water leading to the production of a glowing solution. Additionally, Dubois was able to show that this reaction was repeatable with extracts of the click beetle (Pyrophorus). Out of his studies, Dubois concluded that bioluminescence was chemical in nature and that there 
is an organic molecule involved. He named this molecule luciferin and the responsible enzyme luciferase (Poisson 2010; de Wet et al. 1985).

Decades later the biochemical reaction of bioluminescence, when energy is converted into light, was solved. The substrate luciferin is oxidized by molecular oxygen, whereas the enzyme luciferase catalyzes the reaction toward a nonreactive product and the release of photons. Figure 16.7 shows a simplified scheme of the biochemical reaction.

In detail, luciferin is oxidized to oxyluciferin by luciferase - oxygen and ATP are needed for the reaction as well.

The most commonly used bioluminescent reporter for research purposes is luciferase from the North American firefly (Photinus pyralis). Other useful luciferases have also been cloned from jellyfish (Aequorea), sea pansy (Renilla), corals (Tenilla), click beetle (Pyrophorus plagiophthalamus), and several bacterial species (Vibrio fischeri, V. harveyi) (Hastings 1996).

In comparison to firefly and click beetle luciferase, Gaussia luciferase and Renilla luciferase use coelenterazine and oxygen. Here ATP is not needed since coelenterazine alone is a highenergy molecule that offers the energy for the reaction (Soling et al. 2004). All reactions have in common that they need oxygen.

During the reaction, a peroxyluciferin is built as an intermediate that provides energy for excitation. The peroxyluciferin holds the energy coming from the reaction only for nanoseconds, and afterward the energy is released in the form of a photon.
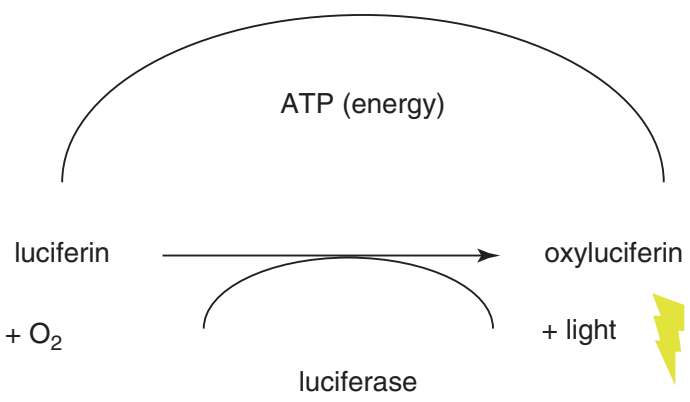

Fig. 16.7 Oxidation of luciferin and catalyzation of the reaction through luciferase. The side product light can be detected
With awareness of this phenomenon, different luciferases were generated by inducing mutations at important amino acids close to the enzymatic gap in order to emit light with different wavelengths. The energy conversion efficiency is with more than $80 \%$ of energy transformed into light that produces close to one photon per oxidized luciferin.

Since the differences in luciferin substrate utilization result in no crossover reactions, both techniques, the D-luciferin system and the coelenterazine system, can be used in parallel, for example, in the same animal. Each luciferase has its own substrate specificity, characteristic wavelength of light emission, and its optimal parameters.

Firefly luciferase was cloned in 1985 for the first time. Only 3 years later, an assay was invented that made it possible to detect luciferase activity in mammalian cell lysates. The development of this assay became a useful tool in order to set up in vivo studies of gene regulation with luciferase activity.

One of the many advantages of luciferase is that it is a perfect marker for gene expression because there is no posttranslational modification. Additionally, the halftime of luciferase in vivo is approximately $2 \mathrm{~h}$ (Ignowski and Schaffer 2004). The light emission from this firefly catalyzed luciferin reaction is broad band from $530 \mathrm{~nm}$ to $640 \mathrm{~nm}$ and peaks at $562 \mathrm{~nm}$. The spectrum of this emission allows, together with the properties of biological tissues, the light to penetrate through several centimeters of tissue. This is true especially for light with spectral content of above $600 \mathrm{~nm}$. This special feature can be used to detect light that is emitted from internal organs in mice expressing luciferase as a reporter.

There are several factors involved affecting the sensitivity of detecting internal light sources. One major factor is the level of luciferase expression of cells. Another one is the depth of cells expressing luciferase within the body because it influences the distance of photons that have to travel through the animal tissue. Last is the sensitivity of the detection system used for collecting the light signal.

Another big advantage of this method is the commercial availability of luciferin or D-luciferin. Several biochemical companies all 
over the world sell it for research reasons (e.g., Life Technologies, Sigma-Aldrich, PerkinElmer, and many more).

For experimental handling, the first step in order to start a bioluminescence measurement is to place the animals in a dark chamber. To detect the light, emitted from the animal, photons are collected by a charge-coupled device (CCD) camera in combination with a computer for image acquisition and analysis of data. These CCD cameras first gather and then convert photons into electrons after striking silicon wafers. The intensity of incident photons is encoded into electrical charge patterns to generate an image.

During the last years, great advances have been made in order to improve detector technology to get better sensitivity and image quality. The background noise of the bioluminescence imaging machine is reduced by supercooling of the system and mounting the CCD camera in this lighttight dark box as mentioned before.

For the bioluminescence experiment itself, it is important that acquisition and analysis parameters remain constant when relative photons or counts are acquired. This includes, for example, the animal position relative to the CCD camera, the region of interest, and f-stop (the ration of lens focal length to the diameter of the entrance pupil). The absolute unit of radiance is photon/s/ $\mathrm{cm}^{2} /$ steradian, and it refers to the photons per second of light that radiate from the mouse in a unit area $\left(1 \mathrm{~cm}^{2}\right)$ and unit angle (1 steradian). This unit is more quantitative but enables an easier comparison of data in an experiment when image acquisition parameters are greatly different.

The concentration of administered luciferin also needs to be kept stable during experiments. Using the most commonly used concentration of $150 \mathrm{mg} / \mathrm{kg}$ per body weight for an animal, the experiment is cheap (with costs of around 300 Euro per $1 \mathrm{~g}$ D-luciferin), nontoxic for the laboratory animal, and easy to order and storable. The D-luciferin solved in PBS can be stored in- $20{ }^{\circ} \mathrm{C}$ for several months up to 1 year.

However, one problem for bioluminescence imaging is caused by D-luciferin itself. Since it is not produced by rodents, it needs to be injected systemically in order to travel through the bloodstream and needs to pass different membranes or barriers before it can reach the point or cells of interest. Different types of injections are discussed in the next chapter in more detail.

In addition, another disadvantage that D-luciferin causes itself is that it was first believed that for the rapidness of the enzyme luciferase, there is no restriction for the used D-luciferin. But it was reported that the detected in vivo signals correlate with the injected substrate dose, especially if there is a high number of cells expressing luciferase in vivo. This phenomenon shows that the higher the administered dose of D-luciferin in the animal is, the better the enzyme luciferase works close to its maximum velocity.

Because of the similarity of luciferin to albumin, it does not cause an immune response. Even though luciferin is able to freely cross the blood barrier and the placental barrier, toxicity appears low (Tiffen et al. 2010).

\subsubsection{Luciferase as a Reporter Gene for In Vivo Imaging}

The luciferase substrates need to be injected in rodents in order to be provided for the bioluminescence reaction in vivo as mentioned. There are different choices of injections that the experimenter can choose: intravenously and intraperitoneally are the most commonly used. Additionally, there are subcutaneous injections or less frequently used intratumoral injections or oral applications (Hiler et al. 2006; Inoue et al. 2009; Keyaerts et al. 2008, 2012; Wang and El-Deiry 2003). One advantage of intraperitoneally injections is the straightforwardness because the animal does not need to be anesthetized and the procedure itself is fast and easy. For injections in the tumor region, depending on the tissue or organ, or intravenously, the animal needs to be anesthetized. Before starting an experiment, several factors need to be considered. First, the injection site of the substrate needs to be discussed. This depends, for example, on the health situation of the animal and the question whether 
there is an incubation time needed before starting a bioluminescence measurement. It takes several minutes for the substrate to reach the region of interest (ROI); therefore, the times of incubation need to be validated for every experimental approach. The time that is needed for the luciferin to reach the ROI may differ from tissue to tissue and in every experimental setup. For example, the incubation time of the substrate can differ depending on the organ or tissue where cells expressing luciferase are placed: the abdomen, neck, or brain of the animal.

To give an example for bioluminescence imaging in a tumor model, one excellent example for the use of bioluminescence imaging in preclinical research is the detection of brain tumors in small rodents. D-luciferin readily crosses the blood-brain barrier and allows early examination of tumor growth in the brain tissue. Depending on the cell line and the strength of luciferase expression in the brain, tumor cells can be determined only hours after cell injection.

In addition to the time that is needed for the luciferin to cross the total body of the animal, the start point for the bioluminescence measurement needs to be determined because the bioluminescent signal proceeds over time. Imaging of the whole animal can be conducted 5-15 min after an intraperitoneally application of D-luciferin, with a relatively stable light emission level for 30-60 min, depending on the experimental conditions. Afterward the BLI signals decrease and disappear after a maximum duration of three hours.

The reaction in the animal emerges in no background signal for the D-luciferin reactions in the absence of the enzyme. For example, for the coelenterazine reactions, an auto-oxidation of this high-energy molecule occurs at a low velocity. This results in a low background signal even if there is no luciferase present. For in vitro luciferase assays, it is described that with boundlessness of ATP, oxygen, and D-luciferin, the correlation between enzyme concentration and signal intensity is excellent.

In contradistinction to advantages by using bioluminescence imaging, the interpretation of data obtained from in vivo bioluminescence is not always straightforward.
For example, a mouse bearing a massive subcutaneous tumor expressing luciferase leads to a bioluminescence signal after injection of luciferin. This signal can be misinterpreted. Cells in the core of the tumor can be apoptotic or parts of the tumor necrotic. Necrotic tissue is not able to express luciferase or to provide oxygen or ATP. This leads to a reduction of the total bioluminescence signal, and the signal is not correlated to the real size of the tumor. In addition, parts of the tumor region that grow deeper in the tissue can lose maximal values of counts because of the depth. The emitted counts do not display a correlation of tumor cells, and therefore it can result in an imbalance between signal and real tumor volume. Furthermore, the density of vessel density or the leakiness of vessels that means reachability of luciferin for the luciferase expressing cells needs to be kept in mind because it can influence the bioluminescence signal as well. This has to be considered for the measurement of growth rates of brain tumors, for example. Questions that can arise while working with brain tumors are if the luciferin is crossing the blood-brain barrier up to $100 \%$ or if the tumor region in the brain is reachable via new developed vessels. In general, a highly vascularized tumor, independent from the tissue, might even give a much higher bioluminescence signal than a tumor of the same size with less vessel density.

A major point that is important for the experimental setup and the interpretation of data is the approximately tenfold loss of photon intensity for each centimeter of tissue depth. Furthermore, images are surface weighted meaning that light sources closer to the surface of the animal appear brighter compared with deeper sources. In addition, changes in geometry can occur and can influence, for example, the growing tumor or scar tissue, or the optical properties of tissues can affect light scatter or absorption and therefore the detected bioluminescent signal.

In literature, there is one commonly used D-luciferin concentration described, $150 \mathrm{mg} / \mathrm{kg}$, that is nontoxic for the animal and used by many researchers. For coelenterazine, a much lower concentration of $0.5-4 \mathrm{mg} / \mathrm{kg}$ is usually injected because of a limitation of solubility of the substrate in aqueous solution. 
There are several parameters that can influence the sensitivity of bioluminescence imaging. Newly developed instruments, for example, with a new camera system are more sensitive in detection of small signals.

As mentioned before, the signal can be weakened by the depth of the tissue. Additionally the hair of the rodent can disturb or weaken the signals as well.

Parameters for imaging, like acquisition time, binning, and the positioning of the animal, need to be optimized for every experimental approach. Also of high importance for the bioluminescence experiment is the level of promoter activity controlling the expression of luciferase. In addition, the amount of luciferin injected in the animals and the diet are sensitive parameters as the gut phosphorescence leads to an increase in the background signal in animals.

But in contrast to other in vivo preclinical imaging techniques, bioluminescence imaging is a versatile and cost-effective technique with high sensitivity. Three to five animals can be measured in parallel at the same time during one experiment, and the acquisition of data takes a few seconds up to some minutes. By reducing the amount of animals that can be investigated in parallel during one measurement and by lowering the distance to the CCD camera, the sensitivity can be increased. Optimizing settings and conditions leads to light detection only from areas that contain luciferase, and there is no or minimal background noise visible.

One critical point in BLI is the half-life time of luciferase. The half-life of luciferase activity in live cells was measured in real time and was 2 h (Ignowski and Schaffer 2004).

The enzyme has to be produced continuously for the detection of signals. When cells, that express the luciferase, die or when the promoter elements turn the luciferase "off" or inactivate the gene signals from the luciferase, activity disappears.

In comparison to other in vivo imaging techniques, the measurement time for bioluminescence is significantly less (Zinn et al. 2008). Data acquisition with PET, SPECT, or MRI takes 30-60 min, and only one rodent can be measured during that time. Therefore, there is a limitation of animals that can be monitored at one single day during one experiment. In comparison, five mice can be measured in parallel in a bioluminescence experiment, and the whole measurement takes only some minutes.

This fact increases the amount of animals that can be measured at one single day. Additionally in comparison to PET, SPECT, or MRI, data analysis takes less time for BLI. In PET and SPECT, contrast agents are needed, and radiolabeled probes have to be prepared prior to the experiment. PET or SPECT result in background signals that reduce the sensitivity or signals in all locations, including those compounds that are metabolized or excreted by normal routes. Bioluminescence imaging can provide $2 \mathrm{D}$ and 3D images. But one major disadvantage of this method is the lack of translation to the application in humans because of the requirement for genetically encoded luciferase and the dependence of light signal on tissue depth.

\subsubsection{Functions of Bioluminescence}

The research that can be performed with bioluminescence imaging can be divided into two major parts. One includes routine investigations, in which a control element (promoter) for the luciferase that is always active in transfected cells is used. It is "on" under all conditions and in all tissue types. A highly active promoter that is often used and expressed in all tissue types is the cytomegalovirus (CMV) immediate-early promoter. Purposes for routine applications in vivo are detection of tumors or monitoring of their growth in cancer research. Gene therapy studies in combination with targeting of vectors or investigation of migration of adoptively transferred cells in cell therapy studies are also commonly performed.

For tumor imaging, plasmid or viral techniques are effective for the stable transfection of cancer cells with the luciferase reporter construct. This constitutive expression of luciferase is one strategy to use bioluminescence as a tool. These cells that constitutively express luciferase are 
implanted in syngeneic animals, but in the case of human cancer cell lines, immunodeficient mice or rats are required and can be monitored over a specific time.

Studies in combination with BLI have been reported for human cancer cell lines of meningioma, bladder cancer, prostate adenocarcinoma, neuroblastoma, hepatocellular carcinoma, breast cancer, hematologic malignancy, lung cancer, melanoma, and glioma models and moreover encompass humanized as well as metastatic models (Maes et al. 2009).

For example, bioluminescence showed an excellent correlation between tumor size and light emission when human breast cancer cells, a subclone of MDA-MB-231, that stably express the luciferase gene under control of the CMV promoter, were implanted in the mammary fat pad of a mouse model. By bioluminescence imaging, the cells were detected in the mammary fat pads of nude mice, and later, tumor growth rates could be determined. This method has been described for the preclinical assessment of the effectiveness of novel therapies for ovarian cancer and for animal models of prostate cancer (Brakenhielm et al. 2007; Kanerva et al. 2003). Differences in obtained bioluminescence signals correlate with a reduction in tumor size after treatment.

One study showed a tumor determination in the mammary fat pad of a mouse 19 days after injection, but with bioluminescence imaging, tumor cells $\left(1 \times 10^{6}\right)$ could be detected immediately after implantation. $15 \mathrm{~min}$ after intraperitoneal D-luciferin administration, the bioluminescent signal could be measured. The same study defined the minimum amount that could be detected subcutaneously by BLI with only $5 \times 10^{3}$ cells (Zinn et al. 2008). This example shows the sensitivity of this detection method, and one advantage the following: luciferase expressing tumor cells can be detected immediately after implantation in the animal model even weeks before the tumor can be detected by manual testing.

In comparison to monitoring a subcutaneous tumor that is visible or can be detected with manual testing, detection of metastasis of cancer cells within the whole body in rodents is not trivial. For this approach, bioluminescence imaging is particularly sensitive. Cowey et al. could show that immediately after injection of MDA-MB-435 breast cancer cells in the left ventricle of mouse hearts, the cells were detected in the blood pool with an overall body scan with BLI (Cowey et al. 2007). Forty-two days after injection of cells, bioluminescence imaging allowed the detection of metastases in the spine, joints, and head (mandibles).

For the detection of brain tumor growth in vivo, one of the best options besides MRI is bioluminescence imaging. The luciferin is able to cross the blood-brain barrier, and even days after tumor cell injection in the brain, signals can be measured. There are reports available that describe a detection of tumor cells even 7 days after injection in the brain.

In order to monitor the increasing luciferase expression and therefore increasing proliferation of human glioblastoma cells in a mouse model in vivo over time, 75,000 U87 MG cells, lentivirally transfected with luciferase, were injected in the right hemisphere of mouse brains according to Sabag, Bode, and Fink et al. (Sabag et al. 2012). The first bioluminescence signal was measured 12 days postinjection. Figure 16.8 shows a representative image of a measurement of mice each bearing a glioblastoma multiforme. The expression of luciferase in the brain was detected after an incubation time of ten minutes after intraperitoneal injection of D-luciferin $(150 \mathrm{mg} /$ $\mathrm{kg} \mathrm{KG}$ ), exposure time of seven minutes, and binning of four. No expression of luciferase was detected initially after injection of PBS in a tumor-bearing mouse (negative control, right mouse).

Injection of D-luciferin resulted in bioluminescence signals in the here shown two mice. Both mice were injected with the same amount of tumor cells at the same day.

The obtained bioluminescence signals demonstrate the heterogeneity of glioblastoma growth in vivo. Mice were then chosen for MRI studies using a newly developed contrast agent for the detection of brain tumors in mouse models in vivo. The goal was to investigate the enrichment 

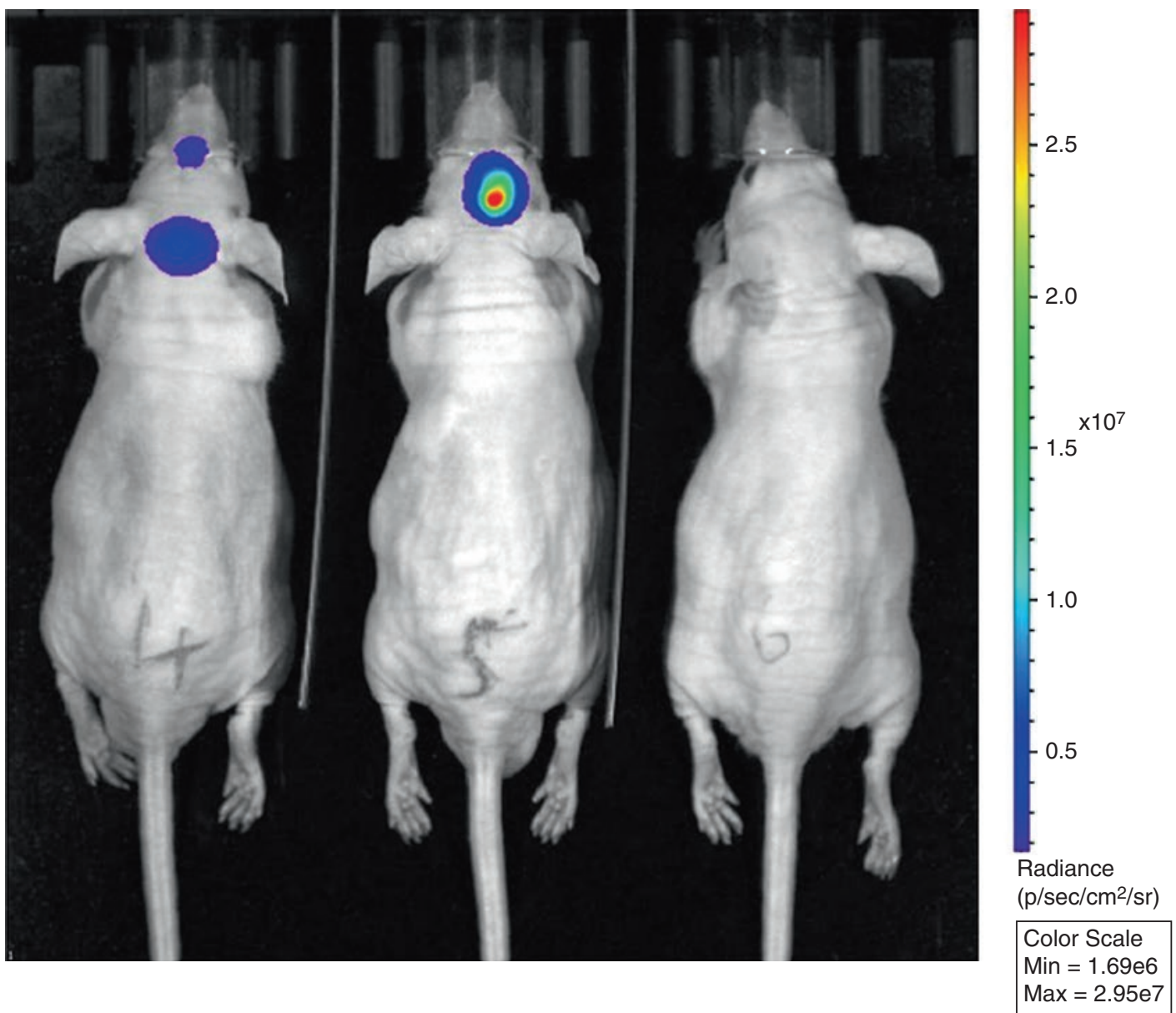

Fig. 16.8 Bioluminescence imaging of nude mice 12 days after injection of 75,000 luciferase-positive U87 MG glioblastoma multiforme cells into the right hemisphere. (Left, middle) Luciferin $150 \mathrm{mg} / \mathrm{kg}$ bw was given intraperitoneally; imaging times of $7 \mathrm{~min}$ and a binning of 4

of contrast agent in brain tumors with different sizes in order to optimize the resolution of MRI and to find a correlation with obtained bioluminescence signals.

One disadvantage that we observed during this study was the deviation from the location of the tumor in the brain and the obtained bioluminescence signal. A tumor grown deeper in the brain tissue led to a smaller BLI signal compared to a tumor, smaller in size, that grew in a more cranial direction. The method is not suitable for monitoring treatment effects of brain tumor growth in vivo. The reason is the event of default of missing the right time of, for example, injection were kept constantly A brain tumor-bearing mouse as control after injection of PBS shows no BLI signal (right). The pseudo-color scale bar represents the intensity of light emission with different colors (Unpublished data: T. Krüwel and J. Bode)

of a drug. The brain tumor is only detectable with BLI when (i) the size is big enough and (ii) the location is not too deep in the tissue.

One advantage of this technique is the fast and easy way of monitoring the proliferation of cells over time in vivo depending on the location of tumor cells in the tissue. It is a technique that allows to investigate tumor growth of a cancer type that cannot determined easily in vivo.

Noninvasive bioluminescence imaging was also applied to monitor preclinically pancreatic tumors, a tumor also not visible from the outside. Therefore, $1 \times 10^{6}$ luciferase-transfected human pancreatic adenocarcinoma PancTu-I cells 
(PancTu-I-luc) were orthotopically injected into the head of the pancreas of nude mice. Wholebody scans 23 days after cell implantation using the IVIS Spectrum in vivo imaging system (Fig. 16.9) and $13 \mathrm{~min}$ after the i.p. injection of $150 \mathrm{mg} / \mathrm{kg}$ D-luciferin show bioluminescence signals over the tumor areas corresponding to a distinct tumor growth and expansion in each mouse.

Another example for an application of bioluminescence imaging is the detection of bacteria (Burns-Guydish et al. 2005; Siragusa et al. 1999), for example, the colonization of Citrobacter rodentium in wild-type C57BL/6 mice. It was shown that the bacterial colonization of the ani- mal after inoculation could be monitored over a time of over 15 days. The technique demonstrated that the pathogens are cleared from the body at day 9 or 10 post transfection that was complete by days 15 to 17 . By applying a bioluminescent strain of $C$. rodentium, it could be shown that after the removal of the abdominal wall and bacterial exposure of the cecum and colon, the Citrobacter preferentially colonized the cecal lymphoid patch and the mid and distal colon. The mice were kept in a chamber in a biosafety cabinet. The chamber was sealed to avoid bacterial exposure of other animals; therefore, bioluminescence signals and the position of bac-
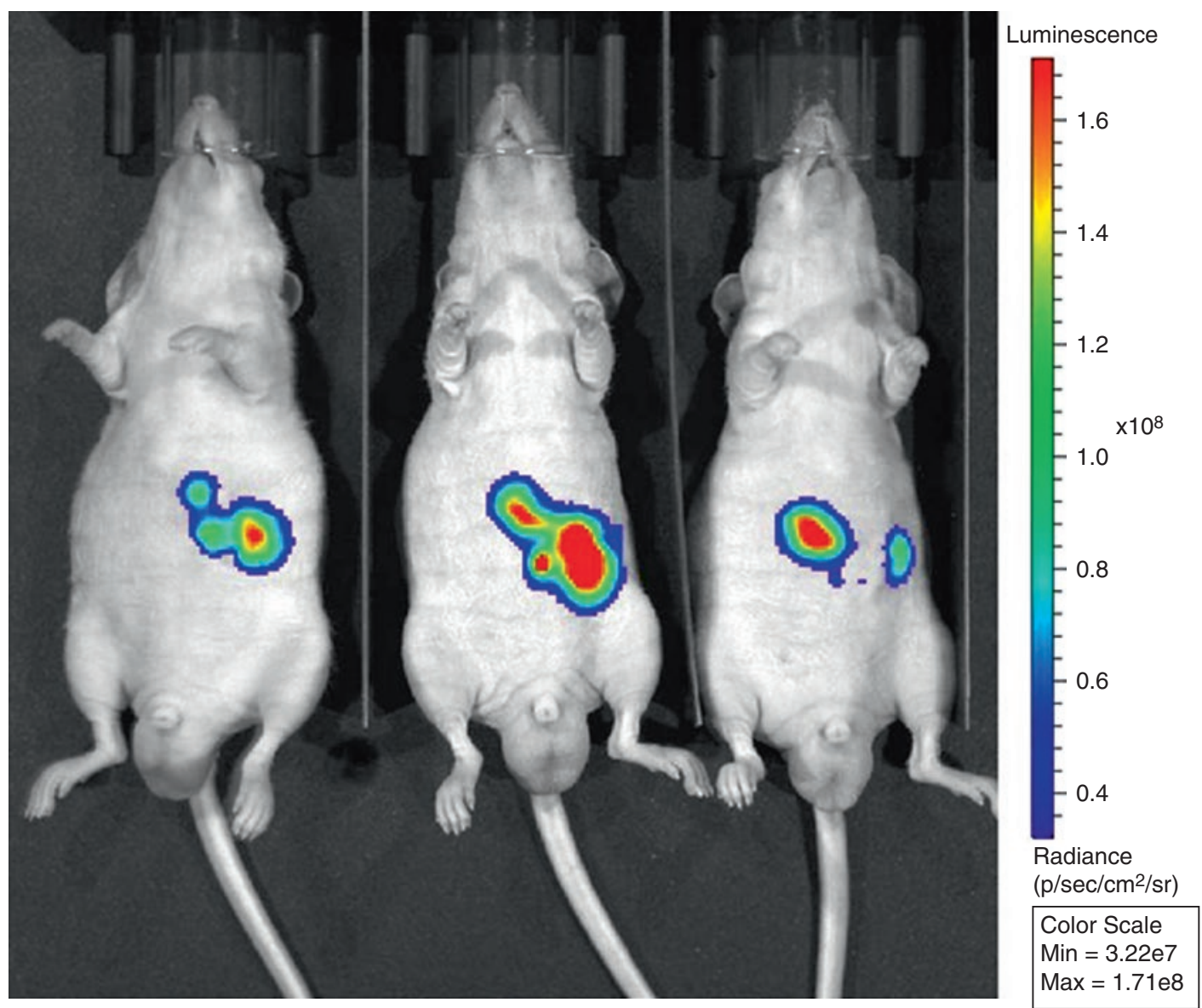

Fig. 16.9 Representative in vivo 2D bioluminescent images of three pancreatic tumor-bearing nude mice, 23 days after orthotopic implantation of $1 \times 10^{6}$ luciferasetransfected PancTu-I cells into the head of the pancreas.
Bioluminescence signal was measured 13 min after i.p. injection of D-luciferin $150 \mathrm{mg} / \mathrm{kg}$ (Unpublished data: M. Saccomano) 
teria in the animal were sensitively detected (Wiles et al. 2004, 2006).

There are specialized research applications for reporter constructs which are only active under defined conditions. For example, detection of apoptosis with bioluminescence signals can be realized to evaluate newly developed cancer therapies. In order to unravel gene regulation or to study other biological processes, one approach using bioluminescence imaging is to generate transgenic mice, expressing luciferase during developmental processes.

In another transgenic mouse model, firefly luciferase was expressed under the control of the VEGFR2 promoter in order to noninvasively elucidate the temporal profile of VEGFR2 expression after stroke as a biomarker for VEGF/ VEGFR2 signaling (Adamczak et al. 2014). In their study they described that VEGFR2-related signaling is active at least up to 2 weeks after the infarct and results in increased vascular volume. Furthermore, this study presented a novel strategy for the noninvasive evaluation of angiogenesisbased therapies.

Bioluminescence imaging detects the expression of the luciferase as a genetic reporter. There are several opportunities to control the expression based on the activity of the promoter element, for example, monitoring the expression of luciferase in the liver under specific tissue conditions. The CMV promoter is only "on" or active when it is integrated in hepatocytes. A different promoter like cyclooxygenase $2 \mathrm{~L}$ (cox-2 L) is not active under normal conditions in the liver, but it is active or "on" in the presence of inflammation (Ishikawa et al. 2006). It was shown that the technique is sensitive enough to detect the inflammation caused after lipopolysaccharide (LPS) injection. After LPS injection, expression of luciferase was induced and could be measured. No expression in liver tissue was detected initially (Iyer et al. 2005, 2006).

Another well-characterized application of bioluminescence imaging is the investigation of tumor hypoxia.

Tumor hypoxia plays an important role in promoting malignant progression and affecting ther- apeutic response negatively. There is little knowledge about in situ, in vivo, tumor hypoxia during intracranial development of malignant brain tumors because of lack of efficient means to monitor it in these deep-seated orthotopic tumors. Bioluminescence imaging with a reporter gene system under the control of a promoter sequence can be used to monitor hypoxic stress in a noninvasive way. Under hypoxic stress, signaling responses are mediated mainly via the hypoxiainducible factor- $1 \alpha$ (HIF-1 $\alpha)$ to drive transcription of various genes. For example, Saha et al. used a HIF- $1 \alpha$ reporter construct, 5HRE-ODDluc, stably transfected into human breast cancer MDA-MB231 cells (MDA-MB231/5HRE-ODDluc) to study tumor hypoxia. In vitro, a HIF- $1 \alpha$ bioluminescence assay was performed by incubating the transfected cells in a hypoxic chamber $\left(0.1 \% \mathrm{O}_{2}\right)$ for $24 \mathrm{~h}$ before the bioluminescence signal measurement, while the cells in normoxia $\left(21 \% \mathrm{O}_{2}\right)$ served as a control. Significantly higher photon flux observed for the cells under hypoxia suggests an increased HIF- $1 \alpha$ binding to its promoter (HRE elements), as compared to those in normoxia. Cells were injected directly into the mouse brain to mimic a breast cancer brain metastasis model. In this model, in vivo bioluminescence imaging of tumor hypoxia dynamics is initiated 2 weeks after implantation and repeated once a week. Bioluminescence reveals increasing light signals from the brain as the tumor progresses, indicating a rise in intracranial tumor hypoxia (Saha et al. 2011).

In order to control luciferase expression with the help of promoter elements, one approach is to synthesize an inactive luciferase enzyme by linking a cleavable protein subunit to luciferase. For example, specific sequences within the subunit are activated by caspase 3 . The caspase activates the luciferase, and this is one way to monitor apoptosis in vivo (Laxman et al. 2002). The application of this reporter in combination with bioluminescence imaging was used as an early detector of apoptosis (Rehemtulla et al. 2004), for example, in response to therapy.

A recently published paper by CruzMonserrate et al. showed that bioluminescence 
imaging can be applied knowing that tumor cells and the surrounding tissue have different $\mathrm{pH}$ values. They utilized ph-sensitive probes determining primary tumors and metastasis of human pancreatic cancer xenografts (Cruz-Monserrate et al. 2014). They applied bioluminescence in order to validate signals from fluorescencelabeled insertion peptides they used for the localization of primary tumor and metastases in this particular mouse model.

Paulmurugan et al. described an inactive luciferase enzyme that responded to a drug to form an active luciferase under conditions of specific protein-protein interactions. This approach can be used to track interactions between proteins in living animals or cells (Paulmurugan et al. 2002).

The generation of transgenic mice, for example, can be established as a tool to track naïve T-cell populations in real time (Chewning et al. 2009). This is only one of the examples of transgenic mice with luciferase constructs that are available in academic research.

In this approach, the firefly luciferase gene was cloned into human cd 2 genes, limiting the constitutive expression to all T-cells. Sorted cell populations clearly gave evidence for the T-cellspecific expression in CD4+ and CD8+ T-cells. In vivo this approach was deployed to image intact delineation of primary and secondary lymphoid organs.

\subsubsection{Conclusions}

There are several parameters that influence the sensitivity of bioluminescence imaging.

The most important determinants are:

- Instrumentation

- Hair

- Depth in tissue

- Imaging parameters (acquisition time, binning, animal position)

- Level of promoter activity controlling the luciferase expression
- Amount of luciferase substrate injection

- Diet

It is essential to optimize these parameters for each experiment. Of primary importance are especially the CCD camera and a position of the animal close to the camera. Together with longer acquisition times and higher binning, the sensitivity of the measurement can be increased.

Bioluminescence imaging is in summary a versatile and cost-effective technology that has high sensitivity and efficiency. Up to five mice can be imaged at the same time with acquisition times ranging from some seconds to some minutes. That means it is possible to image up to 150 mice in a single day if needed. By decreasing the number of mice in one measurement, the sensitivity can be increased. A simple ROI analyses can easily reduce the data.

Using optimal conditions for an experiment, light is only detected from areas that contain luciferase, and there is minimal or no background noise.

Since luciferase has a short biological halflife, it must be continuously produced for the measurement of signals. In the case of death of cells, the signal is lost. In a similar way, changes in luciferase levels can be discovered when promoter elements driving luciferase are turned off or on.

The majority of bioluminescence experiments take advantage of the well-characterized firefly luciferase or Renilla luciferase proteins, and when used in conjunction with alternate imaging technologies, they can provide extremely thorough and sophisticated datasets.

In comparison to other imaging systems such as SPECT, PET, or MRI, the cost of bioluminescence imaging systems is significantly less.

In future, an advancement of this method can lead to a higher sensitivity of tumor cell detection in animal models. Further developments could help to detect small metastasis and cell clusters in order to start treatments of diseases as soon as possible. 


\subsection{Optical Coherence Tomography (OCT)}

Martin Hofmann, Volker Jaedicke, Peter Cimalla, and Edmund Koch

\subsubsection{Tissue Properties and Ballistic Photons}

Optical imaging of biological tissue suffers from the strong attenuation of light in the tissue. Therefore, standard optical imaging is limited to the tissue surface or to transparent tissue, for example, the eye. However, under certain circumstances, the attenuation is mostly not due to absorption but due to scattering of light. This enables advanced optical imaging technologies like optical coherence tomography which allows looking into biomedical tissue.

In more detail, the absorption coefficient and the scattering coefficient of tissue are shown as a function of wavelength in Fig. 16.10 (Roggan et al. 1999). The absorption coefficient drops from $300 \mathrm{~nm}$ to a minimum at $1100 \mathrm{~nm}$ and then rises again. The scattering coefficient exhibits a weaker wavelength dependence and slightly decreases from $300 \mathrm{~nm}$ to $2100 \mathrm{~nm}$. But obviously, the scattering coefficient in the wavelength regime between 600 and $1300 \mathrm{~nm}$ is about two orders of magnitude higher than the absorption coefficient. Accordingly, light attenuation in tissue in this wavelength range is mostly due to scattering, and absorption plays a minor role. That is why this spectral range is also called "optical window."

As a consequence of these optical tissue properties, approaches for optical imaging in tissue have to operate in this optical window and have to make use of concepts to suppress or circumvent scattering.

To motivate the strategy used in OCT, we now look schematically at the scattering processes. This is shown schematically in Fig. 16.11.

All inhomogeneities inside the tissue represent potential scattering centers as indicated by the black dots in the schematic picture in Fig. 16.11. When photons of light enter the tissue, there is a small but finite probability that they propagate through the tissue without any scattering event. Of course, this probability drops strongly with increasing tissue thickness and with increasing density of scatterers. For practical biomedical tissue, the maximum path length or the penetration depth for these unscattered socalled ballistic photons (Yoo and Alfano 1990) is below $1 \mathrm{~mm}$. More probably, the incoming photons will be multiply scattered before they leave the tissue. In between these extremes, there are also a few photons which are only weakly scattered. These are sometimes called "snakelike photons"(Yoo and Alfano 1990).
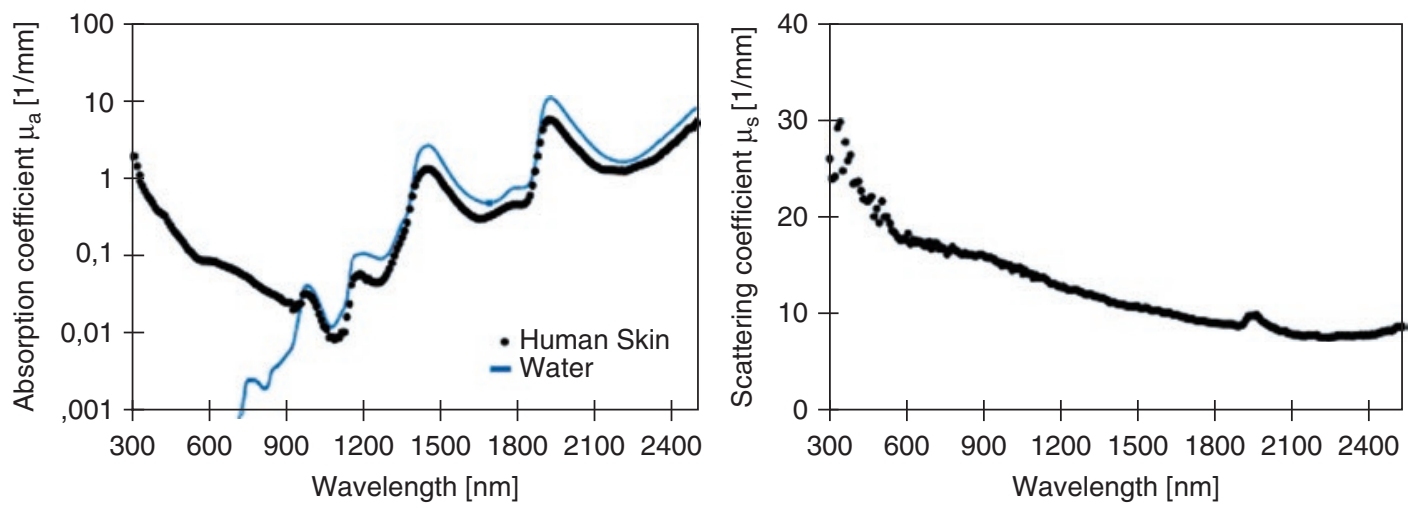

Fig. 16.10 Absorption coefficient and scattering coefficient of biomedical tissue as a function of wavelength (Roggan et al. 1999) 


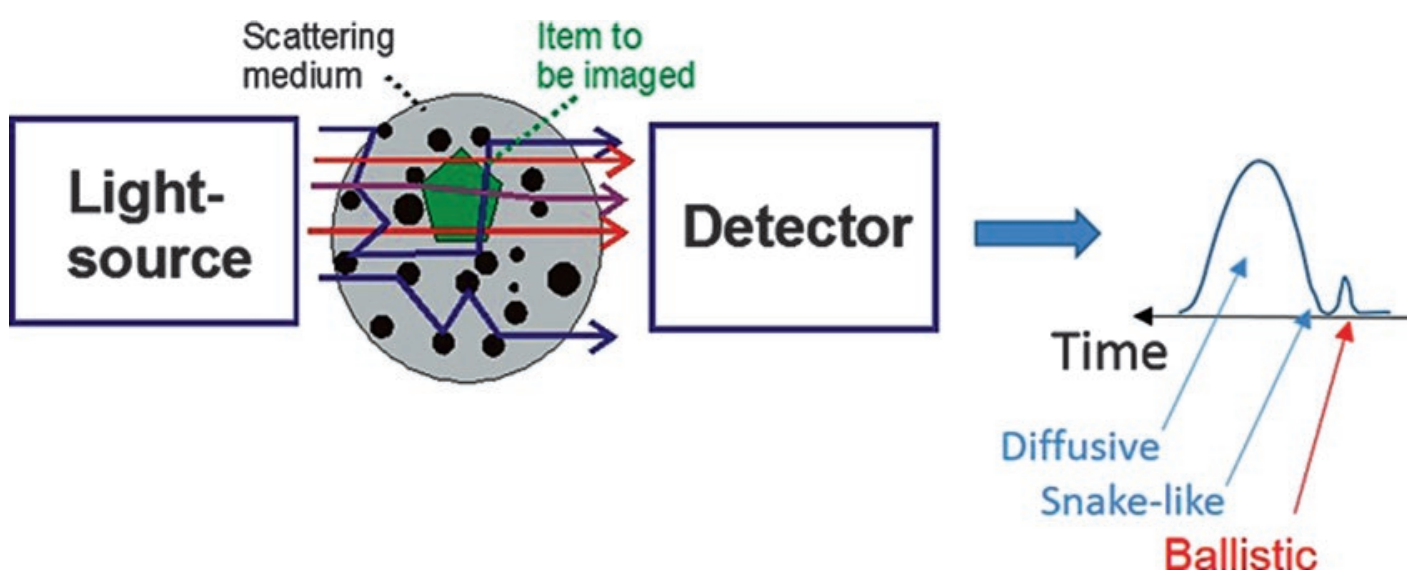

Fig. 16.11 Light scattering in tissue

If one aims to recover an image of the tissue from the transmitted photons, it is clear that the ballistic photons would contain the full image information. In contrast, the scattered photons would carry no image information anymore because of their arbitrary statistically distributed propagation paths. The snakelike photons may in some cases still contain some smeared-out image information.

Unfortunately, due to the high scattering coefficient, the transmitted light will mainly contain scattered photons. Any image information encoded in the ballistic photons is thus invisible by eye or with a standard camera because it is superimposed by a more or less unstructured background of scattered photons.

Accordingly, image concepts should be developed that separate the ballistic photons from the scattered ones or even suppress the scattered photons. Conceptually, one option is based on the fact that ballistic photons have a considerably shorter propagation path from the light source through the tissue to the detector than the multiply scattered photons. Accordingly, the ballistic photons have a much faster propagation time. If a pulsed light source is used, the ballistic photons might be filtered out with a fast enough camera that switches off after the arrival of the ballistic photons (Hee et al. 1993; Wang et al. 1991). But in practice, this approach suffers from enormous difficulties. For a clear separation of the ballistic photons, the light source should provide pulses considerably shorter than a picosecond $\left(10^{-12} \mathrm{~s}\right)$, and the camera should detect only in a sub-picosecond time window. Though nonlinear optical schemes have been demonstrated to enable detection on such shorttime scales (Wang et al. 1991), one fundamental problem cannot be overcome: the ultrashort pulses are substantially broadened in the tissue due to dispersion (i.e., wavelength dependence of the refractive index). Thus, the required temporal separation of the ballistic photons would not operate sufficiently in real biomedical tissue. Therefore, other separation schemes have to be implemented. In OCT, the coherence properties are used for separation of the ballistic photons (Huang et al. 1991). Before introducing the concept used in OCT, we first discuss the coherence of light sources.

\subsubsection{Coherence, Interferometry, and Diffraction}

Light is an electromagnetic wave. If its wavelength is exactly defined, the extension of this wave is infinite as indicated in Fig. 16.12a. In this case, the light is perfectly coherent that means all parts of the infinitely extended plane wave have well-defined phase relations to each other. Light from practical sources includes statistically distributed phase jumps as indicated in Fig. 16.12b.

In this case, the perfect phase relation of different parts of the wave is only maintained for the intervals in between two phase jumps. Accordingly, the perfect coherence is lost. To 
Fig. 16.12 $E(t)$ in a light wave with high (top) and low (bottom) temporal coherence

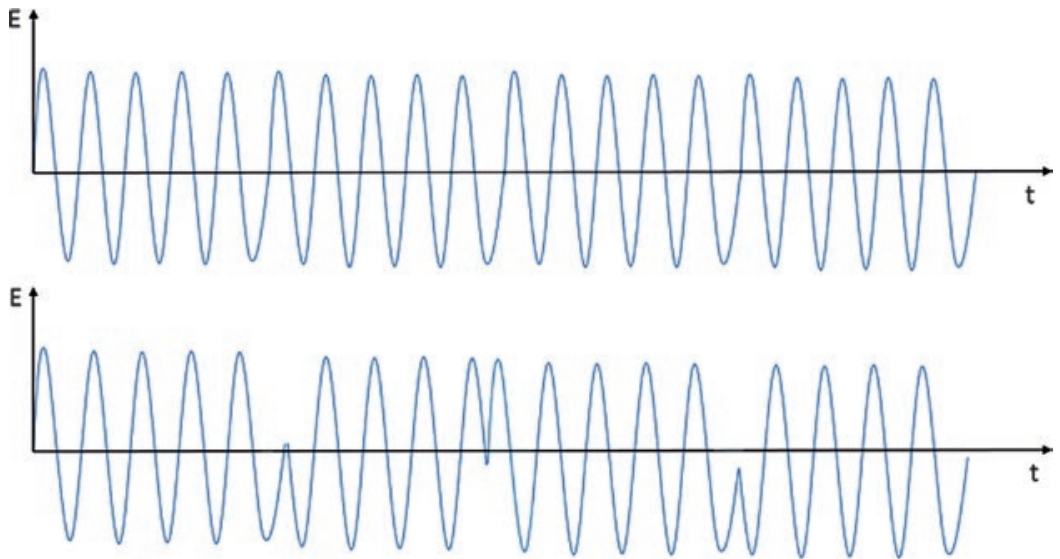

quantify the coherence, one defines the coherence length $l_{\mathrm{c}}$ of a light source as the average path length in between two phase jumps (Wolf 2007). Since light propagates with the speed of light c, the coherence length $l_{\mathrm{c}}$ is equivalent to a coherence time $t_{\mathrm{c}}$ given by $t_{\mathrm{c}}=l_{\mathrm{c}} / \mathrm{c}$.

The coherence length of a light source is directly linked to its spectral distribution. As mentioned above, an ideal, perfectly coherent light source $\left(l_{\mathrm{c}}=\infty\right)$ consists of only one wavelength. For realistic light sources, the coherence length is finite and inversely proportional to the spectral width following $l_{c}=0.44 \frac{\lambda_{0}^{2}}{\Delta \lambda}$

(Wolf

2007) for a Gaussian-shaped spectrum with center wavelength $\lambda_{0}$ and spectral width $\Delta \lambda$.

Coherence is usually measured by autocorrelating the light source with itself in an interferometer arrangement, for example, a Michelson interferometer (Michelson 1881).

In a Michelson interferometer, a light beam is split into two parts with a beam splitter. The two parts propagate toward a mirror or retroreflector and are backreflected, recombined, and superimposed on a detector. The detector measures the time-integrated intensity of the superimposed light fields. One of the two interferometer arms can be changed in length, e.g., with a moveable mechanical stage. That means, the path length difference $\Delta z$ or the time delay $\tau$ of the two arms can be varied. An interferogram corresponds to the signal $I(t)$ detected by the detector as a function of the delay time $\tau$. When $I_{1}$ and $I_{2}$ are the intensities in the two arms, $I(t)$ is given by

$$
I(\Delta z)=I_{1}+I_{2}+2 \sqrt{I_{1} I_{2}}|\gamma(\Delta z)| \cos \frac{2 \pi \Delta z}{\lambda},
$$

$\gamma(\Delta z)$ being the coherence degree as a function of path delay (Hering and Martin 2006).

While $I_{1}$ and $I_{2}$ are independent of $\Delta z$, the third term that depends on $\Delta z$ is called the interference term. Interference occurs when the two superimposed fields have a defined phase relation. If there is no phase relation, the timeintegrated superposition averages out to zero, and the interference term disappears. In other words, an interference term is only detected if the path length difference between the two interferometer arms is shorter than the coherence length $l_{\mathrm{c}}$. Thus, by measuring the interference signal as a function of $\Delta z$, one can determine the coherence length which is half the full width at half maximum of the coherence degree $\gamma(\Delta z)$.

So far, we have discussed the coherence of a light source in the propagation direction. This is usually called "temporal coherence" (Hecht 2005). In contrast, "spatial coherence" refers to the coherence in lateral direction (Hecht 2005). A light source with perfect spatial coherence exhibits no phase jumps along the lateral direction, i.e., perpendicular to the propagation direction. Spatially incoherent light sources are typically extended emitters, for example, standard lightemitting diodes with weak correlations between adjacent emission areas.

The important consequence for OCT is that the interference properties of the light source may be used to filter out the ballistic photons car- 
rying the image information. In particular, using light with a short coherence time or coherence length is advantageous (Huang et al. 1991). Let us consider to replace the short pulse light source in Fig. 16.11 by a light source with a short coherence length and to position the sample into one arm of an interferometer. Still, the ballistic photons have the shortest propagation path, and the multiply scattered photons have much longer propagation paths and require more time through the tissue. If the time difference between ballistic photons and scattered photons is larger than the coherence time, the interferometer can be moved to a position at which the ballistic photons contribute to the interference signal, while the scattered photons do not. This concept referred to as coherence filtering is the fundamental idea of optical coherence tomography (Huang et al. 1991). Its practical implementation will be discussed in the following section.

Before, let us briefly discuss what determines the spatial resolution in OCT. The coherence filtering implies that the resolution in propagation direction is determined by the coherence length of the light source. In a transmission geometry, the resolution would directly be given by the coherence length, while in the conventionally used reflection geometry, the resolution $\Delta_{\mathrm{z}}$ in propagation direction or axial direction is given by $\Delta_{z}=l_{\mathrm{c}} / 2$. In OCT, the resolution in axial direction is decoupled from the resolution $\Delta_{x y}$ in lateral direction. Like in conventional optical imaging systems, the resolution $\Delta_{x y}$ in lateral direction, i.e., the minimum distance at which two objects can be separated, is given by Abbe's diffraction limit $\Delta_{x v}=\frac{\lambda}{2 n \sin \alpha}=\frac{\lambda}{2 \mathrm{NA}}, \lambda$ being the wavelength of illumination, $n$ the refractive index, $\alpha$ half the aperture angle of the optics, and NA the numerical aperture of the optics (Abbe 1873).

In practice, standard OCT systems provide axial resolutions in the range of a few $\mu \mathrm{m}$, and their design usually provides similar values for the lateral resolution (Schmitt 1999) (Fig. 16.13).

In comparison to established nonoptical biomedical tomographic imaging concepts, OCT thus provides a uniquely high spatial resolution, while the penetration depth is significantly lower than for ultrasound imaging, magnetic resonance

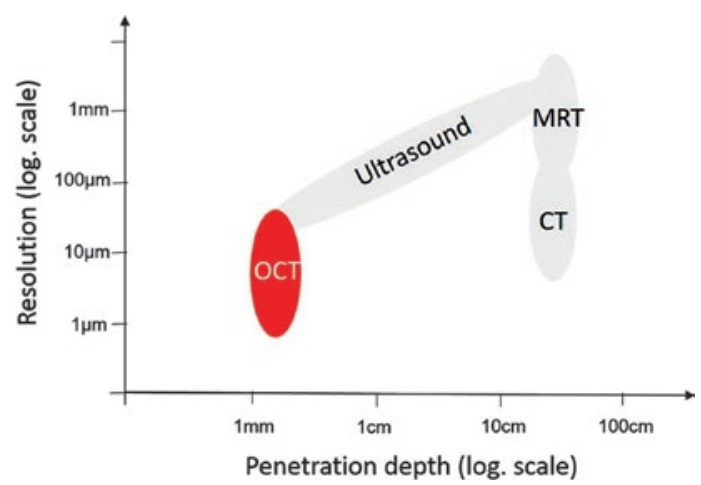

Fig. 16.13 Resolution versus penetration depth for OCT in comparison to ultrasound imaging, MRT imaging, and CT imaging

tomographic imaging (MRT), or computer tomography $(\mathrm{CT})$ based on X-ray imaging.

\subsubsection{Fundamental Principles of Optical Coherence Tomography}

Practical OCT systems are based on interferometers, typically on Michelson interferometers (Huang et al. 1991), but interferometers with different beam splitters for dividing and combining the beams allow higher flexibility and may use both rays from the second beam splitter, for instance, for differential detection. The various OCT concepts usually differ in the way the interferograms are recorded. The two main categories are time domain OCT (TD-OCT) (Huang et al. 1991) and frequency domain OCT (FD-OCT) sometimes called Fourier domain OCT (Leitgeb et al. 2003a). Both are schematically shown in Fig. 16.14

\subsubsection{Time Domain OCT}

A TD-OCT system consists of 3 main components: a broadband light source, the interferometer with sample arm and moveable reference arm, and the detector. As the broadband light source, one may use femtosecond laser systems (Morgner et al. 2000), white light continuum sources (Hartl et al. 2001), or superluminescent light-emitting diodes (LEDs) (Bayleyegn et al. 2012). Femtosecond lasers and white light continuum sources offer extremely broad spectra in excess of a few hundred nanometers (nm) and thus 


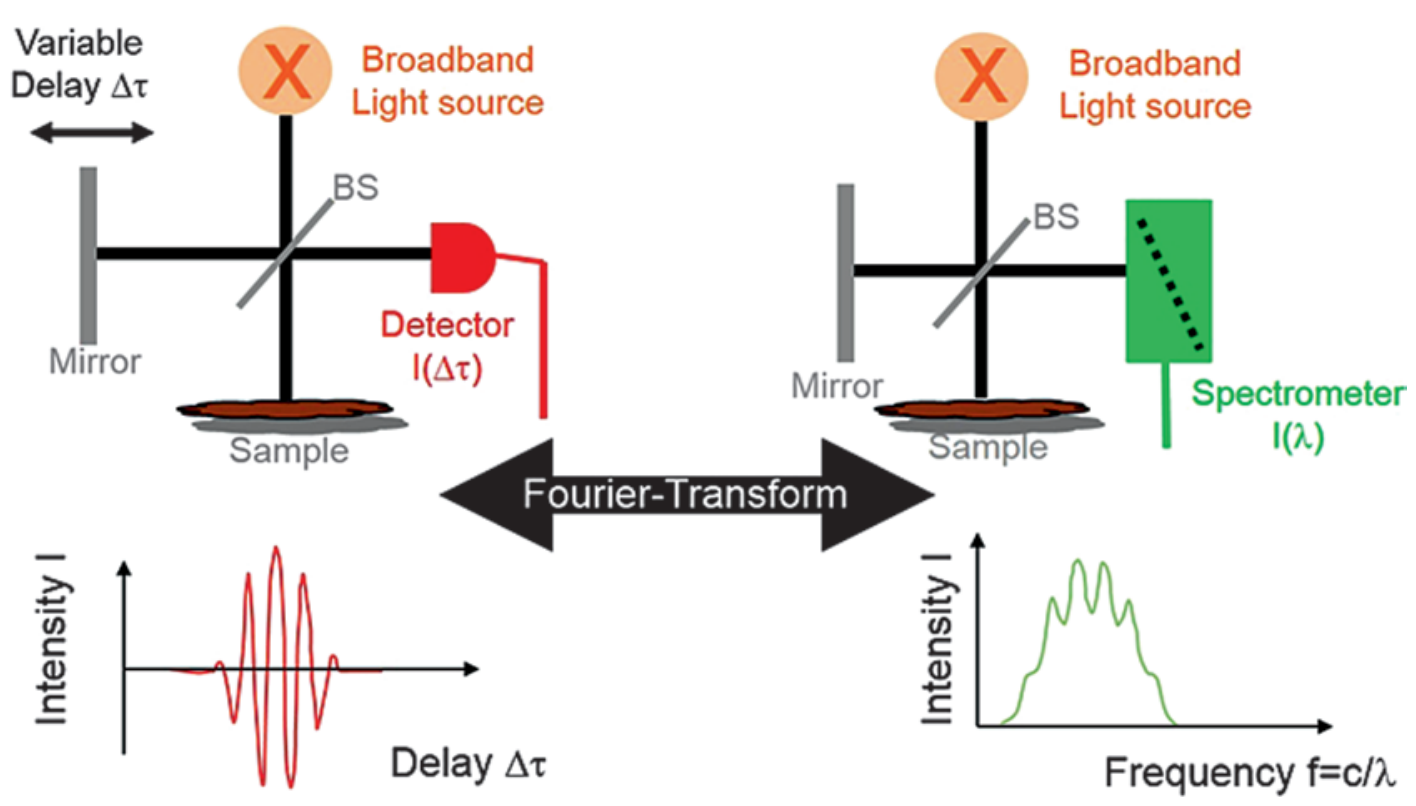

Fig. 16.14 TD-OCT (left) and FD-OCT (right). BS: beam splitter

enable axial resolutions down to the very few micrometer $(\mu \mathrm{m})$ range (Bouma and Tearney 2002). But these systems are expensive and complex. In contrast, superluminescent LEDs provide spectral bandwidths of several tens of nm up to about $100 \mathrm{~nm}$ (Bayleyegn et al. 2012) and are much more compact and cost-effective than the other sources. Thus, most standard OCT systems use superluminescent LEDs as light sources.

The output beam of the broadband light source is coupled into the interferometer where a beam splitter divides it into two parts. One part propagates to the sample under investigation where it is backreflected and then it propagates back and is directed onto the detector. The other part of the beam propagates into the reference arm which contains a mirror or retroreflector on a moveable delay stage. After reflection, it propagates back via the beam splitter and is also directed to the detector where it interferes with the beam from the sample arm (Huang et al. 1991).

The detector is a standard photodetector in the easiest realization. Alternatively, photomultipliers or avalanche photodiodes may be used to increase sensitivity.

An interferogram is generated when the interference signal is measured as a function of the time delay $\Delta t$ between reference arm and sample arm. When we consider that the sample is partially transparent and contains multiple reflecting interfaces inside, then the reflections of interfaces at different depths will appear at different times. Only when the propagation time to the corresponding interface and back corresponds within the coherence time of the light source to the propagation time through the reference arm, the backreflection from that interface will contribute to the interference signal. In other words, when the length of the reference arm is changed, only light reflected back from the sample that had the same propagation path length as that through the reference arm contributes to the interferogram. Accordingly, the position of the reference arm addresses a certain depth in the sample, and the strength of the interferogram at this position is a measure of the backreflection at that certain depth. In particular, interfaces between different substances in the sample will create stronger backreflections, so-called echoes of light (Drexler and Fujimoto 2008). Recording the strength of these echoes, i.e., the amplitude of the interference signal as a function of delay time or path length difference in the interferometer, provides a one-dimensional depth scan into the sample or a so-called A-scan (Drexler and Fujimoto 2008). In practical systems, the scanning of the reference 
arm induces a Doppler shift in frequency which is used in the signal detection scheme (Drexler and Fujimoto 2008).

For a two-dimensional (so-called B-scan) or three-dimensional image, mechanical scanning in the two directions perpendicular to the axial direction is required. This might be done with mechanical delay stages or, more practically, with galvanometer scanners (Duma et al. 2011) and microelectromechanical systems (Sun et al. 2010).

A disadvantage of TD-OCT systems is the limited speed, specifying the number of A-scans per second. The speed is mostly limited by the mechanical scanning of the delay line (Kim and Kim 2010). Many efforts have been made by using multiple reflections, piezoelectric-driven fiber-optic delay lines, and more. The fastest systems achieve A-scan rates of a few $\mathrm{kHz}$ with rapid scanning optical delay lines (RSOD) using resonantly driven mirror systems (Gao 2007).

\subsubsection{Frequency Domain OCT}

The interference signal represents a correlation of the fields from the reference and from the sample arm. According to the Wiener-Khintchine theorem, the Fourier transform of this correlation provides the spectral power density (Goodman 1985). In other words, the interferogram and, correspondingly, an A-scan can also be obtained when the spectrum of the interference signal is recorded and then Fourier transformed. This is the principle of Frequency domain or Fourier domain OCT which is also referred to as spectral radar (Bail et al. 1996). Another explanation is that for each wavelength, interference occurs independent of the path difference, and the phase of the interference depends on the product of path difference and wave number (reciprocal value of the wavelength) of the light. One advantage of FD-OCT over TD-OCT is that no movement of a mirror in the interferometer arm is required. On the other hand, FD-OCT requires spectrally resolved detection. This can either be done with a spectrally resolving detector or with a spectrally scanning source (so-called swept source OCT) (Chinn et al. 1997).

In the first case, the spectrometer is mostly realized by a grating for dispersion and a line array detector for simultaneous detection of the different spectral components (Bail et al. 1996). These systems are often called spectral domain OCT (SD-OCT). For systems operating in the near-infrared range below $1 \mu \mathrm{m}$ wavelength, this concept is well established because cost-effective line array detectors based on silicon technology are available. For wavelengths considerably above $1 \mu \mathrm{m}$, the silicon detector arrays have to be replaced by InGaAs detectors which are much more expensive.

Costly InGaAs line detectors can be avoided in the second case. In a swept source OCT system, also called optical frequency domain imaging (OFDI), one or two (in the case of differential detection) integrating detectors are used, and the source wavelength varies over time (Chinn et al. 1997). So the different wavelengths are detected sequentially, and no spectral resolution of the detector is required. Recently, extremely fast tunable swept sources have been demonstrated allowing up to 20 million A-scans per second enabling real-time 3D OCT imaging also called 4D imaging (Gora et al. 2009; Kottig et al. 2012; Wieser et al. 2010). Today's typical FD-OCT systems using commercially available swept sources or fast line scan cameras achieve A-scan rates in the range of $100 \mathrm{kHz}$.

While TD-OCT and FD-OCT systems generally provide similar physical information about the sample, FD-OCT systems have been shown to be more sensitive by a factor that scales with the square root of the number of spectral points (Choma et al. 2003; de Boer et al. 2003; Leitgeb et al. 2003a). To enable a fast processing of the data, the Fourier transformation is generally implemented as a FFT. Because neither the grating spectrometer nor the tunable source usually provides data linear in wave number, linearization of the data, often combined with oversampling, is applied before the FFT (Vergnole et al. 2010). For spectrometer-based systems of medium bandwidth, a combination of grating and prism can be used for linearization of the spectrum instead (Hu and Rollins 2007; Traub 1990). A further step in processing FD-OCT data is called spectral shaping. As the spectrum of the light source in combination with the sensitivity of 
the detector and the spectral transmission of the interferometer is neither flat nor Gaussian, spectral shaping is used to produce a spectral form of the interference that leads to moderate artifacts after the FFT. Typical window functions used are Hanning and truncated Gaussian (Eigenwillig et al. 2009).

While TD-OCT detects only the interference between light from the reference arm and the sample, FD-OCT also detects the interference between different reflections within the sample arm or other unintended reflections in the optical setup. This can lead to ghost images, often caused by the strong Fresnel reflex of the first sample surface, and fixed pattern structures. Such artifacts can be suppressed by implementing enough dispersion between reference and sample arm (Kottig et al. 2012). While dispersion between both interferometer arms, broadening the interference signal and by this decreasing the axial resolution, can be numerically compensated in FD-OCT, this is more complicated in TD-OCT (Wojtkowski et al. 2004).

For systems with high lateral resolution, TD-OCT offers the advantage that the focus position can be shifted synchronously to the length of the reference arm allowing high lateral resolution over a large depth range (Schmitt et al. 1997).

TD-OCT and FD-OCT can be parallelized in one or two dimensions by using additional detector dimensions. Especially in TD-OCT, using camera-based systems allows sampling of complete frames which is then called full-field OCT (FF-OCT) providing en face images sequentially (Dubois et al. 2002). The disadvantage in FD-OCT is that due to the longer measurement time for the extra dimensions(s), the image will be more compromised by movement artifacts (Yun et al. 2004). Moreover, all parallel systems are more sensitive to artifacts due to multiple scattering because of the loss of the confocal advantage (Garini et al. 2006).

Besides central wavelength, resolution, speed, and measurement range, two more parameters are used to characterize OCT systems. The first is the sensitivity specifying the minimal reflectivity of a plain surface that results in a signal equivalent to the noise level. Because of the interference principle, very low levels can be detected and typical values of the minimal reflectivity are in the range of $10^{-10}$ corresponding to a sensitivity of $100 \mathrm{~dB}$. Sometimes this parameter is called the signal-to-noise ratio (SNR) of the system as well. A second parameter is the dynamic range of the system which is the largest signal that does not saturate the detector or the electronics. Especially in FD-OCT, clipping of the signal, often caused by Fresnel reflexes, leads to strong artifacts and should be avoided. Due to the large dynamic range of OCT signals, mostly the logarithmic intensity data are visualized on a gray or false color map.

While in spectrometer-based FD-OCT systems, the limited resolution of spectrometers leads to a sensitivity roll off at the end of the measurement range of 10-20 dB (Bajraszewski et al. 2008), a similar degradation in swept source OCT systems is often caused by the limited frequency range of the detector and the coherence length of the sweeping light source. Additionally, the confocal principle leads to a signal drop outside of the focus that depends strongly on the used numerical aperture of the probe beam. Typical numerical apertures used in FD-OCT are therefore in the range of 0.05 , yielding transversal resolutions of typically 10 wavelengths.

While alterations by transversal motion in images from SD-OCT and OFDI systems are similar, both are affected quite differently by axial sample motion (Yun et al. 2004). Axial motion will lead to fringe washout in the case of SD-OCT, while it leads to false positions in the case of OFDI. Oblique motion can in general not be treated as the sum of both effects (Walther et al. 2008).

\subsubsection{Extended Functionalities}

\subsubsection{Spectroscopic OCT}

Spectroscopic OCT (S-OCT) is an extension of the intensity-based backscattering images in standard OCT. Since OCT images are recorded with broadband light, the spectral distribution of the light will be affected by absorption and scattering in the tissue. In other words, if 
characteristic absorbers or scatterers are present in the tissue, they will imprint characteristic features into the spectra of the backscattered light. These characteristic features may be analyzed to add spectroscopic information to the OCT images by an additional color map. This may enable a kind of "optical histology" including digital staining based on absorbing chromophores or characteristic sizes of scatterers (Kartakoullis et al. 2010; Robles and Wax 2010; $\mathrm{Xu}$ et al. 2005) inside the tissue. For example, in the near infrared, hemoglobin is a relevant substance for functional imaging with OCT (Faber et al. 2005; Lu et al. 2008; Robles and Wax 2010) because its state of oxygenation influences its spectral properties. Other chromophores in the tissue with less pronounced spectral features in the optical window include melanin, bilirubin, and water (Anderson and Parrish 1981). The spectra are also sensitive to scatterers which are in the same order as the wavelength of the light. This may be used to analyze tissue structures below the resolution of the OCT system (Robles and Wax 2010; Yi et al. 2013). In addition to the existing tissue ingredients, contrast agents for S-OCT, for example, dyes (Xu et al. 2004) or nanoparticles (Lee et al. 2003; Oldenburg et al. 2009), may be used.

In principle, there are hardware-based and software-based S-OCT concepts. The hardwarebased concepts use multi-band systems to obtain spectrally resolved OCT images in a differential manner (Cimalla et al. 2009; Storen et al. 2006). Software-based approaches use time-frequency distributions that extract the spectroscopic information from the raw OCT data, which can be recorded with standard OCT systems. In a first attempt, Morgner et al. performed spectroscopic analysis using a software-based approach on the basis of TD-OCT data (Morgner et al. 2000), while Leitgeb et al. used a FD-OCT system (Leitgeb et al. 2000). In practice, S-OCT still faces serious challenges. First, spectral features are often weak and superimposed by noise-like speckle and wavelength-dependent optical aberrations of the OCT system (Faber et al. 2005; Kasseck et al. 2010). Moreover, using time-frequency distributions, there is a tradeoff between spectral resolution and spatial resolution that has to be considered (Robles et al. 2009). But in spite of these difficulties, recent work has demonstrated that spectroscopic analysis may add significant contrast to the OCT images (Fleming et al. 2013; Jaedicke et al. 2013; Robles et al. 2011; Yi et al. 2014).

\subsubsection{Polarization-Resolved OCT}

In order to get more information or another contrast in OCT images, polarization-sensitive OCT (PS OCT) was introduced. Aligned structures like muscles or fibers show birefringence and thereby influence the polarization of the beam allowing depth-resolved measurement of the distribution and orientation of these structures. Birefringence of the sample might influence images even in normal OCT systems, resulting in horizontal stripes, as the polarization of sample and reference light from some depth will not be aligned, leading to a loss of signal (Cimalla et al. 2009). Most PS OCT systems detect at least two orthogonal linear polarization states of the reflected beam. In order to get similar intensities in both polarization states of the reference beam, a quarter wave plate is inserted into the reference arm. Moreover, a second quarter wave plate in the sample beam ensures circular polarization on the sample. Size and orientation of the birefringence can be calculated from amplitudes and phases of both interference signals (de Boer et al. 1997; Hee et al. 1992; Hitzenberger et al. 2001). While controlling the polarization is relatively easy in the case of bulk optics, the higher flexibility of fiber-coupled scanner heads demands for more complicated solutions (Al-Qaisi and Akkin 2010; Götzinger et al. 2009; Yamanari et al. 2008). One of the most important applications of PS OCT is the visualization of the retina. The retinal pigment epithelium (RPE) has the interesting property that it depolarizes the scattered light. Therefore, the homogeneity of polarization, called the degree of polarization uniformity (DOPU), is often shown as an additional contrast to differentiate this layer. PS OCT is also used to differentiate carcinoma and to distinguish between normal and burned tissue (Park et al. 2001). 


\subsubsection{Doppler-OCT}

The visualization and quantification of flowing blood are one of the main applications of Doppler-OCT (DOCT). DOCT was first introduced in TD-OCT by detecting the frequency shift of the interference signal caused by axially moving scatterers (Xu et al. 2008). Much higher sensitivity to axial movement can be achieved by analyzing the phase of the interference signals in consecutive A-scans at the same or nearly the same position (Leitgeb et al. 2003b). This method, called phase resolved Doppler-OCT, is today used in TD-OCT and FD-OCT. A high spatial oversampling yielding a great overlap of the two beam profiles is beneficial for a good sensitivity of DOCT (Walther and Koch 2009). Other methods using phase modulators in the reference arm (Bachmann et al. 2007) or changing the length of the reference arm to highlight moving structures (Wang et al. 2007b) have shown remarkable results. As phase data are often very noisy, different ways of averaging have been suggested. Most commonly, the phase $\varphi$ is calculated by calculating the phase of the sum of products of consecutive A-scans:

$$
\varphi=\arg \left[\sum_{j}{ }_{j+1}(z) \cdot \Gamma_{j} *(z)\right]
$$

$\Gamma_{j}(z)$ is the complex result of the FFT of the A-scan $j$ at depth $z$ and "*" denotes the complex conjugate. In the case of low SNR, analyzing a set of A-scans using a 2D Fourier transformation, called joint spectral and time domain OCT (STdOCT), may be beneficial (Szkulmowski et al. 2008). More sophisticated strategies for averaging Doppler data can be found in Chan et al. (2014) and Walther et al. (2014).

Doppler information is mostly visualized by a false color map, where the intensity is given by the logarithmic intensity of the signal and the color from blue over white to red indicates the phase in the interval of $\pm \pi$, with white indicating no axial motion. In the case of smooth velocity distribution, phase shifts much above $\pm \pi$ can be analyzed by unwrapping techniques, but in SD-OCT artifacts caused by the detector integration time may cause huge discrepancies at higher flow velocities depending on the angle of the flow (Walther et al. 2010).

An example of a phantom measurement, highlighting potentials and problems of the flow through different capillaries, is shown in Fig. 16.15. The different images show human blood or $1 \%$ intralipid solution, often used as a substitute for blood in Doppler measurements, through a rectangular and a circular capillary in opposite directions. The angle of both capillaries was the same. While the top row shows only the intensities, the bottom row is colored as a function of the phase shift or velocity. To reduce the influence of the speckle noise, $256 \mathrm{~B}$-scans are averaged. In spite of this averaging, the left images without flow still show the granularity of blood. The echo from intralipid is quite homogeneous as can be seen in the images on the right. Vice versa flowing blood shows echoes in the form of an hourglass, caused by the circular orientation of erythrocytes in flowing blood (Cimalla et al. 2011a). Especially at the backreflections from the capillaries in the case of blood, high phase shifts can be seen. This effect, called Doppler shadow (Bukowska et al. 2010), is attributed to multiple scattering (Kalkman et al. 2012) and is less pronounced in the case of intralipid.

Besides algorithms based on the phase shift, intensity-based algorithms evaluating the variance in repetitive scans have been used especially for angiography showing high contrast images of blood vessel structure in the hamster skin, for example (Liu et al. 2011). A comparison of different methods and their advantages and disadvantages is found in (Liu et al. 2012).

An application for Doppler imaging in small animals is the observation of blood flow in vessels of mice. Blood flow in these vessels is regulated by the contraction of the vessel muscles in the arteries. As the veins have no musculature, their diameter will only change passively by inner or outer pressure. While vasoconstriction may be caused by application of potassium, vessels will dilate after the application of sodium nitroprusside (SNP). A dysfunction of this regulation mechanism is associated with arteriosclerosis. Due to its advantageous position, imaging was performed at the arteria and vena saphena 

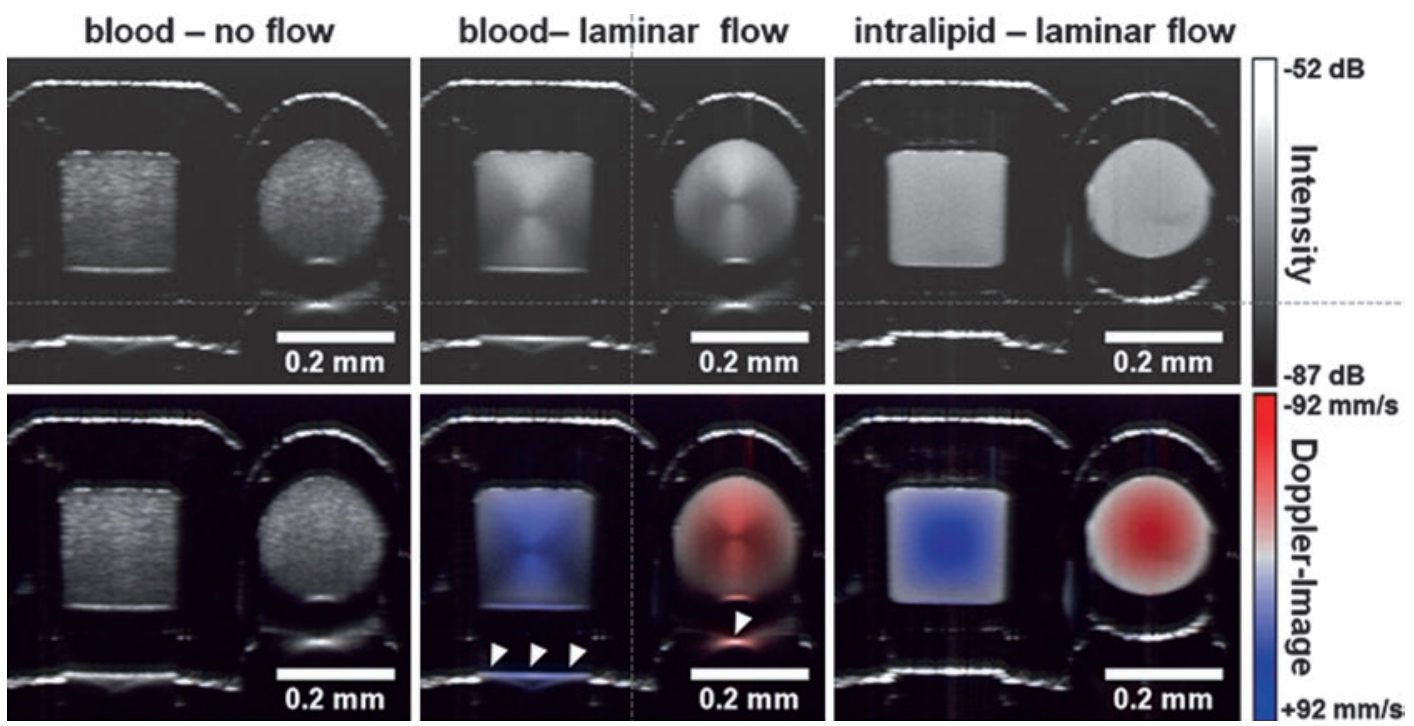

Fig. 16.15 Phantom measurement for Doppler imaging with OCT. The upper part on the left side shows the image of blood with no flow. Below additional Doppler processing shows no difference. The images in the middle show corresponding images for flowing blood. The square capillary and the circular capillary have opposite flow directions. Echoes behind the structure show the so-called

with a dual-band OCT system. The dual-band system nicely shows the higher phase changes at the smaller wavelength. Quasi M-scans are shown in the middle and bottom of Fig. 16.16, showing the oscillating blood flow in the artery and the almost constant blood flow in the vein. The low amplitude of the backscattered signal in the center of the vessels is attributed to the alignment of the erythrocytes as stated above.

\subsubsection{Contrast Enhancement by Nanoparticles: Photothermal OCT and Magnetomotive OCT}

Compared to other optical imaging techniques such as fluorescence microscopy, OCT is a rather unspecific modality that provides only general information on the tissue structure based on light scattering on cells and cellular components such as the nuclei and mitochondria. Hence, to improve visibility of specific tissues or cells, OCT contrast agents are required. Several attempts have been made to find a suitable contrast agent for OCT including dyes and plasmon-resonant nanoparti-
Doppler echo which is attributed to multiple reflections. The inhomogeneous structure is attributed to the alignment of erythrocytes in the flowing blood. The images on the right side are taken with intralipid showing less Doppler echo and no inhomogeneity. To reduce the speckle noise, the images were averaged over $50 \mathrm{~B}$-scans

cles, which have distinct absorption spectra and therefore can be detected by means of spectroscopic OCT (Cang et al. 2005; Oldenburg et al. 2009; Xu et al. 2004; Yang et al. 2004a). However, these absorption-based methods always suffer from an inherent concentration-path length tradeoff. In order to detect a sufficient absorbance, either a long propagation length through the absorber is required, which consequently degrades axial resolution, or a high concentration of absorbing agents is necessary, which is often not tolerated by the specimen.

However, since OCT is very sensitive to motion due to the coherent nature of the illuminating light, the most promising approaches photothermal and magnetomotive OCT - use dynamic contrast agents to enhance visualization of specific substances.

In photothermal OCT, gold nanoparticles inside the sample are remotely heated by illumination with intense laser light ( $>20 \mathrm{~mW})$. For that purpose, the emission wavelength of the laser is matched to the plasmon resonance absorption maximum of the particles. The illuminated 


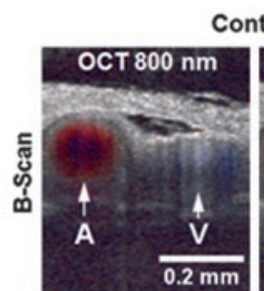

Control
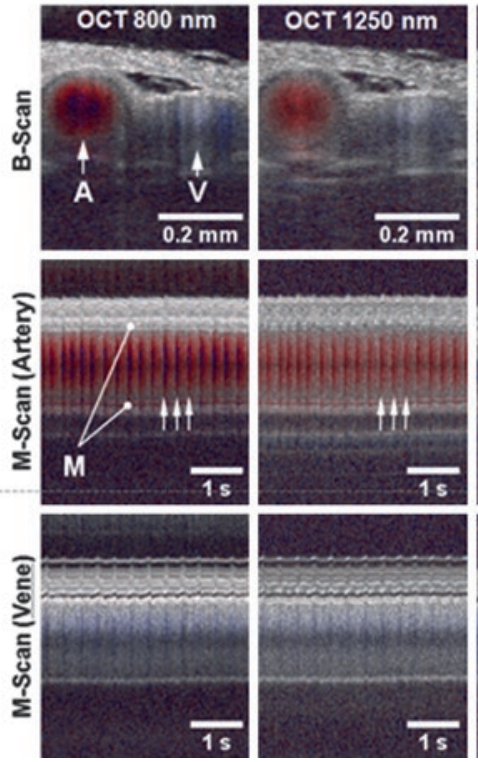

Vasoconstriction (Potassium)
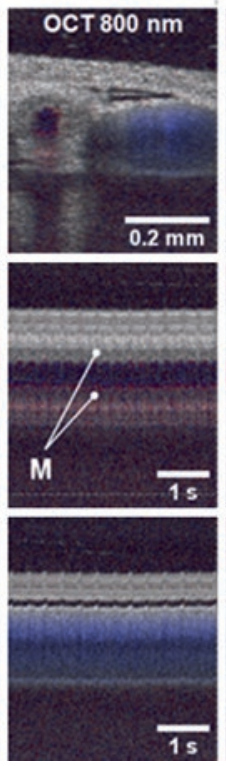
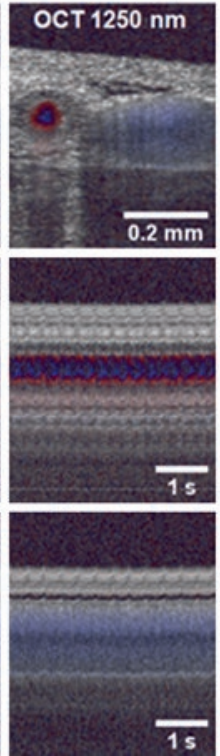

Vasodilatation (SNP)

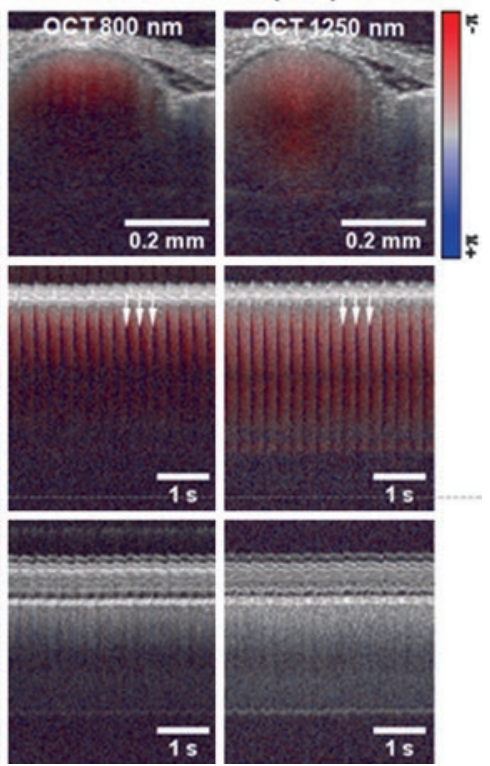

Fig. 16.16 Top row: single B-scans showing a cross section through the artery and vein in mice. Left side control, middle vasoconstriction after the application of potassium, right side vasodilatation caused by the application of SNP. As stated in the columns, images were taken at the same time at center wavelengths of $800 \mathrm{~nm}$ and $1250 \mathrm{~nm}$. Due to the shorter wavelength, the phase change is larger in the $800 \mathrm{~nm}$ band. Related to this, a phase wrap (phase change more than $\pm \pi$ ) is first seen in the $800 \mathrm{~nm}$ band.

particles also heat their immediate vicinity causing thermal expansion of the microenvironment. Hence, resulting sample motion in the submicron regime is then detected by OCT using phase information (Adler et al. 2008). Sufficient sensitivity is achieved by modulating the laser (turn on/turn off periodically) and searching for the corresponding frequency in the OCT phase signal. Photothermal heating can be performed at relatively high frequencies of $0.5-20 \mathrm{kHz}$ (Zhou et al. 2008), which enables fast image acquisition.

Therefore, among others, photothermal OCT was successfully applied for pharmacokinetic studies to noninvasively image nanoparticle accumulation in mouse mammary tumors (Tucker-Schwartz et al. 2014).

Furthermore, photothermal OCT is not necessarily bound to the presence of gold nanoparticles. Also, the absorption profile of endogenous chromophores such as hemoglobin can be used
Nevertheless, in the case of vasoconstriction, phase wrap is seen in both bands. A-scans selected in the center of the artery (middle) and vein (bottom), indicated by $A$ and $V$ in the upper left part, from sequential B-scans, forming a quasi M-scan, highlight the highly pulsating blood flow (see arrows) in the artery and the slightly modulated blood flow in the vein. The musculature of the artery $(M)$ can be nicely seen, especially in the case of vasoconstriction

for imaging (Yim et al. 2014). Since the absorption spectra of oxy- and deoxyhemoglobin differ, photothermal OCT can be applied to assess the blood oxygenation level both in vitro (Kuranov et al. 2011a) and in vivo, e.g., in the murine brain cortex (Kuranov et al. 2011b).

However, attention must be paid to keep sample temperature below $44{ }^{\circ} \mathrm{C}$ to avoid thermal damage of biological tissue. Furthermore, photothermal OCT is not suited for imaging of photosensitive tissue such as the retina due to the use of intense light.

In magnetomotive OCT, magnetic micro- or nanoparticles are used as dynamic contrast agents, which are exited into motion by an external magnetic field. Contrast enhancement is then achieved by quantifying the displacement of the elastic tissue next to the particles using the amplitude or phase information of the OCT signal (Oldenburg et al. 2005a, 2008). 
In this context, it was shown that magnetomotive OCT is a suitable method for contrastenhanced imaging of single magnetically labeled cells in vitro (Oldenburg et al. 2005b). Proof-ofprinciple in vivo magnetomotive imaging was demonstrated in African frog (Xenopus laevis) tadpole (Oldenburg et al. 2005a) as well as in the mouse eye (Wang et al. 2010b). Furthermore, similar to photothermal OCT, magnetomotive OCT was also successfully applied to image the accumulation of targeted magnetic nanoparticles in rat mammary tumors (John et al. 2010).

Typically, superparamagnetic iron oxide particles are used for labeling of biological samples. Compared to ferromagnetic materials, these particles exhibit lower forces in a magnetic field due to their smaller magnetization capability. However, iron oxide is much better suited in terms of biocompatibility since pure iron in higher concentrations is cytotoxic.

In contrast to photothermal OCT, modulation of the magnetic field in magnetomotive OCT is restricted to relatively low frequencies of 0,1-1 kHz (Kim et al. 2013) since fast switching of sufficiently high magnetic fields is technically challenging. Furthermore, magnetomotive imaging is limited to small working distances because magnetic field strength rapidly degrades with distance from solenoid.

\subsubsection{OCT for Small Animal Imaging}

\subsubsection{Retina Imaging}

The retina was one of the first samples investigated with OCT, since it has the unique advantage to be visualized through the transparent ocular media. In this context, OCT provides in vivo cross-sectional visualization of the retinal microstructure that cannot be obtained by any other noninvasive imaging modality (Drexler and Fujimoto 2008). Therefore, the clinical application of OCT is mostly developed in ophthalmology for the diagnosis, staging, and monitoring of ocular diseases primarily macular pathologies such as age-related macular degeneration (AMD), central serous retinopathy, macular holes and edema, Stargardt disease, and retinitis pigmentosa but also optic neuropathies such as glaucoma. Today, several commercial ophthalmic OCT devices are available, which use high-speed spectral domain detection. All instruments have comparable performance with an axial resolution of 4-6 $\mu \mathrm{m}$ operating in the $800 \mathrm{~nm}$ wavelength range and imaging speeds of 25,000-40,000 axial scans per second (Wolf-Schnurrbusch et al. 2009), which enables real-time two-dimensional imaging of tissue cross sections.

As an alternative or extension to the conventional ophthalmic imaging at $800 \mathrm{~nm}$, research OCT systems operating at $1050 \mathrm{~nm}$ have also been demonstrated. Due to less absorption of the retinal pigment epithelium and reduced tissue scattering on the one hand and still sufficiently low light absorption by means of the aqueous ocular media in front of the retina on the other hand, this spectral range allows depth-enhanced visualization of the choroid (Povazay et al. 2003; Unterhuber et al. 2005) and improved visibility of the retina in patients with opaque ocular media, i.e., cataract (Povazay et al. 2007).

As stated above, one particular feature of OCT is that the axial resolution is decoupled from the transverse or lateral resolution. The axial resolution is mainly given by the coherence length of the applied light source, while the lateral resolution is defined by the numerical aperture of the focusing optics, i.e., the beam diameter and the focal length, like in conventional microscopy. In ophthalmic imaging, the eye's cornea and lens are used as the imaging objective. Therefore, the lateral resolution is limited by the pupil size and the focal length of the eye and, in practice, even further reduced by ocular aberrations. In order to overcome this practical limitation, adaptive optics - well known in the field of astronomy - is applied in research OCT systems to correct ocular aberrations. These instruments measure the wavefront distortion caused by the ocular media and correct it by means of deformable mirrors or liquid crystal phase modulators. It was shown that adaptive optics can significantly improve lateral resolution to $2-3 \mu \mathrm{m}$ compared to $15-20 \mu \mathrm{m}$ in conventional ophthalmic OCT (Povazay et al. 2009). Furthermore, this technique increases 
imaging sensitivity especially in the range of the photoreceptor layer and reveals single structures located at the inner/outer photoreceptor segment junction and the posterior tips of the photoreceptor outer segments, which are assumed to represent individual cone cells in humans (Fernández et al. 2008; Zhang et al. 2006) as well as in animal models such as mice (Jian et al. 2013). The combination of high axial resolution OCT with aberration-correcting adaptive optics is therefore often referred to as cellular imaging of the living retina.

For the understanding of the mechanisms underlying retinal degeneration and trial of potential therapeutic strategies, experimental animal models play an important role. In this context, OCT also enables noninvasive monitoring of the retina in small animals like rats (Nagata et al. 2009), mice (Horio et al. 2001) and even zebrafish (Bailey et al. 2012; Weber et al. 2013). The last-mentioned one is of particular interest for researchers and vision scientists since the zebrafish has the ability to regenerate neuronal tissue including the retina - a phenomenon that is not observed in mammals. Although animal imaging is mostly performed in the $800 \mathrm{~nm}$ wavelength range (Huber et al. 2009; Kocaoglu et al. 2007; Ruggeri et al. 2007) as in humans, OCT of the rodent retina is also reported at $1060 \mathrm{~nm}$ (Hariri et al. 2009) and $1300 \mathrm{~nm}$ (Li et al. 2001). It was shown that there is a good correlation between OCT data and histology (Huber et al. 2009), thus enabling OCT to be a valuable tool to analyze and monitor microstructural changes in the retina for noninvasive time-course studies in individual animals.

An example for in vivo OCT imaging and monitoring of the mouse retina is depicted in Fig. 16.17.

In human retinal imaging, the sample beam is conventionally relay imaged onto the pupil by means of a lens pair and focused onto the fundus by the eye itself, which was also applied for retinal imaging in mice and rats (Kocaoglu et al.
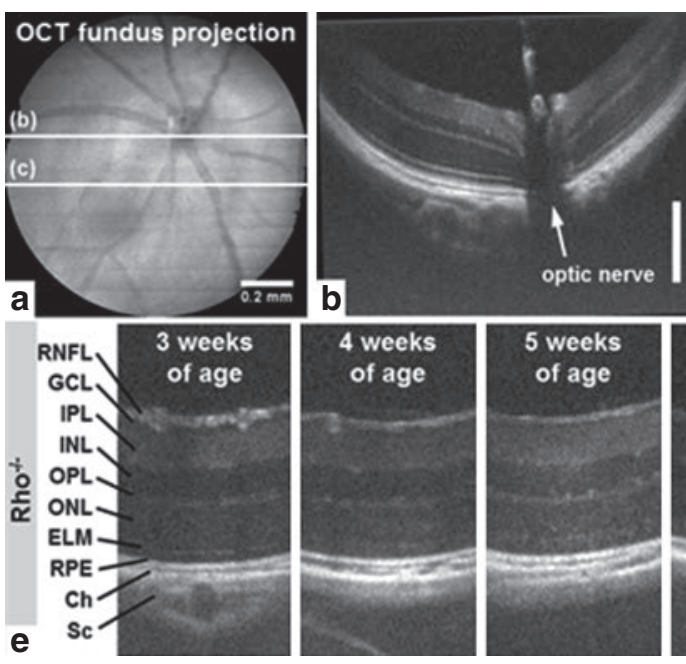

Fig. 16.17 High-resolution OCT imaging of the mouse retina in vivo. (a) OCT fundus projection and representative OCT cross sections $(\mathbf{b}, \mathbf{c})$ of the healthy mouse retina (vertical bars, $200 \mu \mathrm{m}$ ). (d) Detailed view of the retinal microstructure (abbreviations: RNFL retinal nerve fiber layer, $G C L$ ganglion cell layer, $I P L$ inner plexiform layer, $I N L$ inner nuclear layer, $O P L$ outer plexiform layer, $O N L$ outer nuclear layer, ELM external limiting membrane, IS/ $O S$ inner/outer photoreceptor segment junction, RPE retinal pigment epithelium, $C h$ choroid, $S c$ sclera). (e) Longitudinal OCT monitoring of genetic retinal degener-
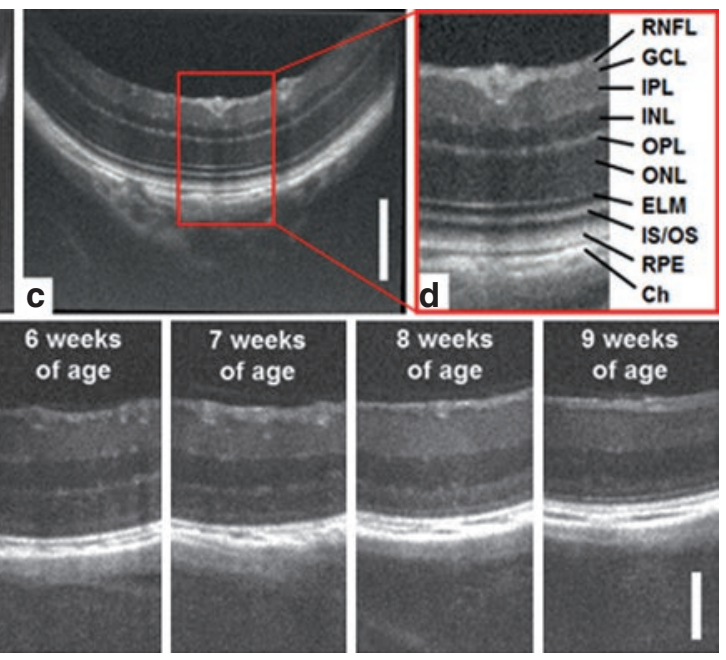

ation in one individual rhodopsin-deficient $\left(\mathrm{Rho}^{-/-}\right)$mouse at different ages (vertical bar, $100 \mu \mathrm{m}$ ). The $\mathrm{Rho}^{-1-}$ degeneration as a model for recessive inherited retinitis pigmentosa is characterized by a progressive loss of the outer nuclear layer (ONL), which represents the nuclei of the photoreceptors. At an age of 9 weeks, the ONL has almost completely vanished, so that only a minor fraction of photoreceptors is left at this stage. All images were acquired with an experimental high-resolution dual-band OCT system (Cimalla et al. 2009, 2011b) 
2007; Ruggeri et al. 2007). However, it is reasoned by others that this method is not optimal for small animals since the small size and short focal length of the eye imply a high numerical aperture objective lens design in order to cover a large measurement area on the retina. In addition, the short focal length of the eye increases aberration, which degrades the OCT signal and reduces image quality. Alternatively, a contact lens made from a microscope coverslip and hydroxypropyl methylcellulose solution is used, which effectively removes the refractive power of the aircornea boundary and allows the sample beam to be focused directly onto the retina. Due to the corresponding enlargement of the eye's focal length, lower numerical aperture lens designs can be applied to cover a sufficient measurement area on the retina. Furthermore, this technique reduces corneal aberrations and prevents the eye from dehydration (Kim et al. 2008; Srinivasan et al. 2006), which would otherwise lead to corneal opacity.

Due to their small sizes, it is possible to image the entire eyes of mice and zebrafish from the cornea to the retina in one single OCT scan. This technique allows noninvasive quantitative assessment of biometric parameters such as intraocular distances and interface curvatures of ocular media, which is of interest in eye development research (Wang et al. 2010c; Zhou et al. 2008) and eye modeling for improved design of optical instruments (Gaertner et al. 2014).

Recent developments in the experimental and commercial sector tend toward multimodal imaging of the small animal retina using OCT in combination with other established methods such as scanning laser ophthalmoscopy (SLO), fluorescence angiography (FA), and fundus autofluorescence (FAF), which deliver additional information about fundus morphology, blood flow, and metabolism (Fischer et al. 2010; Huber et al. 2009, 2010; Seeliger et al. 2005).

An example for multimodal in vivo imaging of the mouse retina using OCT and fluorescence angiography is shown in Fig. 16.18.

In addition to the morphological and molecular contrast acquired by optical imaging techniques, functional information about photoreceptor and interneuron activity can be obtained by means of electroretinography (ERG). In this context, simultaneous OCT and ERG for parallel monitoring of the retinal structure and function in experimental animal models such as rats (Moayed et al. 2010) allows advanced analysis of retinal degeneration processes in vivo.

Furthermore, attempts have been made to retrieve functional information from the OCT signal itself. To that, local changes in the retina's optical properties following to light stimuli are detected by means of OCT. These intrinsic optical signals, which could by now be observed in the human retina (Schmoll et al. 2010) as well as in chicken (Moayed et al. 2011), were found to be restricted to the stimulated fundus region and to show some correlation with ERG recordings.

\subsubsection{Lung Imaging}

Due to a lack of imaging technologies having sufficient resolution for resolving single alveoli and delivering three-dimensional data at high frame rates, there is a high interest in using OCT for imaging airway wall and alveolar structures in animal studies. Lung function in healthy and diseased lungs is of high interest for several reasons. Some interest is related to the transport of inhaled particles for drug delivery and risk due to smoking (Yin et al. 2009). Further airway remodeling in connection with asthma may reduce the airway distensibility. Moreover, interest in visualization of alveoli and alveolar structures is related to the high morbidity of acute respiratory distress syndrome (ARDS) and ventilatorinduced lung injury (VILI), which in spite of numerous efforts declines only slowly. A better understanding of processes during artificial ventilation might help in improving ventilation strategies.

Several strategies have been applied to image airway and alveolar structures with OCT. External scanning can be applied to isolated lungs visualizing the alveolar structure close to the pleura (Meissner et al. 2009b; Mertens et al. 2009). With some effort, this method can also be applied to anesthetized animals by opening the chest or by preparing a thoracic window in vivo (Meissner et al. 2010a). This method has been applied to 

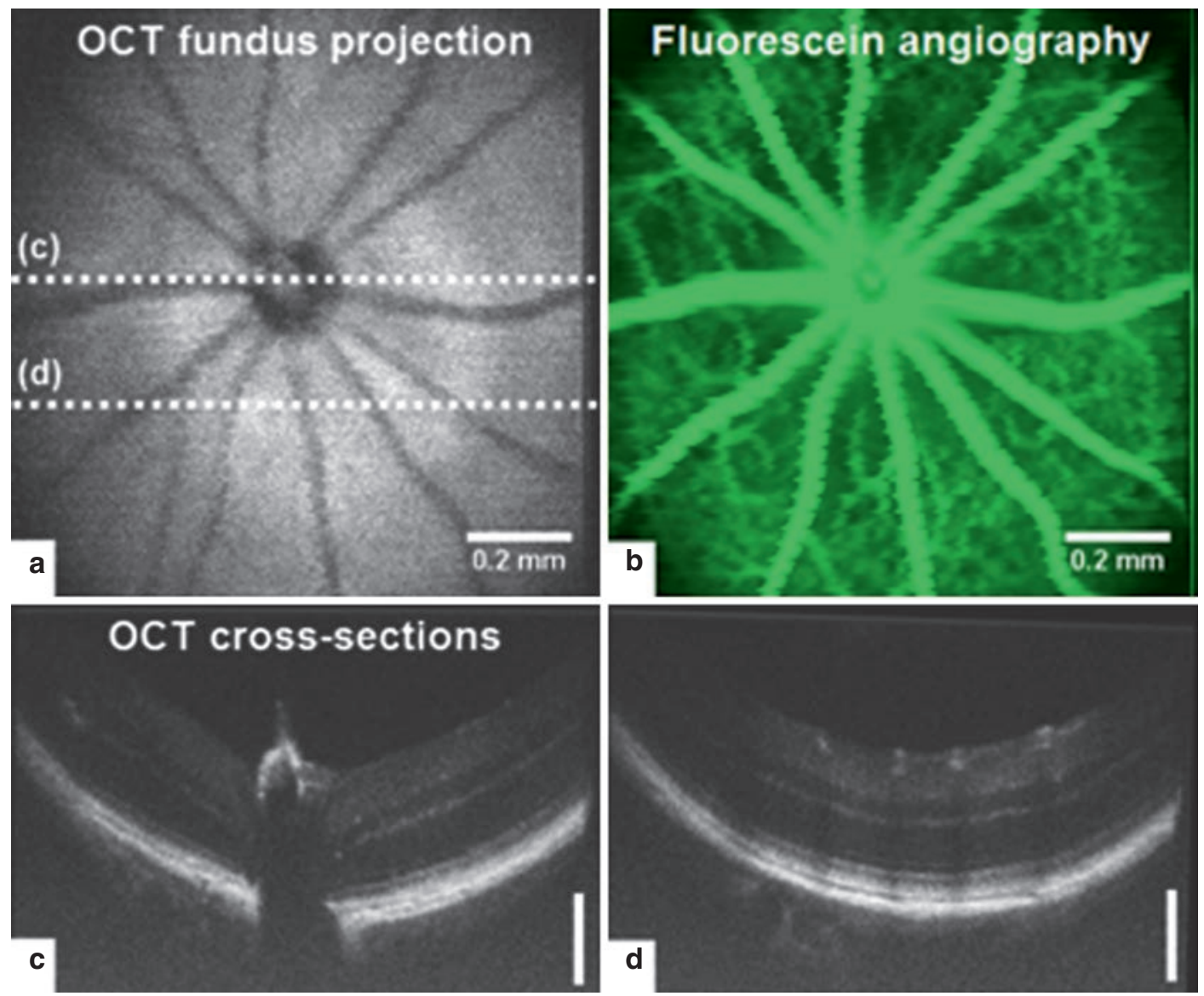

Fig. 16.18 Multimodal in vivo imaging of the mouse retina. (a) OCT fundus projection and (b) simultaneously acquired fluorescence angiography image of the healthy mouse retina. (c, d) Representative OCT cross sections (vertical bars, $200 \mu \mathrm{m}$ ). For fluorescence angiography, the

approved in vivo dye fluorescein was used, which has its emission maximum in the green visible spectral range. All images were acquired with an experimental hybrid imaging system slightly modified to (Cimalla et al. 2012)

mouse and rabbit models to study alveolar sizes and the change during ventilation in healthy and injured lungs. While in the early systems, gated imaging was used to study the dynamic shape during the ventilation cycle (Meissner et al. 2010b), modern high-speed systems capture complete alveoli with some tens of frames per second, sufficient for breathing rates in the order of one per second (Kirsten et al. 2013). In larger animals the movement of the lung prevents following single alveoli during the complete ventilation cycle. Therefore, lightweight MEMS-based probes were developed resting on the lung and following the alveolar structures (Namati et al. 2013).

To access internal regions of the lung, endoscopic OCT probes have been used. Typically, such probes use radial scanning by rotating the entire probe or by using micromechanical motors placed in the probe (Yang et al. 2004b). While the rotation provides circumferential $2 \mathrm{D}$ images, the fiber may be retracted while accumulating subsequent radial images to provide three-dimensional information.

A third strategy uses miniaturized OCT needle probes. The needle probe can be inserted through the tissue into the region of interest that may be located several centimeters below the surface. Scanning is again performed by rotating the needle or the focusing optics inside the needle. 
Although the size of needle probes has been reduced to a diameter of about $310 \mu \mathrm{m}$, the insertion of the needle will cause some trauma (Lorenser et al. 2011). As the needle can hardly be translated forth and back, the same tissue cannot be imaged during different states of the ventilation cycle. For repetitive scans, probes consisting of an inner part that translates rapidly back and forth multiple times per second were developed allowing two-dimensional image acquisition (McLaughlin et al. 2012).

Imaging alveolar structures is hampered by the repetitive refractive index change from air to liquid, limiting the useful depth to only one or two layers of alveoli. Therefore, several attempts have been made to fill the lung with an index matching fluid. For ex vivo imaging, the lung can be filled completely with saline water (Lorenser et al. 2011) or ethanol (Meissner et al. 2009a) increasing the useful imaging depth to 5 or more alveoli in depth. While these approaches are not suited for in vivo imaging, a small amount of fluid around the probe can enhance imaging without scarifying the animal (Quirk et al. 2014).
Moreover, liquid ventilation using perfluorocarbons having a high transport capacity for oxygen and carbon dioxide can be used for ventilation allowing enhanced OCT imaging in vivo (Schnabel et al. 2013) (see Fig. 16.19).

\subsubsection{Discussion and Conclusion}

Optical coherence tomography is a contactless optical imaging concept enabling threedimensional tomographic imaging with micrometer resolution. However, due to the strong scattering of light in biological tissue, the penetration depth and thus the image depth are limited to a few millimeters in the best case, when light in the optical window between 600 and $1300 \mathrm{~nm}$ is used. The imaging speed of state-of-the-art OCT systems is high enough to enable real-time video imaging.

Primarily, the contrast in OCT images is generated by refractive index variations at interfaces between different tissues that lead to backscattered light. Additional contrast mechanisms rely

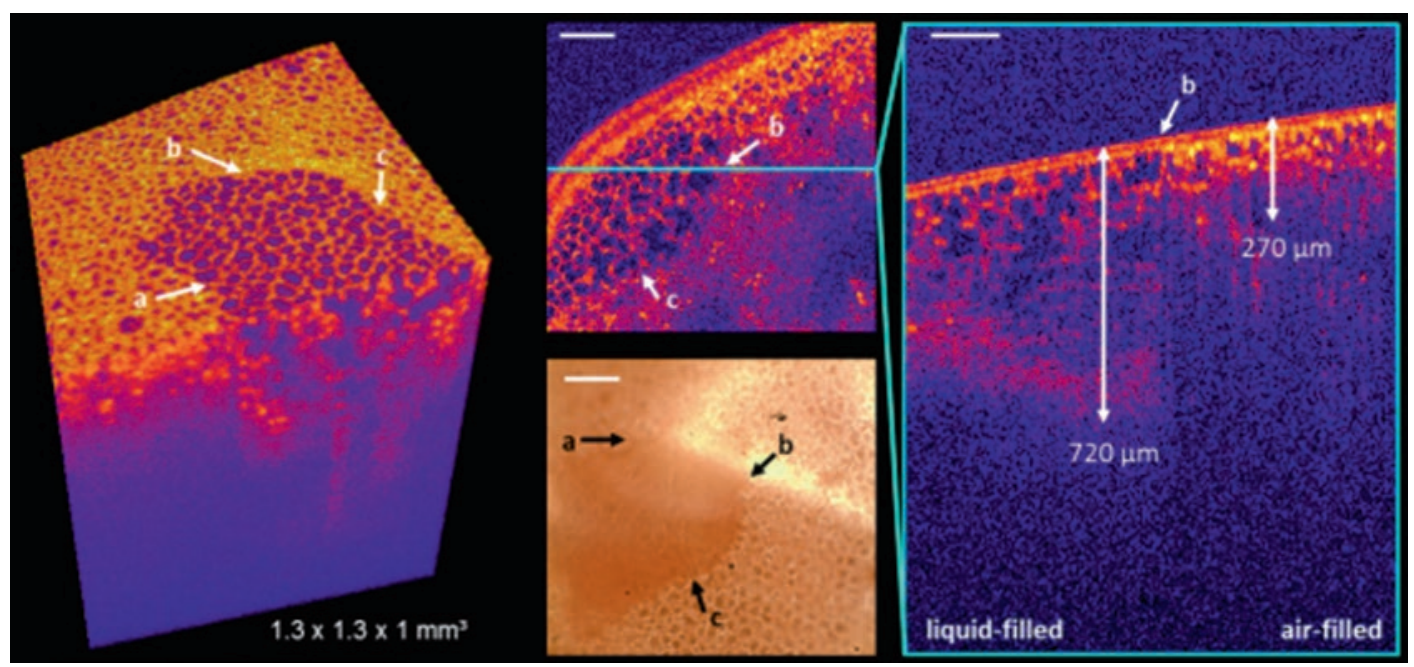

Fig. 16.19 Transition from an air-filled to a liquid-filled region in OCT and intravital microscopy (IVM). These pictures show the influence of liquid filling for OCT and IVM lung imaging after 18 min of total liquid ventilation. While image quality for OCT is considerably increased in the liquid-filled area, the contrast for IVM is decreased due to the refractive index matching. The depth of tissue visualization is nearly tripled in the liquid-filled area due to the reduction of scattering loss. The arrows $a, b$, and $c$ in the 3D OCT image (left) were used for a better understanding of the OCT and IVM en face view (middle row) and the OCT cross section (right). For the 3D image, the pleura was hidden to get a look on the first layer of subpleural alveoli. Scale bar is $200 \mu \mathrm{m}$ (From Schnabel et al. (2013) with permission) 
on spectroscopic modifications of the backscattered light by spectrally dependent absorption or scattering or on variations of the polarization due to birefringence in aligned structures like fibers or muscles. Contrast enhancement by adding contrast agents like nanoparticles has also been demonstrated.

In addition to stationary images of the tissue structure, so-called Doppler-OCT also enables visualization of movements in the tissue, for example, the blood flow.

In terms of small animal imaging, OCT has been successfully used for retina imaging. The transparence of the ocular media enables noninvasive imaging of the retina not only for humans but also for rats, mice, and zebrafish. This imaging is not restricted to stationary morphological images but also extends to functional imaging by detecting local changes of the retina's optical properties due to light stimuli.

Imaging of vessels has been successfully demonstrated. A particular application is the investigation of traumatic brain injuries or strokes in rat models (Osiac et al. 2014). Also, cardiovascular defects in mutant mouse animal models have been analyzed by OCT. Even the development of whole mouse embryos can be monitored in vivo with OCT (Larina et al. 2011). In more recent studies, OCT has been successfully applied to monitor drug-induced changes of the tissue in mouse models (Bell et al. 2013). These recent developments demonstrate that OCT has become a powerful tool for small animal imaging and that its potential for this application is by far not completely explored.

\subsection{Intravital Imaging in Small Animals}

Timo Rademakers and Marc A.M.J. van Zandvoort

Over the last decades, molecular and cell biology has greatly advanced our knowledge of dynamic processes at a molecular level, especially by the use of (static) in vitro techniques. However, to gain an understanding of the in vivo implications hereof, a need for translation toward in vivo experiments developed, in which dynamic events could be studied in real time and in their specific microenvironment. Intravital microscopy (IVM) is one of the techniques which allow such studies. While the technique is (mostly) invasive, it is able to (depending on the system) study dynamic processes at a resolution of several micrometers and therefore has become a widely used technique in many fields of biomedical research.

The idea of studying (sub)cellular processes in small animals in vivo is not new. Already in the nineteenth century, the first rudimental intravital studies have been described, involving transillumination microscopy of leukocyte behavior in the mesentery of a frog (Julius cohnheim 1839). From these initial intravital studies using transillumination, the field has developed greatly with aid of advances in microscopy, allowing at current detailed multicolor study of dynamic processes using the newest fluorescent techniques.

The purpose of this chapter is to give an overview of IVM techniques and applications, in which both classical intravital imaging and state-of-the-art multiphoton intravital imaging will be discussed, including examples from different fields of research.

\subsubsection{Classical Intravital Imaging}

Classical intravital imaging uses regular widefield microscopy systems, either using transillumination or fluorescence. These classical systems have been in use for several decades and are still being used to date. Classical IVM has mostly been applied in inflammation research (i.e., leukocyte adhesion and transmigration), but also in studies involving hemodynamics (Kiyooka et al. 2005) or thrombosis (Furie and Furie 2006). Classical IVM however has some intrinsic limitations, as will be discussed below.

\subsubsection{Brightfield Imaging/ Transillumination}

The first IVM studies were performed using brightfield illumination or transillumination in transparent tissues, eventually in combination 
with the use of differential interference contrast (DIC) or phase contrast to enhance visualization of various structures within the tissue. The greatest advantage of this type of IVM is its relative ease of use, such that it can be performed on many regular light microscopes equipped with a high-speed detection system. Moreover, the lateral resolution (i.e., X-/Y-resolution) is generally $\operatorname{good}( \pm 500 \mathrm{~nm})$. One of the most obvious disadvantages, however, is the need for transparency in the tissue under study, which in most small laboratory animals is very limited. Also, in thicker tissues problems with image blurriness arise, caused by out-of-focus contributions. This significantly reduces image contrast and resolution. In practice, only few tissues like the cremaster muscle in male animals (Thorlacius et al. 1997) or the mesentery are transparent enough to allow transillumination. In these tissues, this type of IVM has been used to study, e.g., leukocyte adhesion to, rolling along, and transmigration through microvasculature. Figure 16.20 shows a typical image of a capillary in the cremaster muscle of a mouse, in which the capillary wall as well as the blood cells may be appreciated after applying a DIC filter.

\subsubsection{Widefield Fluorescence and Confocal Microscopy}

An approach which is similar to transillumination IVM is the use of widefield fluorescence microscopy. This technique offers a higher con- trast at comparable resolution, since it only detects specifically labeled fluorescent structures or cells, while the background - with exception of autofluorescent molecules - remains black. In addition, since fluorescent markers are available in different colors, it is possible to study differentially labeled tissue/cells at the same time using dual or triple filters. Moreover, higher contrast allows imaging of a multitude of tissues and organs, e.g., the liver (Kubes et al. 2002), noninvasive imaging of the capillaries in the murine ear (Lammermann et al. 2013), or even larger vessels such as the carotid artery (Fig. 16.21) (Massberg et al. 2003). However, out-of-focus blurriness problems still arise, which in practice still limits penetration depth to several micrometers in most tissues.

To introduce axial resolution, confocal microscopy can be used. In confocal microscopy, a laser system is used for excitation of fluorophores at their specific wavelength. Out-of-focus emitted light is filtered out by the use of pinholes, which reduces blurring of images up to $50-100 \mu \mathrm{m}$ of penetration depth depending on the type of tissue. Also, $\mathrm{XY}$ resolution is improved with a factor 2 . It is important to note, however, that when using line scanning systems, the acquisition speed is lower than in widefield systems, due to the stepwise buildup of the image, instead of direct acquisition of the entire image. To again gain imaging speed, systems can be fitted with a line scanning, resonant scanning, or
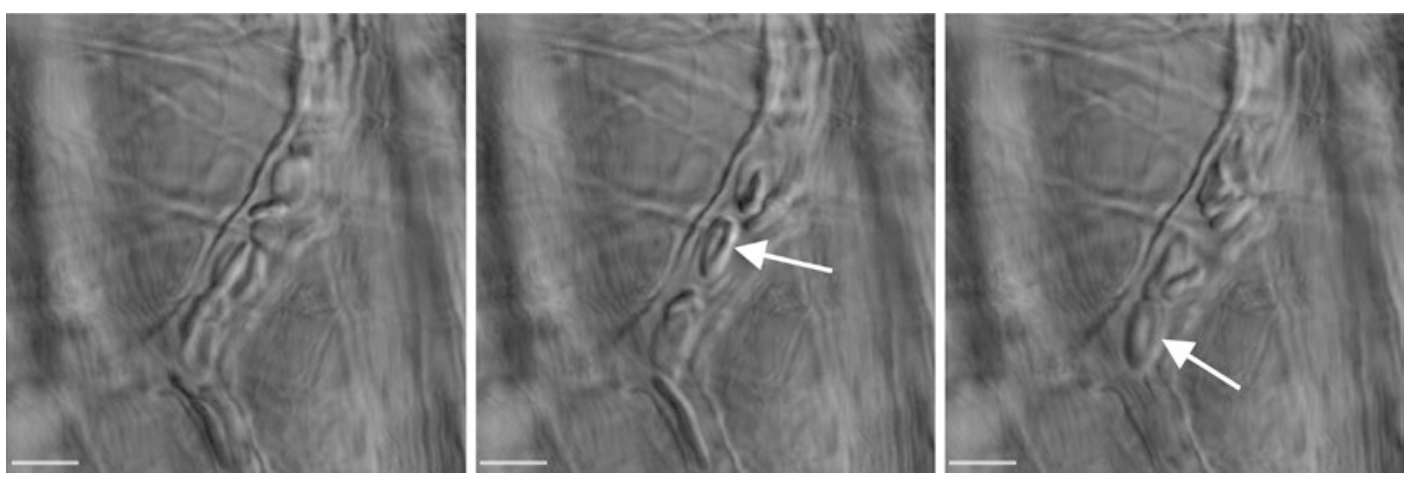

Fig. 16.20 IVM time series using transillumination microscopy. Thin tissue samples can be imaged by using DIC or phase contrast. Here, an erythrocyte (white arrow) can be seen passing through a capillary in the cremaster muscle. Upon an inflammatory stimulus, transmigration of leukocytes may also be determined 


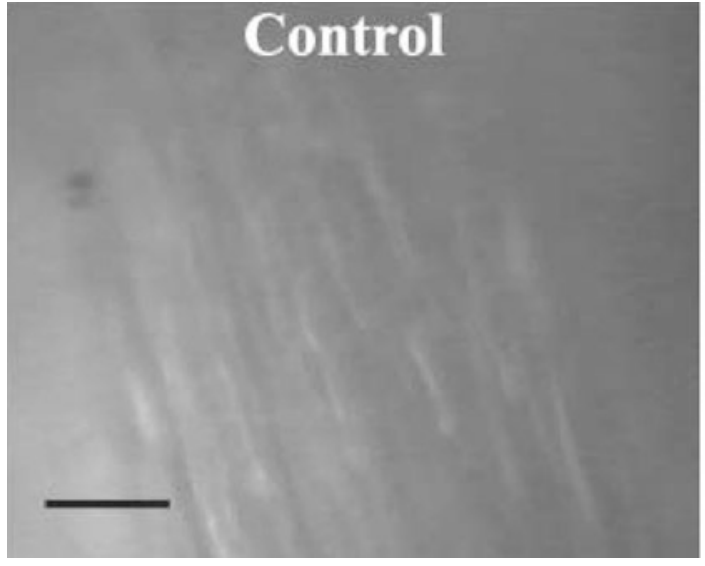

Fig. 16.21 IVM recording by widefield fluorescence microscopy of blood platelet (white, arrow) adhesion and aggregation in the common carotid artery of a mouse upon vascular injury. Widefield fluorescence IVM can be used

spinning disk system, which however causes some reduction in resolution and introduces noise. Alternatively, scanning multiple colors at the same time also enhances imaging speed, although not all systems are equipped for this task, as it requires specific fluorescent filters and/ or dual-camera systems. Nevertheless, depending on the system setup, high-speed multicolor IVM can be achieved.

The applications of these types of IVM are numerous, as long as there is the possibility to introduce a fluorescent label (either genetically or via other means) which is required for detection. Obviously, these types of IVM can be applied to the previously mentioned applications in brightfield IVM, i.e., imaging of the microvasculature in different tissues. Yet, since transillumination is no longer an issue, larger structures such as the macrovasculature can be studied in fluorescence-based IVM. Indeed, this technique has, e.g., frequently been used to assess leukocyte adhesion to the vessel wall of the carotid artery under inflammatory conditions or after thrombosis (Massberg et al. 2003), also harboring the potential to study differentially labeled cells at the same time, e.g., wild-type vs. knockout cells. The same holds true for confocal IVM in, e.g., the cremaster muscle, in which leukocyte transmigration can be studied in detail in the

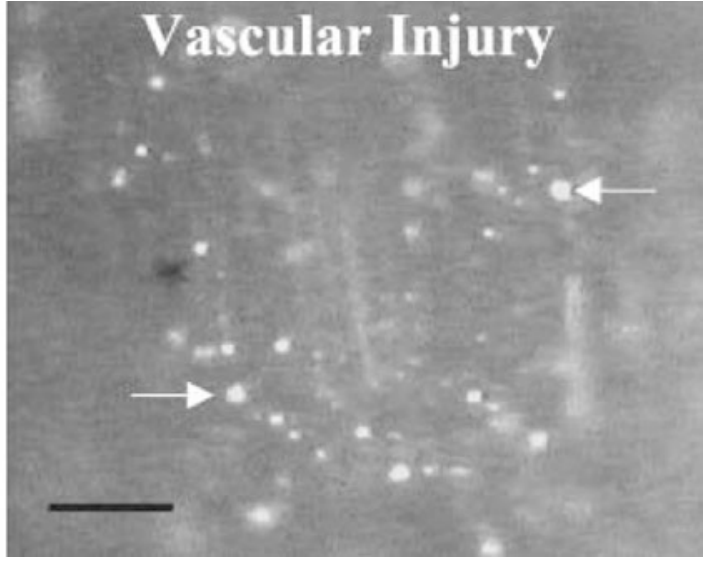

to assess increases in platelet adhesion and aggregation. Nevertheless, axial resolution in widefield fluorescence IVM is low (Figure is adapted from Massberg et al. (2003) and reprinted with permission)

microvasculature (Woodfin et al. 2011). Similarly in other fields like tumor biology, fluorescencebased IVM is often used in combination with dorsal skin chambers (Endrich et al. 1988; Menger et al. 2002).

\subsubsection{Intravital Multiphoton Imaging}

Even though classical intravital imaging is applicable in many cases, the intrinsic limitations with regard to axial resolution and penetration depth limit the use of these systems. New methods of intravital imaging, however, have become available over the past two decades. As a derivative of the confocal imaging systems, multiphoton microscopy was introduced early 1990s (Denk et al. 1990; Helmchen and Denk 2005). Multiphoton (MP) microscopy, mostly twophoton microscopy, uses multiple photons of higher wavelengths to excite fluorescent probes, in contrast to single-photon excitation in regular fluorescent techniques. Single-photon excitation comprises the transition of an electron in this fluorescent molecule to another molecular orbit, bringing the molecule from the ground state $\left(S_{0}\right)$ to the excited state $\left(S_{1}\right)$, as is depicted in the Jablonski diagram in Fig. 16.22. This transition is 


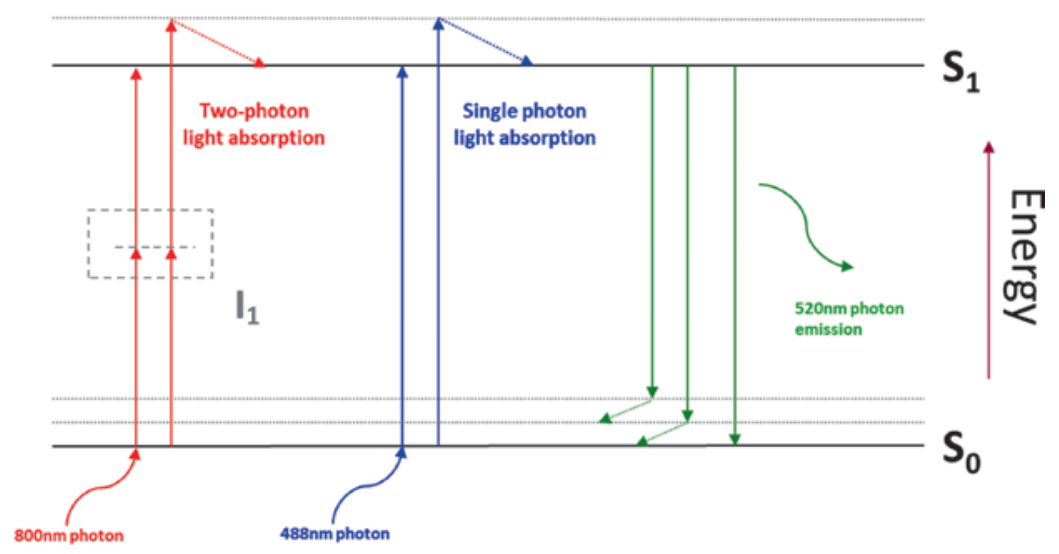

Fig. 16.22 Jablonski diagram showing single-photon excitation versus two-photon excitation. Single-photon excitation by, e.g., a solid-state laser in confocal setups uses a single photon to bring a fluorescent molecule from the ground state $\left(S_{0}\right)$ to the excited stated $\left(S_{1}\right)$, followed by

only transient, and upon return of the molecule to $S_{0}$, a fluorescent photon will be emitted. Since some energy is lost in between absorption and emission, the emitted photon has lower energy that the absorbed photon, shifting the emission to the red (Stokes shift).

Single-photon excitation as used in confocal microscopy, however, causes photodamage and phototoxicity, while its penetration depth into tissue is limited. Therefore, single-photon excitation is not the most suitable technique to study thick and living samples. The use of (near-)infrared (IR) light, as used in MP microscopy, can circumvent these problems. However, near-IR light is not capable of exciting the molecule by itself, since it lacks the energy to induce the transition from $S_{0}$ to $S_{1}$. As a result, the molecule will end up in the intermediate $I_{1}$ state. Only if two infrared photons arrive virtually simultaneously (within $10^{-18}$ s, i.e., one attosecond) at the molecule, they can together bridge the energy gap between $S_{1}$ and $S_{0}$. In a continuous laser beam, however, the probability of photons to arrive simultaneously at the molecule is virtually zero. By increasing the photon density, it is possible to increase this probability and thus to allow the transition from $S_{0}$ to $S_{1}$ and, thus, perform deeptissue fluorescence imaging. To achieve this, a pulsed laser is used (temporal confinement, fluorescence emission upon return of the electron from $S_{1}$ to $S_{0}$. Fluorescence emission can however also be achieved by two-photon excitation, in which two photons are required for transition from the ground state $\left(S_{0}\right)$ to the excited stated $\left(S_{1}\right)$ via the intermediate $\left(I_{1}\right)$ state

Fig. 16.23a), which emits femtosecond pulses with a high photon density at a high frequency $(80 \mathrm{MHz})$. Even under these prerequisites, effective two-photon excitation will happen only in the focal point (spatial confinement, Fig. 16.23b), as the probability of two-photon excitation decreases with a power of two with increasing distance to the focal point.

Especially in the recent $10-15$ years, MP microscopy has been applied more frequently. At first, application was especially focused on deeptissue imaging, since MP microscopy is one of the techniques which allows imaging up to $1 \mathrm{~mm}$ $(1000 \mu \mathrm{m})$ in, e.g., brain tissue, without requiring tissue sectioning or extensive tissue handling and/or fixation.

MP microscopy has several advantages over confocal microscopy. The most important hereof are the use of a pulsed laser system and the use of higher excitation wavelengths. The use of a pulsed (femtosecond) laser causes less photobleaching and phototoxicity. This is caused by the fact that light of higher wavelengths is less absorbed by, and as a consequence interacts less with, the tissue itself. The reduction in photobleaching can be explained among others by the reduction in out-of-focus excitation due to the spatial confinement as well as by the relative reduced time of excitation of the fluorophore by 


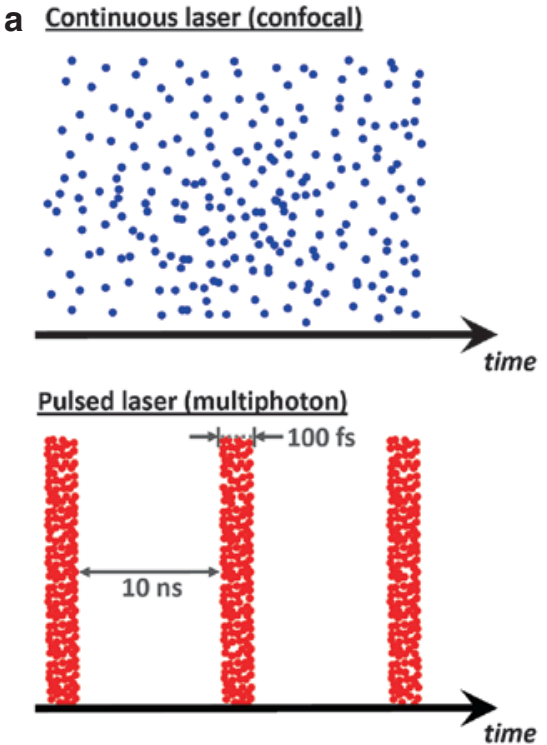

Temporal confinement b

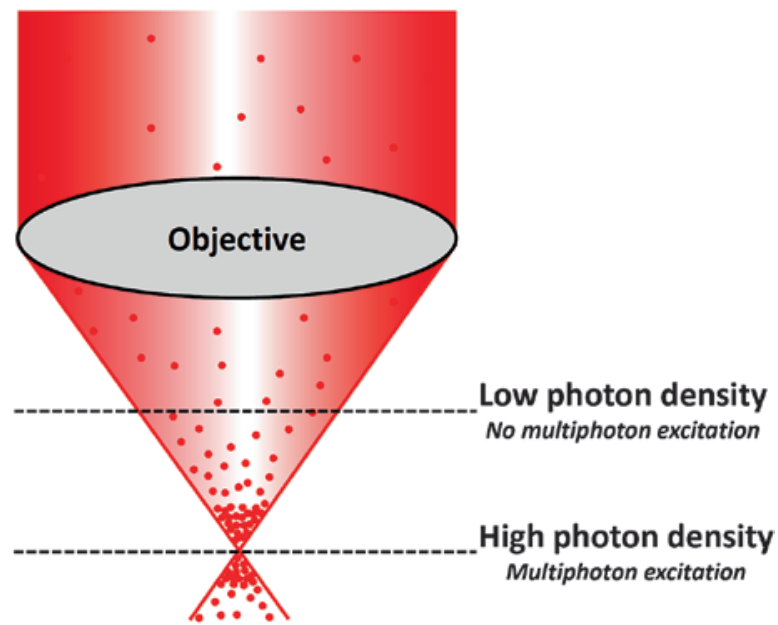

\section{Spatial confinement}

Fig. 16.23 Temporal and spatial confinement required for achieving fluorophore excitation in multiphoton microscopy. By temporal confinement, photon density is increased by creating narrow photon-dense pulses. Spatial

the temporal confinement compared to continuous excitation in regular confocal systems. In addition, excitation only occurs in focal point (i.e., no out-of-focus excitation as in confocal microscopy). Additionally, since there is no absorption in the out-of-focus plane, a higher penetration depth is achieved compared to confocal microscopy (Fig. 16.24).

The use of higher (near-)infrared light wavelengths also has distinct advantages. Firstly, it results in a higher penetration depth as discussed above, and, since these wavelengths are less damaging, they are very suitable for vital imaging. Moreover, since the two-photon excitation spectrum of many fluorescent probes is broader than its single-photon counterpart, simultaneous excitation of multiple probes often occurs as a result of offpeak excitation of these fluorophores using (near-) infrared light wavelengths. This may however also include excitation of autofluorescent molecules.

By combining the characteristics mentioned above with methods to allow high-speed image acquisition, intravital MP microscopy can be confinement is caused by the focusing capacity of objective lenses, where, as a result, the photon density is solely high enough in the focal plane of the specimen

achieved. The two most well-established ways of achieving high-speed imaging are resonant scanning and multibeam-based imaging (Nielsen et al. 2001; Fan et al. 1999; Niesner et al. 2007). In resonant scanning, high scanning speeds are achieved by the use of resonant galvanometer mirrors, which can oscillate with a frequency up to $12 \mathrm{kHz}$. The high speed, however, comes at the cost of increased noise levels, which favor the use of better detectors which have as little noise as possible. An alternative is a multibeam approach, in which the excitation laser beam is split into multiple (up to 64) beams which can simultaneously scan the specimen, allowing a faster scanning time. Here however, there is a loss of resolution with increasing penetration depth. Alternatively, triggering based on the cardiac and/or respiratory cycle can be used as a means to compensate for tissue motion, allowing acquisition of stable images in time but at the cost of temporal resolution.

In recent years, the applications of MP-based IVM have expanded greatly, and MP microscopy 

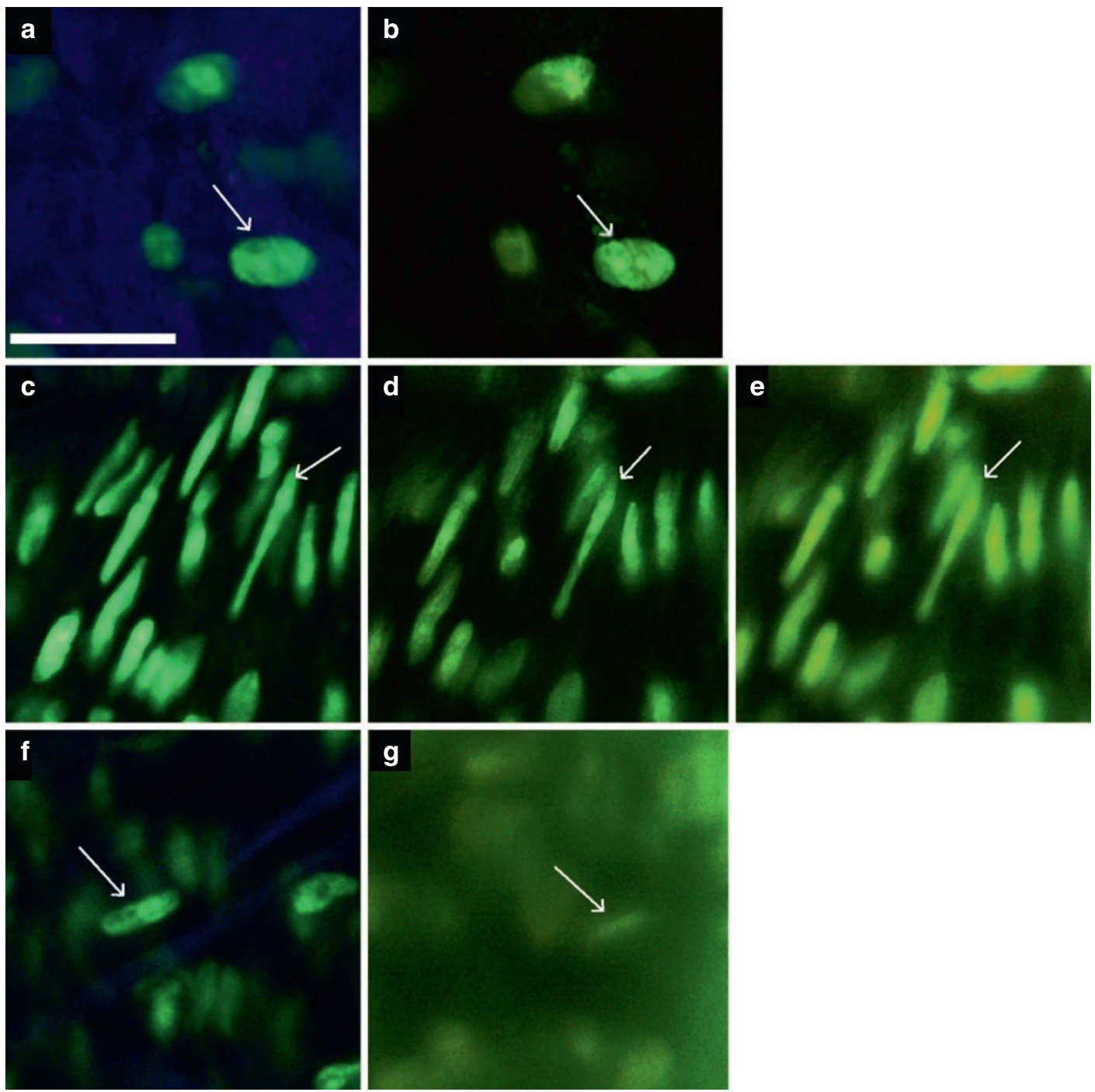

Fig. 16.24 Effect of penetration depth on the effective spatial resolution obtained with multiphoton microscopy (a, c, f) and confocal microscopy (b, d, e, g) as studied in a mouse carotid artery of which cell nuclei were labeled (green). Images are obtained at different depths: a, b, $15 \mu \mathrm{m}$ depth; c-e, $40 \mu \mathrm{m}$ depth; and $\mathbf{f}-\mathbf{g}, 80 \mu \mathrm{m}$ depth. Due to out-of-focus fluorescence in confocal microscopy,

has become a powerful technique for studying dynamic processes in, e.g., oncology, immunology, neuroscience, gastroenterology, urology, and cardiovascular research, as is depicted below.

\subsubsection{Cell Trafficking and Hemodynamics}

Similar to classic intravital imaging, MP IVM imaging has been used to study live process, such the effective resolution deteriorates far more with increasing depths as compared with MP microscopy. Confocal images obtained with optimal pinhole settings except for e in which the pinholes were fully open (Figure is adapted from van Zandvoort et al. (2004) and reprinted with permission)

as vascular permeability, leukocyte adhesion and transmigration, blood flow, shear rate, and hematocrit measurements in vivo. Significant progress has been made to allow imaging and quantification hereof. Nowadays, studies have been realized which are able to assess functional parameter in an entire tissue volume (Kamoun et al. 2010). By applying differential line scanning techniques, this can be achieved at several locations 

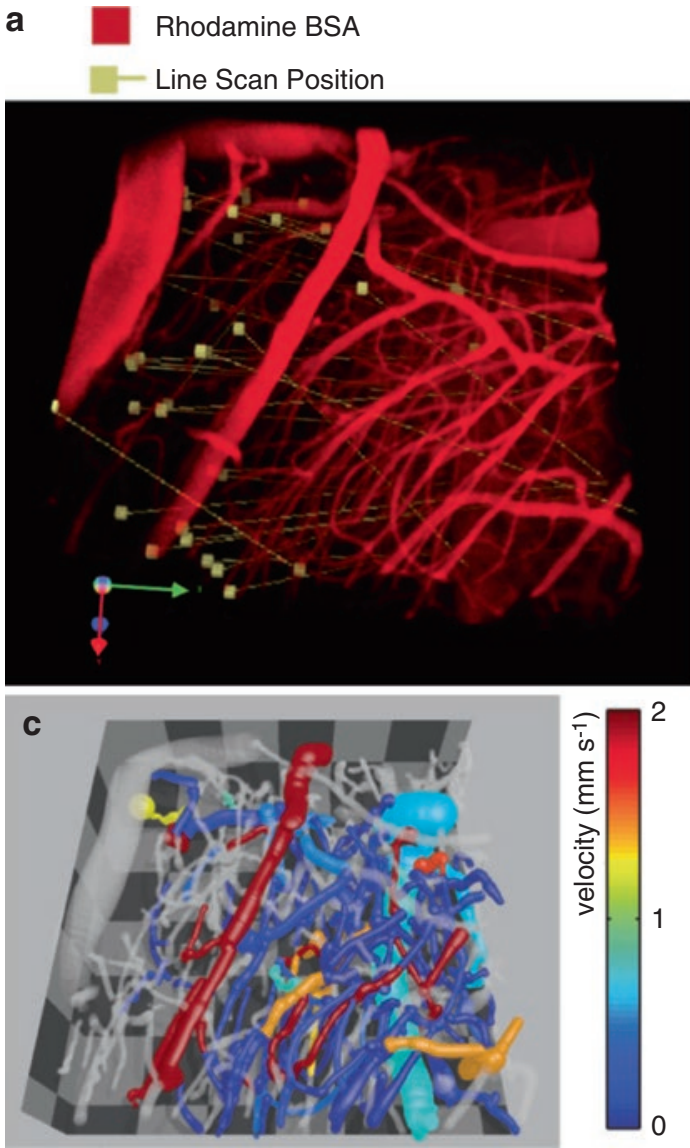

Fig. 16.25 Labeling both the cell-free blood volume and erythrocytes, it is possible to measure blood flow velocities in whole samples, e.g., the brain vasculature in a mouse, by using line scanning techniques (a). After image processing, involving post-processing of the acquired

at the same time, allowing the generation of velocity maps and comparing the functional makeup of a vascular bed (Fig. 16.25). More recently, this technology has even been applied in tissues suffering from substantial motion artifacts, such as atherosclerotic carotid arteries (Rademakers et al. 2013), in which functional parameters of microvasculature surrounding the atherosclerotic lesion were assessed (Fig. 16.26). Moreover, heterotopic beating heart models have been studied using MP-based IVM, allowing intravital imaging of leukocyte transmigration in the beating heart (Li et al. 2013b).

Alternatively, by applying triggering for the cardiac and respiratory cycle, image resolution can be improved. When the residual tissue motion

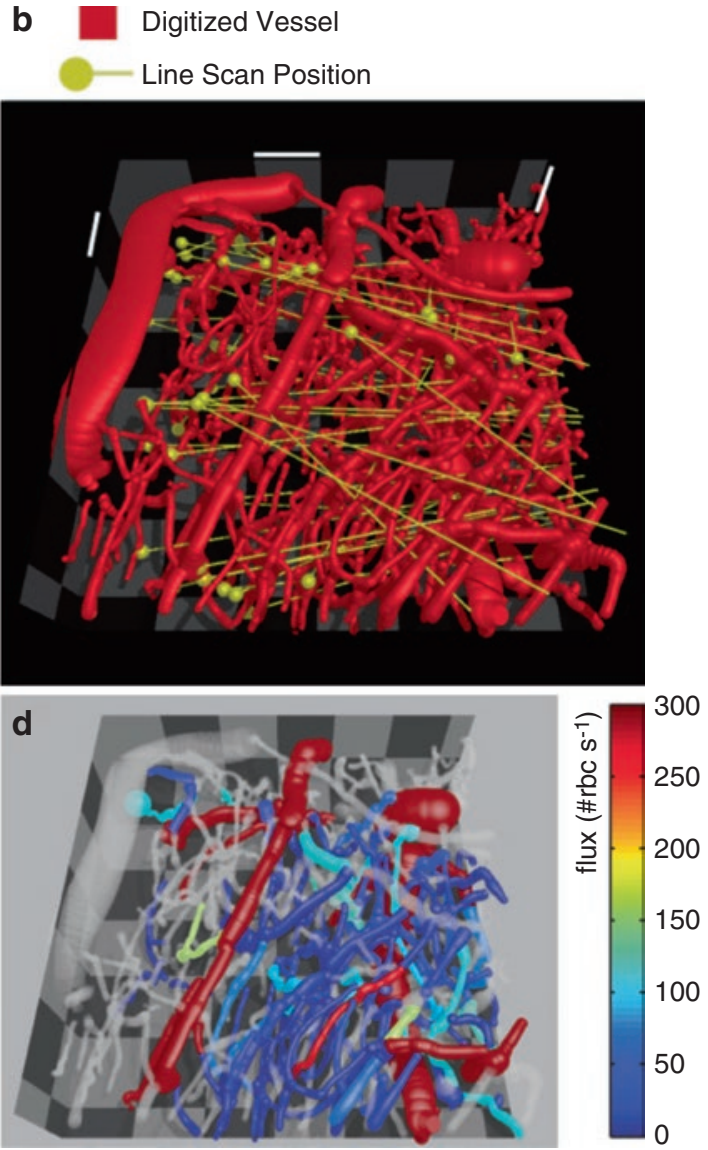

images (b), velocity maps (c), and maps revealing erythrocyte fluxes (d) within the vasculature can be constructed (Adapted from Kamoun et al. (2010); reprinted with permission)

can then also be reduced, as was proposed recently for carotid artery imaging, spatial and temporal resolution are even high enough to permit 4D imaging, i.e., the acquisition of 3D images in time (Fig. 16.27) (Chevre et al. 2014).

\subsubsection{Cell Tracking and Photoactivation}

As part of intravital MP, new applications for dedicated cell tracking have been developed, inspired by developments of photoactivatable fluorescent probes (Shaner et al. 2007). These probes allow otherwise non-fluorescent proteins to be specifically activated in a certain cells in a piece of tissue or organ, after which these cells can be specifically traced. The great advantage of 

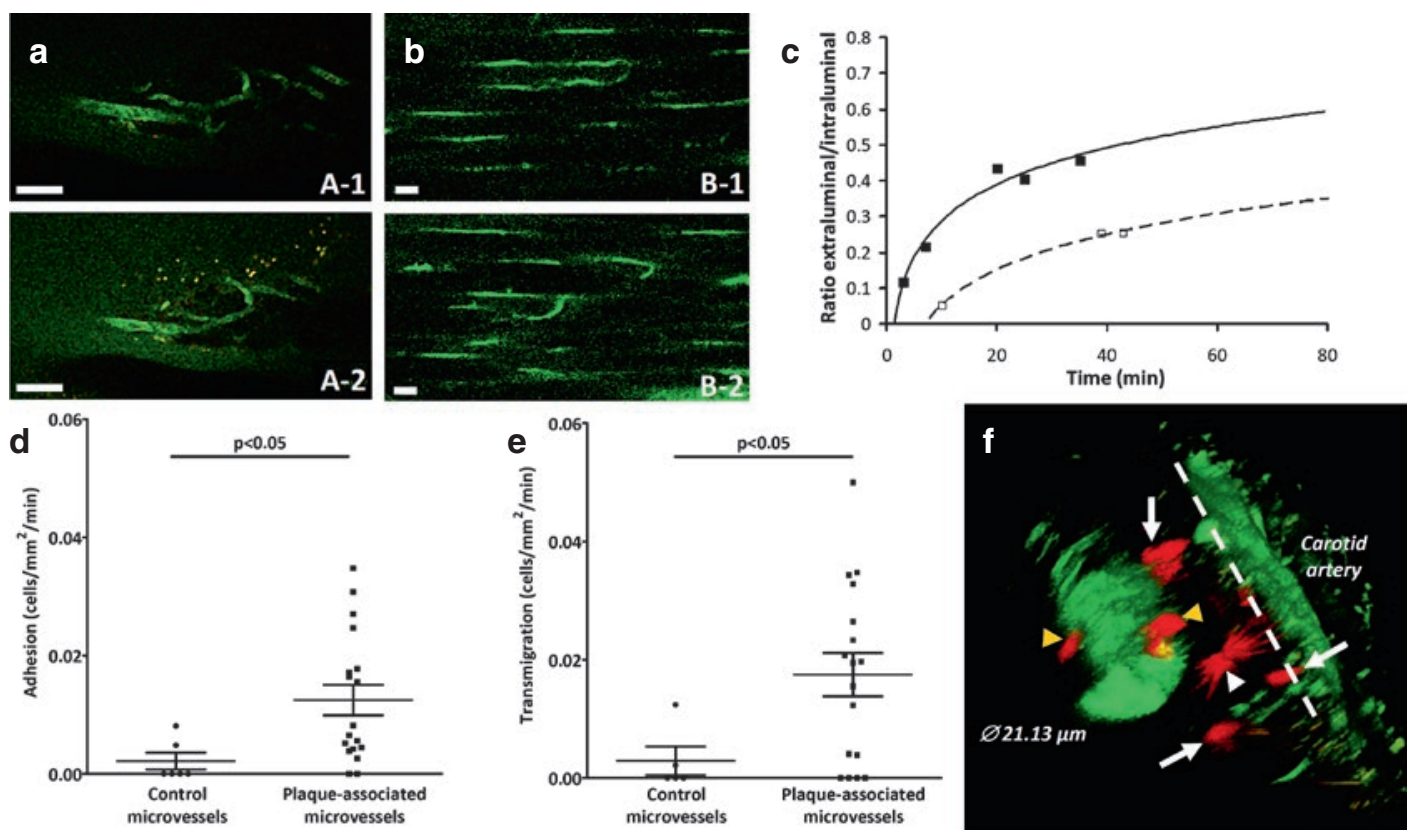

Fig. 16.26 Functional properties of the microvasculature can be studied in vivo in tissues suffering from substantial motion artifacts, such as atherosclerotic carotid arteries, by using resonant scanning MP-based IVM. Here, microvascular permeability for a $70 \mathrm{kDa}$ dextran (green, a,b) was studied in local (a) and control (b) microvascular tissue, revealing an increased permeability in the local microvasculature in time (c). Moreover, leukocyte adhesion and transmigration could be assessed $(\mathbf{d}, \mathbf{e})$, with additional in situ z-scans allowing 3D study (f) of the leukocyte $(r e d)$ transmigration process through the microvascular endothelium (green) (Adapted from Rademakers et al. (2013); reprinted with permission)

activity, vascular surface area and volume, or vascular permeability. As such, functional parameters can be quantified which can be used to assess efficacy of interventions. Similarly, network formation and functionality can also be implemented in different biological systems, e.g., neuronal networks (Eichhoff and Garaschuk 2011; Grewe et al. 2010; Gobel et al. 2007).

\subsubsection{Cellular Dynamics}

Another development is Förster resonance energy transfer (FRET) imaging using MP microscopy, although FRET imaging has already been a frequently used tool in confocal microscopy. The basis of FRET imaging relies on a FRET pair, i.e., two fluorescent proteins, which can act as a donor-acceptor pair. In such a FRET pair, the fluorescent protein with the lowest emission wavelength will be excited. When the two fluorescent proteins are in close proximity, their electron orbits overlap, which causes direct energy 

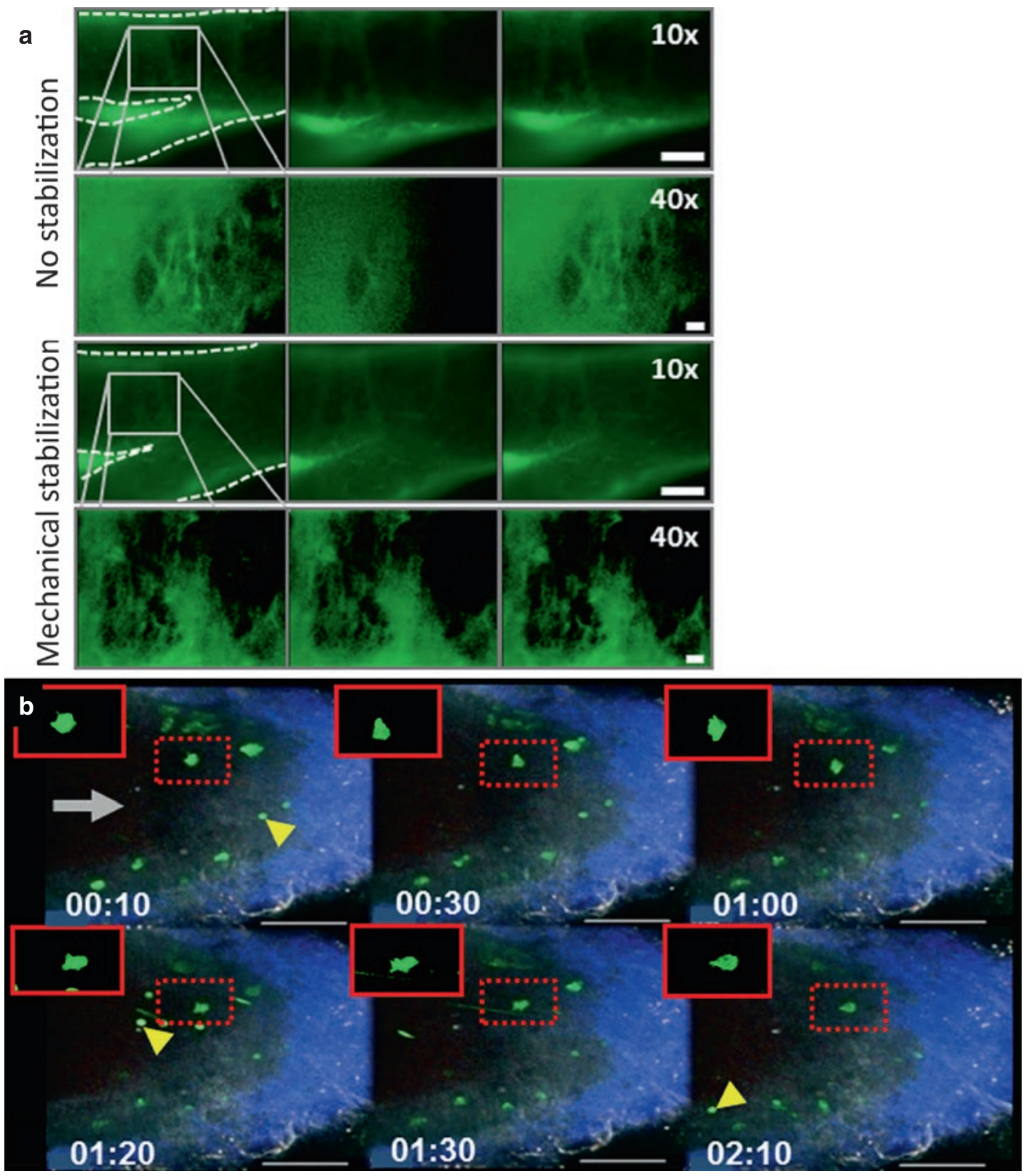

Fig. 16.27 Using triggered image acquisition and mechanical tissue stabilization, it is possible to greatly reduce the motion artifacts occurring during intravital imaging, hereby greatly enhancing image resolution (a). Such methods also allow imaging of tissue Z-stacks in time, i.e., 4D imaging. Maximum projection of time series shows the movement $\mathrm{GFP}^{+}$myeloid cells within a murine carotid artery (b) (Figure adapted from Chevre et al. (2014) and reprinted with permission)

nique is well suited for studying receptor-ligand binding, protease/kinase activity, and other molecular interactions.

FRET imaging has been applied in isolated vital tissue (Kapsokalyvas et al. 2014), but are in close proximity to each other, this tech- 


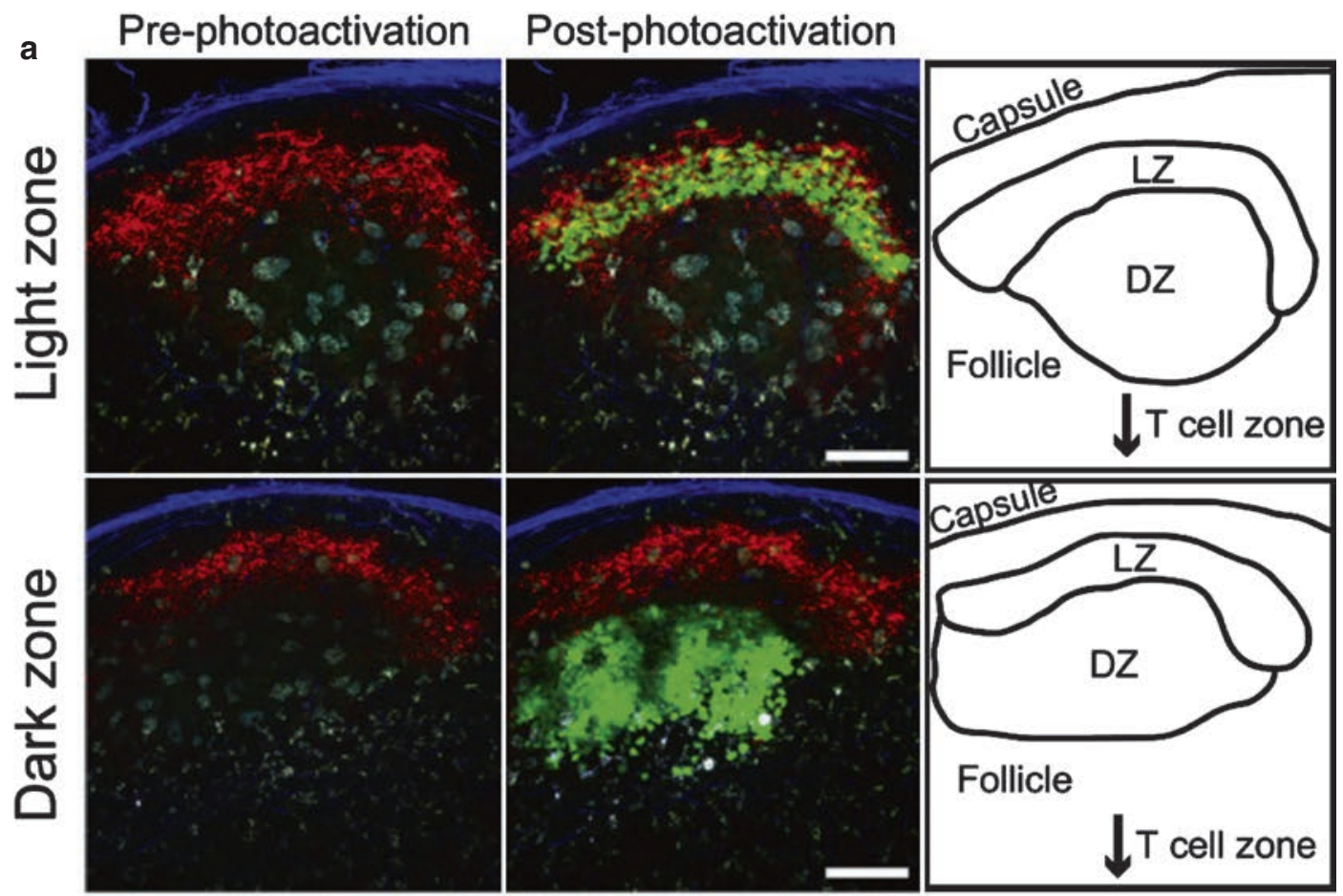

Collagen NP-tdTomato (FDC) Photoactivated PA-GFP
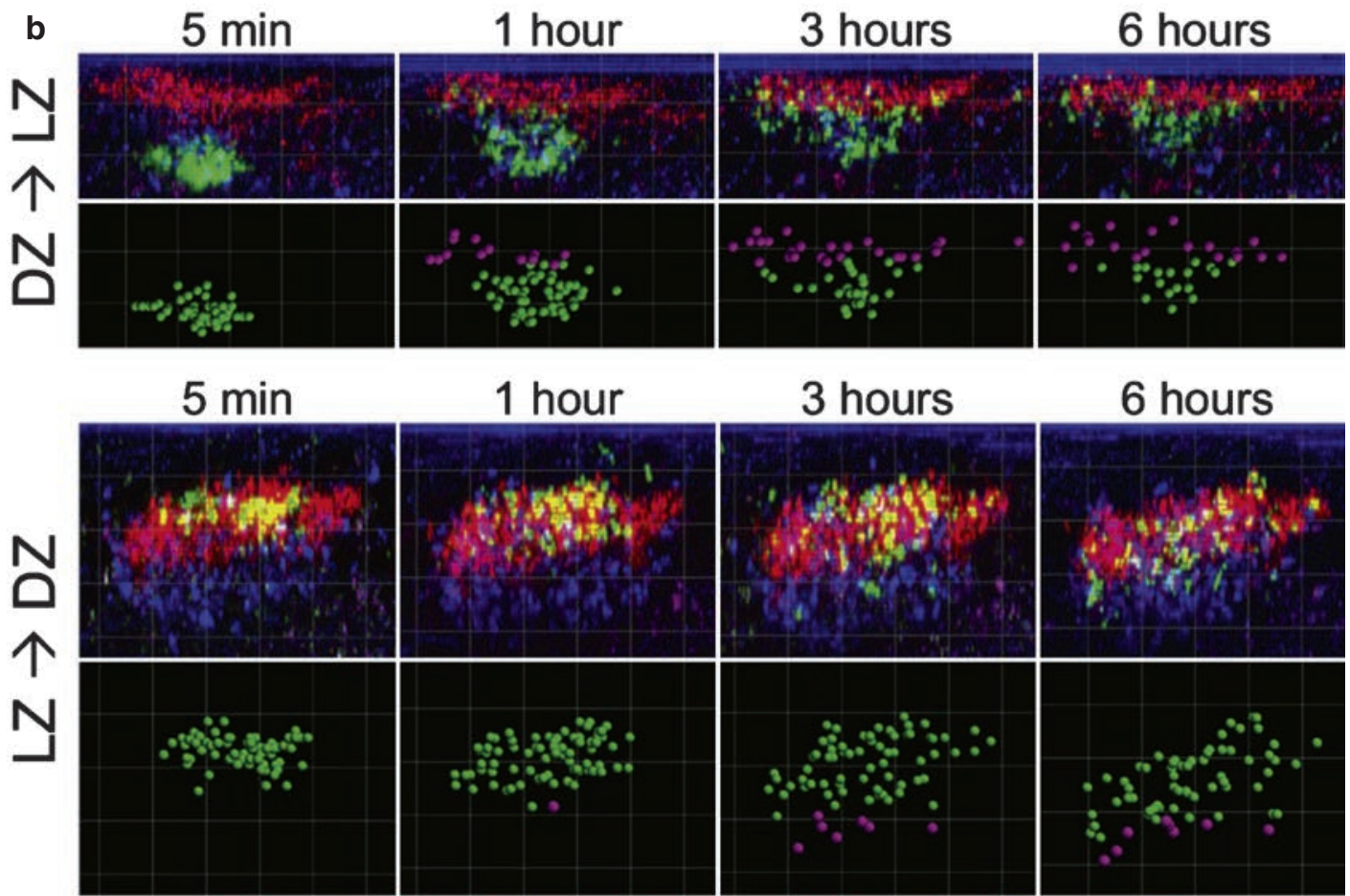

Channels: Photoactivated GC cells FDC network (NP-tdTomato) Collagen/CFP Spots: Photoactivated cells in original zone Photoactivated cells in opposite zone 
recently FRET imaging has been implemented in intravital MP microscopy as well. FRET imaging in a multiphoton microscope can be performed either by measuring differences in fluorescent donor lifetime upon FRET activity or simply by measuring the emission signal of the FRET acceptor molecule relative to the emission signal of the donor molecule (i.e., ratio imaging), the latter of which is used in intravital FRET imaging. By intravital FRET, it is possible to study molecular interactions in tissue in vivo and has, e.g., been used to establish kinase activity of Erk during neutrophil recruitment under inflammatory conditions in mice (Fig. 16.30) (Mizuno et al. 2014).

Other examples of studying cellular dynamics are determining calcium fluxes. This technique is used, e.g., in neuroscience, to determine neuronal activity (Mittmann et al. 2011), but nowadays these experiments are also being applied to other organs like the kidney, in which podocyte calcium fluxes were determined in vivo (Burford et al. 2014).

\subsubsection{Lineage Tracing}

In the last years, novel animal models, genetic constructs, and probe developments have led to the possibility of mapping cell fate (or lineage tracing) by IVM. Here, also photoactivatable or photo-switchable probes have been used, while other studies rely on the Brainbow approach (Mahou et al. 2012) to achieve this. The principle of Brainbow technology is based on the stochastic expression of multiple copies of constructs which code for different fluorescent proteins (Livet et al. 2007). As such, a multitude of color combinations can be achieved, and this technique has been used especially in neuroscience settings, where individual neurons and their mutual connections were studied (Fig. 16.31). Similar approaches have been derived from the Brainbow principle, including RGB marking (Weber et al. 2011) or generation of so-called Confetti mice (Schepers et al. 2012), and have introduced these lineage tracing approaches in other fields of research as well.

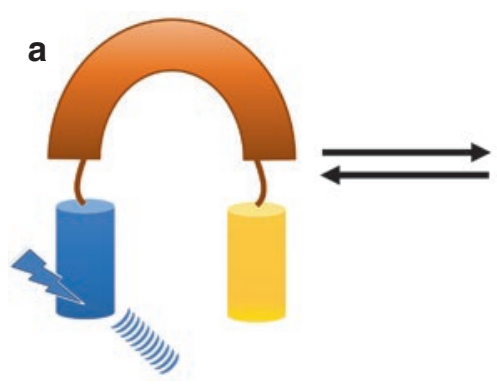

Fig. 16.29 FRET imaging is based on fluorescent proteins, e.g., cyan fluorescent protein (CFP, blue) and yellow fluorescent protein (YFP, yellow), which are linked together by a linker domain (a). Upon conformational changes (e.g., receptor activation), the two fluorescent proteins will come into close proximity, and energy trans-

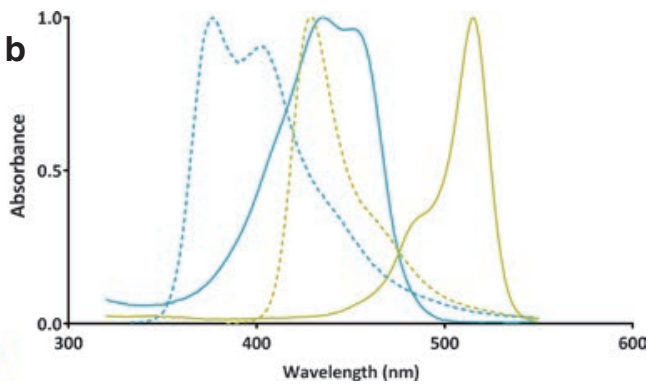

fer is possible between the two fluorescent proteins. Therefore, it is necessary that the emission wavelength (b, solid blue line) of the donor (i.e., CFP) overlaps with the excitation wavelength (b), dotted yellow line) of the acceptor (i.e., YFP)
Fig. 16.28 By using photoactivation in specific regions within a tissue, it is possible to study cellular dynamics between different tissue compartments, e.g., cell trafficking between the light (LZ) and dark zone (DZ) within the germinal centers of lymph nodes (a). After photoactiva- tion, computational analysis can determine the movement of cells in time from DZ to LZ and vice versa (b) (Figure adapted from Victora et al. (2010) and reprinted with permission) 


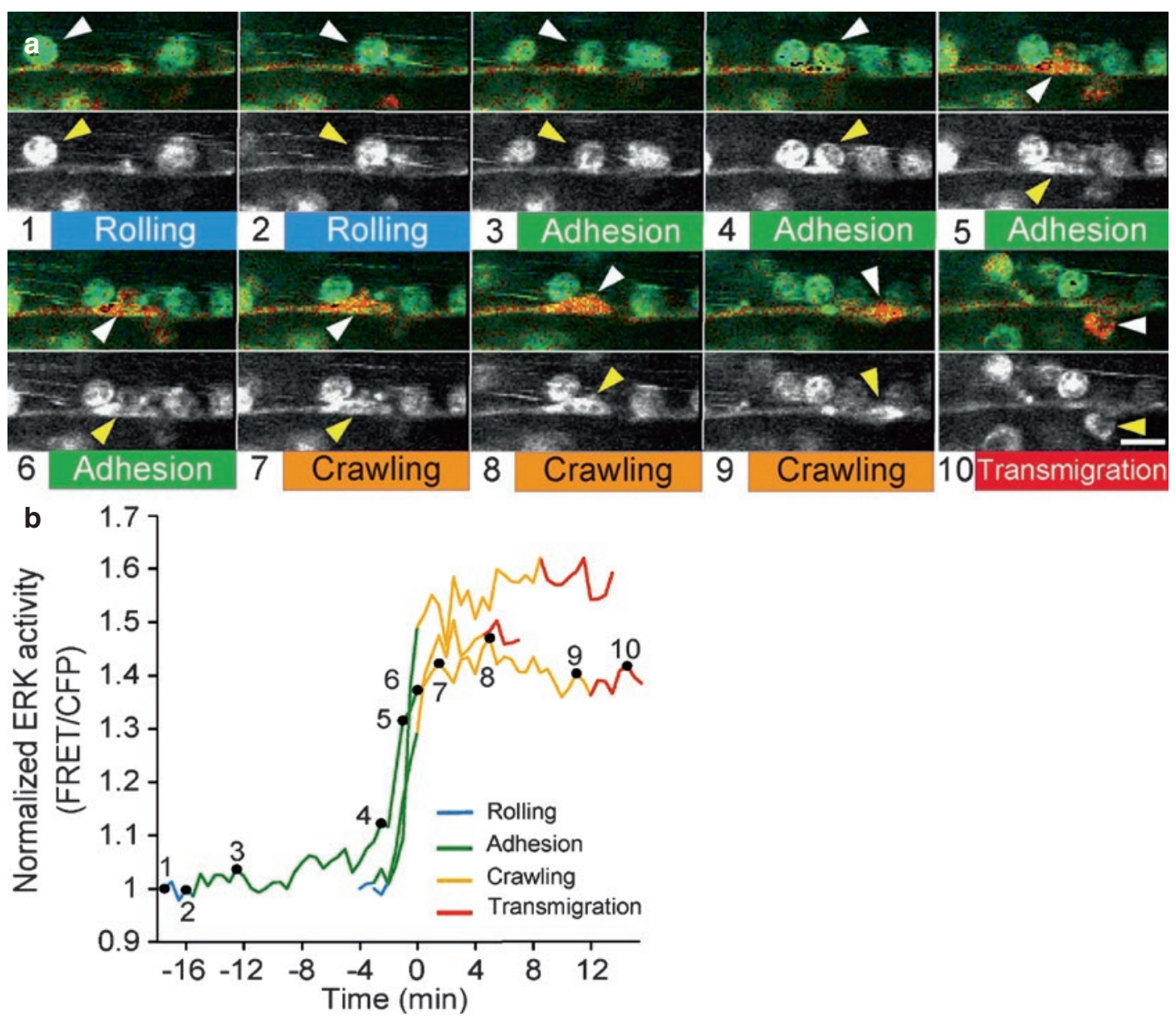

Fig. 16.30 FRET imaging has also been applied in IVM studies in mice, e.g., for studying kinase activity during neutrophil recruitment from the circulation to the intestine in a model of intestinal inflammation (a). These data can

\subsubsection{Perspectives and Considerations}

In all, the field of intravital imaging has undergone considerable changes, even though to date, even the classical intravital approaches are still being used to some extent. The type of intravital microscopy one would want/need to use will depend on several factors (Table 16.1). MP-based IVM on the one hand can give an integrated approach of multicolor, deep-tissue imaging, but the equipment is far more expensive and requires considerable technical expertise. Moreover, often large datasets are acquired, which require substantial data processing like image deconvolution, because of the increased be quantified, giving a measure of kinase activity during the different phases of neutrophil recruitment (b) (Figure adapted from Mizuno et al. (2014) and reprinted with permission) axial point spread function at higher wavelengths and increased depth in the tissue. Also data analysis in these larger datasets can be cumbersome. The classical techniques on the other hand are generally cheaper (depending on the specific setup) and easier to use and in many cases will be able to answer the research question at hand. The choice for the exact type of intravital imaging will therefore depend greatly on the research question at hand, as well as on the tissue/organ studied.

In line with these considerations, several other factors will influence the choice for intravital imaging, not unimportantly the availability of additional tools, e.g., adequate fluorescent probes, labeling strategies, or animal models. 

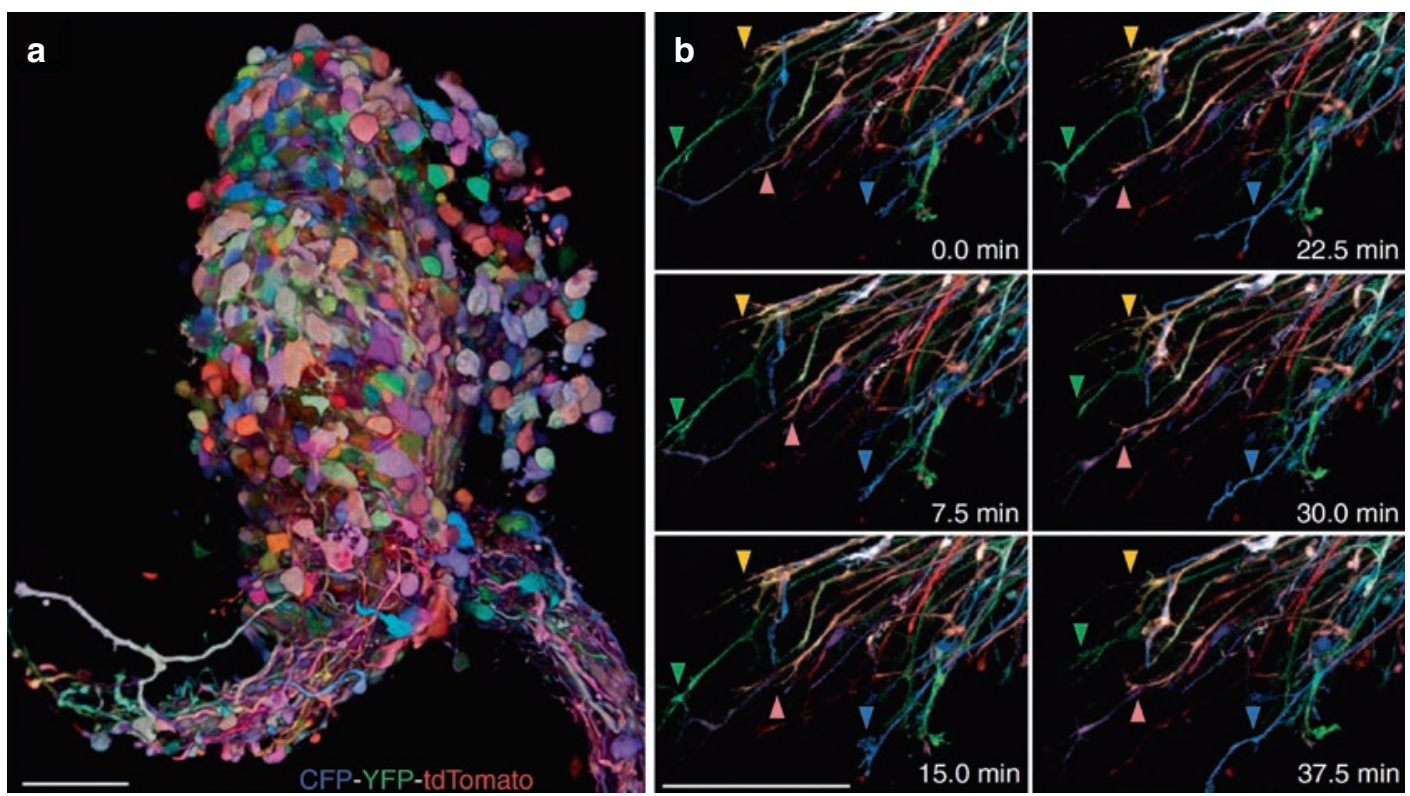

Fig. 16.31 By using a Brainbow approach, stochastic expression of multiple copies of constructs which code for different fluorescent proteins will lead to a multicolor mosaic. The color of a cell is dependent on the expression of these proteins, e.g., in the embryonic neural tube (a). In time, the growing motor neurons can be imaged (maximum intensity projections), allowing intravital lineage tracing of which cells induce axonal outgrowth (b) (Figure adapted from Mahou et al. (2012) and reprinted with permission)

Table 16.1 Comparison of the different IVM techniques with regard to acquisition speed, lateral and axial resolution, depth penetration into tissue, and possibility of multicolor imaging

\begin{tabular}{|c|c|c|c|c|c|}
\hline & Acquisition speed & Lateral resolution & Axial resolution & Depth penetration & Multicolor imaging \\
\hline Brightfield & ++ & + & -- & -- & -- \\
\hline \multicolumn{6}{|l|}{ Fluorescence } \\
\hline Widefield & ++ & ++ & -- & -- & $+1-$ \\
\hline Confocal & + & ++ & ++ & $+/-$ & + \\
\hline Spinning disk & ++ & ++ & $+1-$ & - & $+1-$ \\
\hline Multiphoton & + & ++ & $+/++$ & ++ & ++ \\
\hline
\end{tabular}

Based on Megens et al. (2011)

“-_" very low, “-” low, “+/-” moderate, “+” good, “++" very good

Especially in multiphoton setups, in which multiple fluorophores are excited simultaneously, there is a need for fluorescent probes with relative narrow emission spectra in order to distinguish between the fluorophores. Novel probes like quantum and carbon dots present promising tools (Douma et al. 2009). Next, the ability to label specific structures is an important issue (Progatzky et al. 2013). Usually, the vasculature and cells in circulation can be stained easily by intravenous injection of specific fluorescent antibodies, but other cell types are more challenging.
The development of novel transgenic reporter mice as well as, e.g., FRET-sensor knock-in mice will provide an elegant tool for this purpose.

Yet, one of the greatest challenges still lies in achieving high-resolution imaging in vivo in various tissues. In fluorescence-based IVM, autofluorescence can significantly hinder proper image acquisition and requires careful consideration when selecting fluorescent probes. Worth mentioning, autofluorescence can also provide a means for label-free imaging. Imaging of, e.g., $\mathrm{NAD}(\mathrm{P}) \mathrm{H}$ has been used to assess mitochondrial 
function in skeletal muscle in vivo (Rothstein et al. 2005). Also processes like second and third harmonics generation (SHG and THG, respectively) can be used to study different cell types, e.g., erythrocyte detection by THG, which can be used for label-free determination of hemodynamic factors (Dietzel et al. 2014).

Beyond autofluorescence, scattering properties of the tissue will also hinder image acquisition or limit penetration depth. Even though the inherent properties of tissue are not easy to alter, ex vivo attempts to induce optical clearing of tissue have proven to be quite successful, especially in brain tissue (Chung and Deisseroth 2013). First attempts to achieve optical clearing in vivo have been successful in, e.g., skin imaging using optical coherence tomography in rats (Genina et al. 2014), although application on an organ level in vivo - especially without altering function of the organ - remains tentative. Improvement of optics, like the development of graded index (GRIN) objectives, may however offer another way of improving imaging and even allow fiberoptic-based imaging, like intravascular or intracolon imaging. Also, applications of these objectives in brain and kidney imaging in vivo have given promising results (Levene et al. 2004; Li and Yu 2008).

Taken together, intravital imaging is a vital tool for studying dynamic process in small animal models. Depending on the research question, different variants of IVM may be used to answer this question. Ongoing and novel technical improvements on intravital imaging equipment as well as probes and animal models will provide valuable tools for many fields of research in the years to come.

\subsection{Optoacoustic Imaging}

Daniel Razansky

\subsubsection{Introduction}

The imaging depth limitation of optical microscopy related to light scattering in living tissues can be generally overcome by means of optoacoustic (OA) techniques (Razansky et al. 2012). By detecting pressure waves induced via transient absorption of short high-energy laser pulses, OA methods have recently enabled high-resolution imaging with optical contrast at depths of several millimeters to centimeters (Wang and $\mathrm{Hu}$ 2012), orders of magnitude deeper than possible with modern microscopy. OA sensing and imaging draw its roots from the discovery of the photophone by A. G. Bell and his assistant C. S. Tainter in 1880, which the inventors used as the first practical wireless telephony or, in fact, optical communication device (Tainter and Bell 1880). However, due to lack of appropriate laser sources and ultrasound detection technologies, utilization of optoacoustics to biomedical applications, such as spectroscopy or imaging, was first considered in the 1970s (Rosencwaig 1973) with first images from living small animals obtained in the past decade (Wang et al. 2003). By combining commercially available pulsed laser technology in the nanosecond range and sensitive acoustic detectors, it was shown possible to generate OA responses from tissue that carry spatially resolved spectroscopic information on the underlying absorption contrast (Ntziachristos and Razansky 2010). Nowadays, the terms optoacoustic and photoacoustic are equally used to describe the effect of acoustic wave generation by transient light absorption. Due to its hybrid nature combining optical excitation and acoustic detection, the OA imaging technology benefits both from the rich and versatile optical contrast and high (diffractionlimited) spatial resolution associated with lowscattering nature of ultrasonic wave propagation as compared to photon propagation (Wang and Hu 2012).

The ability to spatially resolve spectral information, i.e., location/size of unique tissue structures containing specific chromophores, provides added advantage of OA techniques. Rendering images at multiple excitation wavelengths and spectral unmixing allow for visualization of intrinsic tissue chromophores, such as melanin (Krumholz et al. 2011), oxy- and deoxyhemoglobin (Laufer et al. 2007), and lipids (Jansen 
et al. 2014). Multispectral optoacoustic tomography thus holds a great promise for functional and molecular imaging, which is highlighted by the broad range of emerging biological applications of optoacoustics, including cardiovascular imaging (Taruttis et al. 2010), monitoring of organ perfusion and pharmacokinetics (Buehler et al. 2010b), targeted studies of inflammation and arthritis (Beziere et al. 2014; Vonnemann et al. 2014), cancer detection and staging (Mehrmohammadi et al. 2013), neuroimaging (Nasiriavanaki et al. 2013), and dermatologic imaging (Ida et al. 2014).

\subsubsection{Principles of Optoacoustic Imaging}

Optoacoustic (OA) imaging is based on absorption of light radiation in tissue and conversion of the deposited energy into heat, which in turn results in thermal expansion and mechanical stress propagating in the form of pressure waves (Fig. 16.32).

Similarly to most optical imaging modalities, light in the visible or near-infrared spectrum $(400 \mathrm{~nm}-1200 \mathrm{~nm})$ is normally used for excitation of OA responses due to the relatively weak absorption of biological tissues in this spectral region. Of particular importance is the near-infrared spectral window, which allows light penetration of up to several centimeters into optically dense tissues (Ntziachristos et al. 2005). Alternatively, tissue can be excited with energy in the radiofrequency and microwave spectra (Razansky et al. 2010), which is however not included in the scope of this chapter. Large variety of chromophores is absorbing at the optical wavelengths, which further leads to a high contrast between different tissues with varying chromophore concentration. Figure 16.33 shows absorption spectra of the most dominant intrinsic absorbers in tissue in the visible and near-infrared range (Yao and Wang 2014). For biomedical applications, except for intrinsic chromophores (e.g., hemoglobin in its oxygenated and deoxygenated form, melanin, fat), extrinsically administered agents, such as

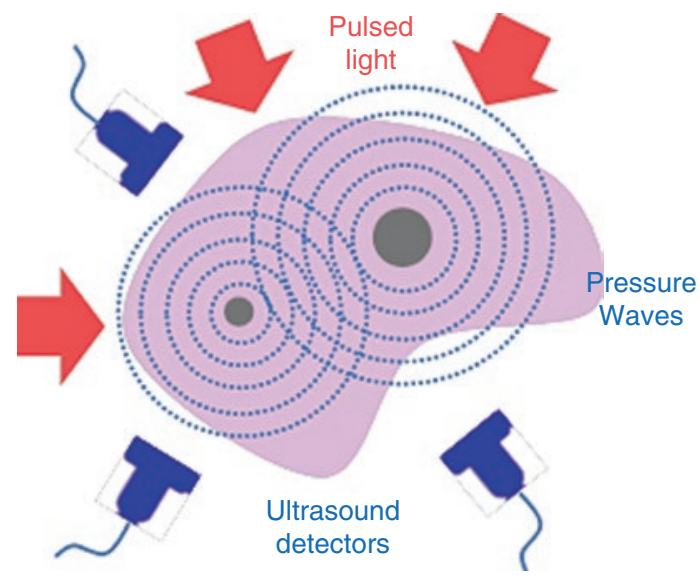

Fig. 16.32 Basic principle of optoacoustic imaging. Short pulses of light are absorbed by tissue absorbers, generating pressure (acoustic) waves propagating through the medium. The latter are recorded by ultrasonic detectors and used to form optoacoustic images by means of reconstruction methods
Fig. 16.33 Absorption spectral profiles of major intrinsic tissue chromophores responsible for generation of optoacoustic responses

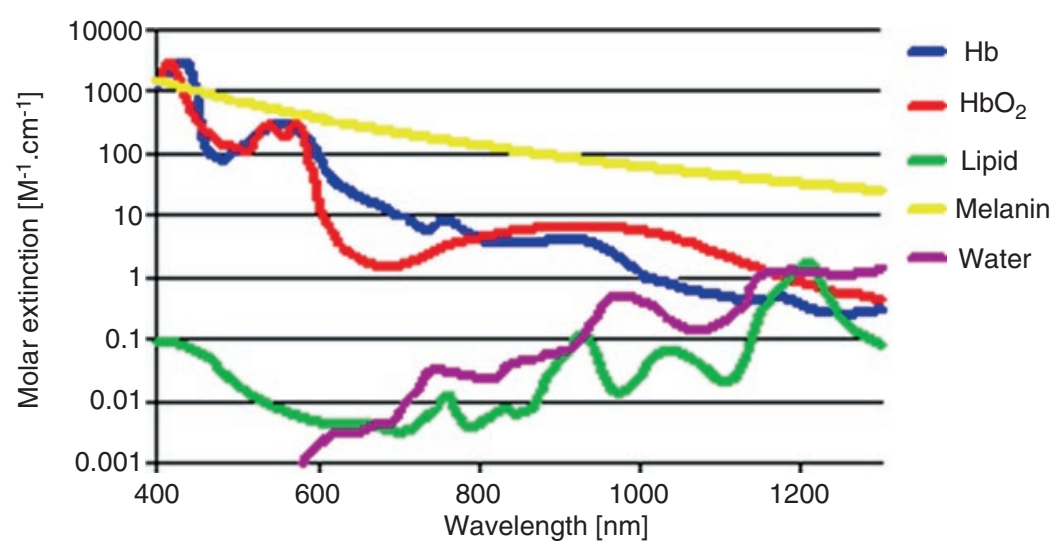


nanoparticles or fluorophores (Amiot et al. 2008; Tong et al. 2009; De La et al. 2008), or genetically encoded markers (proteins) (Razansky et al. 2009), can be additionally employed for contrast enhancement.

Vast majority of the modern OA imaging setups utilize lasers that emit ultrashort pulses in the nanosecond range, mainly due to good signal-tonoise performance and ease of parallelization. Under thermal and stress confinement conditions (Lutzweiler and Razansky 2013), usually assumed with respect to the duration of the laser pulse in this regime, the initially induced pressure distribution $p_{0}(r)$ is generally proportional to the distribution of the absorbed optical energy density $H(r)$ via

$$
p_{0}=\Gamma \cdot H,
$$

where $\Gamma$ is the dimensionless Grüneisen parameter describing the thermal and mechanical properties of the imaged tissue. Since $\Gamma$ it does not exhibit significant variations among different soft tissues, it is most often assumed to be constant at a constant temperature.

The initial goal of optoacoustic imaging and tomography is to retrieve the absorbed optical energy density inside the object. However, the generated pressure fields can normally only be measured outside the object, whereas propagation of pressure waves toward the detection point is described by the optoacoustic wave equation (Kruger et al. 1995). The way to obtain optoacoustic images therefore consists in reconstructing distribution of the energy density $H(r)$, i.e., solving or inverting the optoacoustic wave equation given the pressure variations measured around the imaged object.

For biomedical applications, it is the spatial distribution of the optical absorption coefficient $\mu_{a}(r)$ that is usually more directly related to the biochemical composition of the imaged tissue rather than the absorbed energy density. Both are AU17 related via

$$
H=\mu_{a} \cdot \Phi
$$

where $\Phi$ is the light fluence (or photon density) field. Although one may presume that $H$ it is a simple product, it in fact depends nonlinearly on the absorption coefficient $\mu_{a}$. This is because the light fluence generally depends on the underlying optical properties, including optical scattering and absorption coefficients. Thus, it is important for image quantification purposes to account for the light fluence since it may considerably vary as a function of depth.

\subsubsection{Multispectral Optoacoustic Tomography}

While reconstructing distribution of the optical absorption coefficient may provide valuable structural (anatomical) information on the underlying tissue contrast, it is the concentrations of different chromophores $c_{i}$, not the optical absorption coefficient $\mu_{a}$, that are mainly of interest from the biological point of view. For instance, in many applications, the blood-related absorption is of particular interest, providing valuable physiological or functional information. The relation between concentrations of the different chromophores and the optical absorption coefficient may be expressed via linear superposition, namely,

$$
\mu_{a}(\lambda)=\sum_{i} \varepsilon_{i}(\lambda) \cdot c_{i}+\mu_{\mathrm{BG}}(\lambda)
$$

where $\varepsilon_{i}(\lambda)$ are the wavelength-dependent molar extinction coefficients of the different intrinsic tissue chromophores or extrinsically administered agents and $\mu_{\mathrm{BG}}$ is the residual (background) absorption, which might also include noise. To differentiate between contributions of different chromophores, their distinct spectral dependence on wavelength can be assessed (Rosencwaig 1973; Kostli et al. 2000). This multiwavelength imaging approach is known as multispectral optoacoustic tomography (MSOT) or spectroscopic imaging (Laufer et al. 2007; Razansky et al. 2009; Sethuraman et al. 2008; Razansky 2012). The process of recovering $c_{i}$ from multispectral measurements is known as spectral unmixing. It can be combined with calculation of the light fluence or treated as a separate image processing step. 


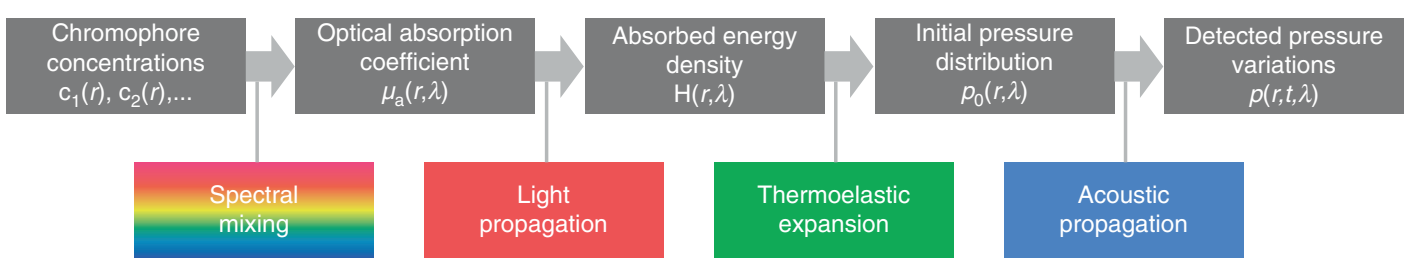

Fig. 16.34 Schematic representation of the sequence of generation and detection of optoacoustic signals

Due to versatility and wide availability of optical molecular agents, sensitive and accurate spectral processing to recover concentration of extrinsically administered agents may enable longitudinal molecular imaging studies. This can be done by resolving accumulation of agents with specific spectral signatures, such as targeted and activatable fluorescent molecular agents, nanoparticles, or genetic markers.

Figure 16.34 summarizes the main contributions to the OA signal generation chain, which need to be accounted for when developing accurate image reconstruction algorithms.

\subsubsection{Optoacoustic Imaging Instrumentation}

A typical optoacoustic setup consists of several key components. In the pulsed excitation mode, the tissue is illuminated by laser emitting monochromatic pulses of light with typical duration of some nanoseconds. For deep-tissue imaging applications, optical parametric oscillators are often used to provide a tunable wavelength in the spectrum of interest with pulse repetition rate in the order of a few tens of Hertz and per-pulse energies in the millijoule range. In optoacoustic microscopy and other superficial applications, where such high per-pulse energies are not required, other types of sources in the microjoule and nanojoule range are considered as well, including high repetition dye lasers (Song et al. 2009), laser diodes (Allen and Beard 2006b), and fiber lasers (Wang et al. 2011).

For tomographic imaging, the optoacoustically generated pressure profiles are captured with detectors surrounding the object. In comparison to ultrasound (US) imaging, OA signals are broadband (from several tens of $\mathrm{kHz}$ up to a $100 \mathrm{MHz}$ for small structures), while their amplitude is relatively low. Their frequency content is mainly dependent on the characteristic size of absorbers in the imaged volume. Due to the dominating low-frequency content of OA waves generated by common biological targets, utilization of physical or synthetic aperture focusing may turn inefficient. Moreover, while in US the focusing can be done in both transmission and detection, only the latter is relevant for OA. On the other hand, the optical absorption contrast may reach more than one order of magnitude for blood versus other tissues (Wang 2009) versus up to $10 \%$ contrast between soft tissue pulse-echo US imaging (Duck 1990). As a result of both inefficient focusing and high-absorption contrast, OA image formation using linear phased arrays and other focusing techniques suffers from severe out-of-focus artifacts, impaired contrast, image blurring, and overall lack of quantification abilities (Lutzweiler and Razansky 2013). Thus, in contrast to pulse-echo US, correct image reconstruction in OA imaging is ideally achieved by an unfocused detection of OA responses from as many tomographic viewing angles as possible around the imaged object.

Acoustic coupling between object and detector is usually ensured by water or coupling gel. For acquisition of spatially resolved data, either a single detector is scanned around/along the object or multiple detectors acquire the data in parallel. The latter further allows for fast data acquisition, e.g., rendering images from single laser shots. The signals are then pre-amplified and digitized by a fast data acquisition system. Figure 16.35 illustrates the broad variety of OA setups employed for small animal imaging, including optical-resolution microscopy (Hu et al. 2011), endoscopy 

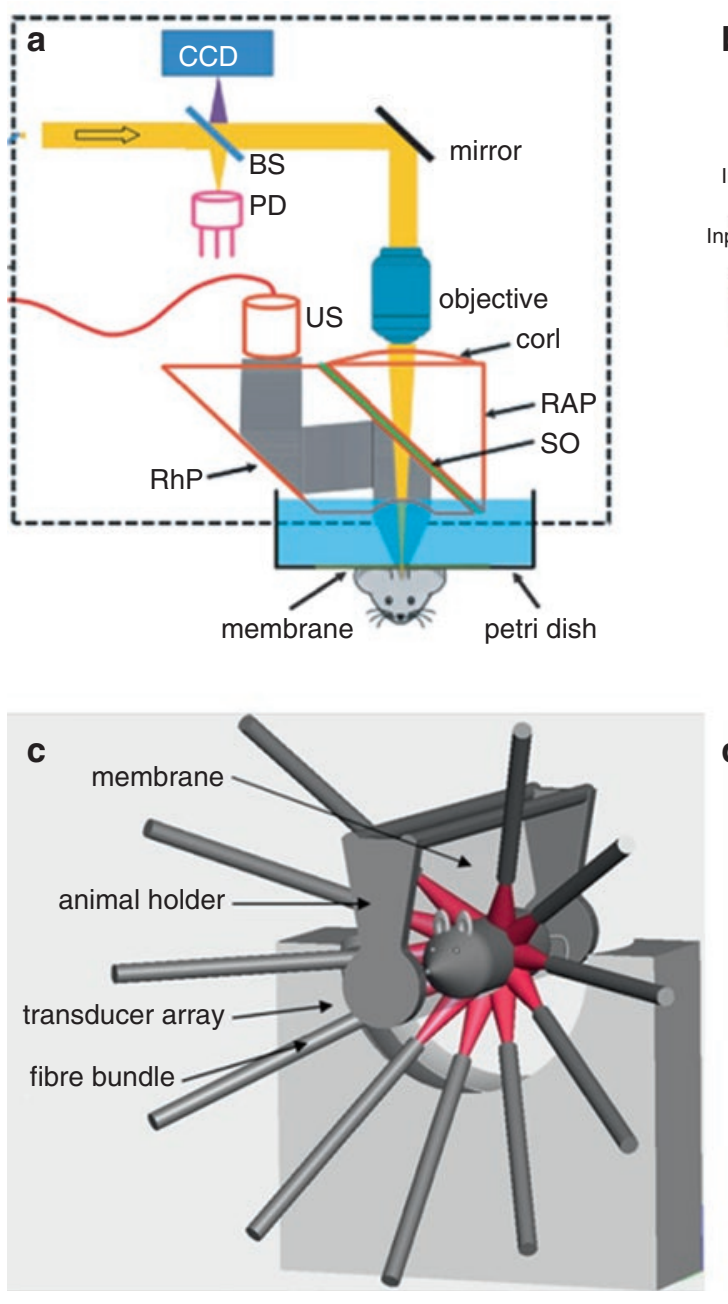

Fig. 16.35 Various types of optoacoustic imaging systems. (a) Optical-resolution photoacoustic microscopy (OR-PAM) scanner used for in vivo imaging of mice (Hu et al. 2011). Spatial resolution of the system in the lateral direction is determined by the size of the optical focus. Three-dimensional data is acquired by raster scanning of the optical beam along the imaged area. $B S$ beam splitter, $P D$ photodiode, CorL correction lens, RAP right-angle prism, $S O$ silicone oil, $R h P$ rhomboid prism, $U S$ ultrasonic transducer (central frequency $-50 \mathrm{MHz}$ ). The CCD is used to view the imaging region (Reprinted with permission from the Optical Society of America). (b) Combined endoscopic system for co-registered US and dual-wavelength functional OA imaging in vivo (Yang et al. 2012). The endoscope carries out circumferential sector scanning by rotating a scanning mirror, which reflects both the ultrasonic waves and laser pulses and enables static mounting of the associated illumination and ultrasonic pulse-generation detection units (Reprinted with permission from the Nature Publishing Group). (c)

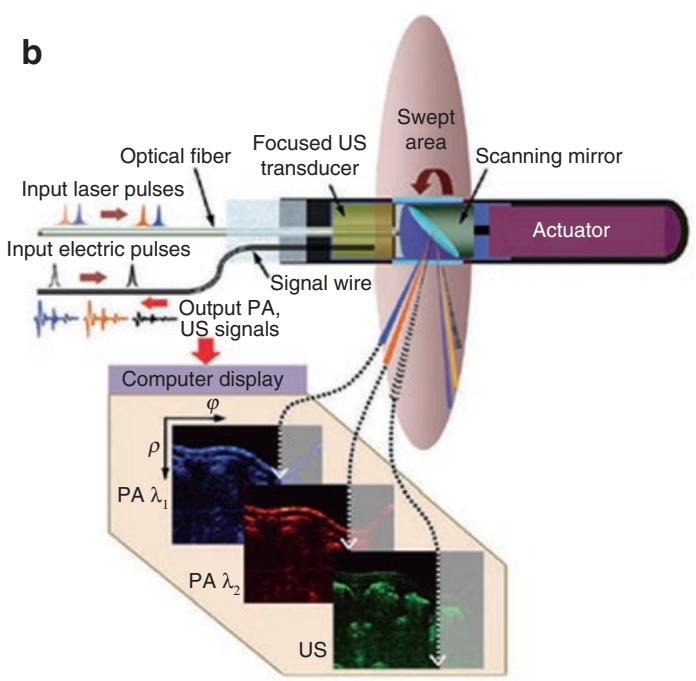

d

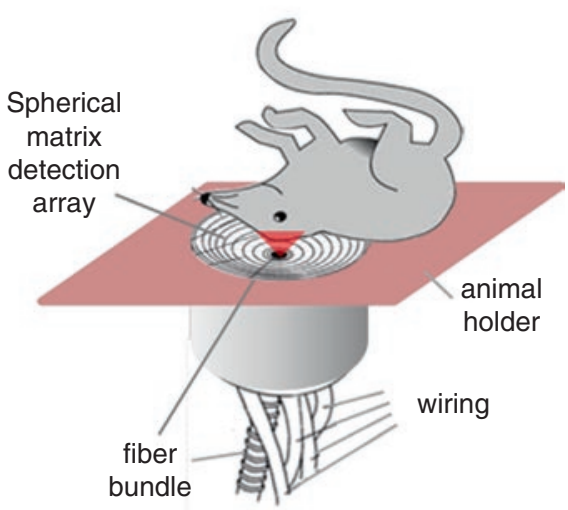

Schematic drawing of the real-time whole-body mouse scanner including the animal holder, illumination device, and transducer array (Razansky et al. 2011). A curved array of wide-band and cylindrically focused ultrasound transducers enables parallel data acquisition from the imaged cross-sectional plane. Optical fibers are used to homogeneously illuminate the object. By translating the animal holder, three-dimensional data can be acquired (Reprinted with permission from the Nature Publishing Group). (d) Real-time volumetric imaging system comprising of a spherical matrix ultrasound detection array with the laser illumination provided via a fiber bundle pulled through the center of the array. Rapid acquisition of multiwavelength data from an entire imaged volume, combined with real-time image reconstruction and spectral unmixing, provides four- and five-dimensional imaging capabilities by rendering volumetric unmixed images of individual chromophores in real time (Deán-Ben and Razansky 2014) (Reprinted with permission from the Nature Publishing Group) 
(Yang et al. 2012), cross-sectional whole-body tomography (Razansky et al. 2011), as well as real-time volumetric (4D) and 5D imaging systems (Deán-Ben and Razansky 2014).

\subsubsection{Small Animal Imaging Applications}

Due to its rich optical contrast and high spatiotemporal resolution, optoacoustics can deliver multiple types of contrast from deep tissues. Naturally, the best intrinsic tissue contrast at the visible and near-infrared wavelengths arises from highly absorbing hemoglobin; thus, blood vessels are clearly visible in the optoacoustic images. OA microscopy is thus often geared toward investigation of vascular structures and neovascularization, providing an excellent intrinsic contrast and a high spatial resolution in the order of some tens of $\mu \mathrm{m}$ and penetration of several millimeters into scattering tissues. In this way, disease-related anatomical changes can be visualized, e.g., development of tumor neovasculature (Laufer et al. 2012) (Fig. 16.36a). While images can be generated at a single wavelength, multiwavelength illumination and spectral processing are necessary for optimally identifying the unique spectral signatures of intrinsic tissue chromophores, such as hemoglobin, water, and lipids. It is therefore natural for OA imaging to volumetrically visualize blood oxygenation levels in living tissues by resolving the contribution from oxygenated versus deoxygenated hemoglobin. This functional imaging capability is showcased in Fig. 16.36b where oxygen saturation status of individual blood vessels was visualized with the optical-resolution microscopy method (Hu et al. 2011).

Preclinical whole-body imaging of small animals with multispectral optoacoustic tomography (MSOT) systems is yet another key application of OA imaging. These systems can provide tomographic images with a resolution in the order of hundred $\mu \mathrm{m}$ for depths between several millimeters to several centimeters. Possible applications include monitoring of tumor hypoxia, drug response, or molecular targets in biological model organisms (Herzog et al. 2012; Lozano et al. 2014). The ability of MSOT to visualize deep-seated fluorescent proteins with high resolution has been also demonstrated (Razansky et al. 2009). Figures 16.36e, f show example of a study on kinetics and biodistribution of near-infrared contrast agent in a living mouse (Taruttis et al. 2012). The uptake of a carboxylate dye in separate regions of the kidneys was tracked in real time over $30 \mathrm{~min}$ post its systemic injection. The images indicate different dynamics in different areas of the kidneys, suggesting that two dependent processes are being imaged, firstly the filtration of agent in the cortex and subsequently the excretion toward the ureter.

Finally, Fig. 16.36g, h demonstrates the molecular imaging capabilities of MSOT, which was employed in this case to detect upregulated epidermal growth factor (EGF) receptor in orthotopic pancreatic xenografts using a near-infrared EGF-conjugated CF-750 fluorescent probe (Hudson et al. 2014). The images clearly indicate the region where EGF-750 probe has accumulated with high spatial resolution. In comparison, fluorescence imaging of the mice also validated the accumulation of the EGF-750 probe at $6 \mathrm{~h}$ with specific accumulation in the lower left quadrant of the tumor (Fig. 16.36h). However, due to intense light scattering in deep tissues, the fluorescence measurements were only able to provide diffuse signal pattern with no depth resolution.

\subsubsection{Conclusions}

Owing to its hybrid nature, optoacoustics benefits from both the rich and versatile optical contrast and high (diffraction-limited) spatial resolution associated with the scattering-free nature of ultrasonic wave propagation. Much like other optical imaging modalities, optoacoustics uses safe nonionizing radiation at the visible and nearinfrared wavelengths. Thus, it provides an excellent platform for multiscale investigations using 


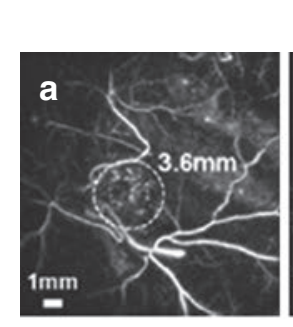

e
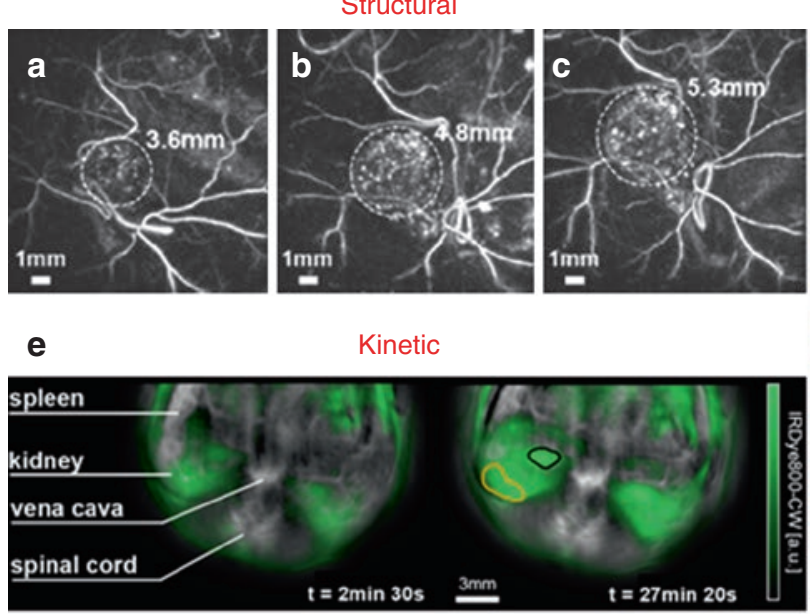

Kinetic

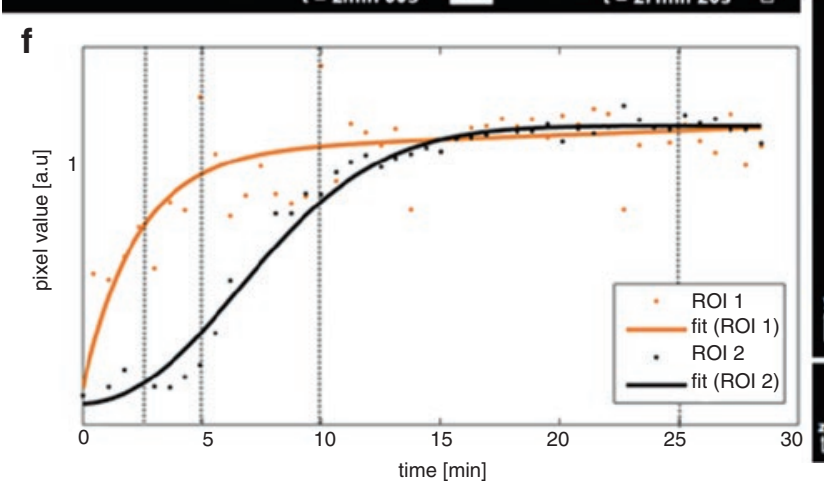

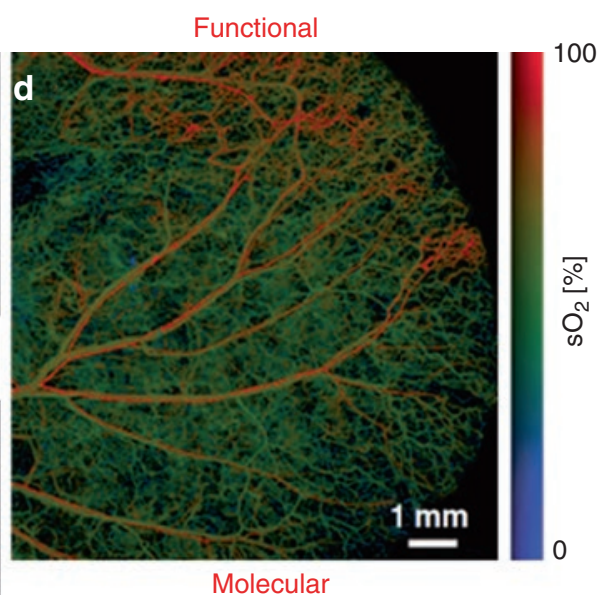

Molecular
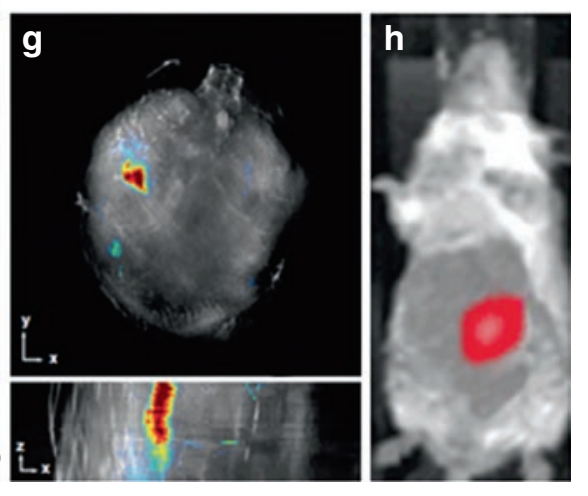

Fig. 16.36 Optoacoustic small animal imaging can provide multiple indications on in vivo structural, functional, kinetic, and molecular tissue profiles with a single modality. $(\mathbf{a}-\mathbf{c})$ show anatomical appearance of proliferating subcutaneous $293 \mathrm{~T}$ tumor and surrounding vasculature from day 7 to day 26 post inoculation (Laufer et al. 2012) (Reprinted with permission from SPIE). (d) Opticalresolution microscopy image representing distribution of blood oxygenation in a mouse ear ( $\mathrm{Hu}$ et al. 2011) (Reprinted with permission from the Optical Society of America). (e) Time series of cross-sectional whole-body mouse images visualizing the biodistribution of IRdye 800 near-infrared contrast agent in green overlaid on the anatomical image (Taruttis et al. 2012). (f) Temporal evolution of dye signal in the regions of interest highlighted in (e), orange showing a region in the renal cortex that displays early and steep signal pickup and black indicating a region in the renal pelvis where probe accumulation is

the same contrast, from microscopic observations at the single capillary and cell level to wholebody imaging of small animals and deep-tissue imaging of humans.

The optoacoustic effect relies on the absorption of light and is therefore ideally suited for delayed and has a smoother profile. Individual multispectral measurements acquired with a rate of approximately 2 per minute are shown as dots, while the solid line represents a fit to an analytical function (Reprinted with permission from the Public Library of Science). (g) Multispectral optoacoustic tomography (MSOT) detection of upregulated epidermal growth factor (EGF) receptor in orthotopic pancreatic xenografts using a targeted near-infrared EGF-conjugated CF-750 fluorescent probe (Hudson et al. 2014). Three-dimensional biodistribution of targeted EGF-750 probe is shown in color at $6 \mathrm{~h}$ postinjection. While MSOT can localize distribution of the probe with high resolution and all three dimensions, epifluorescence images (h) from the same animal have highly blurred low-resolution appearance with no depth-resolved information (Reprinted with permission from the American Association for Cancer Research)

vascular imaging since most of intrinsic contrast is obtained from high-hemoglobin-containing tissues like whole blood. This opens a multitude of potential applications in visualization of disease-related vascular changes, neovascularization, tumor microenvironment, and noninvasive 
functional diagnostics of blood perfusion and oxygenation. Yet, the capacity to image spectrally distinctive photo-absorbing agents with high spatiotemporal resolution at depths far beyond the diffusive limit of light opens unprecedented capabilities for functional and molecular imaging.

Extensive research is underway to address technical challenges associated with the intriguing and highly promising combination of light and sound. Main limitations are currently associated with the lack of reliable and affordable laser and ultrasound detection technology that can optimally address the unique needs of optoacoustics, such as high per-pulse laser energy or repetition rate, ultrawideband detection, high detection sensitivity, and miniaturization. Multiple frontiers are also open in the algorithmic and inverse theory areas, trying to address challenges related to artifact removal, image quantification, reconstruction strategies in the presence of acoustically mismatched areas, real-time operation, and multispectral data processing.

All in all, optoacoustic imaging attracts growing interest from the biological and medical research communities, as manifested by the everincreasing number of publications encompassing great variety of new applications that exploit the unique advantages offered by the technology. It is therefore expected to find broad applications in biological research and clinical practice in multiple fields, including cancer research, functional brain imaging, cardiovascular imaging, gastroenterology, ophthalmology, drug development, and treatment monitoring.

\subsection{Optical Probes}

Kai Licha

\subsubsection{Introduction: Optical Probes for Animal Imaging}

The application of optical imaging technologies for drug discovery research and the development of novel preclinical animal models have expanded with tremendous vigor in the past few years. The fact that fluorescent dyes can be detected at low concentrations and nonionizing and harmless radiation is applied with rather low technical effort makes optical techniques attractive for routine use in the animal imaging laboratory. Novel imaging probes and contrast agents have been designed in a broad variety addressing the various requirements given by the disease problem at the preclinical animal imaging stage. Moreover, the industry has identified this field as a market from the side both the imaging equipment and the fluorescent probes applied as readily injectable contrast agents or reactive labels for bioconjugation chemistry.

Imaging devices with powerful and versatile capabilities are now commercially available, addressing different needs of the customer, such as multicolor imaging, time-resolved imaging, planar or tomographic geometry, signal quantification, spectral unmixing for background correction, or zooming within macroscopic scale. A closer look into these topics is provided elsewhere in this book.

The design of fluorescent imaging probes for in vivo imaging applications has likewise emerged and is reflected by an increasing number of commercialized probes and labels. The underlying chemistries have been optimized for their capability to monitor disease-specific anatomic, physiological, and molecular parameters by way of directly applicable imaging probes or by specific reactive labels, which can be used by the customer to fluorescently label his own drug candidate for further studies or derivatize important biological ligands to investigate the basics of a given targeting approach.

The main objective of this contribution is to provide an overview on the different choices available when setting up an imaging protocol using optical probes. Hereby, the review sets a focus on practical aspects of the application of optical probes for animal imaging and drug discovery research. The state of the art of imaging probes is first discussed covering nontargeted and vascular agents, approaches to generate targeted agents via reactive fluorophores, the field of enzymatically activatable probes, and imaging agents 


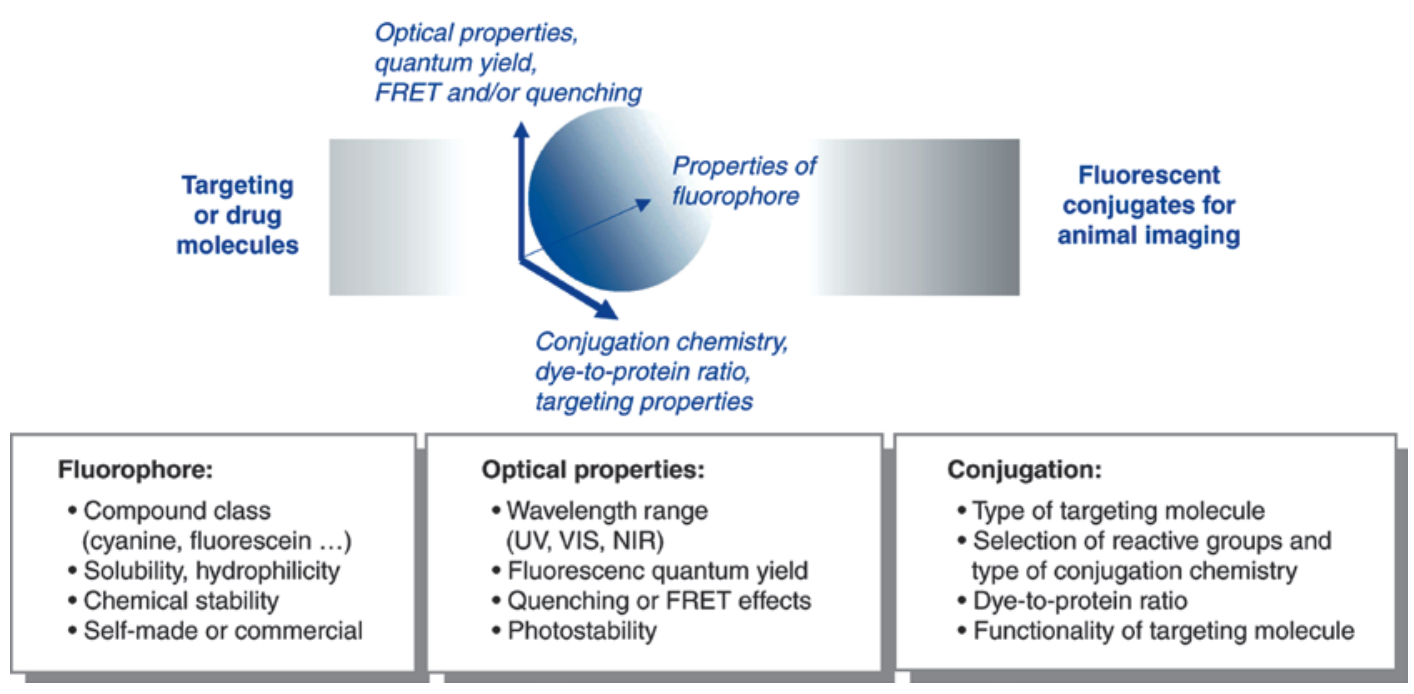

Fig. 16.37 Basic idea and general parameters for the generation of optical probes

based on particles. To facilitate the researcher's choice, a selection of relevant parameters is discussed in the subsequent chapter, discussing appropriate examples out of the literature. At this point, it is mentioned that examples include published work and refer to selected commercial compounds and companies as well. The intention of this review is neither to provide a comprehensive dataset on these probes nor to accentuate particular commercial suppliers. Figure 16.37 summarizes the general parameters that contribute to the design of optical imaging probes.

\subsubsection{State-of-the-Art Technologies}

\subsubsection{Basics on Fluorescent Dyes}

An efficient fluorescence imaging probe should employ a combination of different properties, which are (1) a high fluorescence quantum yield in the desired wavelength spectrum, (2) sufficient biological stability and photostability to permit unimpaired image acquisition, and (3) reasonable solubility in (most) aqueous environment to enable bioconjugation and labeling reactions without substantial triggering of aggregation and/ or precipitation. Among the many possible organic fluorophore classes, the polymethine dyes (e.g., cyanines, hemicyanines, benzopyrylium dyes), xanthene dyes (rhodamines and fluoresceins), and also some oxazoles and thiazoles (e.g., methylene blue, Nile blue) are basically the ones that are widely established and have been contributing to the majority of scientific results (Licha and Resch-Genger 2011, 2014). Figure 16.38 illustrates typical absorption and fluorescence spectra of these types of fluorophores, thereby showing the wavelength range within imaging is possible. While xanthene dyes do not reach fluorescence emission far beyond $700 \mathrm{~nm}$, they exhibit extremely high fluorescence quantum yields often close to $100 \%$. In turn, cyanine dyes are capable to cover the entire spectrum from VIS to NIR (>900 nm) with high extinction coefficients (up to $250,000 \mathrm{~L} / \mathrm{mol} \mathrm{cm}$ ), but rather moderate fluorescence quantum yields (up to $30 \%$ ). Other interesting but less frequently applied organic dyes include squaraines (Luo et al. 2011b) and BODIPYs (Boens et al. 2012). Besides organic fluorophores, inorganic semiconductor nanoparticles have emerged as alternative fluorescent reporters (see Chap. 21.2.5).

As further detailed in the subsequent chapters, the most relevant applications for these dyes as imaging tools are the direct use as injectable contrast agents in a nontargeted or passively distributing format, the use as reactive fluorophore 


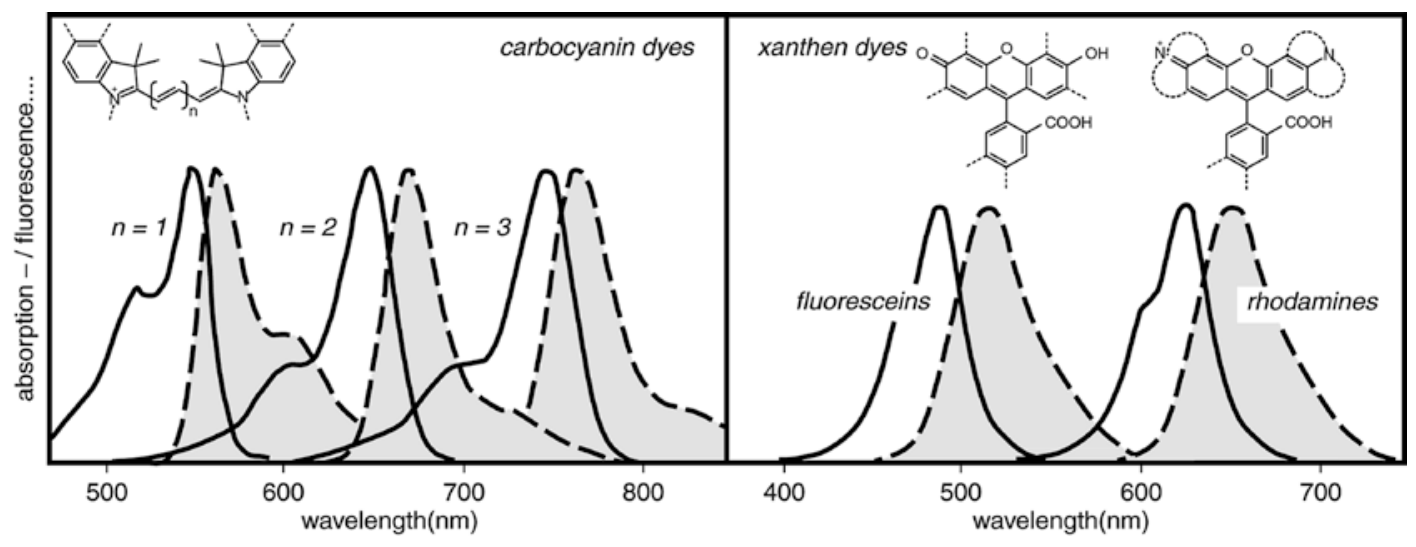

Fig. 16.38 Typical absorption and fluorescence spectra of cyanine dye and xanthenes dyes shown as idealized illustration

labels to create targeted conjugates of biological or synthetic origin, and the use as quenched components or non-fluorescent precursors as activatable imaging probes (also named "turn-on" probes) to monitor different kinds of enzyme activity. Furthermore, pH-responsive fluorophores enabling the sensing of $\mathrm{pH}$ in tissues have been approached as additional parameter in tumor models.

\subsubsection{Nontargeted and Vascular Dyes as Contrast Agents}

A variety of fluorescent dyes for passive targeting and vascular circulation have been applied as fluorescent imaging probes. These probes, most of them carbocyanine dyes, represent the basic chemicals for the design of labels and probes. Using these dyes as imaging agents by themselves, they act by their physicochemical and structural properties since they do not employ structures designed for a specific molecular interaction. However, the suitability for animal studies, e.g., the characterization of experimental tumor models, has been demonstrated in various publications.

The near-infrared dye indocyanine green (ICG, absorption $~ 780-800 \mathrm{~nm}$ ) was approved in the 1960s as a diagnostic drug, was rediscovered in the 1990s as an imaging agent, and is today, together with fluorescein, frequently applied for fluorescence angiography in ophthalmology (Richards et al. 1998), as well as increasingly used for fluorescence methods in surgery (Alander et al. 2012). Similarly, novel cyanine dye derivatives have been created as passively targeted contrast agents of simple synthetic availability. Examples are the dye SIDAG, which leads to enhanced uptake in a variety of tumor models and permits differentiation of the degree of angiogenesis (Wall et al. 2008). Furthermore, the compound was shown to enable fluorescence imaging of inflammation in a model of rheumatoid arthritis (Fischer et al. 2006). It therefore provides a powerful tool for the validation of preclinical models, imaging equipment, and therapeutic studies (TryX750, mivenion). With the IRDyes (LI-COR Biosciences) or Cy5.5 (GE Healthcare Life Sciences), other examples of nontargeted or control dyes with published results for animal imaging and human applications, such as lymphatic mapping or studies on T-cell migration, accompany ICG (Foster et al. 2008; Rasmussen et al. 2009). Another class that has recently attracted attention is the benzopyrylium-based polymethine dyes (Dyomics) (Pauli et al. 2009b). In Fig. 16.39, a panel of selected chromophores is depicted. Beyond ICG, which by itself is not directly applicable to synthesize reactive derivatives for biolabeling due to the absence of chemical functionalities, the compounds discussed here represent basic structures to create in few further steps reactive labels and subsequently any desired bioconjugate. 


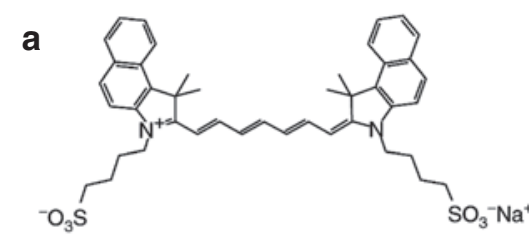

C

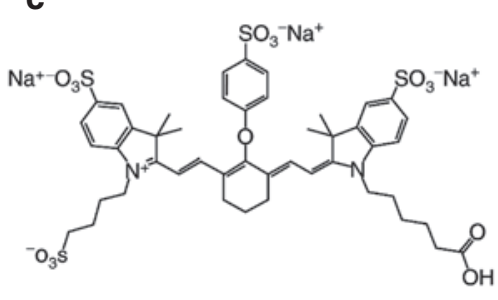

Fig. 16.39 Selected near-infrared fluorescent dyes, which have been used as nontargeted probes for in vivo fluorescence imaging: (a) indocyanine green, (b) SIDAG

Cyanine dye conjugates with substantially increased molecular weight have been designed to enable the visualization of vascular structures and to study effects on the vascularity. For this purpose, the dyes were covalently conjugated to macromolecular carriers, such as albumin, dextran, or other biopolymers (Klohs et al. 2009) or non-covalently entrapped into dendritic architectures used as passive drug delivery systems (Quadir et al. 2008). These conjugates have been useful for imaging tumors but also to visualize and characterize tissue architecture in vivo on a macro- to microscopic imaging level. Commercialized are, e.g., streptavidin, ovalbumin, protein $\mathrm{A}$, and dextran conjugates with a variety of Alex Fluor ${ }^{\circledR}$ labels, marketed under the brand SAIVI ${ }^{\mathrm{TM}}$ (Invitrogen) as imaging probes and secondary detection materials. Other available probes are the Angio Sense $\AA$ probes (Perkin Elmer) based on large, long-circulating macromolecules at $250 \mathrm{kD}$ (type of macromolecular carrier not published).

\subsubsection{Reactive Dyes and Labeled Targeting Molecules}

This chapter addresses the increasing demand for fluorescently labeled drug candidates based on molecules of synthetic (small organic molecules, peptides, oligonucleotides) or biotechnological

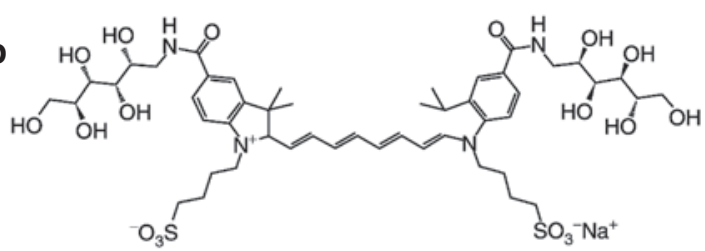

e

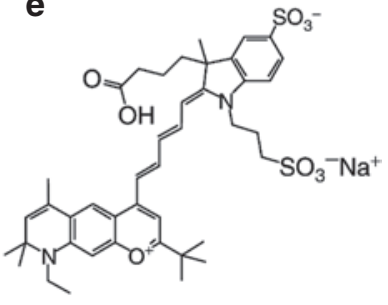

(Wall et al. 2008), (c) IRDye® 800CW (LI-COR, [394]), (d) Cy5.5® carboxylic acid (GE Healthcare) and (e) DY-750 (Dyomics)

origin, such as engineered proteins, antibodies, and novel protein formats. In order to get an insight into the targeting capabilities of these drugs in vivo, optical imaging is establishing as a fast and easy-to-apply technology in the animal imaging laboratory. As further outlined in Chap. 21.3., fluorescence labeling offers the opportunity to study biological and physicochemical properties of a desired candidate along the entire path from synthesis, in vitro and cellular level to the in vivo application in higher organisms.

Generally, optical imaging probes in the format of bioconjugates range from protein conjugates, antibody conjugates, structures based on oligonucleotides to receptor-avid peptide conjugates. Furthermore, a large panel of smallmolecule substrates and ligands, such as folate, cyanocobalamin, glucose, bisphosphonates, as well as rationally designed receptor ligands, has been conjugated with fluorophores for in vivo studies.

This work has ultimately led to a large variety of targeted imaging agents published in the past few years. A selection of different reactive dye labels and resulting conjugates is depicted in Fig. 16.40. Being not the intention of this chapter to discuss these versatile approaches in detail, the reader is referred to respective reviewing publications (Licha and and Resch-Genger 2014; 

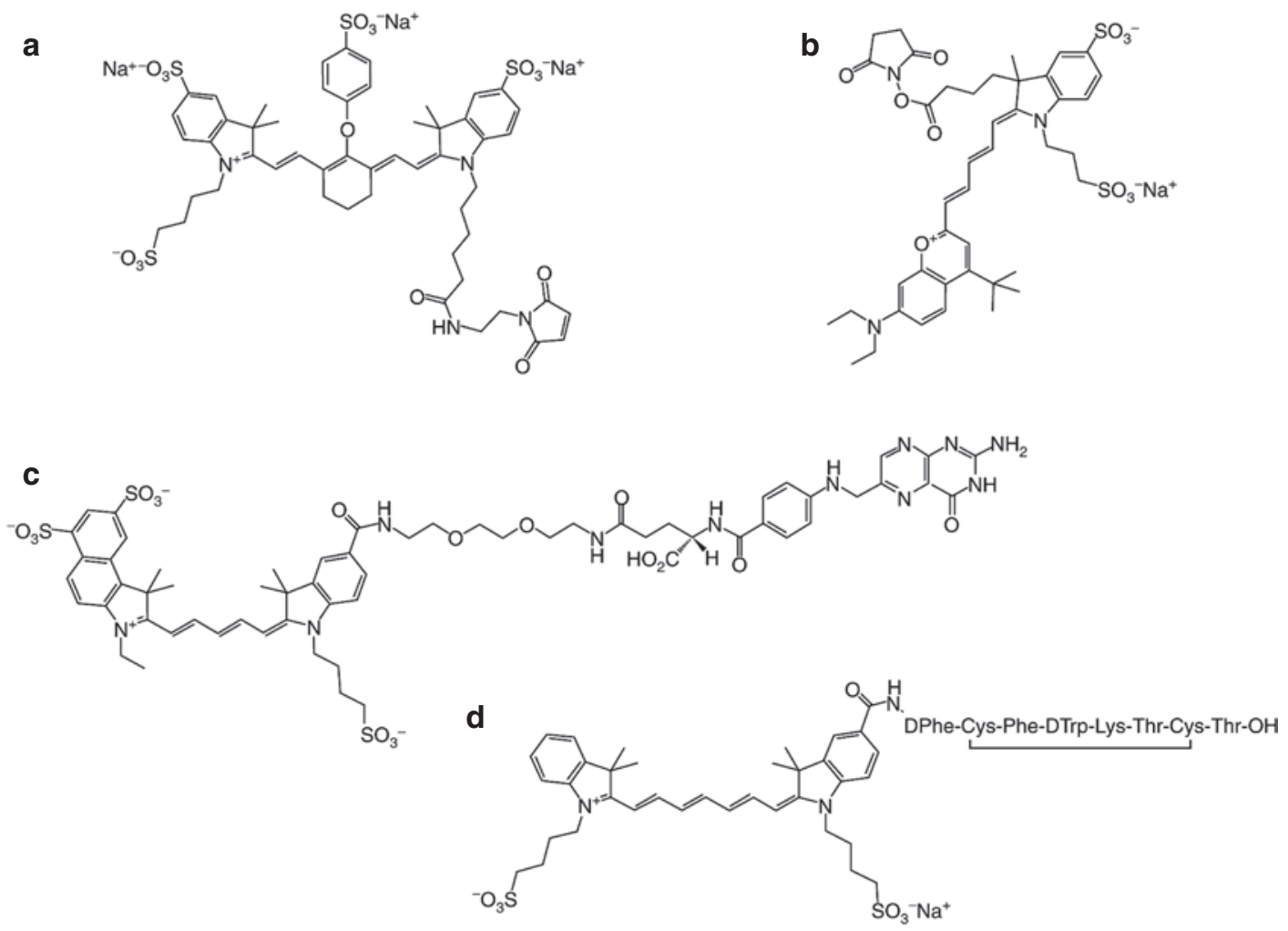

Fig. 16.40 Selected dyes in a reactive format for biolabeling and published bioconjugates: (a) IRDye® $800 \mathrm{CW}$ Maleimide (LI-COR), (b) DY-781 NHS-ester (Dyomics),

Umezawa et al. 2014). A number of conjugates have been made commercially available addressing fundamental pathways in cellular uptake, angiogenesis, and tumor growth, for instance, conjugates with EGF (LI-COR), transferrin, annexin $\mathrm{V}$, bombesin, folate, glucose, or certain bisphosphonates with bone affinity (Perkin Elmer).

Oligonucleotides and small peptides, as opposed to, e.g., targeting molecules of biotechnological origin, are accessible via solid-phase synthesis with the fluorophore conjugation capable of being implemented into the synthetic protocol. Most of the dyes used have shown to be stable enough with respect to the chemical conditions required for peptide cleavage from the resin and other interventions (e.g., forming disulfide bridges via oxidation). Examples for the synthesis of low-molecular-weight peptide conjugates comprising different cyanine or rhodamine dyes (c) Cy5.5-folate conjugate (Moon et al. 2003), and (d) indotricarbocyanineoctreotate (Becker et al. 2001)

have been published (Becker et al. 2001; Mier et al. 2002). For the $3^{\prime}$ and $5^{\prime}$ labeling of oligonucleotides, conjugation chemistry can be applied similarly. A broad set of labels and quenchers covering the entire VIS to NIR is, for instance, offered with the ATTO dyes (ATTO-TEC).

\subsubsection{Activatable or "Turn-On" Probes}

Weissleder and coworkers have introduced an optical imaging approach, which is of fundamental difference to the approaches described above and utilizes the unique opportunity of modulating optical signals through intramolecular fluorescence quenching effects (Hilderbrand and Weissleder 2010; Weissleder et al. 1999). These probes are "activatable" polymeric conjugates based on a poly-L-lysine/polyethylene glycol graft polymer, which are "switched on" when 
single fluorochrome fragments are cleaved from the fluorescence-quenched polymeric structure by tumor-specific proteolytic enzymes, thus leading to signal amplification of up to 200 -fold. In various preclinical disease models, the agents have demonstrated the imaging enzymatic activity and report the in vivo efficacy of enzymeinhibiting drugs (Bremer et al. 2001b). The underlying imaging probes are based on cleavable graft polymers of high molecular weight and are commercialized by VisEn Medical Inc. Under different trade names (ProSense ${ }^{\circledR}$, MMPSense $\AA$ ), the compounds are cleaved and activated by enzymes such as cathepsins and matrix metalloproteinases at spectral ranges of 680 and $750 \mathrm{~nm}$, respectively.

Rationally designed conjugates of defined chemical structures, activatable photosensitizers for photodynamic therapy (Stefflova et al. 2007), as well as constructs sensitive to cleavage by other enzymes, such as thrombin and caspases (Barnett et al. 2009), have expanded this versatile technology. More recently, the capability to turn on fluorescence by an enzymatic conversion has been implemented into the fluorophore structure itself, thereby circumventing the need to employ a certain number of molecules to generate quenching (Kisin-Finfer et al. 2014). The advantage of such structures is the extremely low background in the switched-off state, simply because this precursor does not contain generate background due to the absence of a fully established chromophore.

\subsubsection{Probes for Sensing of $\mathrm{pH}$ and Reactive Oxygen Species}

The establishment and characterization of tumor models might benefit from knowledge about certain physiological parameters, such as tumor $\mathrm{pH}$ (extracellular or intracellular) and the level of oxidative stress (reactive oxygen species), both of which being valuable in combination with molecular conditions (Licha and Resch-Genger 2014).

Examples for $\mathrm{pH}$-responsive xanthene dyes are BCECF (2',7'-bis-(2-carboxyethyl)-5-(and-6-)carboxyfluorescein) and BCPCF (2',7'-bis(2-carboxypropyl)-5-(and-6)-carboxyfluorescein) (see also the Oregon Green series from Life Technologies). Beyond fluoresceins and rhodamines, similarly, benzoxanthenes have been synthesized in many versions combining the advantages of fluoresceins and rhodamines, such as the SNARF and SNAFL dyes (seminaphthorhodafluor and semi-naphthofluorescein derivatives, respectively). Typically, these dyes do not show significant $\mathrm{pH}$-dependent changes in emission. Accordingly, these probes are termed dual or ratiometric excitation probes for $\mathrm{pH}$, requiring excitation at two wavelengths.

More relevant for imaging purposes are NIR cyanine dyes which exhibit responsiveness to $\mathrm{pH}$ changes by omitting one substituent in the indole end groups. Recent examples include the commercially available CypHer5E dye label (Mathejczyk et al. 2012) and other similarly constructed NIR-emissive pH-responsive cyanine dyes (Lee et al. 2011), which have been applied for in vivo imaging thereby showing advantages in tumor detection and background suppression.

The term reactive oxygen species (ROS) encompasses oxygen radicals and peroxides such as $\bullet \mathrm{OH}^{-}, \cdot \mathrm{O}_{2}^{-}$, and $\bullet \mathrm{OOH}$ which are important indicators for the oxidative state of cells, in particular as a result of certain therapeutic intervention. Thus, fluorescent sensors and stimuli-responsive fluorophores have emerged as imaging tools. An example are so-called hydrocyanines (Selvam et al. 2011) which change from a reduced, noncolored status to the chromophore structure upon oxidation in living systems.

\subsubsection{Fluorescent Particles as Imaging Agents}

Fluorescent imaging agents based on particles have shown great utility, especially for applications on cellular staining (in vitro) and vascular imaging (in vivo). In principle, there are two different groups of nanoparticle probes. On the one hand, nanosized materials based on organic and/or inorganic chemistry have been equipped with organic fluorophores, by either incorporating the dye molecules into the nanoshells or attaching it to surface functionalities (Azar and Intes 2008). Interesting nanomaterials, including semiconductor nanoparticles 
(quantum dots), gold nanoparticles, and carbonbased particles (nanotubes), have emerged as powerful imaging tools with brilliant optical properties (Holzinger et al. 2014). Probably in a most advanced state of utility are quantum dots which consist of atoms such as $\mathrm{Cd}, \mathrm{Se}, \mathrm{Te}, \mathrm{S}$, and $\mathrm{Zn}$. The fluorescence emission range depends on the diameter of the particles, which can be synthesized in a very controlled fashion with respect to size and surface modifications (Fig. 16.41). Surfaces employing stabilizing polymers as well as targeting molecules, such as antibodies and peptides, have expanded their applications for in vivo animal imaging (Bentolila et al. 2009).

Nanoparticles are particularly interesting for multimodality imaging purposes due to their modular composition. Magnetic cores for MRI imaging can be functionalized with additional fluorophores to allow fluorescent tracking and vice versa; fluorescent cores have been combined with paramagnetic complexes (Azar and Intes 2008).

\subsubsection{The Choice of Parameters}

\subsubsection{Optical Properties}

Generally, the choice of the spectral range of absorption and fluorescence of the dye determines whether it is detectable on tissue surfaces
(UV-vis dyes, 400-700 nm) or from deeperlocated tissue areas (NIR dyes, $>700 \mathrm{~nm}$ ). A prerequisite for sensitive detection of a contrast-enhancing dye is a high extinction coefficient at the desired absorption wavelength. If fluorescence is recorded within the UV/VIS spectral region, both autofluorescence and the administered contrast agent will contribute to the observed signal, while in the NIR spectral region, tissue autofluorescence is negligible due to the absence of endogenous NIR fluorophores. In the latter case, the detected signal nearly exclusively reveals the distribution of the optical imaging probe.

The broad availability of fluorophore labels allows the selection of probes exactly matching the technical parameters of a given analytical instrument. Under practical circumstances is the application of one particular fluorophore for a research program starting at the in vitro level with cell microscopy, FACS, and other assays, then reaching in vivo imaging studies in animals, often not possible. Analytical instruments are usually not equipped with excitation and detection components permitting detection with sufficient sensitivity over the entire spectral range. Therefore, a work-around strategy often involves the choice of an appropriate series of fluorophores that differ only in minor extent in their

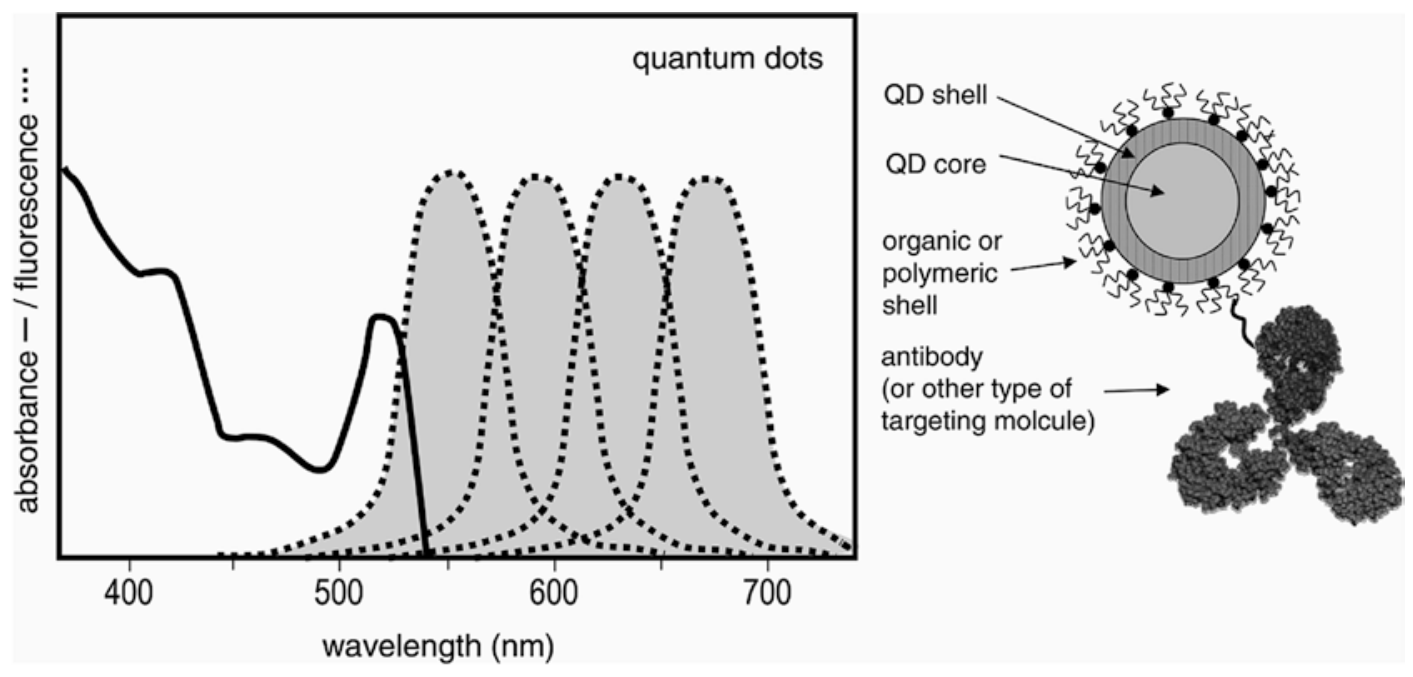

Fig. 16.41 Typical absorption and fluorescence spectra of quantum dots shown as idealized illustration; principal design of targeted quantum dot conjugates 
chemical structures. This strategy minimizes unexpected effects on the resulting targeting conjugate when changing the fluorophore backbone. Here, cyanine dyes with their carbo-, dicarbo-, and tricarbocyanine homology are well suited. Examples of dyes offered as series are the CyDye $^{\mathrm{TM}}$ series (GE Healthcare Life Sciences), which have been one of the first comprehensive product lines for fluorescence labeling (Cy3, Cy5, Cy5.5, Cy7).

The Alexa Fluor ${ }^{\circledR}$ Dye series (Invitrogen/ Molecular Probes) have expanded the available spectral emission range from approximately 450 to $800 \mathrm{~nm}$ including derivatives (cyanine and xanthene) of improved photostability and fluorescence quantum yields. Alexa Fluor 488, Alexa Fluor 546, Alexa Fluor 555, and Alexa Fluor 750 are examples for products (consistently named by their excitation maximum). Similarly, highly hydrophilic Cy5.5-like pentamethine dyes have been reported in comparison to the known Cy5.5 label (LI-COR and (Pauli et al. 2013)). Another group is based on a different chromophore type, the borondipyrromethenes, known as BODIPY dyes (Invitrogen) (Boens et al. 2012). For a representative cyanine dye series, the typical color appearance of solutions (approximately $0.1 \mathrm{mM}$ ) in water is depicted in Fig. 16.42, which might facilitate the identification of the correct probe in case of some disorientation caused by too many flasks at the same time on the bench.

Generally, reactive dyes are offered in different version of chemoselectivity (see Chap. 21.3.3) so that the most reasonable labeling chemistry can be applied. The obtained amount of conjugate has to match the extent of the planned animal imaging study. Typically, fluorescent conjugates have been applied at doses in the range of $0.1-1 \mu \mathrm{mol} / \mathrm{kg}$ (i.v.), which corresponds to approximately $0.1-1 \mathrm{mg} / \mathrm{kg}$ amount of fluorophore (calculation based on the molecular weight of the fluorophore). Under technical aspects, the dose should be chosen such that a reasonable enhancement of the overall signal intensity in the animal directly after administration is detected (e.g., at least tenfold increase compared to background signal before injection).

\subsubsection{Pathophysiological Paradigm}

Viewing the applications of optical imaging probes from the angle of their pathophysiological function, it appears as common sense to group the probes into nontargeted or vascular agents, targeted conjugates, and activatable probes. As detailed in the preceding chapter
Fig. 16.42 Photograph of cyanine dye solutions: (a) indocarbocyanine with abs/fluor $550 / 580 \mathrm{~nm}$,

(b) indodicarbocyanine $650 / 680 \mathrm{~nm}$, and (c) indotricarbocyanine $750 / 780 \mathrm{~nm}$.

Concentration of solutions approximately $0.1 \mathrm{mM}$ in water

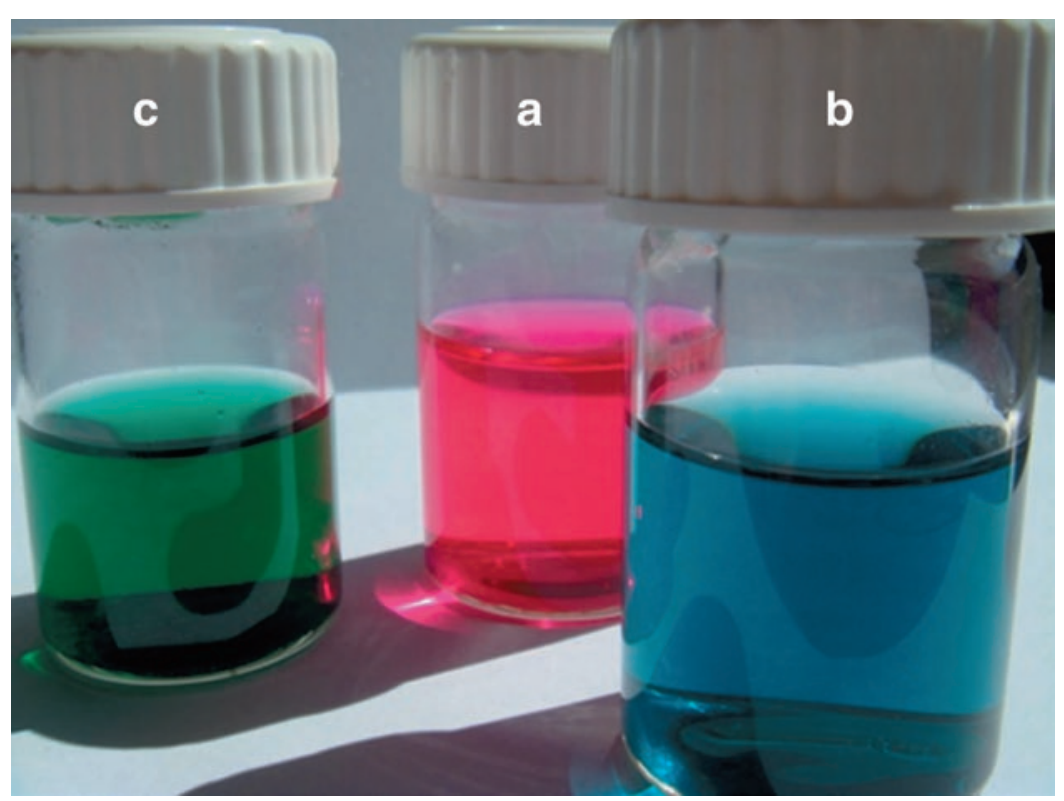


on state-of-the-art technologies, a host of compounds have been designed and studied for each of these three fundamental strategies. It depends on the rationale of the respective scientific problem to be solved by the application of in vivo optical imaging. Vascular or nontargeted fluorophores, as well as circulating fluorescent particles might be used first when a general imaging protocol is to be established, for the characterization of novel disease models (growth characteristics, vascularity, tumor sizes, formation of metastases, etc.). Other interesting aspects refer to the dose finding, detection limits, and image acquisition parameters. Targeted conjugates permit the imaging of molecular information, such as specific cellular expression profile. Fluorescently labeled drug candidates can be monitored with respect to their in vivo uptake into the target tissue and their capability to bind the molecular target. The underlying rationale is often to study the pharmacokinetic behavior of the probe, which has to fulfill certain properties with respect to their adsorption, distribution, metabolization, and excretion (ADME) for the living organism. Optical imaging can provide a fast and easy access to semiquantitative ADME data; however, a true quantification of drug amounts and the fate of metabolites in the organs are technically difficult. Here, radioactive techniques are a method of choice. Activatable probes provide insight into the third level of detecting protein function, such as the activity of proteolytic enzymes. Here, the injected imaging probe is silent until activated by the corresponding enzyme. Accordingly, a concentration ratio between diseased tissue and normal areas is not necessarily a requirement as long as the circulating fraction of the probe remains undetectable. This technology has shown to be applicable for the imaging of enzyme inhibitors in drug screening protocols (Fig. 16.43).

Probably, the combined exploitation of these mechanisms would allow that the deepest insight into the physiology and molecular biology of disease formation and progression be gained. At this point, it is worth to emphasize that a particular opportunity of fluorescence detection is the principal capability of multicolor imaging given that the imaging instrumentation permits multiple excitation and/or detection wavelengths. To give an example, the tumor vascularity would be detectable by a long-circulating vascular probe (e.g., $680 \mathrm{~nm}$ excitation), while the analysis of a certain receptor expression could be accomplished by a target-specific conjugate working at 750 or $800 \mathrm{~nm}$.
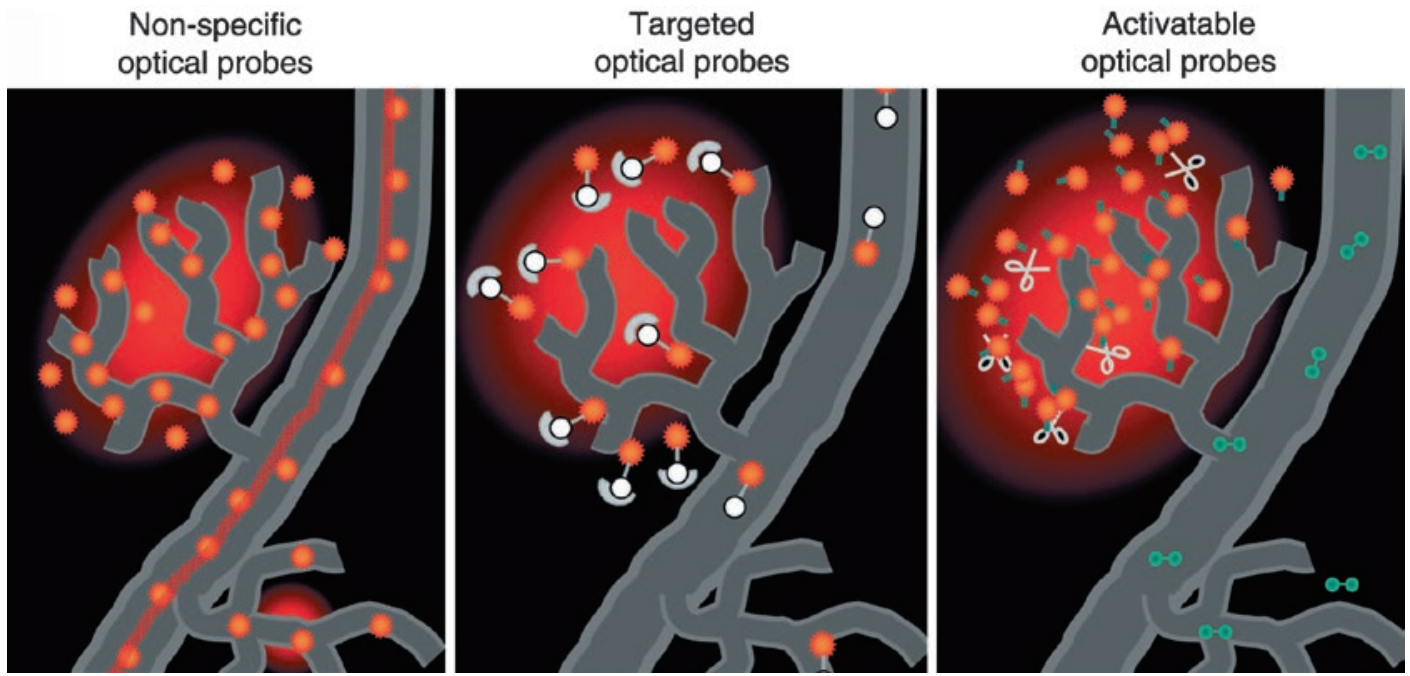

Fig. 16.43 Illustration of the different mechanisms of contrast generation by fluorescent imaging probes. In principle, the target-to-background ratios of signals and resulting imaging contrasts increase from nonspecific optical probes to targeted conjugates and activatable probes (Modified with permission from Sonja Vollmer) 


\subsubsection{Type of Labeling Chemistry}

For the labeling of proteins, antibodies, or other macromolecules, the type of labeling reaction has to be considered. Generally, different residues in the macromolecule are available for covalent attachment to reactive fluorophores. The most relevant reaction sites are amino groups (lysines), thiol groups (cysteine), hydroxyl groups (threonine, serine, and glycans), and aldehydes (generated from oxidative cleavage of glycosylated structures). The principle pathways of covalent labeling of amino and thiol groups (provided by the amino acids, lysine, and cysteine, respectively) are illustrated in Fig. 16.44. Labeling of lysines is most established by using $N$-hydroxysuccinimidyl esters of dyes, easily accessible from carboxylic acid groups. Another approach is the utilization of isothiocyanate groups (most prominent is fluorescein isothiocyanate, FITC). Labeling of cysteines occurs most conveniently with maleimides or with pyridinium disulfides. A combined strategy involves the thiolation reagent iminothiolane (Traut's reagent), which enables the conversion of an amine group into a linker with free thiol group for subsequent maleimide labeling (Hermanson 1996).

Labeling usually occurs with high chemoselectivity, allowing that cysteines or lysines be targeted independently. In native proteins, labeling occurs statistically yielding an average dye-toprotein ratio with randomly distributed fluorophores due to the randomly located cysteines or lysines in the protein. In this respect, reactions at sensitive binding sites or amino acids involved in the molecular function can hamper the affinity of the biomolecule. Therefore, the labeling approach should consider the protein sequence in order to select the appropriate labeling reaction.

A complementary approach can be followed by employing structures of glycosylation by reducing sugar moieties in a way that aldehyde functionalities are generated. These aldehyde groups react readily with amino groups (forming imines) or hydrazine groups (forming hydrazones), both of which are acid cleavable but can be chemically stabilized by reduction with sodium cyanoborohydride (Hermanson 1996).
Thiols are usually accessible by reductive breaking of disulfide bonds in the protein. However, since these bonds are crucial for protein folding and stability, denaturation and loss of target binding capability can occur. A structurally more defined approach can be followed by using engineered antibodies with additional cysteine residues, which are not involved into protein structure and are free to be used for covalent labeling (Lee et al. 2008).

As outlined in Chap. 21.2, a host of fluorophores are available for the different bioconjugation chemistries. In a typical protein-labeling protocol, a buffered solution ( $\mathrm{pH} 7.5-8)$ of the protein $(1-10 \mathrm{mg} / \mathrm{mL})$ is incubated with a molar excess of reactive dye (typically 10-50 moles of dye per mole biomolecule) for a few minutes to several hours depending on dye reactivity and desired labeling ratio. Purification and separation from unbound dye can be achieved by separation methods such as dialysis, ultrafiltration, or size exclusion chromatography. In order to determine the quality of the resulting conjugate, suitable analytical methods are needed subsequently.

\subsubsection{Conclusion and Outlook}

Optical imaging has an established role in preclinical research and animal imaging. The application of imaging techniques for the detection for fluorescence signals in living animals has emerged to a powerful routine level and utilizes a versatile set of commercially available imaging equipment. The detection of fluorophores as exogenously applied contrast agents can be accomplished with convenient handling and without a substantial effort regarding technical installation, safety issues, and training. A broad variety of fluorescent probes have been created and validated for their ability to facilitate the setup of imaging protocols, to support the characterization of disease models, and to accompany the drug discovery process from drug design, in vitro characterization to the in vivo proof of concept. Both the selection and application of commercially available products optimized for a 

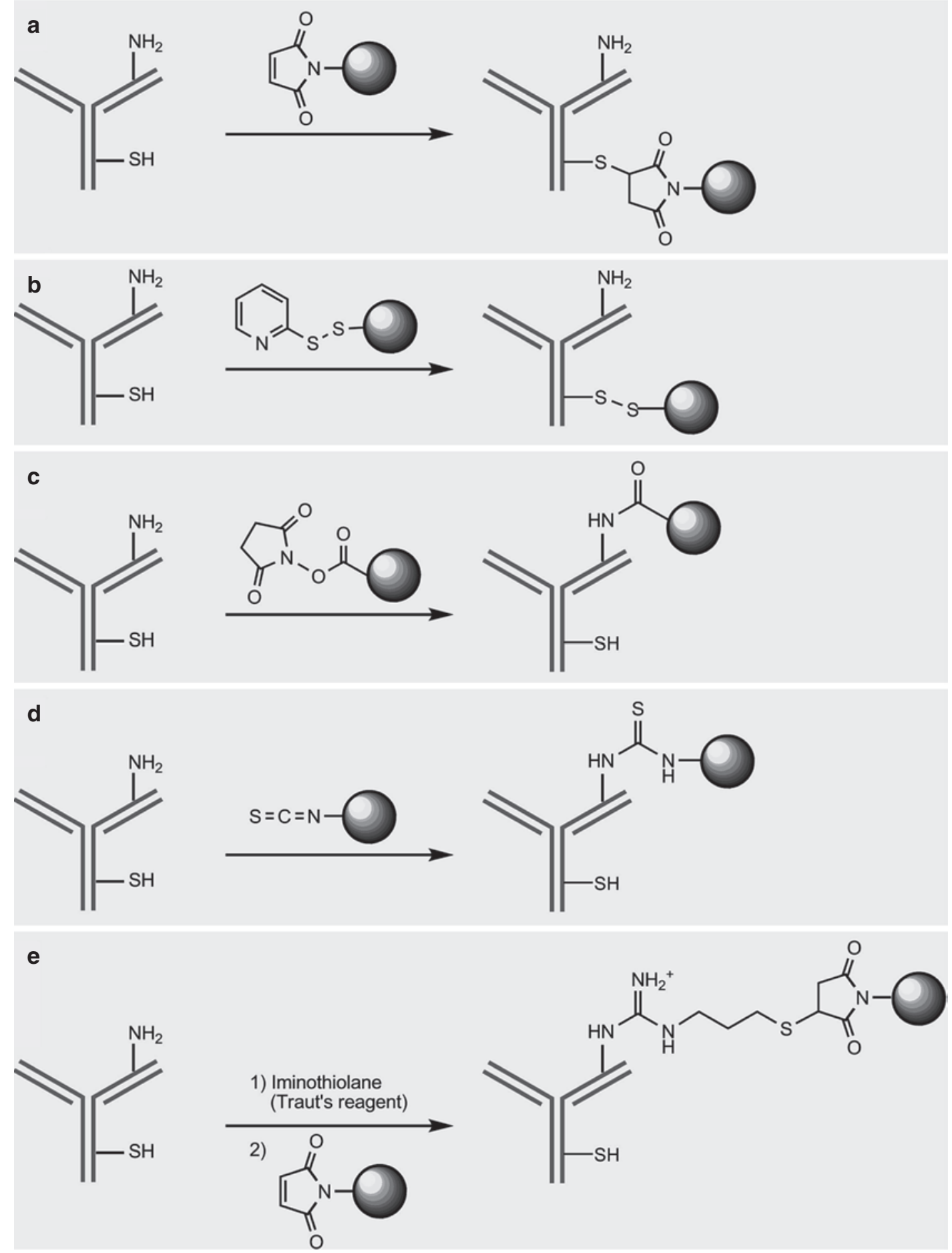

AU22 Fig. 16.44 Illustration of synthetic pathways for selective labeling of antibodies or other proteins; the fluorophore is simplified as bulb. Depicted are reactions of (a) maleimide dye with cysteine, (b) pyridinium disulfide dye with cysteine, (c) NHS-ester dye with lysine, (d) isothiocyanate dye with lysine, and (d) modification of lysine with Traut's reagent to give thiol, followed by reaction with maleimide dye 
specific function as well as the implementation of self-induced synthetic chemistry adapting to the requirements in a given drug discovery project will play a central role in the optical imaging laboratory.

\subsubsection{Laboratory Protocols}

\subsubsection{Labeling of Antibody with Dye NHS-Ester}

Generally, antibodies should be used in a buffer free of amines. For instance, phosphate, acetate, or borate buffers are recommended, whereas TRIS and HEPES buffers are less suited. Incubation is performed with large excess of dye, followed by removal of excess nonreacted dye by using gel chromatography, dialysis, or ultracentrifugation. The latter two methods bring the dye conjugate into contact with material surfaces for which precipitation and loss of reaction yield are sometimes observed especially when using less hydrophilic dyes with tendency to aggregate. The following steps are recommended:

- Dissolve antibody or protein to be labeled in reaction buffer at concentration of $1-10 \mathrm{mg} /$ $\mathrm{mL}$ depending on solubility (buffers, e.g., $100 \mathrm{mM}$ sodium phosphate $\mathrm{pH}$ 7.4-8.0).

- Dissolve the reactive NHS-ester dye in a small amount of DMSO or DMF (concentration approximately $10 \mathrm{mM}$ or $1 \mathrm{mg} / 100 \mu \mathrm{L}$ ). In case of high water solubility, the dye can be dissolved in buffer but should then be used immediately due to starting hydrolysis of the NHS-ester group.

- Add the stock solution of dye to the antibody/ protein solution to achieve the desired molar excess. For instance, 15-fold molar excess is generated by adding $10 \mu \mathrm{L}$ of dye solution (concentration $10 \mathrm{mM}$ ) to $1 \mathrm{~mL}$ of antibody solution (concentration $1 \mathrm{mg} / \mathrm{mL}$, IgG molecular weight $150 \mathrm{kD}$ ).

- Incubate for 3-24 h under gentle shaking at room temperature and under protection from light.

- Remove excess dye by gel filtration, e.g., by using desalting columns. Best results are obtained when not loading the columns at the maximal possible volume, such as using a NAP10 column for not more than $0.5 \mathrm{~mL}$.

\subsubsection{Labeling of Antibody with Dye Maleimide}

This example describes the labeling of a cysteine after reductive generation of free thiol. A mild reducing agent is, e.g., TCEP (triscarboxyethylphopshine), which reaches surface disulfides while leaving deeper bridges in the protein backbone intact. Usually, maleimide dyes can be used with less molar excess compared to NHS esters. The following steps are recommended:

- Dissolve antibody or protein to be labeled in reaction buffer at concentration of $1-10 \mathrm{mg} /$ $\mathrm{mL}$ (buffers, e.g., $100 \mathrm{mM}$ sodium phosphate pH 6.5-7.0).

- Dissolve TCEP in reaction buffer (concentration approximately $10 \mathrm{mM}$ ).

- Add the TCEP solution to the antibody/protein solution to achieve a molar excess of 5-10 molecules TCEP per disulfide to be reduced.

- Incubate for $2 \mathrm{~h}$ under gentle shaking at room temperature.

- Add the stock solution of dye to the freshly reduced antibody/protein solution to achieve the desired molar excess (five- to tenfold).

- Incubate for another 2-4 h under gentle shaking at room temperature.

- Remove excess dye by gel filtration, e.g., by using desalting columns.

Acknowledgments The authors (VJ and $\mathrm{MH}$ ) thank ESA and the RW-TÜV foundation for financial support.

\section{References}

\section{Fluorescence Imaging}

Ale A, Ermolayev V, Deliolanis NC, et al. Fluorescence background subtraction technique for hybrid fluorescence molecular tomography/x-ray computed tomography imaging of a mouse model of early stage lung cancer. J Biomed Opt. 2013;18:56006. doi:10.1117/1. jbo.18.5.056006. 
Alfano RR, Demos SG, Gayen SK. Advances in optical imaging of biomedical media. Imaging Brain Struct Funct. 1997;820:248-71. doi:10.1111/j.1749-6632. 1997.tb46200.x.

Allen TJ, Beard PC. Pulsed near-infrared laser diode excitation system for biomedical photoacoustic imaging. Opt Lett. 2006a;31:3462-4. doi:10.1364/O1.31.003462.

Anumala UR, Gu JM, Lo Monte F, et al. Fluorescent rhodanine-3-acetic acids visualize neurofibrillary tangles in Alzheimer's disease brains. Bioorg Med Chem. 2013;21:5139-44. doi:10.1016/j.bmc.2013.06.039.

Arridge SR. Optical tomography in medical imaging. Inverse Prob. 1999;15:R41-93. doi:10.1088/ 0266-5611/15/2/022.

Backer MV, Gaynutdinov TI, Patel V, et al. Vascular endothelial growth factor selectively targets boronated dendrimers to tumor vasculature. Mol Cancer Ther. 2005;4:1423-9. doi:10.1158/1535-7163.Mct-05-0161.

Barthel H, Gertz HJ, Dresel S, et al. Cerebral amyloid-beta PET with florbetaben (F-18) in patients with Alzheimer's disease and healthy controls: a multicentre phase 2 diagnostic study. Lancet Neurol. 2011;10:42435. doi:10.1016/S1474-4422(11)70077-1.

Becker A, Riefke B, Ebert B, et al. Macromolecular contrast agents for optical imaging of tumors: comparison of indotricarbocyanine-labeled human serum albumin and transferrin. Photochem Photobiol. 2000;72:234 41. doi:10.1562/0031-8655(2000)072<0234:Mcafoi $>$ 2.0.Co;2.

Bendsoe N, Persson L, Johansson A, et al. Fluorescence monitoring of a topically applied liposomal temoporfin formulation and photodynamic therapy of nonpigmented skin malignancies. J Environ Pathol Toxicol oncol. 2007;26:117-26.

Bentzen EL, Tomlinson ID, Mason J, et al. Surface modification to reduce nonspecific binding of quantum dots in live cell assays. Bioconjug Chem. 2005;16:148894. doi:10.1021/Bc0502006.

Benya R, Quintana J, Brundage B. Adverse reactions to indocyanine green - a case-report and a review of the literature. Cathet Cardiovasc Diagn. 1989;17:231-3. doi:10.1002/ccd.1810170410.

Bertoncini CW, Celej MS. Small molecule fluorescent probes for the detection of amyloid self-assembly in vitro and in vivo. Curr Protein Pept Sc. 2011;12:206-20.

Bolander A, Kieser D, Voss C, et al. Bis(arylvinyl)pyrazines, -pyrimidines, and -pyridazines as imaging agents for tau fibrils and beta-amyloid plaques in Alzheimer's disease models. J Med Chem. 2012;55:9170-80. doi:10.1021/jm300653b.

Boot MJ, Westerberg CH, Sanz-Ezquerro J, et al. In vitro whole-organ imaging: 4D quantification of growing mouse limb buds. Nat Methods. 2008;5:609-12. doi:10.1038/Nmeth.1219.

Bremer C, Tung $\mathrm{CH}$, Weissleder R. In vivo molecular target assessment of matrix metalloproteinase inhibition. Nat Med. 2001a;7:743-8. doi:10.1038/89126.

Bruchez M, Moronne M, Gin P, et al. Semiconductor nanocrystals as fluorescent biological labels. Science. 1998;281:2013-6. doi:10.1126/science.281.5385.2013.
Buehler A, Herzog E, Razansky D, et al. Video rate optoacoustic tomography of mouse kidney perfusion. Opt Lett. 2010a;35:2475-7.

Busch C, Passon M, Wenzel M, et al. Detection of leukotriene receptor CysLT(1)R in inflammatory diseases by molecular imaging with near-infrared fluorescencebased contrast agents. Mol Imaging. 2011;10:81-90. doi:10.2310/7290.2010.00023.

Busch C, Schroter T, Grabolle M, et al. An in vivo spectral multiplexing approach for the cooperative imaging of different disease-related biomarkers with near-infrared fluorescent Forster resonance energy transfer probes. J Nucl Med. 2012;53:638-46. doi:10.2967/jnumed.111.094391.

Cammidge AN, Gopee H. Macrodiscotic triphenylenophthalocyanines. Chem Commun. 2002; 966-7. doi:10.1039/B200978a.

Chamberland D, Jiang YB, Wang XD. Optical imaging: new tools for arthritis. Integr Biol-UK. 2010;2:496509. doi:10.1039/B926506f.

Chan WCW, Maxwell DJ, Gao XH, et al. Luminescent quantum dots for multiplexed biological detection and imaging. Curr Opin Biotechnol. 2002;13:40-6. doi:10.1016/S0958-1669(02)00282-3.

Chang B, Yannuzzi LA, Ladas ID, et al. Choroidal neovascularization in 2nd eyes of patients with unilateral exudative age-related macular degeneration. Ophthalmology. 1995;102:1380-6.

Chen JQ, Tung CH, Mahmood U, et al. In vivo imaging of proteolytic activity in atherosclerosis. Circulation. 2002;105:2766-71. doi:10.1161/01. Cir.0000017860.20619.23.

Chen WT, Mahmood U, Weissleder R, et al. Arthritis imaging using a near-infrared fluorescence folatetargeted probe. Arthritis Res Ther. 2005;7:R310-7. doi:10.1186/Ar1483.

Cherrick GR, Stein SW, Leevy CM, et al. Indocyanine green - observations on its physical properties, plasma decay, and hepatic extraction. J Clin Invest. 1960;39:592-600. doi:10.1172/Jci104072.

Contag $\mathrm{CH}$, Bachmann $\mathrm{MH}$. Advances in vivo bioluminescence imaging of gene expression. Annu Rev Biomed Eng. 2002;4:235-60. doi:10.1146/annurev. bioeng.4.111901.093336.

Deguchi J, Aikawa M, Tung CH, et al. Inflammation in atherosclerosis - visualizing matrix metalloproteinase action in macrophages in vivo. Circulation. 2006;114:5562. doi:10.1161/Circulationaha.106.619056.

Dietzel F, Boettger MK, Dahlke K, et al. Assessment of rat antigen-induced arthritis and its suppression during glucocorticoid therapy by use of hemicyanine dye probes with different molecular weight in nearinfrared fluorescence optical imaging. Invest Radiol. 2013;48:729-37.

Dilek G, Akkaya EU. Novel squaraine signalling Zn(II) ions: three-state fluorescence response to a single input. Tetrahedron Lett. 2000;41:3721-4. doi:10.1016/ S0040-4039(00)00474-3.

Duncan R, Ringsdorf H, Satchi-Fainaro R. Polymer therapeutics: polymers as drugs, drug and protein conjugates and gene delivery systems: past, present 
and future opportunities. Adv Polym Sci. 2006;192:18. doi:10.1007/12_037.

Eckroat TJ, Mayhoub AS, Garneau-Tsodikova S. Amyloid-beta probes: review of structure-activity and brain-kinetics relationships. Beilstein J Org Chem. 2013;9:1012-44. doi:10.3762/Bjoc.9.116.

Eghtedari M, Oraevsky A, Copland JA, et al. High sensitivity of in vivo detection of gold nanorods using a laser optoacoustic imaging system. Nano Lett. 2007;7:1914-8. doi:10.1021/N1070557d.

Eisenblatter M, Ehrchen J, Varga G, et al. In vivo optical imaging of cellular inflammatory response in granuloma formation using fluorescence-labeled macrophages. J Nucl Med. 2009;50:1676-82. doi:10.2967/ jnumed.108.060707.

Frangioni JV. In vivo near-infrared fluorescence imaging. Curr Opin Chem Biol. 2003;7:626-34. doi:10.1016/j. cbpa.2003.08.007.

Galande AK, Hilderbrand SA, Weissleder R, et al. Enzyme-targeted fluorescent imaging probes on a multiple antigenic peptide core. J Med Chem. 2006;49:4715-20. doi:10.1021/Jm051001a.

Gao FP, Lin YX, Li LL, et al. Supramolecular adducts of squaraine and protein for noninvasive tumor imaging and photothermal therapy in vivo. Biomaterials. 2014;35:1004-14. doi:10.1016/j.biomaterials.2013. 10.039 .

Gonnert FA, Recknagel P, Hilger I, et al. Hepatic excretory function in sepsis: implications from biophotonic analysis of transcellular xenobiotic transport in a rodent model. Crit Care. 2013;17:R67. doi:10.1186/ cc12606.

Gotoh K, Yamada T, Ishikawa O, et al. HOW I DO IT a novel image-guided surgery of hepatocellular carcinoma by indocyanine green fluorescence imaging navigation. J Surg Oncol. 2009;100:75-9. doi:10.1002/ Jso. 21272 .

Gremse F, Theek B, Kunjachan S, et al. Absorption reconstruction improves biodistribution assessment of fluorescent nanoprobes using hybrid fluorescencemediated tomography. Theranostics. 2014;4:960-71. doi:10.7150/thno.9293.

Gu JM, Anumala UR, Heyny-von Haussen R, et al. Design, synthesis and biological evaluation of trimethine cyanine dyes as fluorescent probes for the detection of tau fibrils in Alzheimer's disease brain and olfactory epithelium. Chemmedchem. 2013;8:891-7. doi:10.1002/cmdc.201300090.

Haedicke K, Grafe S, Lehmann F, et al. Multiplexed in vivo fluorescence optical imaging of the therapeutic efficacy of photodynamic therapy. Biomaterials. 2013;34:10075-83. doi:10.1016/j.biomaterials.2013. 08.087.

Haedicke K, Kozlova D, Gräfe S, et al. Multifunctional calcium phosphate nanoparticles for combining nearinfrared fluorescence imaging and photodynamic therapy. Acta Biomater. 2015;14:197-207.

Hamann FM, Brehm R, Pauli J, et al. Controlled modulation of serum protein binding and biodistribution of asymmetric cyanine dyes by variation of the number of sulfonate groups. Mol Imaging. 2011;10:258-69. doi:10.2310/7290.2011.00005.

Hansch A, Frey O, Sauner D, et al. In vivo imaging of experimental arthritis with near-infrared fluorescence. Arthritis Rheum. 2004;50:961-7. doi:10.1002/Art.20112.

Hardman R. A toxicologic review of quantum dots: toxicity depends on physicochemical and environmental factors. Environ Health Perspect. 2006;114:165-72. doi:10.1289/Ehp.8284.

He XX, Chen JY, Wang KM, et al. Preparation of luminescent Cy5 doped core-shell SFNPs and its application as a near-infrared fluorescent marker. Talanta. 2007;72:1519-26. doi:10.1016/j.talanta.2007.01.069.

Hilger I, Leistner Y, Berndt A, et al. Near-infrared fluorescence imaging of HER-2 protein over-expression in tumour cells. Eur Radiol. 2004;14:1124-9. doi:10.1007/s00330-004-2257-9.

Hintersteiner M, Enz A, Frey P, et al. In vivo detection of amyloid-beta deposits by near-infrared imaging using an oxazine-derivative probe. Nat Biotechnol. 2005;23:577-83. doi:10.1038/nbt1085.

Ho JD, Tsai RJF, Chen SN, et al. Cytotoxicity of indocyanine green on retinal pigment epithelium - Implications for macular hole surgery. Arch Ophthalmol-Chic. 2003;121:1423-9. doi:10.1001/archopht.121.10.1423.

Hu X, Wang Q, Liu Y, et al. Optical imaging of articular cartilage degeneration using near-infrared dipicolylamine probes. Biomaterials. 2014;35:7511-21. doi:10.1016/j.biomaterials.2014.05.042.

Huisken J, Swoger J, Del Bene F, et al. Optical sectioning deep inside live embryos by selective plane illumination microscopy. Science. 2004;305:1007-9. doi:10.1126/science.1100035.

Ikagawa $\mathrm{H}$, Yoneda M, Iwaki M, et al. Chemical toxicity of indocyanine green damages retinal pigment epithelium. Invest Ophth Vis Sci. 2005;46:2531-9. doi:10.1167/Iovs-04-1521.

Ishizawa T, Fukushima N, Shibahara J, et al. Real-time identification of liver cancers by using indocyanine green fluorescent imaging. Cancer. 2009;115: 2491-504. doi:10.1002/Cncr.24291.

Jackson SP. Arterial thrombosis-insidious, unpredictable and deadly. Nat Med. 2011;17:1423-36. doi:10.1038/ Nm.2515.

JafferFA, KimDE, QuintiL, etal. Optical visualization of cathep$\sin \mathrm{K}$ activity in atherosclerosis with a novel, protease-activatable fluorescence sensor. Circulation. 2007;115:2292-8. doi:10.1161/Circulationaha.106.660340.

Jamar F, Houssiau FA, Devogelaer JP, et al. Scintigraphy using a technetium 99m-labelled anti-E-selectin Fab fragment in rheumatoid arthritis. Rheumatology. 2002;41:53-61. doi:10.1093/rheumatology/41.1.53.

Jobsis FF. Noninvasive, infrared monitoring of cerebral and myocardial oxygen sufficiency and circulatory parameters. Science. 1977;198:1264-7. doi:10.1126/ science.929199.

Kairdolf BA, Mancini MC, Smith AM, et al. Minimizing nonspecific cellular binding of quantum dots with hydroxyl-derivatizied surface coatings. Anal Chem. 2008;80:3029-34. doi:10.1021/Ac800068q. 
Kim JS, Kodagahally R, Strekowski L, et al. A study of intramolecular H-complexes of novel bis(heptamethine cyanine) dyes. Talanta. 2005;67:947-54. doi:10.1016/j. talanta.2005.04.025.

Kolkman RGM, Klaessens JHGM, Hondebrink E, et al. Photoacoustic determination of blood vessel diameter. Phys Med Biol. 2004;49:4745-56. doi:10.1088/00319155/49/20/006. Pii S0031-9155(04)83132-4.

Koronyo-Hamaoui M, Koronyo Y, Ljubimov AV, et al. Identification of amyloid plaques in retinas from Alzheimer's patients and noninvasive in vivo optical imaging of retinal plaques in a mouse model. Neuroimage. 2011;54:S204-17. doi:10.1016/j.neuroimage.2010.06.020.

Kossatz S, Behe M, Mansi R, et al. Multifactorial diagnostic NIR imaging of CCK2R expressing tumors. Biomaterials. 2013;34:5172-80. doi:10.1016/j. biomaterials.2013.03.073.

Kovar JL, Simpson MA, Schutz-Geschwender A, et al. A systematic approach to the development agents for optical imaging of mouse of fluorescent contrast cancer models. Anal Biochem. 2007;367:1-12. doi:10.1016/J.Ab.2007.04.011.

Lai WF, Chang $\mathrm{CH}$, Tang Y, et al. Early diagnosis of osteoarthritis using cathepsin B sensitive near-infrared fluorescent probes. Osteoarthr Cartil. 2004;12:239_ 44. doi:10.1016/j.joca.2003.11.005.

Lakowicz JR. Principles of fluorescence spectroscopy. 2nd ed. New York: Kluwer Academic/Plenum Publishers; 1999.

Landsman MLJ, Kwant G, Mook GA, et al. Lightabsorbing properties, stability, and spectral stabilization of indocyanine green. J Appl Physiol. 1976;40: 575-83.

Lim YT, Kim S, Nakayama A, et al. Selection of quantum dot wavelengths for biomedical assays and imaging. Mol Imaging. 2003;2:50-64.

Lipinski MJ, Amirbekian V, Frias JC, et al. MRI to detect atherosclerosis with gadolinium-containing immunomicelles targeting the macrophage scavenger receptor. Magn Reson Med. 2006;56:601-10. doi:10.1002/ Mrm.20995.

Liu CC, Gu YQ. Noninvasive optical imaging of Staphylococcus aureus infection in vivo using an antimicrobial petide fragment based near-infrared fluorescent probes. J Innov Opt Heal Sci. 2013;6:Artn 1350026. doi: 10.1142/S1793545813500260.

Liu TC, Wu LY, Hopkins MR, et al. A targeted low molecular weight near-infrared fluorescent probe for prostate cancer. Bioorg Med Chem Lett. 2010;20:7124-6. doi:10.1016/j.bmcl.2010.09.057.

Luo SL, Zhang EL, Su YP, et al. A review of NIR dyes in cancer targeting and imaging. Biomaterials. 2011a;32:712738. doi:10.1016/j.biomaterials.2011.06.024.

Maeda H, Bharate GY, Daruwalla J. Polymeric drugs for efficient tumor-targeted drug delivery based on EPReffect. Eur J Pharm Biopharm. 2009;71:409-19. doi:10.1016/j.ejpb.2008.11.010.

Marshall D, Haskard DO. Clinical overview of leukocyte adhesion and migration: where are we now? Semin Immunol. 2002;14:133-40. doi:10.1006/smim.2001.0350.
Messerli SM, Prabhakar S, Tang Y, et al. A novel method for imaging apoptosis using a caspase-1 near-infrared fluorescent probe. Neoplasia. 2004;6:95-105. doi:10.1593/Neo.03214.

Mishra A, Behera RK, Behera PK, et al. Cyanines during the 1990s: a review. Chem Rev. 2000;100:1973-2011. doi:10.1021/Cr990402t.

Moore GE, Peyton WT, French LA, et al. The clinical use of fluorescein in neurosurgery - the localization of brain tumors. J Neurosurg. 1948;5:392-8. doi:10.3171/ jns.1948.5.4.0392.

Munder A, Wolbeling F, Klockgether J, et al. In vivo imaging of bioluminescent Pseudomonas aeruginosa in an acute murine airway infection model. Pathog Dis. 2014;72:74-7. doi:10.1111/2049-632x.12184.

Nahrendorf M, Sosnovik DE, Waterman P, et al. Dual channel optical tomographic imaging of leukocyte recruitment and protease activity in the healing myocardial infarct. Circ Res. 2007;100:1218-25. doi:10.1161/01.Res.0000265064.46075.31.

Nikoobakht B, El-Sayed MA. Preparation and growth mechanism of gold nanorods (NRs) using seedmediated growth method. Chem Mater. 2003;15:195762. doi:10.1021/Cm0207321.

Ntziachristos V. Going deeper than microscopy: the optical imaging frontier in biology. Nat Methods. 2010;7:603-14. doi:10.1038/Nmeth.1483.

Ntziachristos V. Clinical translation of optical and optoacoustic imaging. Philos T R Soc A. 2011;369:466678. doi:10.1098/rsta.2011.0270.

Ntziachristos V, Bremer C, Tung C, et al. Imaging cathepsin B up-regulation in HT-1080 tumor models using fluorescence-mediated molecular tomography (FMT). Acad Radiol. 2002;9:S323-5. doi:10.1016/ S1076-6332(03)80217-9.

Ogawa M, Kosaka N, Choyke PL, et al. Tumor-specific detection of an optically targeted antibody combined with a quencher-conjugated neutravidin "quencherchaser": a dual "quench and chase" strategy to improve target to nontarget ratios for molecular imaging of cancer. Bioconjug Chem. 2009;20:147-54. doi:10.1021/bc8003765.

Ohnishi S, Lomnes SJ, Laurence RG, et al. Organic alternatives to quantum dots for intraoperative nearinfrared fluorescent sentinel lymph node mapping. Mol Imaging. 2005;4:172-81.

Okamura N, Mori M, Furumoto S, et al. In vivo detection of amyloid plaques in the mouse brain using the nearinfrared fluorescence probe THK-265. J Alzheimers Dis. 2011;23:37-48. doi:10.3233/Jad-2010-100270.

Osterman HL, Schutz-Geschwender A. Seeing beyond the visible with IRDye infrared dyes. LI-COR Biosci. 2007:1-8.

Oswald B, Lehmann F, Simon L, et al. Red laser-induced fluorescence energy transfer in an immunosystem. Anal Biochem. 2000;280:272-7. doi:10.1006/abio.2000.4553.

Panchuk-Voloshina N, Haugland RP, Bishop-Stewart J, et al. Alexa dyes, a series of new fluorescent dyes that yield exceptionally bright, photostable conjugates. J Histochem Cytochem. 1999;47:1179-88. 
Pauli J, Vag T, Haag R, et al. An in vitro characterization study of new near infrared dyes for molecular imaging. Eur J Med Chem. 2009a;44:3496-503. doi:10.1016/j.ejmech.2009.01.019.

Pauli J, Grabolle M, Brehm R, et al. Suitable labels for molecular imaging - influence of dye structure and hydrophilicity on the spectroscopic properties of IgG conjugates. Bioconjug Chem. 2011;22:1298-308. doi:10.1021/bc1004763.

Peng XJ, Song FL, Lu E, et al. Heptamethine cyanine dyes with a large stokes shift and strong fluorescence: a paradigm for excited-state intramolecular charge transfer. J Am Chem Soc. 2005;127:4170-1. doi:10.1021/Ja043413z.

Pham W, Medarova Z, Moore A. Synthesis and application of a water-soluble near-infrared dye for cancer detection using optical imaging. Bioconjug Chem. 2005;16:735-40. doi:10.1021/Bc049700+.

Ploemen IHJ, Prudencio M, Douradinha BG, et al. Visualisation and quantitative analysis of the Rodent malaria liver stage by real time imaging. Plos One. 2009; 4:Artn E7881. doi:10.1371/Journal. Pone.0007881.

Razansky D, Vinegoni C, Ntziachristos V. Multispectral photoacoustic imaging of fluorochromes in small animals. Opt Lett. 2007;32:2891-3. doi:10.1364/ O1.32.002891.

Recknagel P, Gonnert FA, Westermann M, et al. Liver dysfunction and phosphatidylinositol-3-kinase signalling in early sepsis: experimental studies in rodent models of peritonitis. Plos Med. 2012;9:e1001338. doi:10.1371/journal.pmed.1001338.

Rickert EL, Oriana S, Hartman-Frey C, et al. Synthesis and characterization of fluorescent 4-hydroxytamoxifen conjugates with unique antiestrogenic properties. Bioconjug Chem. 2010;21:903-10. doi:10.1021/Bc900461h.

Sakatani K, KashiwasakeJibu M, Wang S, et al. Noninvasive optical imaging of the subarachnoid space and cerebrospinal fluid pathways based on nearinfrared fluorescence. J Neurosurg. 1997;87:738-45. doi:10.3171/jns.1997.87.5.0738.

Schafer VS, Hartung W, Hoffstetter P, et al. Quantitative assessment of synovitis in patients with rheumatoid arthritis using fluorescence optical imaging. Arthritis Res Ther. 2013;15:Artn R124 doi:10.1186/Ar4304.

Schotland JC, Leigh JS. Photon diffusion imaging. Faseb J. 1992;6:A446-A.

Serhan CN, Chiang N, Van Dyke TE. Resolving inflammation: dual anti-inflammatory and pro-resolution lipid mediators. Nat Rev Immunol. 2008;8:349-61. doi:10.1038/Nri2294.

Simon GH, Daldrup-Link HE, Kau J, et al. Optical imaging of experimental arthritis using allogeneic leukocytes labeled with a near-infrared fluorescent probe. Eur J Nucl Med Mol I. 2006;33:998-1006. doi:10.1007/s00259-006-0081-y.

SkrivanovaK, SkorpikovaJ, SvihalekJ, etal.Photochemical properties of a potential photosensitiser indocyanine green in vitro. J Photoch Photobio B. 2006;85:150-4. doi:10.1016/j.jphotobiol.2006.06.004.
Sparks JS, Schelly RC, Smith WL, et al. The covert world of fish biofluorescence: a phylogenetically widespread and phenotypically variable phenomenon. Plos One. 2014;9:ARTN e83259. doi:10.1371/journal. pone.0083259.

Tang EN, Nair A, Baker DW, et al. In vivo imaging of infection using a bacteria-targeting optical nanoprobe. J Biomed Nanotechnol. 2014;10:856-63. doi:10.1166/ jbn.2014.1852.

Tansi FL, Ruger R, Rabenhold M, et al. Liposomal encapsulation of a near-infrared fluorophore enhances fluorescence quenching and reliable whole body optical imaging upon activation in vivo. Small. 2013;9:365969. doi:10.1002/smll.201203211.

Taqueti VR, Jaffer FA. High-resolution molecular imaging via intravital microscopy: illuminating vascular biology in vivo. Integr Biol-UK. 2013;5:278-90. doi:10.1039/C2ib20194a.

te Velde EA, Veerman T, Subramaniam V, et al. The use of fluorescent dyes and probes in surgical oncology. Ejso-Eur J Surg Onc. 2010;36:6-15. doi:10.1016/j. ejso.2009.10.014.

Tsung CK, Kou XS, Shi QH, et al. Selective shortening of single-crystalline gold nanorods by mild oxidation. J Am Chem Soc. 2006;128:5352-3. doi:10.1021/ $\mathrm{Ja060447t}$.

Tung $\mathrm{CH}$. Fluorescent peptide probes for in vivo diagnostic imaging. Biopolymers. 2004;76:391-403. doi:10.1002/Bip.20139.

Tung $\mathrm{CH}$, Lin $\mathrm{YH}$, Moon WK, et al. A receptor-targeted near-infrared fluorescence probe for in vivo tumor imaging. Chembiochem. 2002;3:784-6. doi:10.1002/1439-7633(20020802)3:8<784::AidCbic784>3.0.Co;2-X.

van Dam GM, Themelis G, Crane LMA, et al. Intraoperative tumor-specific fluorescence imaging in ovarian cancer by folate receptor-alpha targeting: first in-human results. Nat Med. 2011;17:1315-U202. doi:10.1038/Nm.2472.

van der Poel HG, Buckle T, Brouwer OR, et al. Intraoperative laparoscopic fluorescence guidance to the sentinel lymph node in prostate cancer patients: clinical proof of concept of an integrated functional imaging approach using a multimodal tracer. Eur Urol. 2011;60:826-33. doi:10.1016/j.eururo.2011.03.024.

Volkova KD, Kovalska VB, Tatarets AL, et al. Spectroscopic study of squaraines as protein-sensitive fluorescent dyes. Dyes Pigm. 2007;72:285-92. doi:10.1016/j.dyepig.2005.09.007.

Walling MA, Novak JA, Shepard JRE. Quantum dots for live cell and in vivo imaging. Int $\mathrm{J}$ Mol Sci. 2009;10:441-91. doi:10.3390/Ijms 10020441.

Wang W, Ke S, Kwon S, et al. A new optical and nuclear dual-labeled imaging agent targeting interleukin 11 receptor alpha-chain. Bioconjug Chem. 2007a;18:397402. doi:10.1021/Bc0602679.

Wang R, Yu CW, Yu FBA, et al. Molecular fluorescent probes for monitoring $\mathrm{pH}$ changes in living cells. Trac-Trend Anal Chem. 2010a;29:1004-13. doi:10.1016/j.trac.2010.05.005. 
Weissleder R, Ntziachristos V. Shedding light onto live molecular targets. Nat Med. 2003;9:123-8. doi:10.1038/Nm0103-123.

White AG, Fu N, Leevy WM, et al. Optical imaging of bacterial infection in living mice using deep-Red fluorescent squaraine rotaxane probes. Bioconjug Chem. 2010;21:1297-304. doi:10.1021/bc1000998.

Wu X, Liu H, Liu J, et al. Immunofluorescent labeling of cancer marker Her2 and other cellular targets with semiconductor quantum dots. Nat Biotechnol. 2003;21:41-6. doi:10.1038/nbt764.

Yodh A, Chance B. Spectroscopy and imaging with diffusing light. Phys Today. 1995;48:34-40. doi:10.1063/ 1.881445 .

Yoo H, Kim JW, Shishkov M, et al. Intra-arterial catheter for simultaneous microstructural and molecular imaging in vivo. Nat Med. 2011;17:1680-U202. doi:10.1038/ Nm.2555.

Zemp RJ, Bitton R, Li ML, et al. Photoacoustic imaging of the microvasculature with a high-frequency ultrasound array transducer. J Biomed Opt. 2007;12:Artn 010501. doi:10.1117/1.2709850.

Zhou LC, Zhao GJ, Liu JF, et al. The charge transfer mechanism and spectral properties of a near-infrared heptamethine cyanine dye in alcoholic and aprotic solvents. J Photoch Photobio A. 2007;187:305-10. doi:10.1016/j.jphotochem.2006.10.027.

\section{Bioluminescence Imaging}

Adamczak JM, et al. In vivo bioluminescence imaging of vascular remodeling after stroke. Front Cell Neurosci. 2014;8:274.

Brakenhielm E, et al. Modulating metastasis by a lymphangiogenic switch in prostate cancer. Int J Cancer. 2007;121(10):2153-61.

Burns-Guydish SM, et al. Monitoring age-related susceptibility of young mice to oral Salmonella enterica serovar Typhimurium infection using an in vivo murine model. Pediatr Res. 2005;58(1):153-8.

Chewning $\mathrm{JH}$, et al. Bioluminescence-based visualization of CD4 T cell dynamics using a T lineage-specific luciferase transgenic model. BMC Immunol. 2009;10:44.

Close DM, et al. In vivo bioluminescent imaging (BLI): noninvasive visualization and interrogation of biological processes in living animals. Sens (Basel). 2011; 11(1):180-206.

Contag PR, et al. Bioluminescent indicators in living mammals. Nat Med. 1998;4(2):245-7.

Cowey $\mathrm{S}$, et al. Breast cancer metastasis to bone: evaluation of bioluminescent imaging and microSPECT/CT for detecting bone metastasis in immunodeficient mice. Clin Exp Metastasis. 2007;24(5):389-401.

Cruz-Monserrate Z, et al. Targeting pancreatic ductal adenocarcinoma acidic microenvironment. Sci Rep. 2014;4:4410.

de Wet JR, et al. Cloning of firefly luciferase cDNA and the expression of active luciferase in Escherichia coli. Proc Natl Acad Sci U S A. 1985;82(23):7870-3.
Desjardin DE, Oliveira AG, Stevani CV. Fungi bioluminescence revisited. Photochem Photobiol Sci. 2008; 7(2):170-82.

Haddock SH, et al. Bioluminescent and red-fluorescent lures in a deep-sea siphonophore. Science. 2005; 309(5732):263.

Haddock SH, Moline MA, Case JF. Bioluminescence in the sea. Ann Rev Mar Sci. 2010;2:443-93.

Hastings JW. Chemistries and colors of bioluminescent reactions: a review. Gene. 1996;173(1 Spec No): 5-11.

Hiler DJ, Greenwald ML, Geusz ME. Imaging gene expression in live transgenic mice after providing luciferin in drinking water. Photochem Photobiol Sci. 2006;5(11):1082-5.

Ignowski JM, Schaffer DV. Kinetic analysis and modeling of firefly luciferase as a quantitative reporter gene in live mammalian cells. Biotechnol Bioeng. 2004;86(7): 827-34.

Inoue Y, et al. Comparison of subcutaneous and intraperitoneal injection of D-luciferin for in vivo bioluminescence imaging. Eur $\mathrm{J}$ Nucl Med Mol Imaging. 2009;36(5):771-9.

Ishikawa TO, et al. Imaging cyclooxygenase-2 (Cox-2) gene expression in living animals with a luciferase knock-in reporter gene. Mol Imaging Biol. 2006; 8(3):171-87.

Iyer $\mathrm{M}$, et al. Non-invasive imaging of a transgenic mouse model using a prostate-specific two-step transcriptional amplification strategy. Transgenic Res. 2005; 14(1):47-55.

Iyer $\mathrm{M}$, et al. Bioluminescence imaging of systemic tumor targeting using a prostate-specific lentiviral vector. Hum Gene Ther. 2006;17(1):125-32.

Kanerva A, et al. Enhanced therapeutic efficacy for ovarian cancer with a serotype 3 receptor-targeted oncolytic adenovirus. Mol Ther. 2003;8(3):449-58.

Keyaerts M, et al. Dynamic bioluminescence imaging for quantitative tumour burden assessment using IV or IP administration of D: -luciferin: effect on intensity, time kinetics and repeatability of photon emission. Eur J Nucl Med Mol Imaging. 2008;35(5):999-1007.

Keyaerts M, et al. Inhibition of firefly luciferase by general anesthetics: effect on in vitro and in vivo bioluminescence imaging. PLoS One. 2012;7(1):e30061.

Laxman B, et al. Noninvasive real-time imaging of apoptosis. Proc Natl Acad Sci U S A. 2002;99(26):16551-5.

Li J, et al. Cage the firefly luciferin! - a strategy for developing bioluminescent probes. Chem Soc Rev. 2013a;42(2):662-76.

Maes W, et al. In vivo bioluminescence imaging in an experimental mouse model for dendritic cell based immunotherapy against malignant glioma. J Neurooncol. 2009;91(2):127-39.

Mendes LF, et al. Influence of culture conditions on mycelial growth and bioluminescence of Gerronema viridilucens. FEMS Microbiol Lett. 2008;282(1):132-9.

Miller SD, et al. Detection of a bioluminescent milky sea from space. Proc Natl Acad Sci U S A. 2005;102(40):14181-4. 
Moreno JC, et al. Effect of the relative optical air mass and the clearness index on solar erythemal UV irradiance. J Photochem Photobiol B. 2014;138:92-8.

Paulmurugan R, Umezawa Y, Gambhir SS. Noninvasive imaging of protein-protein interactions in living subjects by using reporter protein complementation and reconstitution strategies. Proc Natl Acad Sci U S A. 2002;99(24):15608-13.

Poisson J. Raphael Dubois, from pharmacy to bioluminescence. Rev Hist Pharm (Paris). 2010;58(365):51-6.

Rehemtulla A, Taneja N, Ross BD. Bioluminescence detection of cells having stabilized $\mathrm{p} 53$ in response to a genotoxic event. Mol Imaging. 2004;3(1):63-8.

Sabag AD, et al. Semaphorin-3D and semaphorin-3E inhibit the development of tumors from glioblastoma cells implanted in the cortex of the brain. PLoS One. 2012;7(8):e42912.

Sadikot RT, et al. Pathogen-host interactions in Pseudomonas aeruginosa pneumonia. Am J Respir Crit Care Med. 2005;171(11):1209-23.

Saha D, et al. In vivo bioluminescence imaging of tumor hypoxia dynamics of breast cancer brain metastasis in a mouse model. J Vis Exp. 2011;56.

Siragusa GR, et al. Real-time monitoring of Escherichia coli $\mathrm{O} 157: \mathrm{H} 7$ adherence to beef carcass surface tissues with a bioluminescent reporter. Appl Environ Microbiol. 1999;65(4):1738-45.

Soling A, et al. A dual function fusion protein of Herpes simplex virus type 1 thymidine kinase and firefly luciferase for noninvasive in vivo imaging of gene therapy in malignant glioma. Genet Vaccines Ther. 2004;2(1):7.

Tiffen JC, et al. Luciferase expression and bioluminescence does not affect tumor cell growth in vitro or in vivo. Mol Cancer. 2010;9:299.

Wang W, El-Deiry WS. Bioluminescent molecular imaging of endogenous and exogenous p53-mediated transcription in vitro and in vivo using an HCT116 human colon carcinoma xenograft model. Cancer Biol Ther. 2003;2(2):196-202.

Warrant EJ, Locket NA. Vision in the deep sea. Biol Rev Camb Philos Soc. 2004;79(3):671-712.

Wiles S, et al. Organ specificity, colonization and clearance dynamics in vivo following oral challenges with the murine pathogen Citrobacter rodentium. Cell Microbiol. 2004;6(10):963-72.

Wiles $\mathrm{S}$, et al. In vivo bioluminescence imaging of the murine pathogen Citrobacter rodentium. Infect Immun. 2006;74(9):5391-6.

Zhao RL, et al. A new species of bird's nest fungi: characterisation of Cyathus subglobisporus sp. nov. based on morphological and molecular data. Persoonia. 2008;21:71-6.

Zinn KR, et al. Noninvasive bioluminescence imaging in small animals. ILAR J. 2008;49(1):103-15.

\section{Optical Coherence Tomography (OCT)}

Abbe E. Beiträge zur Theorie des Mikroskops und der mikroskopischen Wahrnehmung. Archiv F Mikrosk Anatomie. 1873;9:413-8.
Al-Qaisi MK, Akkin T. Swept-source polarizationsensitive optical coherence tomography based on polarization-maintaining fiber. Opt Express. 2010;18: 3392-403.

Anderson RR, Parrish JA. The optics of human-skin. J Invest Dermatol. 1981;77:13-9.

Bachmann AH, Villiger ML, Blatter C, Lasser T, Leitgeb RA. Resonant Doppler flow imaging and optical vivisection of retinal blood vessels. Opt Express. 2007;15:408-22.

Bail MA, Haeusler G, Herrmann JM, Lindner MW, Ringler R. Optical coherence tomography with the "spectral radar": fast optical analysis in volume scatterers by short-coherence interferometry. In: Benaron DA, Chance B, Mueller GJ, editors. Vienna: SPIE; 1996. p. 298-303.

Bailey TJ, Davis DH, Vance JE, Hyde DR. Spectraldomain optical coherence tomography as a noninvasive method to assess damaged and regenerating adult zebrafish retinas. Invest Ophthalmol Vis Sci. 2012;53: 3126-38.

Bajraszewski T, Wojtkowski M, Szkulmowski M, et al. Improved spectral optical coherence tomography using optical frequency comb. Opt Express. 2008;16: 4163-76.

Bayleyegn MD, Makhlouf H, Crotti C, Plamann K, Dubois A. Ultrahigh resolution spectral-domain optical coherence tomography at $1.3 \mathrm{mu}$ m using a broadband superluminescent diode light source. Opt Commun. 2012;285:5564-9.

Bell BA, Vincent KL, Bourne N, Vargas G, Motamedi M. Optical coherence tomography for assessment of microbicide safety in a small animal model. J Biomed Opt. 2013;18:046010.

Bouma BE, Tearney GJ. Handbook of optical coherence tomography. New York: Marcel Dekker; 2002.

Bukowska D, Szkulmowska A, Grulkowski I, Tamborski S, Szkulmowski M, Leitgeb R, Kowalczyk A, Wojtkowski M. Observation of blood optical inhomogeneity using joint spectral and time domain OCT. 2010; 755412-5.

Cang H, Sun T, Li ZY, et al. Gold nanocages as contrast agents for spectroscopic optical coherence tomography. Opt Lett. 2005;30:3048-50.

Chan AC, Srinivasan VJ, Lam EY. Maximum likelihood Doppler frequency estimation under decorrelation noise for quantifying flow in optical coherence tomography. IEEE Trans Med Imaging. 2014;33:1313-23.

Chinn SR, Swanson EA, Fujimoto JG. Optical coherence tomography using a frequency-tunable optical source. Opt Lett. 1997;22:340-2.

Choma M, Sarunic M, Yang C, Izatt J. Sensitivity advantage of swept source and Fourier domain optical coherence tomography. Opt Express. 2003;11:2183-9.

Cimalla P, Walther J, Mehner M, Cuevas M, Koch E. Simultaneous dual-band optical coherence tomography in the spectral domain for high resolution in vivo imaging. Opt Express. 2009;17:19486-500.

Cimalla P, Walther J, Mittasch M, Koch E. Shear flowinduced optical inhomogeneity of blood assessed in vivo and in vitro by spectral domain optical 
coherence tomography in the 1.3 mum wavelength range. J Biomed Opt. 2011a;16:116020.

Cimalla P, Burkhardt A, Walther J et al. Non-invasive imaging and monitoring of rodent retina using simultaneous dual-band optical coherence tomography. Proc. SPIE. 2011b;7889:788909-788909.

Cimalla P, Gaertner M, Weber A, et al. A hybrid imaging system for simultaneous ophthalmic optical coherence tomography and dual-channel fluorescence detection in small animal models. Biomed Tech. 2012;57: 515-8.

de Boer JF, Milner TE, van Gemert MJC, Nelson JS. Twodimensional birefringence imaging in biological tissue by polarization-sensitive optical coherence tomography. Opt Lett. 1997;22:934-6.

de Boer JF, Cense B, Park BH, et al. Improved signal-tonoise ratio in spectral-domain compared with timedomain optical coherence tomography. Opt Lett. 2003;28:2067-9.

Drexler W, Fujimoto JG. State-of-the-art retinal optical coherence tomography. Prog Retin Eye Res. 2008;27: 45-88.

Dubois A, Vabre L, Boccara AC, Beaurepaire E. Highresolution full-field optical coherence tomography with a Linnik microscope. Appl Opt. 2002;41:805-12.

Duma VF, Lee KS, Meemon P, Rolland JP. Experimental investigations of the scanning functions of galvanometer-based scanners with applications in OCT. Appl Opt. 2011;50:5735-49.

Eigenwillig CM, Biedermann BR, Wieser W, Huber R. Wavelength swept amplified spontaneous emission source. Opt Express. 2009;17:18794-807.

Faber DJ, van Leeuwen TG. Are quantitative attenuation measurements of blood by optical coherence tomography feasible? Opt Lett. 2009;34:1435-7.

Faber DJ, Mik EG, Aalders MC, van Leeuwen TG. Toward assessment of blood oxygen saturation by spectroscopic optical coherence tomography. Opt Lett. 2005; 30:1015-7.

Fernández EJ, Hermann B, Povazay B, et al. Ultrahigh resolution optical coherence tomography and pancorrection for cellular imaging of the living human retina. Opt Express. 2008;16:11083-94.

Fischer MD, Huber G, Feng Y, et al. In vivo assessment of retinal vascular wall dimensions. Invest Ophthalmol Vis Sci. 2010;51:5254-9.

Fleming CP, Eckert J, Halpern EF, Gardecki JA, Tearney GJ. Depth resolved detection of lipid using spectroscopic optical coherence tomography. Biomed Opt Express. 2013;4:1269-84.

Gaertner M, Weber A, Cimalla P, KÂttig F, Brand M, Koch E. Towards a comprehensive eye model for zebrafish retinal imaging using full range spectral domain optical coherence tomography. p. $89342 \mathrm{H}-89342 \mathrm{H}$, International Society for Optics and Photonics. 2014.

Gao W. Dispersion properties of grating-based rapid scanning optical delay lines. Appl Opt. 2007;46:986-92.

Garini Y, Young IT, McNamara G. Spectral imaging: principles and applications. Cytometry A. 2006;69:735-47.

Goodman JW. Statistical optics. New York: Wiley; 1985.
Gora M, Karnowski K, Szkulmowski M, et al. Ultra highspeed swept source OCT imaging of the anterior segment of human eye at $200 \mathrm{kHz}$ with adjustable imaging range. Opt Express. 2009;17:14880-94.

Götzinger E, Baumann B, Pircher M, Hitzenberger CK. Polarization maintaining fiber based ultra-high resolution spectral domain polarization sensitive optical coherence tomography. Opt Express. 2009;17:22704-17.

Hariri S, Moayed AA, Dracopoulos A, et al. Limiting factors to the OCT axial resolution for in-vivo imaging of human and rodent retina in the $1060 \mathrm{~nm}$ wavelength range. Opt Express. 2009;17:24304-16.

Hartl I, Li XD, Chudoba C, et al. Ultrahigh-resolution optical coherence tomography using continuum generation in an air-silica microstructure optical fiber. Opt Lett. 2001;26:608-10.

Hecht E. Optik. München: Oldenbourg; 2005.

Hee MR, Huang D, Swanson EA, Fujimoto JG. Polarizationsensitive low-coherence reflectometer for birefringence characterization and ranging. J Opt Soc Am B. 1992;9: 903-8.

Hee MR, Izatt JA, Swanson EA, Fujimoto JG. Femtosecond transillumination tomography in thick tissues. Opt Lett. 1993;18:1107-9.

Hering E, Martin R. Photonik: Grundlagen, Technologie und Anwendung. Berlin: Springer; 2006.

Hitzenberger C, Goetzinger E, Sticker M, Pircher M, Fercher A. Measurement and imaging of birefringence and optic axis orientation by phase resolved polarization sensitive optical coherence tomography. Opt Express. 2001;9:780-90.

Horio N, Kachi S, Hori K, et al. Progressive change of optical coherence tomography scans in retinal degeneration slow mice. Arch Ophthalmol. 2001;119:1329-32.

$\mathrm{Hu} \mathrm{Z}$, Rollins AM. Fourier domain optical coherence tomography with a linear-in-wavenumber spectrometer. Opt Lett. 2007;32:3525-7.

Huang D, Swanson EA, Lin CP, et al. Optical coherence tomography. Science. 1991;254:1178-81.

Huber G, Beck SC, Grimm C, et al. Spectral domain optical coherence tomography in mouse models of retinal degeneration. Invest Ophthalmol Vis Sci. 2009;50:5888-95.

Huber G, Heynen S, Imsand C, et al. Novel rodent models for macular research. PLoS One. 2010;5:e13403.

Jaedicke V, Agcaer S, Robles FE, et al. Comparison of different metrics for analysis and visualization in spectroscopic optical coherence tomography. Biomed Opt Express. 2013;4:2945-61.

Jian Y, Zawadzki RJ, Sarunic MV. Adaptive optics optical coherence tomography for in vivo mouse retinal imaging. J Biomed Opt. 2013;18:056007-056007.

John R, Rezaeipoor R, Adie SG, et al. In vivo magnetomotive optical molecular imaging using targeted magnetic nanoprobes. Proc Natl Acad Sci. 2010;107: 8085-90.

Kalkman J, Bykov AV, Streekstra GJ, van Leeuwen TG. Multiple scattering effects in Doppler optical coherence tomography of flowing blood. Phys Med Biol. 2012;57:1907-17.

Kartakoullis A, Bousi E, Pitris C. Scatterer size-based analysis of optical coherence tomography images 
using spectral estimation techniques. Opt Express. 2010;18:9181-91.

Kasseck C, Kratz M, Torcasio A, et al. Comparison of optical coherence tomography, microcomputed tomography, and histology at a three-dimensionally imaged trabecular bone sample. J Biomed Opt. 2010;15:046019.

Kim YK, Kim YP. High-speed time-domain optical coherence tomography with an imaging speed of ten frames per second with 2000 A-scan. Opt Eng. 2010;49:055601.

Kim KH, Puoris'haag M, Maguluri GN, et al. Monitoring mouse retinal degeneration with high-resolution spectral-domain optical coherence tomography. J Vision. 2008;8:1701-11.

Kim J, Ahmad A, Boppart SA. Dual-coil magnetomotive optical coherence tomography for contrast enhancement in liquids. Opt Express. 2013;21:7139-47.

Kirsten L, Gaertner M, Schnabel C, Meissner S, Koch E. Four-dimensional imaging of murine subpleural alveoli using high-speed optical coherence tomography. J Biophotonics. 2013;6:148-52.

Kocaoglu OP, Uhlhorn SR, Hernandez E, et al. Simultaneous fundus imaging and optical coherence tomography of the mouse retina. Invest Ophthalmol Vis Sci. 2007;48:1283-9.

Kottig F, Cimalla P, Gartner M, Koch E. An advanced algorithm for dispersion encoded full range frequency domain optical coherence tomography. Opt Express. 2012;20:24925-48.

Larina IV, Larin KV, Justice MJ, Dickinson ME. Optical coherence tomography for live imaging of mammalian development. Curr Opin Genet Dev. 2011;21: 579-84.

Lee TM, Oldenburg AL, Sitafalwalla S, et al. Engineered microsphere contrast agents for optical coherence tomography. Opt Lett. 2003;28:1546-8.

Leitgeb R, Hitzenberger C, Fercher A. Performance of fourier domain vs. time domain optical coherence tomography. Opt Express. 2003a;11:889-94.

Leitgeb R, Schmetterer L, Drexler W, et al. Real-time assessment of retinal blood flow with ultrafast acquisition by color Doppler Fourier domain optical coherence tomography. Opt Express. 2003b;11:3116-21.

Li Q, Timmers AM, Hunter K, et al. Noninvasive imaging by optical coherence tomography to monitor retinal degeneration in the mouse. Invest Ophthalmol Vis Sci. 2001;42:2981-9.

Liu G, Chou L, Jia W, et al. Intensity-based modified Doppler variance algorithm: application to phase instable and phase stable optical coherence tomography systems. Opt Express. 2011;19:11429-40.

Liu G, Lin AJ, Tromberg BJ, Chen Z. A comparison of Doppler optical coherence tomography methods. Biomed Opt Express. 2012;3:2669-80.

Lorenser D, Yang X, Kirk RW, et al. Ultrathin sideviewing needle probe for optical coherence tomography. Opt Lett. 2011;36:3894-6.

Lu CW, Lee CK, Tsai MT, Wang YM, Yang CC. Measurement of the hemoglobin oxygen saturation level with spectroscopic spectral-domain optical coherence tomography. Opt Lett. 2008;33:416-8.

McLaughlin RA, Yang XJ, Quirk BC, et al. Static and dynamic imaging of alveoli using optical coherence tomography needle probes. J Appl Physiol. 2012;113:967-74.

Meissner S, Knels L, Koch E. Improved three-dimensional Fourier domain optical coherence tomography by index matching in alveolar structures. J Biomed Opt. 2009a; 14:064037.

Meissner S, Muller G, Walther J, Morawietz H, Koch E. In-vivo Fourier domain optical coherence tomography as a new tool for investigation of vasodynamics in the mouse model. J Biomed Opt. 2009b;14: 034027.

Meissner S, Knels L, Schnabel C, Koch T, Koch E. Threedimensional Fourier domain optical coherence tomography in vivo imaging of alveolar tissue in the intact thorax using the parietal pleura as a window. J Biomed Opt. 2010a;15:016030.

Meissner S, Tabuchi A, Mertens M, Kuebler WM, Koch E. Virtual four-dimensional imaging of lung parenchyma by optical coherence tomography in mice. J Biomed Opt. 2010b;15:036016.

Mertens M, Tabuchi A, Meissner S, et al. Alveolar dynamics in acute lung injury: heterogeneous distension rather than cyclic opening and collapse. Crit Care Med. 2009;37:2604-11.

Michelson AA. The relative motion of the earth and the lumniferous ether. Am J Sci. 1881;22:120-9.

Moayed AA, Hariri S, Hyun C, et al. Combined optical coherence tomography and electroretinography system for in vivo simultaneous morphological and functional imaging of the rodent retina. J Biomed Opt. 2010;15:040506.

Moayed AA, Hariri S, Choh V, Bizheva K. In vivo imaging of intrinsic optical signals in chicken retina with functional optical coherence tomography. Opt Lett. 2011;36:4575-7.

Morgner U, Drexler W, Kärtner FX, et al. Spectroscopic optical coherence tomography. Opt Lett. 2000;25:111-3.

Nagata A, Higashide T, Ohkubo S, Takeda H, Sugiyama $\mathrm{K}$. In vivo quantitative evaluation of the rat retinal nerve fiber layer with optical coherence tomography. Invest Ophthalmol Vis Sci. 2009;50:2809-15.

Namati E, Warger WC, Unglert CI, et al. Four-dimensional visualization of subpleural alveolar dynamics in vivo during uninterrupted mechanical ventilation of living swine. Biomed Opt Express. 2013;4:2492-506.

Oldenburg A, Toublan F, Suslick K, Wei A, Boppart S. Magnetomotive contrast for in vivo optical coherence tomography. Opt Express. 2005a;13:6597-614.

Oldenburg AL, Gunther JR, Boppart SA. Imaging magnetically labeled cells with magnetomotive optical coherence tomography. Opt Lett. 2005b;30: 747-9.

Oldenburg AL, Crecea V, Rinne SA, Boppart SA. Phaseresolved magnetomotive OCT for imaging nanomolar concentrations of magnetic nanoparticles in tissues. Opt Express. 2008;16:11525-39. 
Oldenburg AL, Hansen MN, Ralston TS, Wei A, Boppart SA. Imaging gold nanorods in excised human breast carcinoma by spectroscopic optical coherence tomography. J Mater Chem. 2009;19:6407-11.

Osiac E, Balseanu TA, Catalin B, et al. Optical coherence tomography as a promising imaging tool for brain investigations. Rom J Morphol Embryol. 2014;55:507-12.

Park BH, Saxer C, Srinivas SM, Nelson JS, de Boer JF. In vivo burn depth determination by high-speed fiberbased polarization sensitive optical coherence tomography. J Biomed Opt. 2001;6:474-9.

Povazay B, Bizheva K, Hermann B, et al. Enhanced visualization of choroidal vessels using ultrahigh resolution ophthalmic OCT at $1050 \mathrm{~nm}$. Opt Express. 2003;11:1980-6.

Povazay B, Hermann B, Unterhuber A, et al. Threedimensional optical coherence tomography at $1050 \mathrm{~nm}$ versus $800 \mathrm{~nm}$ in retinal pathologies: enhanced performance and choroidal penetration in cataract patients. J Biomed Opt. 2007;12:041211.

Povazay B, Hofer B, Torti C, et al. Impact of enhanced resolution, speed and penetration on three-dimensional retinal optical coherence tomography. Opt Express. 2009; 17:4134-50.

Quirk BC, McLaughlin RA, Pagnozzi AM, et al. Optofluidic needle probe integrating targeted delivery of fluid with optical coherence tomography imaging. Opt Lett. 2014;39:2888-91.

Robles FE, Wax A. Separating the scattering and absorption coefficients using the real and imaginary parts of the refractive index with low-coherence interferometry. Opt Lett. 2010;35:2843-5.

Robles F, Graf RN, Wax A. Dual window method for processing spectroscopic optical coherence tomography signals with simultaneously high spectral and temporal resolution. Opt Express. 2009;17:6799-812.

Robles FE, Wilson C, Grant G, Wax A. Molecular imaging true-colour spectroscopic optical coherence tomography. Nat Photonics. 2011;5:744-7.

Roggan A, Beuthan J, Schründer S, Müller G. Diagnostik und therapie mit dem laser. Physikalische Blätter. 1999;55:25-30.

Ruggeri M, Wehbe $\mathrm{H}$, Jiao $\mathrm{S}$, et al. In vivo threedimensional high-resolution imaging of rodent retina with spectral-domain optical coherence tomography. Invest Ophthalmol Vis Sci. 2007;48:1808-14.

Schmitt JM. Optical coherence tomography (OCT): a review. IEEE J Quantum Electron. 1999;5:1205-15.

Schmitt JM, Lee SL, Yung KM. An optical coherence microscope with enhanced resolving power in thick tissue. Opt Commun. 1997;142:203-7.

Schmoll T, Kolbitsch C, Leitgeb RA. In vivo functional retinal optical coherence tomography. J Biomed Opt. 2010;15:041513-041513.

Schnabel C, Gaertner M, Kirsten L, Meissner S, Koch E. Total liquid ventilation: a new approach to improve 3D OCT image quality of alveolar structures in lung tissue. Opt Express. 2013;21:31782-8.

Seeliger MW, Beck SC, Pereyra-Muñoz N, et al. In vivo confocal imaging of the retina in animal models using scanning laser ophthalmoscopy. Vision Res. 2005;45: 3512-9.

Srinivasan VJ, Ko TH, Wojtkowski M, et al. Noninvasive volumetric imaging and morphometry of the rodent retina with high-speed, ultrahigh-resolution optical coherence tomography. Invest Ophthalmol Vis Sci. 2006;47:5522-8.

Storen T, Royset A, Svaasand LO, Lindmo T. Measurement of dye diffusion in scattering tissue phantoms using dual-wavelength low-coherence interferometry. J Biomed Opt. 2006;11:014017.

Sun JJ, Guo SG, Wu L, et al. 3D In Vivo optical coherence tomography based on a low-voltage, large-scan-range 2D MEMS mirror. Opt Express. 2010;18:12065-75.

Szkulmowski M, Szkulmowska A, Bajraszewski T, Kowalczyk A, Wojtkowski M. Flow velocity estimation using joint spectral and time domain optical coherence tomography. Opt Express. 2008;16:6008-25.

Traub WA. Constant-dispersion grism spectrometer for channeled spectra. J Opt Soc Am A. 1990;7:1779-91.

Unterhuber A, Povazay B, Hermann B, et al. In vivo retinal optical coherence tomography at $1040 \mathrm{~nm}$ enhanced penetration into the choroid. Opt Express. 2005;13:3252-8

Vergnole S, Levesque D, Lamouche G. Experimental validation of an optimized signal processing method to handle non-linearity in swept-source optical coherence tomography. Opt Express. 2010;18:10446-61.

Walther J, Koch E. Transverse motion as a source of noise and reduced correlation of the Doppler phase shift in spectral domain OCT. Opt Express. 2009;17:19698-713.

Walther J, Koch E. Relation of joint spectral and time domain optical coherence tomography (jSTdOCT) and phase-resolved Doppler OCT. Opt Express. 2014;22:23129-46.

Walther J, Krüger A, Cuevas M, Koch E. Effects of axial, transverse, and oblique sample motion in FD OCT in systems with global or rolling shutter line detector. J Opt Soc Am A. 2008;25:2791-802.

Walther J, Mueller G, Morawietz H, Koch E. Signal power decrease due to fringe washout as an extension of the limited Doppler flow measurement range in spectral domain optical coherence tomography. J Biomed Opt. 2010;15:041511.

Wang L, Ho PP, Liu C, Zhang G, Alfano RR. Ballistic 2-D imaging through scattering walls using an ultrafast optical Kerr gate. Science. 1991;253:769-71.

Wang RK, Jacques SL, Ma Z, et al. Three dimensional optical angiography. Opt Express. 2007b;15:4083-97.

Wang J, Wang MR, Jiang H, et al. Detection of magnetic particles in live DBA/2J mouse eyes using magnetomotive optical coherence tomography. Eye Contact Lens. 2010b;36:346-51.

Wang L, Hofer B, Chen YP, et al. Highly reproducible swept-source, dispersion-encoded full-range biometry and imaging of the mouse eye. J Biomed Opt. 2010c; 15:046004.

Weber A, Hochmann S, Cimalla P, et al. Characterization of light lesion paradigms and optical coherence 
tomography as tools to study adult retina regeneration in zebrafish. PLoS One. 2013;8:e80483.

Wieser W, Biedermann BR, Klein T, Eigenwillig CM, Huber R. Multi-Megahertz OCT: High quality 3D imaging at 20 million A-scans and 4.5 GVoxels per second. Opt Express. 2010;18:14685-704.

Wojtkowski M, Srinivasan V, Ko T, et al. Ultrahighresolution, high-speed, Fourier domain optical coherence tomography and methods for dispersion compensation. Opt Express. 2004;12:2404-22.

Wolf E. Introduction to the theory of coherence and polarization of light. Wolf E, editors. Cambridge: Cambridge University Press; 2007.

Wolf-Schnurrbusch UEK, Ceklic L, Brinkmann CK, et al. Macular thickness measurements in healthy eyes using six different optical coherence tomography instruments. Invest Ophthalmol Vis Sci. 2009;50:3432-7.

$\mathrm{Xu}$ C, Ye J, Marks DL, Boppart SA. Near-infrared dyes as contrast-enhancing agents for spectroscopic optical coherence tomography. Opt Lett. 2004;29:1647-9.

Xu CY, Carney PS, Boppart SA. Wavelength-dependent scattering in spectroscopic optical coherence tomography. Opt Express. 2005;13:5450-62.

Yamanari M, Makita S, Yasuno Y. Polarization-sensitive swept-source optical coherence tomography with continuous source polarization modulation. Opt Express. 2008;16:5892-906.

Yang C, McGuckin LEL, Simon JD, et al. Spectral triangulation molecular contrast optical coherence tomography with indocyanine green as the contrast agent. Opt Lett. 2004a;29:2016-8.

Yang Y, Whiteman S, van Pittius DG, et al. Use of optical coherence tomography in delineating airways microstructure: comparison of OCT images to histopathological sections. Phys Med Biol. 2004b;49:1247-55.

Yi J, Radosevich AJ, Rogers JD, et al. Can OCT be sensitive to nanoscale structural alterations in biological tissue? Opt Express. 2013;21:9043-59.

Yi J, Radosevich AJ, Stypula-Cyrus Y, et al. Spatially resolved optical and ultrastructural properties of colorectal and pancreatic field carcinogenesis observed by inverse spectroscopic optical coherence tomography. J Biomed Opt. 2014;19:036013-036013.

Yin JC, Liu GJ, Zhang J, et al. In vivo early detection of smoke-induced airway injury using three-dimensional swept-source optical coherence tomography. J Biomed Opt. 2009;14:060503.

Yoo KM, Alfano RR. Determination of the scattering and absorption lengths from the temporal profile of a backscattered pulse. Opt Lett. 1990;15:276-8.

Yun SH, Tearney G, de Boer J, Bouma B. Motion artifacts in optical coherence tomography with frequencydomain ranging. Opt Express. 2004;12:2977-98.

Zhang Y, Cense B, Rha J, et al. High-speed volumetric imaging of cone photoreceptors with adaptive optics spectral-domain optical coherence tomography. Opt Express. 2006;14:4380-94.

Zhou X, Xie J, Shen M, et al. Biometric measurement of the mouse eye using optical coherence tomography with focal plane advancement. Vision Res. 2008;48:1137-43.

\section{Intravital Imaging in Small Animals}

Brown EB, Campbell RB, Tsuzuki Y, Xu L, Carmeliet P, Fukumura D, et al. In vivo measurement of gene expression, angiogenesis and physiological function in tumors using multiphoton laser scanning microscopy. Nat Med. 2001;7(7):864-8.

Burford JL, Villanueva K, Lam L, Riquier-Brison A, Hackl MJ, Pippin J, et al. Intravital imaging of podocyte calcium in glomerular injury and disease. J Clin Invest. 2014;124(5):2050-8.

Chevre R, Gonzalez-Granado JM, Megens RT, Sreeramkumar V, Silvestre-Roig C, Molina-Sanchez $\mathrm{P}$, et al. High-resolution imaging of intravascular atherogenic inflammation in live mice. Circ Res. 2014;114(5):770-9.

Chung K, Deisseroth K. CLARITY for mapping the nervous system. Nat Methods. 2013;10(6):508-13.

Denk W, Strickler JH, Webb WW. Two-photon laser scanning fluorescence microscopy. Science. 1990;248(4951): 73-6.

Dietzel S, Pircher J, Nekolla AK, Gull M, Brandli AW, Pohl U, et al. Label-free determination of hemodynamic parameters in the microcirculaton with third harmonic generation microscopy. PLoS One. 2014; 9(6):e99615.

Douma K, Prinzen L, Slaaf DW, Reutelingsperger CP, Biessen EA, Hackeng TM, et al. Nanoparticles for optical molecular imaging of atherosclerosis. Small. 2009;5(5):544-57.

Eichhoff G, Garaschuk O. Two-photon imaging of neural networks in a mouse model of Alzheimer's disease. Cold Spring Harb Protoc. 2011;2011(10):1206-16.

Endrich B, Hammersen F, Messmer K. Capillary ultrastructure and microcirculatory function of malignant tumors. Adv Exp Med Biol. 1988;242:205-14.

Fan GY, Fujisaki H, Miyawaki A, Tsay RK, Tsien RY, Ellisman MH. Video-rate scanning two-photon excitation fluorescence microscopy and ratio imaging with cameleons. Biophys J. 1999;76(5):2412-20.

Furie B, Furie BC. Thrombus formation in a living mouse. Pathophysiol Haemost Thromb. 2006;35(1-2):1-4.

Genina EA, Bashkatov AN, Kolesnikova EA, Basko MV, Terentyuk GS, Tuchin VV. Optical coherence tomography monitoring of enhanced skin optical clearing in rats in vivo. J Biomed Opt. 2014;19(2):21109.

Gobel W, Kampa BM, Helmchen F. Imaging cellular network dynamics in three dimensions using fast 3D laser scanning. Nat Methods. 2007;4(1):73-9.

Grewe BF, Langer D, Kasper H, Kampa BM, Helmchen F. High-speed in vivo calcium imaging reveals neuronal network activity with near-millisecond precision. Nat Methods. 2010;7(5):399-405.

Helmchen F, Denk W. Deep tissue two-photon microscopy. Nat Methods. 2005;2(12):932-40.

Julius Cohnheim (1839-1884) experimental pathologist. JAMA :J Am Med Assoc. 1968;206(7):1561-2.

Kamoun WS, Chae SS, Lacorre DA, Tyrrell JA, Mitre M, Gillissen MA, et al. Simultaneous measurement of 
$\mathrm{RBC}$ velocity, flux, hematocrit and shear rate in vascular networks. Nat Methods. 2010;7(8):655-60.

Kapsokalyvas D, Schiffers P, Maij N, Suylen D, Hackeng $\mathrm{T}$, Van Zandvoort M, et al. Imaging evidence for endothelin ETA/ETB receptor heterodimers in isolated rat mesenteric resistance arteries. Life Sci. 2014; 111(1-2):36-41.

Kiyooka T, Hiramatsu O, Shigeto F, Nakamoto $\mathrm{H}$, Tachibana H, Yada T, et al. Direct observation of epicardial coronary capillary hemodynamics during reactive hyperemia and during adenosine administration by intravital video microscopy. Am J Physiol Heart Circ Physiol. 2005;288(3):H1437-43.

Kubes P, Payne D, Woodman RC. Molecular mechanisms of leukocyte recruitment in postischemic liver microcirculation. Am J Physiol Gastrointest Liver Physiol. 2002;283(1):G139-47.

Lammermann T, Afonso PV, Angermann BR, Wang JM, Kastenmuller W, Parent CA, et al. Neutrophil swarms require LTB4 and integrins at sites of cell death in vivo. Nature. 2013;498(7454):371-5.

Levene MJ, Dombeck DA, Kasischke KA, Molloy RP, Webb WW. In vivo multiphoton microscopy of deep brain tissue. J Neurophysiol. 2004;91(4):1908-12.

Li X, Yu W. Deep tissue microscopic imaging of the kidney with a gradient-index lens system. Opt Commun. 2008;281(7):1833-40.

Li W, Goldstein DR, Kreisel D. Intravital 2-photon imaging, leukocyte trafficking, and the beating heart. Trends Cardiovasc Med. 2013b;23(8):287-93.

Livet J, Weissman TA, Kang H, Draft RW, Lu J, Bennis $\mathrm{RA}$, et al. Transgenic strategies for combinatorial expression of fluorescent proteins in the nervous system. Nature. 2007;450(7166):56-62.

Mahou P, Zimmerley M, Loulier K, Matho KS, Labroille G, Morin X, et al. Multicolor two-photon tissue imaging by wavelength mixing. Nat Methods. 2012;9(8): 815-8.

Massberg S, Gawaz M, Gruner S, Schulte V, Konrad I, Zohlnhofer D, et al. A crucial role of glycoprotein VI for platelet recruitment to the injured arterial wall in vivo. J Exp Med. 2003;197(1):41-9.

Megens RT, Kemmerich K, Pyta J, Weber C, Soehnlein O. Intravital imaging of phagocyte recruitment. Thromb Haemost. 2011;105(5):802-10.

Menger MD, Laschke MW, Vollmar B. Viewing the microcirculation through the window: some twenty years experience with the hamster dorsal skinfold chamber. Eur Surg Res Europaische chirurgische Forschung Recherches chirurgicales europeennes. 2002;34(1-2):83-91.

Mittmann W, Wallace DJ, Czubayko U, Herb JT, Schaefer AT, Looger LL, et al. Two-photon calcium imaging of evoked activity from L5 somatosensory neurons in vivo. Nat Neurosci. 2011;14(8):1089-93.

Mizuno R, Kamioka Y, Kabashima K, Imajo M, Sumiyama $\mathrm{K}$, Nakasho E, et al. In vivo imaging reveals PKA regulation of ERK activity during neutrophil recruitment to inflamed intestines. J Exp Med. 2014;211(6): 1123-36.
Nielsen T, Fricke M, Hellweg D, Andresen P. High efficiency beam splitter for multifocal multiphoton microscopy. J Microsc. 2001;201(Pt 3):368-76.

Niesner R, Andresen V, Neumann J, Spiecker H, Gunzer M. The power of single and multibeam two-photon microscopy for high-resolution and high-speed deep tissue and intravital imaging. Biophys J. 2007; 93(7):2519-29.

Progatzky F, Dallman MJ, Lo CC. From seeing to believing: labelling strategies for in vivo cell-tracking experiments. Interface Focus. 2013;3(3):20130001.

Rademakers T, Douma K, Hackeng TM, Post MJ, Sluimer JC, Daemen MJ, et al. Plaque-associated vasa vasorum in aged apolipoprotein E-deficient mice exhibit proatherogenic functional features in vivo. Arterioscler Thromb Vasc Biol. 2013;33(2):249-56.

Rothstein EC, Carroll S, Combs CA, Jobsis PD, Balaban RS. Skeletal muscle NAD(P)H two-photon fluorescence microscopy in vivo: topology and optical inner filters. Biophys J. 2005;88(3):2165-76.

Schepers AG, Snippert HJ, Stange DE, van den Born M, van Es JH, van de Wetering M, et al. Lineage tracing reveals Lgr5+ stem cell activity in mouse intestinal adenomas. Science. 2012;337(6095):730-5.

Shaner NC, Patterson GH, Davidson MW. Advances in fluorescent protein technology. J Cell Sci. 2007;120(Pt 24):4247-60.

Thorlacius H, Lindbom L, Raud J. Cytokine-induced leukocyte rolling in mouse cremaster muscle arterioles in P-selectin dependent. Am J Physiol. 1997;272(4 Pt 2):H1725-9.

van Zandvoort M, Engels W, Douma K, Beckers L, Oude Egbrink M, Daemen M, et al. Two-photon microscopy for imaging of the (atherosclerotic) vascular wall: a proof of concept study. J Vasc Res. 2004;41(1): 54-63.

Victora GD, Schwickert TA, Fooksman DR, Kamphorst AO, Meyer-Hermann M, Dustin ML, et al. Germinal center dynamics revealed by multiphoton microscopy with a photoactivatable fluorescent reporter. Cell. 2010;143(4):592-605.

Weber K, Thomaschewski M, Warlich M, Volz T, Cornils $\mathrm{K}$, Niebuhr B, et al. RGB marking facilitates multicolor clonal cell tracking. Nat Med. 2011;17(4):504-9.

Woodfin A, Voisin MB, Beyrau M, Colom B, Caille D, Diapouli FM, et al. The junctional adhesion molecule JAM-C regulates polarized transendothelial migration of neutrophils in vivo. Nat Immunol. 2011;12(8): 761-9.

\section{Optoacoustic Imaging}

Allen TJ, Beard PC. Pulsed near-infrared laser diode excitation system for biomedical photoacoustic imaging. Opt Lett. 2006b;31:3462-4.

Amiot L, Xu SP, Liang S, Pan LY, Zhao JXJ. Near-infrared fluorescent materials for sensing of biological targets. Sensors. 2008;8:3082-105. 
Beziere N, von Schacky C, Kosanke Y, et al. Optoacoustic imaging and staging of inflammation in a murine model of arthritis. Arth Rheumatol. 2014;66:2071-8.

Buehler A, Herzog E, Razansky D, Ntziachristos V. Video rate optoacoustic tomography of mouse kidney perfusion. Opt Lett. 2010b;35:2475-7.

De La Z, Zavaleta C, Keren S, Vaithilingam S, Bodapati S, Liu Z, Levi J, Smith BR, Ma TJ, Oralkan O, et al. Carbon nanotubes as photoacoustic molecular imaging agents in living mice. Nat Nanotech. 2008;3: 557-62.

Deán-Ben XL, Razansky D. Adding fifth dimension to optoacoustic imaging: volumetric time-resolved spectrallyenriched tomography. Light Sci Appl. 2014;3:e137.

Duck FA. Physical properties of tissues: a comprehensive reference book. San Diego: Academic; 1990.

Herzog E, Taruttis A, Beziere N, Lutich A, Razansky D, Ntziachristos V. Optical imaging of cancer heterogeneity with multispectral optoacoustic tomography. Radiology. 2012;263:461-8.

Hu S, Maslov K, Wang LV. Second generation opticalresolution photoacoustic microscopy with improved sensitivity and speed. Opt Lett. 2011;36:1134-6.

Hudson SV, Huang JS, Yin W, Albeituni S, Rush J, Khanal A, Yan J, Ceresa BP, Frieboes HB, McNally LR. Targeted noninvasive imaging of EGFR-expressing orthotopic pancreatic cancer using multispectral optoacoustic tomography. Cancer Res. 2014;74: 6271-9.

Ida T, Kawaguchi Y, Kawauchi S, et al. Real-time photoacoustic imaging system for burn diagnosis. J Biomed Opt. 2014;19:086013.

Jansen $\mathrm{K}, \mathrm{Wu} \mathrm{M}$, van der Steen AFW, van Soest G. Photoacoustic imaging of human coronary atherosclerosis in two spectral bands. Photoacoustics. 2014; 2(1):12-20.

Kostli KP, Frenz M, Weber HP, Paltauf G, SchmidtKloiber H. Optoacoustic infrared spectroscopy of soft tissue. J Appl Phys. 2000;88:1632-7.

Kruger RA, Liu P, Fang YR, Appledorn CR. Photoacoustic ultrasound (PAUS)--reconstruction tomography. Med Phys. 1995;22:1605-9.

Krumholz A, Vanvickle-Chavez SJ, Yao J, et al. Photoacoustic microscopy of tyrosinase reporter gene in vivo. J Biomed Opt. 2011;16:80503.

Laufer J, Delpy D, Elwell C, Beard P. Quantitative spatially resolved measurement of tissue chromophore concentrations using photoacoustic spectroscopy: application to the measurement of blood oxygenation and haemoglobin concentration. Phys Med Biol. 2007;52(1):141-68.

Laufer J, Jathoul A, Johnson P, Zhang E, Lythgoe M, Pedley RB, Pule M, Beard P. In vivo photoacoustic imaging of tyrosinase expressing tumours in mice. Proc SPIE. 2012;822.

Lozano N, Al-Ahmady ZS, Beziere NS, Ntziachristos V, Kostarelos K. Monoclonal antibody-targeted PEGylated liposome-ICG encapsulating doxorubicin as a potential theranostic agent. Int $\mathrm{J}$ Pharm. 2014;482:2-10. doi:10.1016/j.ijpharm.2014.10.045.
Lutzweiler C, Razansky D. Optoacoustic imaging and tomography: reconstruction approaches and outstanding challenges in image performance and quantification. Sensors. 2013;13:7345-84.

Mehrmohammadi M, Yoon SJ, Yeager D, Emelianov SY. Photoacoustic imaging for cancer detection and staging. Curr Mol Imag. 2013;2:89-105.

Nasiriavanaki M, Xia J, Wan H, Bauer AQ, Culver JP, Wang LV. High-resolution photoacoustic tomography of resting-state functional connectivity in the mouse brain. Proc Natl Acad Sci U S A. 2013;111:21-6.

Ntziachristos V, Razansky D. Molecular imaging by means of multispectral opto-acoustic tomography (MSOT). Chem Rev. 2010;110:2783-94.

Ntziachristos V, Ripoll J, Wang LV, Weissleder R. Looking and listening to light: the evolution of whole-body photonic imaging. Nat Biotechnol. 2005;23:313-20.

Razansky D. Multispectral optoacoustic tomography volumetric color hearing in real time. IEEE J Sel Top Quant Electron. 2012;18:1234-43.

Razansky D, Distel M, Vinegoni C, Ma R, Perrimon N, Köster RW, Ntziachristos V. Multi-spectral optoacoustic tomography of deep-seated fluorescent proteins invivo. Nat Photon. 2009;3:412-7.

Razansky D, Kellnberger S, Ntziachristos V. Near-field radio-frequency thermoacoustic tomography with impulse excitation. Med Phys. 2010;37:4602-7.

Razansky D, Buehler A, Ntziachristos V. Volumetric realtime multispectral optoacoustic tomography of biomarkers. Nat Prot. 2011;6:1121-9.

Razansky D, Deliolanis N, Vinegoni C, Ntziachristos V. Deep tissue optical and optoacoustic molecular imaging technologies for small animal research and drug discovery. Curr Pharm Biotechnol. 2012;13: 504-22.

Rosencwaig A. Photoacoustic spectroscopy of biological materials. Science. 1973;181:657-8.

Sethuraman S, Amirian JH, Litovsky SH, Smalling RW, Emelianov SY. Spectroscopic intravascular photoacoustic imaging to differentiate atherosclerotic plaques. Opt Express. 2008;16:3362-7.

Song L, Kim C, Maslov K, Shung KK, Wang LHV. Highspeed dynamic 3D photoacoustic imaging of sentinel lymph node in a murine model using an ultrasound array. Med Phys. 2009;36:3724-9.

Tainter CS, Bell AG. Selenium and the photophone. Nature. 1880;22:500-3.

Taruttis A, Herzog E, Razansky D, et al. Real-time imaging of cardiovascular dynamics and circulating gold nanorods with multispectral optoacoustic tomography. Opt Express. 2010;18:19592-602.

Taruttis A, Morscher S, Burton NC, Razansky D, Ntziachristos V. Fast multispectral optoacoustic tomography (MSOT) for dynamic imaging of pharmacokinetics and biodistribution in multiple organs. PLoS One. 2012; 7:e30491.

Tong L, Wei QS, Wei A, Cheng JX. Gold nanorods as contrast agents for biological imaging: optical properties, surface conjugation and photothermal effects. Photochem Photobiol. 2009;85:21-32. 
VonnemannJ,BeziereN,BöttcherC, etal.Polyglycerolsulfate functionalized gold nanorods as optoacoustic signal nanoamplifiers for in vivo bioimaging of rheumatoid arthritis. Theranostics. 2014;4:629-41.

AU28 Wang LV, editor. Photoacoustic imaging and spectroscopy. CRC Press; 2009.

Wang $\mathrm{LV}, \mathrm{Hu} \mathrm{S}$. Photoacoustic tomography: in vivo imaging from organelles to organs. Science. 2012;335: 1458-62.

Wang XD, Pang YJ, Ku G, Xie XY, Stoica G, Wang LHV. Noninvasive laser-induced photoacoustic tomography for structural and functional in vivo imaging of the brain. Nat Biotechnol. 2003;21:803-6.

Wang Y, Maslov K, Zhang Y, Hu S, Yang LM, Xia YN, Liu JA, Wang LHV. Fiber-laser-based photoacoustic microscopy and melanoma cell detection. J Biomed Opt. 2011;16:011014.

Yang JM, Favazza C, Chen RM, Yao JJ, Cai X, Maslov K, Zhou QF, Shung KK, Wang LHV. Simultaneous functional photoacoustic and ultrasonic endoscopy of internal organs in vivo. Nat Med. 2012;18:1297-302.

Yao J, Wang LV. Sensitivity of photoacoustic microscopy. Photoacoustics. 2014;2:87-101.

\section{Optical Probes}

Alander JT, Kaartinen I, Laakso A, Pätilä T, Spillmann T, Tuchin VV, Venermo M, Välisuo P. A review of indocyanine green fluorescent imaging in surgery. Int J Biomed Imaging. 2012;2012:940585.

Azar S, Intes X. Translational multimodality optical imaging. Boston: Artech House; 2008.

Barnett EM, Zhang X, Maxwell D, Chang Q, PiwnicaWorms D. Single-cell imaging of retinal ganglion cell apoptosis with a cell-penetrating, activatable peptide probe in an in vivo glaucoma model. Proc Natl Acad Sci U S A. 2009;106:9391-6.

Becker A, Hessenius C, Licha K, Ebert B, Sukowski U, Semmler W, Wiedenmann B, Grötzinger C. Receptortargeted optical imaging of tumors with near-infrared fluorescent ligands. Nat Biotechnol. 2001;19:327-31.

Bentolila LA, Ebenstein Y, Weiss S. Quantum dots for in vivo small-animal imaging. J Nucl Med. 2009;50:493-6.

Boens N, Leen V, Dehaen W. Fluorescent indicators based on BODIPY. Chem Soc Rev. 2012;41:1130-72.

Bremer C, Tung $\mathrm{CH}$, Weissleder R. In vivo molecular target assessment of matrix metalloproteinase inhibition. Nat Med. 2001b;7:743-8.

Fischer T, Gemeinhardt I, Wagner S, Stieglitz DV, Schnorr J, Hermann KG, Ebert B, Petzelt D, Macdonald R, Licha K, Schirner M, Krenn V, Kamradt T, Taupitz M. Assessment of unspecific near-infrared dyes in laser-induced fluorescence imaging of experimental arthritis. Acad Radiol. 2006;13:4-13.

Foster AE, Kwon S, Ke S, Lu A, Eldin K, Sevick-Muraca $\mathrm{E}$, Rooney CM. In vivo fluorescent optical imaging of cytotoxic T lymphocyte migration using IRDye $800 \mathrm{CW}$ near-infrared dye. Appl Opt. 2008;47:5944-52.
Hermanson GT. Bioconjugate techniques. San Diego: Academic; 1996.

Hilderbrand SA, Weissleder R. Near-infrared fluorescence: application to in vivo molecular imaging. Curr Opin Chem Biol. 2010;14:71-9.

Holzinger M, Le Goff A, Cosnier S. Nanomaterials for biosensing applications: a review. Front Chem. 2014;2:63.

Kisin-Finfer E, Ferber S, Blau R, Satchi-Fainaro R, Shabat D. Synthesis and evaluation of new NIRfluorescent probes for cathepsin B: ICT versus FRET as a turn-ON mode-of-action. Bioorg Med Chem Lett. 2014;24:2453-8.

Klohs J, Steinbrink J, Bourayou R, Mueller S, Cordell R, Licha K, Schirner M, Dirnagl U, Lindauer U, Wunder A. Near-infrared fluorescence imaging with fluorescently labeled albumin: a novel method for noninvasive optical imaging of blood-brain barrier impairment after focal cerebral ischemia in mice. J Neurosci Methods. 2009;180(1):126-32.

Lee SB, Hassan M, Fisher R, Chertov O, Chernomordik V, Kramer-Marek G, Gandjbakhche A, Capala J. Affibody molecules for in vivo characterization of HER2-positive tumors by near-infrared imaging. Clin Cancer Res. 2008;14:3840-9.

Lee H, Akers W, Bhushan K, Bloch S, Sudlow G, Tang R. Near-infrared $\mathrm{pH}$-activatable fluorescent probes for imaging primary and metastatic breast tumors. Bioconjug Chem. 2011;22:777-84.

Licha K, Resch-Genger U. Probes for optical imaging: new developments. Drug Discovery Today: Technol. 2011;8:e87-94.

Licha K, Resch-Genger U. Fluorescent reporters and optical probes. In: Brahme A. (Editor in Chief) Comprehensive biomedical physics, vol. 4. Amsterdam: Elsevier; 2014. p. 85-109.

Licha K, Schirner M, Henry G. Optical agents. In: Schwaiger M, editor Handbook of experimental pharmacology, vol 185, part 2. Heidelberg: Springer, p. 203-22.

Luo S, Zhang E, Su Y, Cheng T, Shi C. A review of NIR dyes in cancer targeting and imaging. Biomaterials. 2011b;32:7127-38.

Mathejczyk JE, Pauli J, Dullin C, Resch-Genger U, Alves F, Napp J. High-sensitivity detection of breast tumors in vivo by use of a $\mathrm{pH}$-sensitive near-infrared fluorescence probe. J Biomed Opt. 2012;17:076028.

Mier W, Beijer B, Graham K, Hull WE. Fluorescent somatostatin receptor probes for the intraoperative detection of tumor tissue with long-wavelength visible light. Bioorg Med Chem. 2002;10:2543-52.

Moon WK, Lin Y, O'Loughlin T, Tang Y, Kim DE, Weissleder R, Tung $\mathrm{CH}$. Enhanced tumor detection using a folate receptor-targeted near-infrared fluorochrome conjugate. Bioconjug Chem. 2003;14: 539-45.

Pauli J, Vag T, Haag R, Spieles M, Wenzel M, Kaiser WA, Resch-Genger U, Hilger I. An in vitro characterization study of new near infrared dyes for molecular imaging. Eur J Med Chem. 2009b;44:3496-503. 
Pauli J, Licha K, Berkemeyer J, Grabolle M, Spieles M, Wegner N, Welker P, Resch-Genger U. New fluorescent labels with tunable hydrophilicity for the rational design of bright optical probes for molecular imaging. Bioconjug Chem. 2013;24:1174-85.

Quadir QA, Radowski MR, Kratz F, Licha K, Hauff P, Haag R. Dendritic multishell architectures for drug and dye transport. J Control Release. 2008;132: 289-94.

Rasmussen JC, Tan IC, Marshall MV, Fife CE, SevickMuraca EM. Lymphatic imaging in humans with nearinfrared fluorescence. Curr Opin Biotechnol. 2009;20:74-82.

Resch-Genger U, Grabolle M, Cavaliere-Jaricot S, Nitschke R, Nann T. Quantum dots versus organic dyes as fluorescent labels. Nat Methods. 2008;9: 763-75.

Richards G, Soubrane G, Yanuzzi L. Fluorescein and ICG angiography. Germany: Thieme; 1998.

Selvam S, Kundu K, Templeman KL, Murthy N, Garcia AJ. Minimally invasive, longitudinal monitoring of biomaterial-associated inflammation by fluorescence imaging. Biomaterials. 2011;32:7785-92.

Stefflova K, Chen J, Zheng G. Using molecular beacons for cancer imaging and treatment. Front Biosci. 2007;12:4709-21.

Ulrich G, Ziessel R, Harriman A. The chemistry of fluorescent bodipy dyes: versatility unsurpassed. Angew Chem Int Ed. 2008;47:1184-201.

Umezawa K, Citterio D, Suzuki K. New trends in nearinfrared fluorophores for bioimaging. Anal Sci. 2014;30:327-49.

Wall A, Persigehl T, Hauff P, Licha K, Schirner M, Müller S, von Wallbrunn A, Matuszewski L, Heindel W, Bremer C. Differentiation of angiogenic burden in human cancer xenografts using a perfusion-type optical contrast agent (SIDAG). Breast Cancer Res. 2008;10:R23.

Weissleder R, Tung CH, Mahmood U, Bogdanov Jr A. In vivo imaging of tumors with protease-activated nearinfrared fluorescent probes. Nat Biotechnol. 1999;17: 375-8. 


\section{Author Queries}

Chapter No.: 16

0002794118

\begin{tabular}{|c|c|c|}
\hline Queries & Details Required & Author's Response \\
\hline AU1 & Please provide email address for the corresponding authors. & \\
\hline AU2 & $\begin{array}{l}\text { We placed the affiliation detail of Editor "Fabian Kiessling" in place for the "Ingrid } \\
\text { Hilger and Marc A.M.J. van Zandvoort". Please provide us the missing affiliation } \\
\text { details. }\end{array}$ & \\
\hline AU3 & Please confirm the abstract. [metadata content] & \\
\hline AU4 & Please provide other items if appropriate. & \\
\hline AU5 & $\begin{array}{l}\text { Figures have been renumbered to maintain sequential order of citation in text. Please } \\
\text { check. }\end{array}$ & \\
\hline AU6 & Please provide closing parenthesis. & \\
\hline AU7 & Please provide other items if appropriate. & \\
\hline AU8 & Please check if edit to sentence starting "Several porphyrin derivatives..." is okay. & \\
\hline AU9 & Please provide opening parenthesis. & \\
\hline AU10 & Please check sentence starting "Injection of D-luciferin..." for clarity. & \\
\hline AU11 & Please check if edit to sentence starting "There are several opportunities..." is okay. & \\
\hline AU12 & Please confirm if inserted citation for Fig. 16.13 is okay. & \\
\hline AU13 & $\begin{array}{l}\text { Leitgeb et al. (2000), Xu et al. (2008), Adler et al. (2008), Tucker-Schwartz et al. } \\
\text { (2014), Yim et al. (2014), Kuranov et al. (2011a )and Kuranov et al. (2011b) are cited } \\
\text { in the text but not provided in the reference list. Please check. }\end{array}$ & \\
\hline AU14 & Please check if edit to sentence starting "An example of a..." is okay. & \\
\hline AU15 & Please check sentence starting "All images were..." for completeness. & \\
\hline AU16 & $\begin{array}{l}\text { Please check latter part of sentence starting "One of the most elegant..." for } \\
\text { completeness. }\end{array}$ & \\
\hline AU17 & Please check sentence starting "Both are related..." for completeness. & \\
\hline AU18 & $\begin{array}{l}\text { Please check sentence starting "The relation between concentrations ..." for } \\
\text { completeness. }\end{array}$ & \\
\hline AU19 & Please provide appropriate chapter cross reference for "Chap. 21.2 .5 " here. & \\
\hline AU20 & Please provide appropriate chapter cross reference for "Chap. 21.3" here. & \\
\hline AU21 & Please provide appropriate chapter cross reference for "Chap. 21.3.3" here. & \\
\hline AU22 & Please provide caption for part figures "e" in Fig. 16.44. & \\
\hline AU23 & Please provide appropriate chapter cross reference for "Chap. 21.2" here. & \\
\hline AU24 & $\begin{array}{l}\text { Please provide citation for Faber and van Leeuwen (2009), Licha et al. (2008), } \\
\text { Resch-Genger et al. (2008), and Ulrich et al. (2008) in text. }\end{array}$ & \\
\hline AU25 & $\begin{array}{l}\text { Please provide volume number for Osterman and Schutz-Geschwender (2007) and } \\
\text { Cammidge and Gopee (2002). }\end{array}$ & \\
\hline AU26 & Please provide page range for Saha et al. (2011) and Laufer et al. (2012). & \\
\hline AU27 & Please provide journal title and volume number for Bukowska et al. (2010). & \\
\hline AU28 & Please provide publisher location for Wang (2009). & \\
\hline
\end{tabular}

